\title{
On relationships between two linear subspaces and two orthogonal projectors
}

https://doi.org/110.1515/spma-2019-0013

Received June 16, 2019; accepted September 22, 2019

Abstract: Sum and intersection of linear subspaces in a vector space over a field are fundamental operations in linear algebra. The purpose of this survey paper is to give a comprehensive approach to the sums and intersections of two linear subspaces and their orthogonal complements in the finite-dimensional complex vector space. We shall establish a variety of closed-form formulas for representing the direct sum decompositions of the $m$-dimensional complex column vector space $\mathbb{C}^{m}$ with respect to a pair of given linear subspaces $\mathcal{M}$ and $\mathcal{N}$ and their operations, and use them to derive a huge amount of decomposition identities for matrix expressions composed by a pair of orthogonal projectors onto the linear subspaces. As applications, we give matrix representation for the orthogonal projectors onto the intersections of a pair of linear subspaces using various matrix decomposition identities and Moore-Penrose inverses; necessary and sufficient conditions for two linear subspaces to be in generic position; characterization of the commutativity of a pair of orthogonal projectors; necessary and sufficient conditions for equalities and inequalities for a pair of subspaces to hold; equalities and inequalities for norms of a pair of orthogonal projectors and their operations; as well as a collection of characterizations of EP-matrix.

Keywords: Linear subspace, orthogonal complement, sum, intersection, orthogonal projector, matrix decomposition, Moore-Penrose inverse, generic position, commutativity, norm, EP matrix

MSC: 15A03, 15A09, 15A24, 15A27, 15B57, 47A15, 47H05

\section{Introduction}

Throughout this paper, we adopt the following notation:

$\mathbb{R}^{m \times n}, \mathbb{C}^{m \times n}$, and $\mathbb{C}_{\mathrm{H}}^{m}$ stand for the collections of all $m \times n$ real, complex matrices, and all $m \times m$ complex Hermitian matrices, respectively;

$\mathbb{C}^{m}=\mathbb{C}^{m \times 1}\left(\mathbb{R}^{m}=\mathbb{R}^{m \times 1}\right)$ denotes the $n$-dimensional complex (real) vector space;

$r(A), \mathscr{R}(A)$, and $\mathscr{N}(A)$ stand for the rank, the range (column space), and the kernel (null space) of a matrix $A \in \mathbb{C}^{m \times n}$, respectively;

$I_{m}$ denotes the identity matrix of order $m$;

$[A, B]$ stands for the columnwise partitioned matrix;

$A \succ 0, A \succcurlyeq 0, A \prec 0$, and $A \preccurlyeq 0$ mean that $A$ is positive definite, positive semi-definite, negative definite, and negative semi-definite, respectively;

two $A, B \in \mathbb{C}_{\mathrm{H}}^{m}$ are said to satisfy the inequalities $A \succ B, A \succcurlyeq B, A \prec B$, and $A \preccurlyeq B$ in the Löwner partial ordering if $A-B$ is positive definite, positive semi-definite, negative definite, and negative semi-definite, respectively;

both $i_{+}(A)$ and $i_{-}(A)$, called the partial inertia of $A \in \mathbb{C}_{\mathrm{H}}^{m}$, are defined to be the numbers of the positive and negative eigenvalues of $A$ counted with multiplicities, respectively, where $r(A)=i_{+}(A)+i_{-}(A)$;

^Corresponding Author: Yongge Tian: Shanghai Business School, Shanghai, China \& Central University of Finance and Economics, Beijing, China, E-mail: yongge.tian@gmail.com 
the parallel sum of two positive semi-definite matrices $A, B \in \mathbb{C}_{\mathrm{H}}^{m}$ is defined to be $A: B=A(A+B)^{\dagger} B$; the group inverse of $A \in \mathbb{C}^{m \times m}$ with $r\left(A^{2}\right)=r(A)$, denoted by $A^{\#}$, is defined to be the unique solution $X$ satisfying the three matrix equations $A X A=A, X A X=X$ and $A X=X A$;

the orthogonal projector onto $\mathcal{M}$ is a Hermitian idempotent matrix $X$, denoted by $P_{\mathcal{M}}$, that satisfies range $(X)=\mathcal{M}$; the collection of all orthogonal projectors of order $m$ is denoted by $\mathbb{C}_{\mathrm{OP}}^{m}$;

a complex matrix $U \in \mathbb{C}^{m \times m}$ is unitary if $U^{*} U=U U^{*}=I_{m}$; a $Q \in \mathbb{R}^{m \times m}$ is call an orthogonal matrix if $Q^{T} Q=Q Q^{T}=I_{m}$.

We next introduce definitions of generalized inverses of a matrix. The Moore-Penrose inverse of a given matrix $A \in \mathbb{C}^{m \times n}$, denoted by $A^{\dagger}$, is defined to be the unique matrix $X \in \mathbb{C}^{n \times m}$ satisfying the four Penrose equations

$$
\text { (i) } A X A=A \text {, (ii) } X A X=X \text {, (iii) }(A X)^{*}=A X \text {, (iv) }(X A)^{*}=X A \text {. }
$$

In addition, a matrix $X$ is called an $\{i, \ldots, j\}$-inverse of $A$, denoted by $A^{(i, \ldots, j)}$, if it satisfies the $i$ th, $\ldots, j$ th equations. The collection of all $\{i, \ldots, j\}$-inverses of $A$ is denoted by $\left\{A^{(i, \ldots, j)}\right\}$. There are all 15 types of $\{i, \ldots, j\}$-inverse under (1.1), but the eight commonly-used generalized inverses of $A$ induced from the first equation are given by $A^{\dagger}, A^{(1,3,4)}, A^{(1,2,4)}, A^{(1,2,3)}, A^{(1,4)}, A^{(1,3)}, A^{(1,2)}$, and $A^{(1)}$. We also denote by $P_{A}=A A^{\dagger}$, $E_{A}=I_{m}-A A^{\dagger}$, and $F_{A}=I_{n}-A^{\dagger} A$ the three orthogonal projectors induced by $A$. Note from the definitions of generalized inverses of a matrix that they are in fact defined to be (common) solutions of some matrix equations. Thus analytical expressions of generalized inverses of matrix can be written as certain matrix-valued functions with one or more variable matrices. In fact, analytical formulas of generalized inverses of matrices and their functions are important issues and tools in matrix analysis, see e.g., [32, 48, 120].

Linear subspaces and orthogonal projectors onto linear subspaces are simplest and most generic concepts in linear algebra. These time-honored concepts are the parts of cornerstones in current mathematics, and are regarded as the very starting point of many advanced research topics in mathematics. Let $\mathcal{M}$ be a linear subspace of the $n$-dimensional complex column vector space $\mathbb{C}^{m}$. The orthogonal projector onto $\mathcal{M}$ is a Hermitian idempotent matrix $X=X^{*}=X^{2}$, denoted by $P_{\mathcal{M}}$, that satisfies range $(X)=\mathcal{M}$. Orthogonal projectors have many simple and nice properties from the viewpoint of matrices and operator theory, which can be characterized by many equations and facts; see, e.g., $[75,146]$. On the other hand, orthogonal projectors onto linear subspaces are classic objects of study in linear vector spaces, and many properties of linear subspaces can be characterized by orthogonal projectors and their operations. Much attention has been paid to algebraic operations of two or more orthogonal projectors and their performance in various algebraic structures, and many results and facts on this topic can be found in the literature; see e.g., $[1-4,6,9,10,12,16,17,20-24,27-30$, 3335, 38, 41-43, 49-52, 63, 64, 69, 71, 73, 94-97, 99, 102, 110, 113, 115, 117, 119, 121, 131, 138, 145, 148, 149, 153]. As a summary, the aim of this paper is to provide a most detailed description on twists of two linear subspaces and two orthogonal projectors within the scope of linear algebra. The main content o this paper includes the following compilation of subjects:

(I) establish simultaneous direct sum decomposition identities for two linear subspaces;

(II) give many formulas for pure algebraic operations of two orthogonal projectors;

(III) reformulate the CS decomposition for the pair of orthogonal projectors, and use it to derive many identities for the Moore-Penrose inverses of two orthogonal projectors and their algebraic operations;

(IV) give four groups of matrix representation for the four orthogonal projectors onto the intersection subspaces, and present a group of identifying conditions for two linear subspaces to be in generic position;

(V) give a collection of equivalent statements for two orthogonal projectors to be commutative;

(VI) give a collection of equivalent statements for subspace equalities and inequalities to hold;

(VII) give equalities and inequalities for representing the F-norms of two orthogonal projectors and their operations;

(VIII) give collections of equivalent statements for a square matrix to be EP.

A huge amount of formulas, equalities, inequalities, and facts are presented in 8 sections below covering the above topics, which will update our total understanding to linear subspaces and orthogonal projectors from all aspects. Most of the contents collected in this paper are so elementary and informative that we believe that they can definitely serve as beautiful and unreplaceable constructive materials for future textbooks in linear algebra and matrix theory. 


\section{Fundamentals on a pair of linear subspaces}

In this section, we first present some fundamental facts on finite-dimensional vector space. Let $K$ be a field (such as the real or complex number field), and $V$ a vector space over $K$, and let $\mathcal{N}$ and $\mathcal{N}$ be two linear subspaces in $V$. Recall in linear algebra that the sum and the intersection of $\mathcal{N}$ and $\mathcal{N}$ are defined to be

$$
\begin{aligned}
& \mathcal{M}+\mathcal{N}=\{x+y \mid x \in \mathcal{M} \text { and } y \in \mathcal{N}\}, \\
& \mathcal{M} \cap \mathcal{N}=\{x \mid \text { both } x \in \mathcal{M} \text { and } x \in \mathcal{N}\},
\end{aligned}
$$

respectively, both of which are linear subspaces in $V$ as well. In particular,

$$
\mathcal{W}=\mathcal{M} \oplus \mathcal{N} \Longleftrightarrow \mathcal{W}=\mathcal{M}+\mathcal{N} \text { and } \mathcal{M} \cap \mathcal{N}=\{0\},
$$

where $\mathcal{M} \oplus \mathcal{N}$ is called the direct sum of $\mathcal{M}$ and $\mathcal{N}$. Recall that the standard inner product of any two complex (real) column vectors $x, y$ in $\mathbb{C}^{m}\left(\mathbb{R}^{m}\right)$ is defined by

$$
\langle x, y\rangle=x^{*} y \quad\left(\langle x, y\rangle=x^{T} y\right) .
$$

Given the inner product, the orthogonality of two vectors $x$ and $y$, denoted by $x \perp y$, is defined by the requirement

$$
\langle x, y\rangle=0,
$$

and two linear subspaces $\mathcal{M}$ and $\mathcal{N}$ of $\mathbb{C}^{m}$ are said to be orthogonal, denoted by

$$
\mathcal{M} \perp \mathcal{N}
$$

if and only if $x \perp y$ for all $x \in \mathcal{M}$ and $y \in \mathcal{N}$. If $\mathcal{M} \perp \mathcal{N}$, it must be the case that $\mathcal{M}$ and $\mathcal{N}$ are disjoint: $\mathcal{M} \cap \mathcal{N}=\{0\}$. Given a subspace $\mathcal{N}$ of $\mathbb{C}^{m}$, the orthogonal complement of $\mathcal{M}$ of $\mathbb{C}^{m}$ is defined to be

$$
\mathcal{M}^{\perp}=\left\{y \in \mathbb{C}^{m} \mid\langle x, y\rangle=0 \text { for all } x \in \mathcal{M}\right\} .
$$

Recall that the dimension of a finite-dimensional vector space (linear subspace), denoted by $\operatorname{dim}(\cdot)$, is the number of independent vectors required to span the space (linear subspace). A best-known basic formula linking the dimensions of the sum and the intersection of two subspaces $\mathcal{M}_{1}$ and $\mathcal{M}_{2}$ in finite-dimensional vector space $V$ is

$$
\operatorname{dim}\left(\mathcal{M}_{1}+\mathcal{M}_{2}\right)+\operatorname{dim}\left(\mathcal{M}_{1} \cap \mathcal{M}_{2}\right)=\operatorname{dim}\left(\mathcal{M}_{1}\right)+\operatorname{dim}\left(\mathcal{M}_{2}\right),
$$

see e.g., $[105,126]$. This formula shows that the dimensions the sum and the intersection of two linear subspaces are related, thus it can be used to describe relationship between two linear subspaces, for instance,

$$
\operatorname{dim}\left(\mathcal{M}_{1}+\mathcal{M}_{2}\right)=\operatorname{dim}\left(\mathcal{M}_{1}\right)+\operatorname{dim}\left(\mathcal{M}_{2}\right) \Longleftrightarrow \mathcal{M}_{1} \cap \mathcal{M}_{2}=\{0\}
$$

One of the examples of the linear spaces is the range (column space) of a given matrix. Now let $\mathcal{M}_{1}=\mathscr{R}\left(A_{1}\right)$ and $\mathcal{M}_{2}=\mathscr{R}\left(A_{2}\right)$ be two linear subspaces spanned by the column vectors of two matrices $A_{1} \in \mathbb{C}^{m \times n_{1}}$ and $A_{2} \in \mathbb{C}^{m \times n_{2}}$, respectively. Then the dimension formula in (2.8) can alternatively be expressed as

$$
r\left[A_{1}, A_{2}\right]+\operatorname{dim}\left[\mathscr{R}\left(A_{1}\right) \cap \mathscr{R}\left(A_{2}\right)\right]=r\left(A_{1}\right)+r\left(A_{2}\right) .
$$

These basic results and facts can be found in almost all textbooks of linear algebra, and play a central role in the theory of finite-dimensional vector space. An extension of (2.8) with symmetric pattern to a family of linear subspaces $\mathcal{M}_{1}, \mathcal{M}_{2}, \ldots, \mathcal{M}_{k}$ in $\mathbb{C}^{m}$ has recently been established by the present author in [139] as follows

$$
(k-1) \operatorname{dim}\left(\mathcal{M}_{1}+\cdots+\mathcal{M}_{k}\right)+\operatorname{dim}\left(\widehat{\mathcal{M}}_{1} \cap \cdots \cap \widehat{\mathcal{M}}_{k}\right)=\operatorname{dim}\left(\widehat{\mathcal{M}}_{1}\right)+\cdots+\operatorname{dim}\left(\widehat{\mathcal{M}}_{k}\right),
$$

where $\widehat{\mathcal{M}}_{i}=\mathcal{M}_{1}+\cdots+\mathcal{M}_{i-1}+\mathcal{M}_{i+1}+\cdots+\mathcal{M}_{k}, i=1,2, \ldots, k$.

In addition, we need to use in the sequel the following known results and facts; see e.g., [81, 93, 105, 126]. 
Lemma 2.1. Let $\mathcal{M}$ and $\mathcal{N}$ be two linear subspaces in $\mathbb{C}^{m}$. Then the following results hold.

(a) $\mathcal{M}$ and its orthogonal complement obey the following rules

$$
\left(\mathcal{M}^{\perp}\right)^{\perp}=\mathcal{M}, \mathcal{M} \oplus \mathcal{M}^{\perp}=\mathbb{C}^{m}, \operatorname{dim} \mathcal{M}+\operatorname{dim} \mathcal{M}^{\perp}=m .
$$

In particular,

$$
\left(\mathbb{C}^{m}\right)^{\perp}=\{0\} \text { and }\{0\}^{\perp}=\mathbb{C}^{m}
$$

(b) The following results hold

$$
\begin{aligned}
& (\mathcal{M}+\mathcal{N})^{\perp}=\mathcal{M}^{\perp} \cap \mathcal{N}^{\perp},(\mathcal{M} \cap \mathcal{N})^{\perp}=\mathcal{M}^{\perp}+\mathcal{N}^{\perp}, \\
& \mathcal{M} \subseteq \mathcal{N} \Leftrightarrow \mathcal{M}^{\perp} \supseteq \mathcal{N}^{\perp} .
\end{aligned}
$$

Lemma 2.2. Let $\mathcal{M}_{1}, \mathcal{M}_{2}$, and $\mathcal{M}_{3}$ be three linear subspaces of $\mathbb{C}^{m}$. Then the following results hold.

(a) $\mathcal{M}_{1}, \mathcal{M}_{2}$, and $\mathcal{M}_{3}$ satisfy the distributive inequalities

$$
\begin{aligned}
& \mathcal{M}_{1} \cap\left(\mathcal{M}_{2}+\mathcal{M}_{3}\right) \supseteq\left(\mathcal{M}_{1} \cap \mathcal{M}_{2}\right)+\left(\mathcal{M}_{1} \cap \mathcal{M}_{3}\right), \\
& \mathcal{M}_{1}+\left(\mathcal{M}_{2} \cap \mathcal{M}_{3}\right) \subseteq\left(\mathcal{M}_{1}+\mathcal{M}_{2}\right) \cap\left(\mathcal{M}_{1}+\mathcal{M}_{3}\right),
\end{aligned}
$$

or equivalently, there exist two subspaces $\mathcal{M}^{\prime}$ and $\mathcal{M}^{\prime \prime}$, called residual subspaces, such that

$$
\begin{aligned}
& \mathcal{M}_{1} \cap\left(\mathcal{M}_{2}+\mathcal{M}_{3}\right)=\left[\left(\mathcal{M}_{1} \cap \mathcal{M}_{2}\right)+\left(\mathcal{M}_{1} \cap \mathcal{M}_{3}\right)\right] \oplus \mathcal{M}^{\prime}, \\
& {\left[\mathcal{M}_{1}+\left(\mathcal{M}_{2} \cap \mathcal{M}_{3}\right)\right] \oplus \mathcal{M}^{\prime \prime}=\left(\mathcal{M}_{1}+\mathcal{M}_{2}\right) \cap\left(\mathcal{M}_{1}+\mathcal{M}_{3}\right) .}
\end{aligned}
$$

(b) The following modular identities hold

$$
\begin{aligned}
& \mathcal{M}_{1} \supseteq \mathcal{M}_{2} \Leftrightarrow \mathcal{M}_{1} \cap\left(\mathcal{M}_{2}+\mathcal{M}_{3}\right)=\mathcal{M}_{2}+\left(\mathcal{M}_{1} \cap \mathcal{M}_{3}\right), \\
& \text { both } \mathcal{M}_{1} \supseteq \mathcal{M}_{2} \text { and } \mathcal{M}_{1} \supseteq \mathcal{M}_{3} \Leftrightarrow \mathcal{M}_{1} \cap\left(\mathcal{M}_{2}+\mathcal{M}_{3}\right)=\mathcal{M}_{2}+\mathcal{M}_{3} .
\end{aligned}
$$

(c) The following orthomodular law holds

$$
\mathcal{M}_{1} \supseteq \mathcal{M}_{2} \Leftrightarrow \mathcal{M}_{1}=\left(\mathcal{M}_{1} \cap \mathcal{M}_{2}^{\frac{1}{2}}\right) \oplus \mathcal{M}_{2} \Leftrightarrow \mathcal{M}_{1}^{\perp}=\left(\mathcal{M}_{1}^{\perp}+\mathcal{M}_{2}\right) \cap \mathcal{M}_{2}^{\perp} .
$$

(d) The following symmetricity of commutativity holds

$$
\mathcal{M}_{1}=\left(\mathcal{M}_{1} \cap \mathcal{M}_{2}\right) \oplus\left(\mathcal{M}_{1} \cap \mathcal{M}_{2}^{\frac{1}{2}}\right) \Leftrightarrow \mathcal{M}_{2}=\left(\mathcal{M}_{1} \cap \mathcal{M}_{2}\right) \oplus\left(\mathcal{M}_{1}^{\perp} \cap \mathcal{M}_{2}\right) .
$$

Assume that two linear subspaces in a vector space are given. One of the basic research problems in a vector space theory is concerned with the decompositions of the vector space with respect to the pair of subspaces. This kind of problem was first proposed and approached in [80]; see also [42, 43, 66, 71, 93]. Now assume that $\mathcal{M}$ and $\mathcal{N}$ are two linear subspaces of $\mathbb{C}^{m}$. Then there are four intersections

$$
\mathcal{M} \cap \mathcal{N}, \mathcal{M} \cap \mathcal{N}^{\perp}, \mathcal{M}^{\perp} \cap \mathcal{N}, \mathcal{M}^{\perp} \cap \mathcal{N}^{\perp}
$$

induced from $\mathcal{M}, \mathcal{N}, \mathcal{M}^{\perp}$, and $\mathcal{N}^{\perp}$. By (2.8), a set of dimension formulas associated with the above four intersections are given below.

Lemma 2.3. Let $\mathcal{M}$ and $\mathcal{N}$ be two linear subspaces in $\mathbb{C}^{m}$. Then

$$
\begin{aligned}
& \operatorname{dim}(\mathcal{M}+\mathcal{N})=\operatorname{dim}(\mathcal{N})+\operatorname{dim}(\mathcal{N})-\operatorname{dim}(\mathcal{M} \cap \mathcal{N}) \\
& \operatorname{dim}\left(\mathcal{M}+\mathcal{N}^{\perp}\right)=\operatorname{dim}(\mathcal{N})+\operatorname{dim}\left(\mathcal{N}^{\perp}\right)-\operatorname{dim}\left(\mathcal{M} \cap \mathcal{N}^{\perp}\right) \\
& \operatorname{dim}\left(\mathcal{M}^{\perp}+\mathcal{N}\right)=\operatorname{dim}\left(\mathcal{M}^{\perp}\right)+\operatorname{dim}(\mathcal{N})-\operatorname{dim}\left(\mathcal{M}^{\perp} \cap \mathcal{N}\right) \\
& \operatorname{dim}\left(\mathcal{M}^{\perp}+\mathcal{N}^{\perp}\right)=\operatorname{dim}\left(\mathcal{M}^{\perp}\right)+\operatorname{dim}\left(\mathcal{N}^{\perp}\right)-\operatorname{dim}\left(\mathcal{M}^{\perp} \cap \mathcal{N}^{\perp}\right)
\end{aligned}
$$


Furthermore, some well-known decompositions of $\mathcal{M}, \mathcal{N}$, and $\mathbb{C}^{m}$ with respect to (2.24) are formulated follows.

Lemma 2.4. Let $\mathcal{M}$ and $\mathcal{N}$ be two linear subspaces in $\mathbb{C}^{m}$. Then

$$
\begin{aligned}
& \mathcal{M}=\mathcal{M} \cap \mathbb{C}^{m}=\mathcal{M} \cap\left(\mathcal{N} \oplus \mathcal{N}^{\perp}\right)=(\mathcal{M} \cap \mathcal{N}) \oplus\left(\mathcal{M} \cap \mathcal{N}^{\perp}\right) \oplus \text { rest }, \\
& \mathcal{N}=\mathbb{C}^{m} \cap \mathcal{N}=\left(\mathcal{M} \oplus \mathcal{M}^{\perp}\right) \cap \mathcal{N}=(\mathcal{M} \cap \mathcal{N}) \oplus\left(\mathcal{M}^{\perp} \cap \mathcal{N}\right) \oplus \text { rest }, \\
& \mathcal{M}+\mathcal{N}=(\mathcal{M} \cap \mathcal{N}) \oplus\left(\mathcal{M} \cap \mathcal{N}^{\perp}\right) \oplus\left(\mathcal{M}^{\perp} \cap \mathcal{N}\right) \oplus \text { rest, } \\
& \mathbb{C}^{m}=(\mathcal{M} \cap \mathcal{N}) \oplus\left(\mathcal{M} \cap \mathcal{N}^{\perp}\right) \oplus\left(\mathcal{M}^{\perp} \cap \mathcal{N}\right) \oplus\left(\mathcal{M}^{\perp} \cap \mathcal{N}^{\perp}\right) \oplus \text { rest. }
\end{aligned}
$$

The whole space $\mathbb{C}^{m}$ can be decomposed into the following four forms

$$
\begin{aligned}
& \mathbb{C}^{m}=(\mathcal{M}+\mathcal{N})^{\perp} \oplus(\mathcal{M}+\mathcal{N})=\left(\mathcal{M}^{\perp} \cap \mathcal{N}^{\perp}\right) \oplus\left(\mathcal{M}^{\perp} \cap \mathcal{N}^{\perp}\right)^{\perp}=\left(\mathcal{M}^{\perp} \cap \mathcal{N}^{\perp}\right) \oplus(\mathcal{M}+\mathcal{N}), \\
& \mathbb{C}^{m}=\left(\mathcal{M}^{\perp}+\mathcal{N}\right)^{\perp} \oplus\left(\mathcal{M}^{\perp}+\mathcal{N}\right)=\left(\mathcal{M} \cap \mathcal{N}^{\perp}\right) \oplus\left(\mathcal{M} \cap \mathcal{N}^{\perp}\right)^{\perp}=\left(\mathcal{M} \cap \mathcal{N}^{\perp}\right) \oplus\left(\mathcal{M}^{\perp}+\mathcal{N}\right) \text {, } \\
& \mathbb{C}^{m}=\left(\mathcal{M}+\mathcal{N}^{\perp}\right)^{\perp} \oplus\left(\mathcal{M}+\mathcal{N}^{\perp}\right)=\left(\mathcal{M}^{\perp} \cap \mathcal{N}\right) \oplus\left(\mathcal{M}^{\perp} \cap \mathcal{N}\right)^{\perp}=\left(\mathcal{M}^{\perp} \cap \mathcal{N}\right) \oplus\left(\mathcal{M}+\mathcal{N}^{\perp}\right) \text {, } \\
& \mathbb{C}^{m}=\left(\mathcal{M}^{\perp}+\mathcal{N}^{\perp}\right)^{\perp} \oplus\left(\mathcal{M}^{\perp}+\mathcal{N}^{\perp}\right)=(\mathcal{M} \cap \mathcal{N}) \oplus(\mathcal{M} \cap \mathcal{N})^{\perp}=(\mathcal{M} \cap \mathcal{N}) \oplus\left(\mathcal{M}^{\perp}+\mathcal{N}^{\perp}\right) \text {. }
\end{aligned}
$$

The following direct sum decomposition hold

$$
\begin{aligned}
& \mathcal{M}=\mathcal{M} \cap \mathbb{C}^{m}=\mathcal{M} \cap\left[(\mathcal{M} \cap \mathcal{N}) \oplus(\mathcal{M} \cap \mathcal{N})^{\perp}\right]=(\mathcal{M} \cap \mathcal{N}) \oplus\left[\mathcal{M} \cap(\mathcal{M} \cap \mathcal{N})^{\perp}\right], \\
& \mathcal{M}=\mathcal{M} \cap \mathbb{C}^{m}=\mathcal{M} \cap\left[\left(\mathcal{M} \cap \mathcal{N}^{\perp}\right) \oplus\left(\mathcal{M} \cap \mathcal{N}^{\perp}\right)^{\perp}\right]=\left(\mathcal{M} \cap \mathcal{N}^{\perp}\right) \oplus\left[\mathcal{M} \cap\left(\mathcal{M} \cap \mathcal{N}^{\perp}\right)^{\perp}\right], \\
& \mathcal{N}=\mathcal{N} \cap \mathbb{C}^{m}=\mathcal{N} \cap\left[(\mathcal{M} \cap \mathcal{N}) \oplus(\mathcal{M} \cap \mathcal{N})^{\perp}\right]=(\mathcal{M} \cap \mathcal{N}) \oplus\left[\mathcal{N} \cap(\mathcal{M} \cap \mathcal{N})^{\perp}\right] \text {, } \\
& \mathcal{N}=\mathcal{N} \cap \mathbb{C}^{m}=\mathcal{N} \cap\left[\left(\mathcal{M}^{\perp} \cap \mathcal{N}\right) \oplus\left(\mathcal{M}^{\perp} \cap \mathcal{N}\right)^{\perp}\right]=\left(\mathcal{M}^{\perp} \cap \mathcal{N}\right) \oplus\left[\mathcal{N} \cap\left(\mathcal{M}^{\perp} \cap \mathcal{N}\right)^{\perp}\right], \\
& \mathcal{M}^{\perp}=\mathcal{M}^{\perp} \cap \mathbb{C}^{m}=\mathcal{M}^{\perp} \cap\left[\left(\mathcal{M}^{\perp} \cap \mathcal{N}\right) \oplus\left(\mathcal{K}^{\perp} \cap \mathcal{N}\right)^{\perp}\right]=\left(\mathcal{M}^{\perp} \cap \mathcal{N}\right) \oplus\left[\mathcal{M}^{\perp} \cap\left(\mathcal{K}^{\perp} \cap \mathcal{N}\right)^{\perp}\right] \text {, } \\
& \mathcal{M}^{\perp}=\mathcal{M}^{\perp} \cap \mathbb{C}^{m}=\mathcal{M}^{\perp} \cap\left[\left(\mathcal{M}^{\perp} \cap \mathcal{N}^{\perp}\right) \oplus\left(\mathcal{M}^{\perp} \cap \mathcal{N}^{\perp}\right)^{\perp}\right]=\left(\mathcal{M}^{\perp} \cap \mathcal{N}^{\perp}\right) \oplus\left[\mathcal{M}^{\perp} \cap\left(\mathcal{M}^{\perp} \cap \mathcal{N}^{\perp}\right)^{\perp}\right] \text {, } \\
& \mathcal{N}^{\perp}=\mathcal{N}^{\perp} \cap \mathbb{C}^{m}=\mathcal{N}^{\perp} \cap\left[\left(\mathcal{M} \cap \mathcal{N}^{\perp}\right) \oplus(\mathcal{M} \cap \mathcal{N})^{\perp}\right]=\left(\mathcal{M} \cap \mathcal{N}^{\perp}\right) \oplus\left[\mathcal{N}^{\perp} \cap\left(\mathcal{M} \cap \mathcal{N}^{\perp}\right)^{\perp}\right] \\
& \mathcal{N}^{\perp}=\mathcal{N}^{\perp} \cap \mathbb{C}^{m}=\mathcal{N}^{\perp} \cap\left[\left(\mathcal{M}^{\perp} \cap \mathcal{N}^{\perp}\right) \oplus\left(\mathcal{M}^{\perp} \cap \mathcal{N}^{\perp}\right)^{\perp}\right]=\left(\mathcal{M}^{\perp} \cap \mathcal{N}^{\perp}\right) \oplus\left[\mathcal{N}^{\perp} \cap\left(\mathcal{M}^{\perp} \cap \mathcal{N}^{\perp}\right)^{\perp}\right] .
\end{aligned}
$$

The decompositions in (2.25)-(2.28) and the analytical expressions of the rest components were approached, e.g., in [42, 43, 66, 71, 80, 93]. Eqs. (2.29)-(2.32) follow from (2.12)-(2.14). Eqs. (2.33)-(2.40) follow from (2.12) and (2.20).

In the remaining of this section, we give a variety of novel formulas and facts on (2.25)-(2.28) and their variations. For convenience of representation, we define a binary operation of subspaces $\mathcal{M}_{1}$ and $\mathcal{M}_{2}$ as follows

$$
\mathcal{M}_{1} \circ \mathcal{M}_{2} \triangleq \mathcal{M}_{1} \cap\left(\mathcal{M}_{1} \cap \mathcal{M}_{2}^{\perp}\right)^{\perp}=\mathcal{M}_{1} \cap\left(\mathcal{M}_{1}^{\perp}+\mathcal{M}_{2}\right) .
$$

In term of this notation, we have the following eight expressions:

$$
\begin{aligned}
& \mathcal{M} \circ \mathcal{N}=\mathcal{M} \cap\left(\mathcal{M} \cap \mathcal{N}^{\perp}\right)^{\perp}=\mathcal{M} \cap\left(\mathcal{M}^{\perp}+\mathcal{N}\right), \\
& \mathcal{N} \circ \mathcal{M}=\mathcal{N} \cap\left(\mathcal{M}^{\perp} \cap \mathcal{N}\right)^{\perp}=\mathcal{N} \cap(\mathcal{M}+\mathcal{N})^{\perp}, \\
& \mathcal{M} \circ \mathcal{N}^{\perp}=\mathcal{M} \cap(\mathcal{M} \cap \mathcal{N})^{\perp}=\mathcal{M} \cap\left(\mathcal{M}^{\perp}+\mathcal{N}^{\perp}\right), \\
& \mathcal{N} \perp \circ \mathcal{M}=\mathcal{N}^{\perp} \cap\left(\mathcal{M}^{\perp} \cap \mathcal{N}^{\perp}\right)^{\perp}=\mathcal{N}^{\perp} \cap(\mathcal{M}+\mathcal{N}), \\
& \mathcal{M}^{\perp} \circ \mathcal{N}=\mathcal{M}^{\perp} \cap\left(\mathcal{M}^{\perp} \cap \mathcal{N}^{\perp}\right)^{\perp}=\mathcal{M}^{\perp} \cap(\mathcal{M}+\mathcal{N}), \\
& \mathcal{N} \circ \mathcal{M}^{\perp}=\mathcal{N} \cap(\mathcal{M} \cap \mathcal{N})^{\perp}=\mathcal{N} \cap\left(\mathcal{M}^{\perp}+\mathcal{N} \mathcal{N}^{\perp}\right), \\
& \mathcal{M}^{\perp} \circ \mathcal{N}^{\perp}=\mathcal{M}^{\perp} \cap\left(\mathcal{M}^{\perp} \cap \mathcal{N}\right)^{\perp}=\mathcal{M}^{\perp} \cap\left(\mathcal{M}+\mathcal{N}^{\perp}\right), \\
& \mathcal{N}^{\perp} \circ \mathcal{M}^{\perp}=\mathcal{N}^{\perp} \cap\left(\mathcal{M} \cap \mathcal{N}^{\perp}\right)^{\perp}=\mathcal{N}^{\perp} \cap\left(\mathcal{M}^{\perp}+\mathcal{N}\right) .
\end{aligned}
$$

Correspondingly,

$$
(\mathcal{M} \circ \mathcal{N})^{\perp}=\mathcal{M}^{\perp} \oplus\left(\mathcal{M} \cap \mathcal{N}^{\perp}\right), \quad(\mathcal{N} \circ \mathcal{M})^{\perp}=\mathcal{N}^{\perp} \oplus\left(\mathcal{M}^{\perp} \cap \mathcal{N}\right),
$$




$$
\begin{aligned}
& \left(\mathcal{M} \circ \mathcal{N}^{\perp}\right)^{\perp}=\mathcal{M}^{\perp} \cap(\mathcal{M} \cap \mathcal{N}), \quad\left(\mathcal{N}^{\perp} \circ \mathcal{M}\right)^{\perp}=\mathcal{N} \oplus\left(\mathcal{M}^{\perp} \cap \mathcal{N}^{\perp}\right), \\
& \left(\mathcal{M}^{\perp} \circ \mathcal{N}\right)^{\perp}=\mathcal{M} \oplus\left(\mathcal{M}^{\perp} \cap \mathcal{N}^{\perp}\right),\left(\mathcal{N} \circ \mathcal{M}^{\perp}\right)^{\perp}=\mathcal{N}^{\perp} \oplus(\mathcal{M} \cap \mathcal{N}) \text {, } \\
& \left(\mathcal{M}^{\perp} \circ \mathcal{N}^{\perp}\right)^{\perp}=\mathcal{M} \oplus\left(\mathcal{M}^{\perp} \cap \mathcal{N}\right),\left(\mathcal{N}^{\perp} \circ \mathcal{M}^{\perp}\right)^{\perp}=\mathcal{N} \oplus\left(\mathcal{M} \cap \mathcal{N}^{\perp}\right) \text {. }
\end{aligned}
$$

Substituting (2.41)-(2.48) into (2.33)-(2.40), we obtain a group of simultaneous direct sum decomposition identities as follows.

Theorem 2.5. Let $\mathcal{M}$ and $\mathcal{N}$ be two linear subspaces of $\mathbb{C}^{m}$. Then the following results hold.

(a) $\mathcal{M}, \mathcal{N}, \mathcal{M}^{\perp}$, and $\mathcal{N}^{\perp}$ can simultaneously be decomposed as

$$
\begin{aligned}
& \mathcal{M}=(\mathcal{M} \cap \mathcal{N}) \oplus\left(\mathcal{M} \circ \mathcal{N}^{\perp}\right)=\left(\mathcal{M} \cap \mathcal{N}^{\perp}\right) \oplus(\mathcal{M} \circ \mathcal{N}), \\
& \mathcal{N}=(\mathcal{M} \cap \mathcal{N}) \oplus\left(\mathcal{N} \circ \mathcal{M}^{\perp}\right)=\left(\mathcal{M}^{\perp} \cap \mathcal{N}\right) \oplus(\mathcal{N} \circ \mathcal{M}), \\
& \mathcal{M}^{\perp}=\left(\mathcal{M}^{\perp} \cap \mathcal{N}\right) \oplus\left(\mathcal{M}^{\perp} \circ \mathcal{N}^{\perp}\right)=\left(\mathcal{M}^{\perp} \cap \mathcal{N}^{\perp}\right) \oplus\left(\mathcal{M}^{\perp} \circ \mathcal{N}\right), \\
& \mathcal{N}^{\perp}=\left(\mathcal{M} \cap \mathcal{N}^{\perp}\right) \oplus\left(\mathcal{N}^{\perp} \circ \mathcal{M}^{\perp}\right)=\left(\mathcal{M}^{\perp} \cap \mathcal{N}^{\perp}\right) \oplus\left(\mathcal{N}^{\perp} \circ \mathcal{M}\right) .
\end{aligned}
$$

(b) The following disjoint equalities hold

$$
\begin{array}{ll}
\left(\mathcal{N} \circ \mathcal{N}^{\perp}\right) \cap\left(\mathcal{N} \circ \mathcal{M}^{\perp}\right)=\{0\}, & (\mathcal{M} \circ \mathcal{N}) \cap\left(\mathcal{N}^{\perp} \circ \mathcal{M}^{\perp}\right)=\{0\}, \\
\left(\mathcal{M}^{\perp} \circ \mathcal{N}^{\perp}\right) \cap(\mathcal{N} \circ \mathcal{M})=\{0\}, & \left(\mathcal{M}^{\perp} \circ \mathcal{N}\right) \cap\left(\mathcal{N}^{\perp} \circ \mathcal{N}\right)=\{0\} .
\end{array}
$$

(c) The following direct sum decomposition identities hold

$$
\begin{aligned}
& \mathcal{M}+\mathcal{N}=(\mathcal{M} \cap \mathcal{N}) \oplus\left(\mathcal{M} \circ \mathcal{N}^{\perp}\right) \oplus\left(\mathcal{N} \circ \mathcal{M}^{\perp}\right), \\
& \mathcal{M}+\mathcal{N}^{\perp}=\left(\mathcal{M} \cap \mathcal{N}^{\perp}\right) \oplus(\mathcal{M} \circ \mathcal{N}) \oplus\left(\mathcal{N}^{\perp} \circ \mathcal{M}^{\perp}\right), \\
& \mathcal{M}^{\perp}+\mathcal{N}=\left(\mathcal{M}^{\perp} \cap \mathcal{N}\right) \oplus\left(\mathcal{M}^{\perp} \circ \mathcal{N}^{\perp}\right) \oplus(\mathcal{N} \circ \mathcal{M}), \\
& \mathcal{M}^{\perp}+\mathcal{N}^{\perp}=\left(\mathcal{M}^{\perp} \cap \mathcal{N}^{\perp}\right) \oplus\left(\mathcal{M}^{\perp} \circ \mathcal{N}\right) \oplus\left(\mathcal{N}^{\perp} \circ \mathcal{M}\right), \\
& \mathbb{C}^{m}=\left(\mathcal{M} \cap \mathcal{N}^{\perp}\right) \oplus\left(\mathcal{M}^{\perp} \cap \mathcal{N}^{\perp}\right) \oplus(\mathcal{M} \circ \mathcal{N}) \oplus\left(\mathcal{M}^{\perp} \circ \mathcal{N}\right) \text {, } \\
& \mathbb{C}^{m}=\left(\mathcal{M}^{\perp} \cap \mathcal{N}\right) \oplus\left(\mathcal{M}^{\perp} \cap \mathcal{N}^{\perp}\right) \oplus(\mathcal{N} \circ \mathcal{M}) \oplus\left(\mathcal{N}^{\perp} \circ \mathcal{M}\right), \\
& \mathbb{C}^{m}=(\mathcal{M} \cap \mathcal{N}) \oplus\left(\mathcal{M}^{\perp} \cap \mathcal{N}^{\perp}\right) \oplus\left(\mathcal{M} \circ \mathcal{N}^{\perp}\right) \oplus\left(\mathcal{N} \circ \mathcal{M}^{\perp}\right) \text {, } \\
& \mathbb{C}^{m}=\left(\mathcal{M} \cap \mathcal{N}^{\perp}\right) \oplus\left(\mathcal{M}^{\perp} \cap \mathcal{N}\right) \oplus(\mathcal{M} \circ \mathcal{N}) \oplus\left(\mathcal{N}^{\perp} \circ \mathcal{M}^{\perp}\right) \text {, } \\
& \mathbb{C}^{m}=\left(\mathcal{M}^{\perp} \cap \mathcal{N}\right) \oplus\left(\mathcal{M} \cap \mathcal{N}^{\perp}\right) \oplus\left(\mathcal{M}^{\perp} \circ \mathcal{N}^{\perp}\right) \oplus(\mathcal{N} \circ \mathcal{M}) \text {, } \\
& \mathbb{C}^{m}=(\mathcal{M} \cap \mathcal{N}) \oplus\left(\mathcal{M}^{\perp} \cap \mathcal{N}^{\perp}\right) \oplus\left(\mathcal{M}^{\perp} \circ \mathcal{N}\right) \oplus\left(\mathcal{N}^{\perp} \circ \mathcal{M}\right) .
\end{aligned}
$$

(d) [77] The following direct sum decomposition identities hold

$$
\begin{aligned}
\mathbb{C}^{m} & =(\mathcal{N} \circ \mathcal{N}) \oplus(\mathcal{N} \circ \mathcal{M})^{\perp}=\left(\mathcal{M}^{\perp} \circ \mathcal{N}\right) \oplus\left(\mathcal{N} \circ \mathcal{M}^{\perp}\right)^{\perp} \\
& =\left(\mathcal{M} \circ \mathcal{N}^{\perp}\right) \oplus\left(\mathcal{N}^{\perp} \circ \mathcal{M}\right)^{\perp}=\left(\mathcal{M}^{\perp} \circ \mathcal{N}^{\perp}\right) \oplus\left(\mathcal{N}^{\perp} \circ \mathcal{M}^{\perp}\right)^{\perp} .
\end{aligned}
$$

(e) The following dimension formulas hold

$$
\begin{aligned}
& \operatorname{dim}(\mathcal{N})=\operatorname{dim}(\mathcal{M} \cap \mathcal{N})+\operatorname{dim}\left(\mathcal{N} \circ \mathcal{N}^{\perp}\right)=\operatorname{dim}\left(\mathcal{N} \cap \mathcal{N}^{\perp}\right)+\operatorname{dim}(\mathcal{M} \circ \mathcal{N}), \\
& \operatorname{dim}(\mathcal{N})=\operatorname{dim}(\mathcal{M} \cap \mathcal{N})+\operatorname{dim}\left(\mathcal{N} \circ \mathcal{M}^{\perp}\right)=\operatorname{dim}\left(\mathcal{M}^{\perp} \cap \mathcal{N}\right)+\operatorname{dim}(\mathcal{N} \circ \mathcal{M}), \\
& \operatorname{dim}\left(\mathcal{K}^{\perp}\right)=\operatorname{dim}\left(\mathcal{K}^{\perp} \cap \mathcal{N}\right)+\operatorname{dim}\left(\mathcal{K}^{\perp} \circ \mathcal{N}^{\perp}\right)=\operatorname{dim}\left(\mathcal{K}^{\perp} \cap \mathcal{N}^{\perp}\right)+\operatorname{dim}\left(\mathcal{K}^{\perp} \circ \mathcal{N}\right), \\
& \operatorname{dim}\left(\mathcal{N}^{\perp}\right)=\operatorname{dim}\left(\mathcal{M} \cap \mathcal{N}^{\perp}\right)+\operatorname{dim}\left(\mathcal{N}^{\perp} \circ \mathcal{M}^{\perp}\right)=\operatorname{dim}\left(\mathcal{M}^{\perp} \cap \mathcal{N}^{\perp}\right)+\operatorname{dim}\left(\mathcal{N}^{\perp} \circ \mathcal{M}\right), \\
& \operatorname{dim}(\mathcal{M}+\mathcal{N})=\operatorname{dim}(\mathcal{M} \cap \mathcal{N})+\operatorname{dim}\left(\mathcal{M} \circ \mathcal{N}^{\perp}\right)+\operatorname{dim}\left(\mathcal{N} \circ \mathcal{M}^{\perp}\right), \\
& \operatorname{dim}\left(\mathcal{M}+\mathcal{N}^{\perp}\right)=\operatorname{dim}\left(\mathcal{M} \cap \mathcal{N}^{\perp}\right)+\operatorname{dim}(\mathcal{M} \circ \mathcal{N})+\operatorname{dim}\left(\mathcal{N}^{\perp} \circ \mathcal{M}^{\perp}\right), \\
& \operatorname{dim}\left(\mathcal{M}^{\perp}+\mathcal{N}\right)=\operatorname{dim}\left(\mathcal{M}^{\perp} \cap \mathcal{N}\right)+\operatorname{dim}\left(\mathcal{M}^{\perp} \circ \mathcal{N}^{\perp}\right)+\operatorname{dim}(\mathcal{N} \circ \mathcal{N}), \\
& \operatorname{dim}\left(\mathcal{M}^{\perp}+\mathcal{N}^{\perp}\right)=\operatorname{dim}\left(\mathcal{M}^{\perp} \cap \mathcal{N}^{\perp}\right)+\operatorname{dim}\left(\mathcal{M}^{\perp} \circ \mathcal{N}\right)+\operatorname{dim}\left(\mathcal{N}^{\perp} \circ \mathcal{M}\right) .
\end{aligned}
$$


For convenience of representing the residuals in (2.25)-(2.28), we define a binary operation for two subspaces as follows

$$
\mathcal{M}_{1} \cdot \mathcal{M}_{2} \triangleq \mathcal{M}_{1} \cap\left(\mathcal{M}_{1} \cap \mathcal{M}_{2}\right)^{\perp} \cap\left(\mathcal{M}_{1} \cap \mathcal{M}_{2}^{\perp}\right)^{\perp}=\mathcal{M}_{1} \cap\left(\mathcal{M}_{1}^{\perp}+\mathcal{M}_{2}^{\perp}\right) \cap\left(\mathcal{M}_{1}^{\perp}+\mathcal{M}_{2}\right) .
$$

In term of this notation, we obtain

$$
\begin{aligned}
& \mathcal{M} \cdot \mathcal{N}=\mathcal{M} \cdot \mathcal{N}^{\perp}=\mathcal{M} \cap(\mathcal{M} \cap \mathcal{N})^{\perp} \cap\left(\mathcal{M} \cap \mathcal{N}^{\perp}\right)^{\perp}, \\
& \mathcal{N} \cdot \mathcal{M}=\mathcal{N} \cdot \mathcal{M}^{\perp}=\mathcal{N} \cap(\mathcal{M} \cap \mathcal{N})^{\perp} \cap\left(\mathcal{M}^{\perp} \cap \mathcal{N}\right)^{\perp} \text {, } \\
& \mathcal{M}^{\perp} \cdot \mathcal{N}=\mathcal{M}^{\perp} \cdot \mathcal{N}^{\perp}=\mathcal{M}^{\perp} \cap\left(\mathcal{M}^{\perp} \cap \mathcal{N}\right)^{\perp} \cap\left(\mathcal{M}^{\perp} \cap \mathcal{N}^{\perp}\right)^{\perp} \text {, } \\
& \mathcal{N}^{\perp} \cdot \mathcal{M}=\mathcal{N}^{\perp} \cdot \mathcal{M}^{\perp}=\mathcal{N}^{\perp} \cap\left(\mathcal{M} \cap \mathcal{N}^{\perp}\right)^{\perp} \cap\left(\mathcal{M}^{\perp} \cap \mathcal{N}^{\perp}\right)^{\perp} \text {. }
\end{aligned}
$$

Correspondingly,

$$
\begin{aligned}
& (\mathcal{M} \bullet \mathcal{N})^{\perp}=\left(\mathcal{M} \bullet \mathcal{N}^{\perp}\right)^{\perp}=\mathcal{M}^{\perp}+(\mathcal{M} \cap \mathcal{N})+\left(\mathcal{M} \cap \mathcal{N}^{\perp}\right), \\
& (\mathcal{N} \bullet \mathcal{M})^{\perp}=\left(\mathcal{N} \bullet \mathcal{M}^{\perp}\right)^{\perp}=\mathcal{N}^{\perp}+(\mathcal{M} \cap \mathcal{N})+\left(\mathcal{M}^{\perp} \cap \mathcal{N}\right) \text {, } \\
& \left(\mathcal{M}^{\perp} \bullet \mathcal{N}\right)^{\perp}=\left(\mathcal{M}^{\perp} \cdot \mathcal{N}^{\perp}\right)^{\perp}=\mathcal{M}+\left(\mathcal{M}^{\perp} \cap \mathcal{N}\right)+\left(\mathcal{M}^{\perp} \cap \mathcal{N}^{\perp}\right) \text {, } \\
& \left(\mathcal{N}^{\perp} \cdot \mathcal{M}\right)^{\perp}=\left(\mathcal{N}^{\perp} \bullet \mathcal{M} \mathcal{C}^{\perp}\right)^{\perp}=\mathcal{N}+\left(\mathcal{M} \cap \mathcal{N}^{\perp}\right)+\left(\mathcal{M}^{\perp} \cap \mathcal{N}^{\perp}\right) \text {. }
\end{aligned}
$$

By using the above expressions, we obtain below another group of simultaneous direct sum decomposition identities and their algebraic properties.

Theorem 2.6. Let $\mathcal{M}$ and $\mathcal{N}$ be two linear subspaces of $\mathbb{C}^{m}$. Then the following results hold.

(a) $\mathcal{M}, \mathcal{N}, \mathcal{M}^{\perp}$, and $\mathcal{N}^{\perp}$ can be decomposed as direct sums of three terms

$$
\begin{aligned}
& \mathcal{M}=(\mathcal{M} \cap \mathcal{N}) \oplus\left(\mathcal{M} \cap \mathcal{N}^{\perp}\right) \oplus(\mathcal{M} \bullet \mathcal{N}), \quad \mathcal{N}=(\mathcal{M} \cap \mathcal{N}) \oplus\left(\mathcal{M}^{\perp} \cap \mathcal{N}\right) \oplus(\mathcal{N} \bullet \mathcal{M}), \\
& \mathcal{M}^{\perp}=\left(\mathcal{M}^{\perp} \cap \mathcal{N}\right) \oplus\left(\mathcal{M}^{\perp} \cap \mathcal{N}^{\perp}\right) \oplus\left(\mathcal{M}^{\perp} \cdot \mathcal{N}\right), \mathcal{N}^{\perp}=\left(\mathcal{M} \cap \mathcal{N}^{\perp}\right) \oplus\left(\mathcal{M}^{\perp} \cap \mathcal{N}^{\perp}\right) \oplus\left(\mathcal{N}^{\perp} \cdot \mathcal{M}\right) .
\end{aligned}
$$

Alternatively, $\mathcal{M}, \mathcal{N}, \mathcal{M}^{\perp}$, and $\mathcal{N}^{\perp}$ can be decomposed as intersections of three terms

$$
\begin{aligned}
& \mathcal{M}=(\mathcal{M}+\mathcal{N}) \cap\left(\mathcal{M}+\mathcal{N}^{\perp}\right) \cap\left(\mathcal{M}^{\perp} \bullet \mathcal{N}\right)^{\perp}, \quad \mathcal{N}=(\mathcal{M}+\mathcal{N}) \cap\left(\mathcal{M}^{\perp} \cap \mathcal{N}\right) \cap\left(\mathcal{N}^{\perp} \bullet \mathcal{M}\right)^{\perp}, \\
& \mathcal{M}^{\perp}=\left(\mathcal{M}^{\perp}+\mathcal{N}{ }^{\perp}\right) \cap\left(\mathcal{M}^{\perp}+\mathcal{N}\right) \cap(\mathcal{M} \bullet \mathcal{N})^{\perp}, \mathcal{N}^{\perp}=\left(\mathcal{M}^{\perp}+\mathcal{N}^{\perp}\right) \cap\left(\mathcal{M} \cap \mathcal{N}^{\perp}\right) \cap(\mathcal{N} \bullet \mathcal{M})^{\perp},
\end{aligned}
$$

where

$$
\begin{aligned}
& (\mathcal{M} \bullet \mathcal{N})^{\perp}=\mathcal{M}^{\perp}+(\mathcal{M} \cap \mathcal{N})+\left(\mathcal{M} \cap \mathcal{N}^{\perp}\right), \quad(\mathcal{N} \bullet \mathcal{M})^{\perp}=\mathcal{N}^{\perp}+(\mathcal{M} \cap \mathcal{N})+\left(\mathcal{M} \mathcal{L}^{\perp} \cap \mathcal{N}\right), \\
& \left(\mathcal{M} \mathcal{K}^{\perp} \cdot \mathcal{N}\right)^{\perp}=\mathcal{M}+\left(\mathcal{M}^{\perp} \cap \mathcal{N}\right)^{\perp}+\left(\mathcal{M}^{\perp}+\mathcal{N}^{\perp}\right), \quad\left(\mathcal{N}^{\perp} \bullet \mathcal{M}\right)^{\perp}=\mathcal{N}+\left(\mathcal{M} \cap \mathcal{N}^{\perp}\right)+\left(\mathcal{M} \mathcal{K}^{\perp} \cap \mathcal{N}^{\perp}\right)
\end{aligned}
$$

(b) The following disjoint facts hold

$$
\begin{aligned}
& \left.\left[\left(\mathcal{M} \cap \mathcal{N}^{\perp}\right) \oplus(\mathcal{M} \bullet \mathcal{N})\right] \cap\left[\mathcal{M}^{\perp} \cap \mathcal{N}\right) \oplus(\mathcal{N} \bullet \mathcal{M})\right]=\{0\}, \\
& {[(\mathcal{M} \cap \mathcal{N}) \oplus(\mathcal{M} \bullet \mathcal{N})] \cap\left[\left(\mathcal{M}^{\perp} \cap \mathcal{N}^{\perp}\right) \oplus\left(\mathcal{N}^{\perp} \bullet \mathcal{M}\right)\right]=\{0\},} \\
& {\left[\left(\mathcal{M}^{\perp} \cap \mathcal{N}^{\perp}\right) \oplus\left(\mathcal{M}^{\perp} \bullet \mathcal{N}\right)\right] \cap[(\mathcal{M} \cap \mathcal{N}) \oplus(\mathcal{N} \bullet \mathcal{M})]=\{0\},} \\
& {\left[\left(\mathcal{M}^{\perp} \cap \mathcal{N}\right) \oplus\left(\mathcal{M}^{\perp} \bullet \mathcal{N}\right)\right] \cap\left[\left(\mathcal{M} \cap \mathcal{N}^{\perp}\right) \oplus\left(\mathcal{N}^{\perp} \bullet \mathcal{M}\right)\right]=\{0\} .}
\end{aligned}
$$

(c) The orthogonal projectors onto $\mathcal{M}, \mathcal{N}, \mathcal{M}^{\perp}$, and $\mathcal{N}^{\perp}$ can be decomposed as

$$
\begin{aligned}
& P_{\mathcal{M}}=P_{\mathcal{M} \cap \mathcal{N}}+P_{\mathcal{M} \cap \mathcal{N} \perp}+P_{\mathcal{M} \bullet \mathcal{N}}, \quad P_{\mathcal{N}}=P_{\mathcal{M} \cap \mathcal{N}}+P_{\mathcal{M} \perp \perp \mathcal{N}}+P_{\mathcal{N} \bullet \mathcal{M}}, \\
& P_{\mathcal{M} \perp}=P_{\mathcal{M} \perp \cap \mathcal{N}}+P_{\mathcal{M} \perp \cap \mathcal{N}^{\perp}}+P_{\mathcal{M} \perp \cdot \mathcal{N}}, \quad P_{\mathcal{N}^{\perp}}=P_{\mathcal{M} \cap \mathcal{N}^{\perp}}+P_{\mathcal{M}^{\perp} \cap \mathcal{N}^{\perp}}+P_{\mathcal{N}^{\perp} \bullet \mathcal{M}} ;
\end{aligned}
$$

the following identities hold

$$
P_{\mathcal{M}} P_{\mathcal{N}}=P_{\mathcal{M} \cap \mathcal{N}}+P_{\mathcal{M} \cdot \mathcal{N}} P_{\mathcal{N}} \cdot \mathcal{M}, \quad P_{\mathcal{N}} P_{\mathcal{M}}=P_{\mathcal{M} \cap \mathcal{N}}+P_{\mathcal{N} \cdot \mathcal{M}} P_{\mathcal{M} \cdot \mathcal{N}},
$$




$$
\begin{aligned}
& P_{\mathcal{M}} P_{\mathcal{N}^{\perp}}=P_{\mathcal{M} \cap \mathcal{N}^{\perp}}+P_{\mathcal{M} \bullet \mathcal{N}} P_{\mathcal{N}^{\perp} \bullet \mathcal{M}}, \quad P_{\mathcal{N}^{\perp}} P_{\mathcal{M}}=P_{\mathcal{M} \cap \mathcal{N}^{\perp}}+P_{\mathcal{N}^{\perp} \bullet \mathcal{M}} P_{\mathcal{M} \bullet \mathcal{N}}, \\
& P_{\mathcal{M}^{\perp}} P_{\mathcal{N}}=P_{\mathcal{M}^{\perp} \cap \mathcal{N}}+P_{\mathcal{M}^{\perp} \bullet \mathcal{N}} P_{\mathcal{N} \cdot \mathcal{N}}, \quad P_{\mathcal{N}} P_{\mathcal{M}^{\perp}}=P_{\mathcal{M}^{\perp} \cap \mathcal{N}}+P_{\mathcal{N} \cdot \mathcal{M}} P_{\mathcal{M}^{\perp} \bullet \mathcal{N}}, \\
& P_{\mathcal{M}^{\perp}} P_{\mathcal{N}^{\perp}}=P_{\mathcal{M}^{\perp} \cap \mathcal{N}^{\perp}}+P_{\mathcal{M}^{\perp} \bullet \mathcal{N}} P_{\mathcal{N}^{\perp} \bullet \mathcal{M}}, P_{\mathcal{N}^{\perp}} P_{\mathcal{M}^{\perp}}=P_{\mathcal{M}^{\perp} \cap \mathcal{N}^{\perp}}+P_{\mathcal{N}^{\perp} \bullet \mathcal{M}} P_{\mathcal{M}^{\perp} \bullet \mathcal{N}} ;
\end{aligned}
$$

the following identities hold

$$
\begin{aligned}
& P_{\mathcal{M}} P_{\mathcal{N}}-P_{\mathcal{N}} P_{\mathcal{M}}=P_{\mathcal{M} \cdot \mathcal{N}} P_{\mathcal{N} \cdot \mathcal{N}}-P_{\mathcal{N} \cdot \mathcal{M}} P_{\mathcal{M} \cdot \mathcal{N}}, \\
& P_{\mathcal{M}} P_{\mathcal{N}^{\perp}}-P_{\mathcal{N} \perp} P_{\mathcal{M}}=P_{\mathcal{M} \cdot \mathcal{N}} P_{\mathcal{N}^{\perp} \cdot \mathcal{M}}-P_{\mathcal{N} \perp \cdot \mathcal{M}} P_{\mathcal{M}} \cdot \mathcal{N}, \\
& P_{\mathcal{M}}{ } P_{\mathcal{N}}-P_{\mathcal{N}} P_{\mathcal{M} \perp}=P_{\mathcal{M} \perp \cdot \mathcal{N}} P_{\mathcal{N} \cdot \mathcal{M}}-P_{\mathcal{N} \cdot{ }_{\mathcal{M}}} P_{\mathcal{M}}{ }^{\perp \cdot \mathcal{N}}, \\
& P_{\mathcal{M} \perp} P_{\mathcal{N}^{\perp}}-P_{\mathcal{N}^{\perp}} P_{\mathcal{M}^{\perp}}=P_{\mathcal{M} \perp \cdot \mathcal{N}} P_{\mathcal{N}^{\perp} \bullet \mathcal{M}}-P_{\mathcal{N}^{\perp} \bullet \mathfrak{M}} P_{\mathcal{M}^{\perp} \bullet \mathcal{N}} \\
& P_{\mathcal{M}} P_{\mathcal{N}}-P_{\mathcal{N}} P_{\mathcal{M}}=P_{\mathcal{N} \perp} P_{\mathcal{M}}-P_{\mathcal{M}} P_{\mathcal{N} \perp}=P_{\mathcal{N} \perp} P_{\mathcal{M}}-P_{\mathcal{M}} P_{\mathcal{N} \perp}=P_{\mathcal{M} \perp} P_{\mathcal{N} \perp}-P_{\mathcal{N}^{\perp}} P_{\mathcal{M} \perp} ;
\end{aligned}
$$

and the following commutative equalities hold

$$
\begin{aligned}
& \left(P_{\mathcal{M}}-P_{\mathcal{M} \cdot \mathcal{N}}\right)\left(P_{\mathcal{N}}-P_{\mathcal{N} \cdot \mathcal{M}}\right)=\left(P_{\mathcal{N}}-P_{\mathcal{N} \cdot \mathcal{M}}\right)\left(P_{\mathcal{M}}-P_{\mathcal{X} \cdot \mathcal{N}}\right), \\
& \left(P_{\mathcal{K}}-P_{\mathcal{M} \cdot \mathcal{N}}\right)\left(P_{\mathcal{N}^{\perp}}-P_{\mathcal{N} \perp \cdot \mathcal{N}}\right)=\left(P_{\mathcal{N}^{\perp}}-P_{\mathcal{N} \perp \cdot \mathcal{M}}\right)\left(P_{\mathcal{M}}-P_{\mathcal{M} \bullet \mathcal{N}}\right), \\
& \left(P_{\mathcal{M}^{\perp}}-P_{\mathcal{M}^{\perp} \bullet \mathcal{N}}\right)\left(P_{\mathcal{N}}-P_{\mathcal{N} \bullet \mathcal{N}}\right)=\left(P_{\mathcal{N}}-P_{\mathcal{N} \bullet \mathcal{M}}\right)\left(P_{\mathcal{M}^{\perp}}-P_{\mathcal{M}^{\perp} \bullet \mathcal{N}}\right), \\
& \left(P_{\mathcal{M}^{\perp}}-P_{\mathcal{M}^{\perp} \bullet \mathcal{N}}\right)\left(P_{\mathcal{N}^{\perp}}-P_{\mathcal{N}^{\perp} \bullet \mathcal{N}}\right)=\left(P_{\mathcal{N}^{\perp}}-P_{\mathcal{N}^{\perp} \bullet \mathcal{M}}\right)\left(P_{\mathcal{M}^{\perp}}-P_{\mathcal{M}^{\perp} \bullet \mathcal{N}}\right) \text {. }
\end{aligned}
$$

(d) The following dimension formulas hold

$$
\begin{aligned}
& \operatorname{dim}(\mathcal{N})=\operatorname{dim}(\mathcal{M} \cap \mathcal{N})+\operatorname{dim}\left(\mathcal{M} \cap \mathcal{N}^{\perp}\right)+\operatorname{dim}(\mathcal{M} \bullet \mathcal{N}), \\
& \operatorname{dim}(\mathcal{N})=\operatorname{dim}(\mathcal{M} \cap \mathcal{N})+\operatorname{dim}\left(\mathcal{M}^{\perp} \cap \mathcal{N}\right)+\operatorname{dim}(\mathcal{M} \bullet \mathcal{N}), \\
& \operatorname{dim}\left(\mathcal{M}^{\perp}\right)=\operatorname{dim}\left(\mathcal{M}^{\perp} \cap \mathcal{N}\right)+\operatorname{dim}\left(\mathcal{M}^{\perp} \cap \mathcal{N}^{\perp}\right)+\operatorname{dim}\left(\mathcal{M}^{\perp} \bullet \mathcal{N}\right), \\
& \operatorname{dim}\left(\mathcal{N}^{\perp}\right)=\operatorname{dim}\left(\mathcal{M} \cap \mathcal{N}^{\perp}\right)+\operatorname{dim}\left(\mathcal{M}^{\perp} \cap \mathcal{N}^{\perp}\right)+\operatorname{dim}\left(\mathcal{N}^{\perp} \bullet \mathcal{M}\right), \\
& \operatorname{dim}(\mathcal{N})+\operatorname{dim}\left(\mathcal{N}^{\perp} \cap \mathcal{N}\right)=\operatorname{dim}(\mathcal{N})+\operatorname{dim}\left(\mathcal{M} \cap \mathcal{N}^{\perp}\right), \\
& \operatorname{dim}(\mathcal{N})+\operatorname{dim}\left(\mathcal{M}^{\perp} \cap \mathcal{N}^{\perp}\right)=\operatorname{dim}\left(\mathcal{N}^{\perp}\right)+\operatorname{dim}(\mathcal{M} \cap \mathcal{N}), \\
& \operatorname{dim}\left(\mathcal{M}^{\perp}\right)+\operatorname{dim}(\mathcal{M} \cap \mathcal{N})=\operatorname{dim}(\mathcal{N})+\operatorname{dim}\left(\mathcal{M}^{\perp} \cap \mathcal{N}^{\perp}\right), \\
& \operatorname{dim}\left(\mathcal{M}^{\perp}\right)+\operatorname{dim}\left(\mathcal{K} \cap \mathcal{N}^{\perp}\right)=\operatorname{dim}\left(\mathcal{N}^{\perp}\right)+\operatorname{dim}\left(\mathcal{M}^{\perp} \cap \mathcal{N}\right), \\
& \operatorname{dim}(\mathcal{N} \bullet \mathcal{N})=\operatorname{dim}(\mathcal{N} \bullet \mathcal{M})=\operatorname{dim}\left(\mathcal{M}^{\perp} \bullet \mathcal{N}\right)=\operatorname{dim}\left(\mathcal{N}^{\perp} \bullet \mathcal{M}\right) .
\end{aligned}
$$

(e) The following five-term simultaneous direct sum decomposition identities hold

$$
\begin{aligned}
& \mathcal{M}+\mathcal{N}=(\mathcal{M} \cap \mathcal{N}) \oplus\left(\mathcal{M} \cap \mathcal{N}^{\perp}\right) \oplus\left(\mathcal{K}^{\perp} \cap \mathcal{N}\right) \oplus(\mathcal{K} \bullet \mathcal{N}) \oplus(\mathcal{N} \bullet \mathcal{M}), \\
& \mathcal{M}+\mathcal{N}^{\perp}=(\mathcal{M} \cap \mathcal{N}) \oplus\left(\mathcal{M} \cap \mathcal{N}^{\perp}\right) \oplus\left(\mathcal{M}^{\perp} \cap \mathcal{N}^{\perp}\right) \oplus(\mathcal{M} \bullet \mathcal{N}) \oplus\left(\mathcal{N}^{\perp} \bullet \mathcal{N}\right), \\
& \mathcal{M}^{\perp}+\mathcal{N}=(\mathcal{M} \cap \mathcal{N}) \oplus\left(\mathcal{K}^{\perp} \cap \mathcal{N}\right) \oplus\left(\mathcal{K}^{\perp} \cap \mathcal{N}^{\perp}\right) \oplus\left(\mathcal{K}^{\perp} \bullet \mathcal{N}\right) \oplus(\mathcal{N} \cdot \mathcal{N}) \text {, } \\
& \mathcal{M}^{\perp}+\mathcal{N}^{\perp}=\left(\mathcal{K} \cap \mathcal{N}^{\perp}\right) \oplus\left(\mathcal{M}^{\perp} \cap \mathcal{N}\right) \oplus\left(\mathcal{M}^{\perp} \cap \mathcal{N}^{\perp}\right) \oplus\left(\mathcal{M}^{\perp} \bullet \mathcal{N}\right) \oplus\left(\mathcal{N}^{\perp} \bullet \mathcal{M}\right) \text {. }
\end{aligned}
$$

(f) The following orthogonal projector decomposition identities hold

$$
\begin{aligned}
& P_{\mathcal{M}+\mathcal{N}}=P_{\mathcal{M} \cap \mathcal{N}}+P_{\mathcal{M} \cap \mathcal{N}^{\perp}}+P_{\mathcal{M} \perp \cap \mathcal{N}}+P_{(\mathcal{M} \bullet \mathcal{N})+(\mathcal{N} \bullet \mathcal{M N})} \text {, } \\
& P_{\mathcal{M}+\mathcal{N} \perp}=P_{\mathcal{M} \cap \mathcal{N}}+P_{\mathcal{M} \cap \mathcal{N} \perp}+P_{\mathcal{M} \perp \cap \mathcal{N} \perp}+P_{(\mathcal{M} \cdot \mathcal{N})+(\mathcal{N} \perp \cdot \mathcal{N})} \text {, } \\
& \left.P_{\mathcal{M}^{\perp}+\mathcal{N}}=P_{\mathcal{M} \cap \mathcal{N}}+P_{\mathcal{M}^{\perp} \cap \mathcal{N}}+P_{\mathcal{M}^{\perp} \cap \mathcal{N}^{\perp}}+P_{(\mathcal{M} \perp} \cdot \mathcal{N}\right)+(\mathcal{N} \bullet \mathcal{M N}) \text {, } \\
& P_{\mathcal{M}^{\perp}+\mathcal{N}^{\perp}}=P_{\mathcal{M} \cap \mathcal{N}^{\perp}}+P_{\mathcal{M}^{\perp} \cap \mathcal{N}}+P_{\mathcal{M}^{\perp} \cap \mathcal{N}^{\perp}}+P_{\left(\mathcal{M}^{\perp} \cdot \mathcal{N}\right)+(\mathcal{N} \perp \cdot \bullet \mathcal{N})} \cdot
\end{aligned}
$$

(g) The following dimension identities hold

$$
\operatorname{dim}(\mathcal{M}+\mathcal{N})=\operatorname{dim}(\mathcal{M} \cap \mathcal{N})+\operatorname{dim}\left(\mathcal{M} \cap \mathcal{N}^{\perp}\right)+\operatorname{dim}\left(\mathcal{M}^{\perp} \cap \mathcal{N}\right)+\operatorname{dim}(\mathcal{N} \bullet \mathcal{N})+\operatorname{dim}(\mathcal{N} \bullet \mathcal{M})
$$




$$
\begin{aligned}
& \operatorname{dim}\left(\mathcal{M}+\mathcal{N}^{\perp}\right)=\operatorname{dim}(\mathcal{M} \cap \mathcal{N})+\operatorname{dim}\left(\mathcal{M} \cap \mathcal{N}^{\perp}\right)+\operatorname{dim}\left(\mathcal{M}^{\perp} \cap \mathcal{N}^{\perp}\right)+\operatorname{dim}(\mathcal{M} \bullet \mathcal{N})+\operatorname{dim}\left(\mathcal{N}^{\perp} \bullet \mathcal{M}\right), \\
& \operatorname{dim}\left(\mathcal{M}^{\perp}+\mathcal{N}\right)=\operatorname{dim}(\mathcal{M} \cap \mathcal{N})+\operatorname{dim}\left(\mathcal{M}^{\perp} \cap \mathcal{N}\right)+\operatorname{dim}\left(\mathcal{M}^{\perp} \cap \mathcal{N}^{\perp}\right)+\operatorname{dim}\left(\mathcal{M}^{\perp} \bullet \mathcal{N}\right)+\operatorname{dim}(\mathcal{N} \bullet \mathcal{M}), \\
& \operatorname{dim}\left(\mathcal{M}^{\perp}+\mathcal{N}^{\perp}\right)=\operatorname{dim}\left(\mathcal{M} \cap \mathcal{N}^{\perp}\right)+\operatorname{dim}\left(\mathcal{M}^{\perp} \cap \mathcal{N}\right)+\operatorname{dim}\left(\mathcal{M}^{\perp} \cap \mathcal{N}^{\perp}\right)+\operatorname{dim}\left(\mathcal{M}^{\perp} \bullet \mathcal{N}\right) \\
& +\operatorname{dim}\left(\mathcal{N}^{\perp} \bullet \mathcal{M}\right) \text {, } \\
& \operatorname{dim}(\mathcal{M}+\mathcal{N})+\operatorname{dim}\left(\mathcal{N}^{\perp}\right)=\operatorname{dim}\left(\mathcal{M}^{\perp}+\mathcal{N}^{\perp}\right)+\operatorname{dim}(\mathcal{N}), \\
& \operatorname{dim}\left(\mathcal{N}+\mathcal{N}^{\perp}\right)+\operatorname{dim}(\mathcal{N})=\operatorname{dim}\left(\mathcal{M}^{\perp}+\mathcal{N}\right)+\operatorname{dim}(\mathcal{N}), \\
& \operatorname{dim}\left(\mathcal{M}^{\perp}+\mathcal{N}\right)+\operatorname{dim}\left(\mathcal{N}^{\perp}\right)=\operatorname{dim}\left(\mathcal{M}+\mathcal{N}^{\perp}\right)+\operatorname{dim}\left(\mathcal{M}^{\perp}\right), \\
& \operatorname{dim}\left(\mathcal{M}^{\perp}+\mathcal{N}^{\perp}\right)+\operatorname{dim}(\mathcal{N})=\operatorname{dim}(\mathcal{M}+\mathcal{N})+\operatorname{dim}\left(\mathcal{M}^{\perp}\right) \text {. }
\end{aligned}
$$

Proof. Note from (2.12) that

$$
\begin{aligned}
& {\left[(\mathcal{M} \cap \mathcal{N}) \oplus\left(\mathcal{M} \cap \mathcal{N}^{\perp}\right)\right] \oplus\left[(\mathcal{M} \cap \mathcal{N}) \oplus\left(\mathcal{M} \cap \mathcal{N}^{\perp}\right)\right]^{\perp}=\mathbb{C}^{m},} \\
& \mathcal{M} \supseteq(\mathcal{M} \cap \mathcal{N}) \oplus\left(\mathcal{M} \cap \mathcal{N}^{\perp}\right) .
\end{aligned}
$$

Hence, we obtain from (2.20) that

$$
\begin{aligned}
\mathcal{M} & =\mathcal{M} \cap\left\{\left[(\mathcal{M} \cap \mathcal{N}) \oplus\left(\mathcal{M} \cap \mathcal{N}^{\perp}\right)\right] \oplus\left[(\mathcal{M} \cap \mathcal{N}) \oplus\left(\mathcal{M} \cap \mathcal{N}^{\perp}\right)\right]^{\perp}\right\} \\
& =\left[(\mathcal{M} \cap \mathcal{N}) \oplus\left(\mathcal{M} \cap \mathcal{N}^{\perp}\right)\right] \oplus\left\{\mathcal{M} \cap\left[(\mathcal{M} \cap \mathcal{N}) \oplus\left(\mathcal{M} \cap \mathcal{N}^{\perp}\right)\right]^{\perp}\right\} \\
& =\left[(\mathcal{M} \cap \mathcal{N}) \oplus\left(\mathcal{M} \cap \mathcal{N}^{\perp}\right)\right] \oplus\left[\mathcal{M} \cap(\mathcal{M} \cap \mathcal{N})^{\perp} \cap\left(\mathcal{M} \cap \mathcal{N}^{\perp}\right)^{\perp}\right] \\
& =(\mathcal{M} \cap \mathcal{N}) \oplus\left(\mathcal{M} \cap \mathcal{N}^{\perp}\right) \oplus(\mathcal{M} \bullet \mathcal{N}),
\end{aligned}
$$

establishing the first equality in (a), where three terms on the right-hand side are obviously pairwise orthogonal. The other three equalities in (a) can be shown similarly. Also note that

$$
(\mathcal{M} \bullet \mathcal{N}) \cap(\mathcal{N} \cdot \mathcal{M})=(\mathcal{M} \cap \mathcal{N}) \cap(\mathcal{M} \cap \mathcal{N})^{\perp} \cap\left(\mathcal{M} \cap \mathcal{N}^{\perp}\right)^{\perp} \cap\left(\mathcal{M}^{\perp} \cap \mathcal{N}\right)^{\perp}=\{0\},
$$

establishing the first identity in (b), so that

$$
(\mathcal{M} \cdot \mathcal{N})+(\mathcal{N} \cdot \mathcal{M})=(\mathcal{M} \cdot \mathcal{N}) \oplus(\mathcal{N} \bullet \mathcal{M}) .
$$

Adding the first and second equalities in (a) and applying the above equality lead to the first equality in (e). The remaining equalities in (e) can be established by a similar approach.

Taking orthogonal projectors of both sides of (a) and applying orthogonality among the three terms on the right-hand sides leads to the first group of equalities in (c). The second and third groups of equalities in (c) follow from first group of equalities in (c), $P_{\mathcal{M}^{\perp}}=I_{m}-P_{\mathcal{M}}$ and $P_{\mathcal{N}^{\perp}}=I_{m}-P_{\mathcal{N}}$.

Taking dimensions of both sides of (a) leads to the first four equalities in (d), as well as the remaining equalities in (d).

Taking orthogonal projectors of both sides of four equalities in (e) leads to (f).

Taking dimensions of both sides of four equalities in (e) leads to ( $\mathrm{g}$ ).

We next derive more facts about two subspaces and their operations. The following concept on the commutator of two subspaces is well known; see e.g., [93].

Definition 2.7. Let $\mathcal{M}$ and $\mathcal{N}$ be two linear subspaces of $\mathbb{C}^{m}$. The commutator of $\mathcal{N}$ and $\mathcal{N}$ is defined to be

$$
\operatorname{Comm}(\mathcal{M}, \mathcal{N})=(\mathcal{M} \cap \mathcal{N}) \oplus\left(\mathcal{M} \cap \mathcal{N}^{\perp}\right) \oplus\left(\mathcal{M}^{\perp} \cap \mathcal{N}\right) \oplus\left(\mathcal{M}^{\perp} \cap \mathcal{N}^{\perp}\right) .
$$

Substituting Theorem 2.6(e) into (2.29)-(2.32) and using Definition 2.7, we obtain the following consequences.

Theorem 2.8. Let $\mathcal{N}$ and $\mathcal{N}$ be two linear subspaces of $\mathbb{C}^{m}$. Then, the following results hold.

(a) The dimension of $\operatorname{Comm}(\mathcal{M}, \mathcal{N})$ is

$$
\operatorname{dim}[\operatorname{Comm}(\mathcal{N}, \mathcal{N})]=\operatorname{dim}(\mathcal{M} \cap \mathcal{N})+\operatorname{dim}\left(\mathcal{M} \cap \mathcal{N}^{\perp}\right)+\operatorname{dim}\left(\mathcal{M}^{\perp} \cap \mathcal{N}\right)+\operatorname{dim}\left(\mathcal{M}^{\perp} \cap \mathcal{N}^{\perp}\right)
$$


(b) $\mathbb{C}^{m}$ can be written as the following four five-term direct sums

$$
\begin{aligned}
& \mathbb{C}^{m}=\operatorname{Comm}(\mathcal{M}, \mathcal{N}) \oplus(\mathcal{M} \bullet \mathcal{N}) \oplus(\mathcal{N} \cdot \mathcal{M})=\operatorname{Comm}(\mathcal{M}, \mathcal{N}) \oplus(\mathcal{M} \bullet \mathcal{N}) \oplus\left(\mathcal{N}^{\perp} \bullet \mathcal{M}\right) \\
& =\operatorname{Comm}(\mathcal{N}, \mathcal{N}) \oplus\left(\mathcal{M}^{\perp} \cdot \mathcal{N}\right) \oplus(\mathcal{N} \cdot \mathcal{N})=\operatorname{Comm}(\mathcal{M}, \mathcal{N}) \oplus\left(\mathcal{M}^{\perp} \cdot \mathcal{N}\right) \oplus\left(\mathcal{N}^{\perp} \cdot \mathcal{M}\right) .
\end{aligned}
$$

It can be seen from Theorems 2.6(a) and 2.8(b) that all possible ordinary operations of two subspaces $\mathcal{M}$ and $\mathcal{N}$ in $\mathbb{C}^{m}$ can be decomposed as the direct sums of the following eight canonical subspaces:

$$
\mathcal{M} \cap \mathcal{N}, \mathcal{M} \cap \mathcal{N}^{\perp}, \mathcal{M}^{\perp} \cap \mathcal{N}, \mathcal{M}^{\perp} \cap \mathcal{N}^{\perp}, \mathcal{M} \bullet \mathcal{N}, \mathcal{N} \bullet \mathcal{M}, \mathcal{M}^{\perp} \cdot \mathcal{N}, \mathcal{M}^{\perp} \cdot \mathcal{N} .
$$

For convenience, we call the four seminal expansion formulas in Theorem 2.6(a) the joint direct sum decomposition identities (JDSDIs) of two subspaces in $\mathbb{C}^{m}$. The closed-form expressions of the four JDSDIs and their subsequences have a great advantage in dealing with various problems related to two subspaces, and a huge amount of consequences, as common exercises in linear algebra, can be derived from these fundamental JDSDIs only using usual symbolic operations of subspaces.

Theorem 2.9. Let $\mathcal{N}$ and $\mathcal{N}$ be two linear subspaces of $\mathbb{C}^{m}$.

(a) The following results hold

$$
\begin{aligned}
& \mathcal{M} \circ \mathcal{N}=(\mathcal{M} \cap \mathcal{N}) \oplus(\mathcal{M} \bullet \mathcal{N}), \quad \mathcal{M} \circ \mathcal{N}^{\perp}=\left(\mathcal{M} \cap \mathcal{N}^{\perp}\right) \oplus(\mathcal{M} \bullet \mathcal{N}) \text {, } \\
& \mathcal{M}^{\perp} \circ \mathcal{N}=\left(\mathcal{M}^{\perp} \cap \mathcal{N}\right) \oplus\left(\mathcal{M}^{\perp} \bullet \mathcal{N}\right), \mathcal{M}^{\perp} \circ \mathcal{N}^{\perp}=\left(\mathcal{M}^{\perp} \cap \mathcal{N}^{\perp}\right) \oplus\left(\mathcal{M}^{\perp} \bullet \mathcal{N}\right) \text {, } \\
& \mathcal{N} \circ \mathcal{M}=(\mathcal{N} \cap \mathcal{M}) \oplus(\mathcal{N} \bullet \mathcal{M}), \quad \mathcal{N} \circ \mathcal{M}^{\perp}=\left(\mathcal{N} \cap \mathcal{M}^{\perp}\right) \oplus(\mathcal{N} \bullet \mathcal{M}) \text {, } \\
& \mathcal{N}^{\perp} \circ \mathcal{M}=\left(\mathcal{N}^{\perp} \cap \mathcal{M}\right) \oplus\left(\mathcal{N}^{\perp} \bullet \mathcal{M}\right), \mathcal{N}^{\perp} \circ \mathcal{M}^{\perp}=\left(\mathcal{N}^{\perp} \cap \mathcal{M}^{\perp}\right) \oplus\left(\mathcal{N}^{\perp} \bullet \mathcal{M}\right) \text {. }
\end{aligned}
$$

(b) The following results hold

$$
\begin{aligned}
& (\mathcal{M} \circ \mathcal{N}) \cap(\mathcal{N} \circ \mathcal{M})=\mathcal{M} \cap \mathcal{N}, \quad\left(\mathcal{M} \circ \mathcal{N}^{\perp}\right) \cap\left(\mathcal{N}^{\perp} \circ \mathcal{M}\right)=\mathcal{M} \cap \mathcal{N}^{\perp}, \\
& \left(\mathcal{M}^{\perp} \circ \mathcal{N}\right) \cap\left(\mathcal{N} \circ \mathcal{M}^{\perp}\right)=\mathcal{M}^{\perp} \cap \mathcal{N}, \quad\left(\mathcal{M}^{\perp} \circ \mathcal{N}^{\perp}\right) \cap\left(\mathcal{N}^{\perp} \circ \mathcal{M}^{\perp}\right)=\mathcal{M}^{\perp} \cap \mathcal{N}^{\perp}, \\
& (\mathcal{M} \circ \mathcal{N}) \cap\left(\mathcal{M} \circ \mathcal{N}^{\perp}\right)=\mathcal{M} \bullet \mathcal{N}, \quad(\mathcal{N} \circ \mathcal{M}) \cap\left(\mathcal{N} \circ \mathcal{M}^{\perp}\right)=\mathcal{N} \bullet \mathcal{M}, \\
& \left(\mathcal{M}^{\perp} \circ \mathcal{N}\right) \cap\left(\mathcal{M}^{\perp} \circ \mathcal{N}^{\perp}\right)=\mathcal{M}^{\perp} \bullet \mathcal{N}, \quad\left(\mathcal{N}^{\perp} \circ \mathcal{M}\right) \cap\left(\mathcal{N}^{\perp} \circ \mathcal{M}^{\perp}\right)=\mathcal{N}^{\perp} \bullet \mathcal{M} .
\end{aligned}
$$

Proof. The eight equalities in (a) follow from comparing Theorem 2.5(a) with Theorem 2.6(a). It is easy to see

$$
(\mathcal{M} \circ \mathcal{N}) \cap(\mathcal{N} \circ \mathcal{M})=(\mathcal{M} \cap \mathcal{N}) \cap\left(\mathcal{M} \cap \mathcal{N}^{\perp}\right)^{\perp} \cap\left(\mathcal{M}^{\perp} \cap \mathcal{N}\right)^{\perp} \subseteq \mathcal{M} \cap \mathcal{N} .
$$

Also note from (2.16) that

$$
\begin{aligned}
& (\mathcal{M} \cap \mathcal{N}) \cap\left(\mathcal{M} \cap \mathcal{N}^{\perp}\right)^{\perp}=(\mathcal{M} \cap \mathcal{N}) \cap\left(\mathcal{M}^{\perp}+\mathcal{N}\right) \supseteq\left(\mathcal{M} \cap \mathcal{N} \cap \mathcal{M}^{\perp}\right)+(\mathcal{M} \cap \mathcal{N} \cap \mathcal{N})=\mathcal{M} \cap \mathcal{N} \\
& (\mathcal{M} \cap \mathcal{N}) \cap\left(\mathcal{M}^{\perp} \cap \mathcal{N}\right)^{\perp}=(\mathcal{M} \cap \mathcal{N}) \cap\left(\mathcal{M}+\mathcal{N}^{\perp}\right) \supseteq(\mathcal{M} \cap \mathcal{N} \cap \mathcal{M})+\left(\mathcal{M} \cap \mathcal{N} \cap \mathcal{N}^{\perp}\right)=\mathcal{M} \cap \mathcal{N} .
\end{aligned}
$$

So that

$$
(\mathcal{M} \circ \mathcal{N}) \cap(\mathcal{N} \circ \mathcal{M})=(\mathcal{M} \cap \mathcal{N}) \cap\left(\mathcal{M} \cap \mathcal{N}^{\perp}\right)^{\perp} \cap\left(\mathcal{M}^{\perp} \cap \mathcal{N}\right)^{\perp} \supseteq \mathcal{M} \cap \mathcal{N} .
$$

Combining (2.59) and (2.60) gives the first equality in (b). The second to fourth equalities in (b) can be shown similarly. The remaining four equalities follow directly from (2.41)-(2.48) and (2.49)-(2.52).

From Theorems 2.5 and 2.6, we further obtain the following result.

Theorem 2.10. Let $\mathcal{M}$ and $\mathcal{N}$ be two linear subspaces of $\mathbb{C}^{m}$. Then, the following results hold.

(a) $\mathcal{M}, \mathcal{N}, \mathcal{M}^{\perp}$, and $\mathcal{N}^{\perp}$ can simultaneously be decomposed as direct sums of three terms

$$
\mathcal{M}=((\mathcal{M} \circ \mathcal{N}) \cap(\mathcal{N} \circ \mathcal{M})) \oplus\left(\left(\mathcal{M} \circ \mathcal{N}^{\perp}\right) \cap\left(\mathcal{N}^{\perp} \circ \mathcal{M}\right)\right) \oplus\left((\mathcal{M} \circ \mathcal{N}) \cap\left(\mathcal{M} \circ \mathcal{N}^{\perp}\right)\right),
$$




$$
\begin{aligned}
& \mathcal{N}=((\mathcal{M} \circ \mathcal{N}) \cap(\mathcal{N} \circ \mathcal{M})) \oplus\left(\left(\mathcal{M}^{\perp} \circ \mathcal{N}\right) \cap\left(\mathcal{N} \circ \mathcal{M}^{\perp}\right)\right) \oplus\left((\mathcal{N} \circ \mathcal{M}) \cap\left(\mathcal{N} \circ \mathcal{M}^{\perp}\right)\right), \\
& \mathcal{M}^{\perp}=\left(\left(\mathcal{M} \mathcal{M}^{\perp} \circ \mathcal{N}\right) \cap\left(\mathcal{N} \circ \mathcal{M}^{\perp}\right)\right) \oplus\left(\left(\mathcal{M}^{\perp} \circ \mathcal{N}^{\perp}\right) \cap\left(\mathcal{N}^{\perp} \circ \mathcal{M}^{\perp}\right)\right) \oplus\left(\left(\mathcal{M}^{\perp} \circ \mathcal{N}\right) \cap\left(\mathcal{M}^{\perp} \circ \mathcal{N}^{\perp}\right)\right), \\
& \mathcal{N}^{\perp}=\left(\left(\mathcal{M} \circ \mathcal{N}^{\perp}\right) \cap\left(\mathcal{N}^{\perp} \circ \mathcal{M}\right)\right) \oplus\left(\left(\mathcal{M}^{\perp} \circ \mathcal{N}^{\perp}\right) \cap\left(\mathcal{N}^{\perp} \circ \mathcal{M}^{\perp}\right)\right) \oplus\left(\left(\mathcal{N}^{\perp} \circ \mathcal{M}\right) \cap\left(\mathcal{N}^{\perp} \circ \mathcal{M}^{\perp}\right)\right) .
\end{aligned}
$$

Alternatively, $\mathcal{M}, \mathcal{N}, \mathcal{M}^{\perp}$, and $\mathcal{N}^{\perp}$ can be decomposed as intersections of three terms

$$
\begin{aligned}
& \left.\mathcal{M}=\left(\left(\mathcal{M}^{\perp} \circ \mathcal{N}\right)^{\perp}+\left(\mathcal{N} \circ \mathcal{M}^{\perp}\right)^{\perp}\right) \cap\left(\left(\mathcal{M}^{\perp} \circ \mathcal{N}^{\perp}\right)^{\perp}+\left(\mathcal{N}^{\perp} \circ \mathcal{M}^{\perp}\right)^{\perp}\right)\right) \cap\left(\left(\mathcal{M}^{\perp} \circ \mathcal{N}\right)^{\perp}\right. \\
& \left.+\left(\mathcal{M}^{\perp} \circ \mathcal{N}^{\perp}\right)^{\perp}\right) \text {, } \\
& \mathcal{N}=\left(\left(\mathcal{M} \circ \mathcal{N}^{\perp}\right)^{\perp}+\left(\mathcal{N}^{\perp} \circ \mathcal{M}\right)^{\perp}\right) \cap\left(\left(\mathcal{M}^{\perp} \circ \mathcal{N}^{\perp}\right)^{\perp}+\left(\mathcal{N}^{\perp} \circ \mathcal{M}^{\perp}\right)^{\perp}\right) \cap\left(\left(\mathcal{N}^{\perp} \circ \mathcal{M}\right)^{\perp}\right. \\
& \left.+\left(\mathcal{N}^{\perp} \circ \mathcal{M}^{\perp}\right)^{\perp}\right) \\
& \mathcal{M}^{\perp}=\left((\mathcal{M} \circ \mathcal{N})^{\perp}+(\mathcal{N} \circ \mathcal{M})^{\perp}\right) \cap\left(\left(\mathcal{M} \circ \mathcal{N}^{\perp}\right)^{\perp}+\left(\mathcal{N}^{\perp} \circ \mathcal{M}\right)^{\perp}\right) \cap\left((\mathcal{M} \circ \mathcal{N})^{\perp}+\left(\mathcal{M} \circ \mathcal{N} \mathcal{N}^{\perp}\right)^{\perp}\right) \text {, } \\
& \mathcal{N}^{\perp}=\left((\mathcal{M} \circ \mathcal{N})^{\perp}+(\mathcal{N} \circ \mathcal{M})^{\perp}\right) \cap\left(\left(\mathcal{M}^{\perp} \circ \mathcal{N}\right)^{\perp}+\left(\mathcal{N} \circ \mathcal{M}^{\perp}\right)^{\perp}\right) \cap\left((\mathcal{N} \circ \mathcal{M})^{\perp}+\left(\mathcal{N} \circ \mathcal{M}^{\perp}\right)^{\perp}\right) .
\end{aligned}
$$

(b) The sums $\mathcal{M}+\mathcal{N}, \mathcal{M}+\mathcal{N}^{\perp}, \mathcal{M}^{\perp}+\mathcal{N}$, and $\mathcal{M}^{\perp}+\mathcal{N}^{\perp}$ can simultaneously be written as five-term direct sums

$$
\begin{aligned}
& \mathcal{M}+\mathcal{N}=((\mathcal{M} \circ \mathcal{N}) \cap(\mathcal{N} \circ \mathcal{M})) \oplus\left(\left(\mathcal{M} \circ \mathcal{N}^{\perp}\right) \cap\left(\mathcal{N}^{\perp} \circ \mathcal{M}\right)\right) \\
& \oplus\left(\left(\mathcal{M}^{\perp} \circ \mathcal{N}\right) \cap\left(\mathcal{N} \circ \mathcal{M}^{\perp}\right)\right) \oplus\left((\mathcal{M} \circ \mathcal{N}) \cap\left(\mathcal{M} \circ \mathcal{N}^{\perp}\right)\right) \\
& \oplus\left((\mathcal{N} \circ \mathcal{M}) \cap\left(\mathcal{N} \circ \mathcal{M}^{\perp}\right)\right) \\
& \mathcal{M}+\mathcal{N}^{\perp}=((\mathcal{M} \circ \mathcal{N}) \cap(\mathcal{N} \circ \mathcal{M})) \oplus\left(\left(\mathcal{M} \circ \mathcal{N}^{\perp}\right) \cap\left(\mathcal{N}^{\perp} \circ \mathcal{M}\right)\right) \\
& \oplus\left(\left(\mathcal{M}^{\perp} \circ \mathcal{N}^{\perp}\right) \cap\left(\mathcal{N}^{\perp} \circ \mathcal{M}^{\perp}\right)\right) \oplus\left((\mathcal{M} \circ \mathcal{N}) \cap\left(\mathcal{M} \circ \mathcal{N}^{\perp}\right)\right) \\
& \oplus\left(\left(\mathcal{N}^{\perp} \circ \mathcal{M}\right) \cap\left(\mathcal{N}^{\perp} \circ \mathcal{M}^{\perp}\right)\right) \\
& \mathcal{M}^{\perp}+\mathcal{N}=((\mathcal{M} \circ \mathcal{N}) \cap(\mathcal{N} \circ \mathcal{M})) \oplus\left(\left(\mathcal{M}^{\perp} \circ \mathcal{N}\right) \cap\left(\mathcal{N} \circ \mathcal{M}^{\perp}\right)\right) \\
& \oplus\left(\left(\mathcal{M}^{\perp} \circ \mathcal{N}^{\perp}\right) \cap\left(\mathcal{N}^{\perp} \circ \mathcal{M}^{\perp}\right)\right) \oplus\left(\left(\mathcal{M}^{\perp} \circ \mathcal{N}\right) \cap\left(\mathcal{M}^{\perp} \circ \mathcal{N}^{\perp}\right)\right) \\
& \oplus\left((\mathcal{N} \circ \mathcal{M}) \cap\left(\mathcal{N} \circ \mathcal{M}^{\perp}\right)\right) \text {, } \\
& \mathcal{M}^{\perp}+\mathcal{N}^{\perp}=\left(\left(\mathcal{M} \circ \mathcal{N}^{\perp}\right) \cap\left(\mathcal{N}^{\perp} \circ \mathcal{M}\right)\right) \oplus\left(\left(\mathcal{M}^{\perp} \circ \mathcal{N}\right) \cap\left(\mathcal{N} \circ \mathcal{M}^{\perp}\right)\right) \\
& \oplus\left(\left(\mathcal{M}^{\perp} \circ \mathcal{N}^{\perp}\right) \cap\left(\mathcal{N}^{\perp} \circ \mathcal{M}^{\perp}\right)\right) \oplus\left(\left(\mathcal{M}^{\perp} \circ \mathcal{N}\right) \cap\left(\mathcal{M}^{\perp} \circ \mathcal{N}^{\perp}\right)\right) \\
& \oplus\left(\left(\mathcal{N}^{\perp} \circ \mathcal{M}\right) \cap\left(\mathcal{N}^{\perp} \circ \mathcal{M}^{\perp}\right)\right)
\end{aligned}
$$

(c) $\mathbb{C}^{m}$ can be decomposed as

$$
\begin{aligned}
& \mathbb{C}^{m}=((\mathcal{M} \circ \mathcal{N}) \cap(\mathcal{N} \circ \mathcal{M})) \oplus\left(\left(\mathcal{M} \circ \mathcal{N}^{\perp}\right) \cap\left(\mathcal{N}^{\perp} \circ \mathcal{M}\right)\right) \\
& \oplus\left(\left(\mathcal{M}^{\perp} \circ \mathcal{N}\right) \cap\left(\mathcal{N} \circ \mathcal{M}^{\perp}\right)\right) \oplus\left(\left(\mathcal{M}^{\perp} \circ \mathcal{N}^{\perp}\right) \cap\left(\mathcal{N}^{\perp} \circ \mathcal{M}^{\perp}\right)\right) \\
& \oplus\left((\mathcal{M} \circ \mathcal{N}) \cap\left(\mathcal{M} \circ \mathcal{N}^{\perp}\right)\right) \oplus\left((\mathcal{N} \circ \mathcal{M}) \cap\left(\mathcal{N} \circ \mathcal{M}^{\perp}\right)\right) \text {, } \\
& \mathbb{C}^{m}=((\mathcal{M} \circ \mathcal{N}) \cap(\mathcal{N} \circ \mathcal{M})) \oplus\left(\left(\mathcal{M} \circ \mathcal{N}^{\perp}\right) \cap\left(\mathcal{N}^{\perp} \circ \mathcal{M}\right)\right) \\
& \oplus\left(\left(\mathcal{M}^{\perp} \circ \mathcal{N}\right) \cap\left(\mathcal{N} \circ \mathcal{M}^{\perp}\right)\right) \oplus\left(\left(\mathcal{M}^{\perp} \circ \mathcal{N}^{\perp}\right) \cap\left(\mathcal{N}^{\perp} \circ \mathcal{M}^{\perp}\right)\right) \\
& \oplus\left((\mathcal{M} \circ \mathcal{N}) \cap\left(\mathcal{M} \circ \mathcal{N}^{\perp}\right)\right) \oplus\left(\left(\mathcal{N}^{\perp} \circ \mathcal{M}\right) \cap\left(\mathcal{N}^{\perp} \circ \mathcal{M}^{\perp}\right)\right) \text {, } \\
& \mathbb{C}^{m}=((\mathcal{M} \circ \mathcal{N}) \cap(\mathcal{N} \circ \mathcal{M})) \oplus\left(\left(\mathcal{M} \circ \mathcal{N}^{\perp}\right) \cap\left(\mathcal{N}^{\perp} \circ \mathcal{M}\right)\right) \\
& \oplus\left(\left(\mathcal{M}^{\perp} \circ \mathcal{N}\right) \cap\left(\mathcal{N} \circ \mathcal{M}^{\perp}\right)\right) \oplus\left(\left(\mathcal{M}^{\perp} \circ \mathcal{N}^{\perp}\right) \cap\left(\mathcal{N}^{\perp} \circ \mathcal{M}^{\perp}\right)\right) \\
& \oplus\left(\left(\mathcal{M}^{\perp} \circ \mathcal{N}\right) \cap\left(\mathcal{M}^{\perp} \circ \mathcal{N}^{\perp}\right)\right) \oplus\left((\mathcal{N} \circ \mathcal{M}) \cap\left(\mathcal{N} \circ \mathcal{M}^{\perp}\right)\right), \\
& \mathbb{C}^{m}=((\mathcal{M} \circ \mathcal{N}) \cap(\mathcal{N} \circ \mathcal{M})) \oplus\left(\left(\mathcal{M} \circ \mathcal{N}^{\perp}\right) \cap\left(\mathcal{N}^{\perp} \circ \mathcal{M}\right)\right) \\
& \oplus\left(\left(\mathcal{M}^{\perp} \circ \mathcal{N}\right) \cap\left(\mathcal{N} \circ \mathcal{M}^{\perp}\right)\right) \oplus\left(\left(\mathcal{M}^{\perp} \circ \mathcal{N}^{\perp}\right) \cap\left(\mathcal{N}^{\perp} \circ \mathcal{M}^{\perp}\right)\right) \\
& \oplus\left(\left(\mathcal{M}^{\perp} \circ \mathcal{N}\right) \cap\left(\mathcal{M}^{\perp} \circ \mathcal{N}^{\perp}\right)\right) \oplus\left(\left(\mathcal{N}^{\perp} \circ \mathcal{M}\right) \cap\left(\mathcal{N}^{\perp} \circ \mathcal{M}^{\perp}\right)\right) \text {. }
\end{aligned}
$$


Concerning relationship between two linear subspaces, the following two definitions are well known.

Definition 2.11. A pair of linear subspaces $\mathcal{M}$ and $\mathcal{N}$ in $\mathbb{C}^{m}$ are said to be in generic position if and only if the following four equalities hold

$$
\mathcal{M} \cap \mathcal{N}=\{0\}, \mathcal{M} \cap \mathcal{N}^{\perp}=\{0\}, \mathcal{M}^{\perp} \cap \mathcal{N}=\{0\}, \mathcal{M}^{\perp} \cap \mathcal{N}^{\perp}=\{0\} .
$$

Definition 2.12. A pair of linear subspaces $\mathcal{M}$ and $\mathcal{N}$ in $\mathbb{C}^{m}$ are said to be commutative if and only if

$$
\mathcal{M}=(\mathcal{M} \cap \mathcal{N}) \oplus\left(\mathcal{M} \cap \mathcal{N}^{\perp}\right) \text { and } / \text { or } \mathcal{N}=(\mathcal{M} \cap \mathcal{N}) \oplus\left(\mathcal{M}^{\perp} \cap \mathcal{N}\right) .
$$

The concept of generic position was introduced in [57], see also [79], while behaviors of two subspaces in generic position are well understood from all aspects; see, e.g., [3, 6, 25, 26, 42, 54, 55, 57, 66, 68, 71, 79, 80, $98,100,111,118,148]$. The concept of commuting subspaces was defined in [93, 112]. The JDSDIs in Theorems 2.5(a) and 2.6(a) enable us to sufficiently characterize behaviors of two subspaces and their algebraic operations, such as, disjoints of subspaces, commutativity of spaces, space inequalities and space identities are some simple relations between subspaces. In what follows, we collect some elementary or well-known results in the literature on disjoints of subspaces, commutativity of spaces, space inequalities and space identities, and present some new conclusions on these algebraic properties.

Corollary 2.13. Let $\mathcal{N}$ and $\mathcal{N}$ be two linear subspaces of $\mathbb{C}^{m}$. Then, the following statements are equivalent:

$\langle 1\rangle \mathcal{M} \cap \mathcal{N}=\{0\}$, namely, $\mathcal{M}$ and $\mathcal{N}$ are disjoint.

$\langle 2\rangle \mathcal{M}^{\perp}+\mathcal{N}^{\perp}=\mathbb{C}^{m}$.

$\langle 3\rangle \mathcal{M}+\mathcal{N}=\mathcal{M} \oplus \mathcal{N}$.

〈4〉 $\mathcal{M}=\mathcal{M} \circ \mathcal{N}^{\perp}$ and/or $\mathcal{N}=\mathcal{N} \circ \mathcal{M}^{\perp}$.

〈5) $\mathcal{M}+\mathcal{N}=\left(\mathcal{M} \circ \mathcal{N}^{\perp}\right) \oplus\left(\mathcal{N} \circ \mathcal{M}^{\perp}\right)$.

〈6) $\mathcal{M} \subseteq \mathcal{M}^{\perp}+\mathcal{N}^{\perp}$ and/or $\mathcal{N} \subseteq \mathcal{M}^{\perp}+\mathcal{N}^{\perp}$.

$\langle 7\rangle \mathcal{M}=\left(\mathcal{M} \cap \mathcal{N}^{\perp}\right) \oplus(\mathcal{M} \bullet \mathcal{N})$ and $/$ or $\mathcal{N}=\left(\mathcal{M}^{\perp} \cap \mathcal{N}\right) \oplus(\mathcal{N} \bullet \mathcal{M})$.

$\langle 8\rangle \mathcal{M}+\mathcal{N} \subseteq \mathcal{M}^{\perp}+\mathcal{N}^{\perp}$.

$\langle 9\rangle \mathcal{M}+\mathcal{N}=\left(\mathcal{M} \cap \mathcal{N}^{\perp}\right) \oplus\left(\mathcal{M}^{\perp} \cap \mathcal{N}\right) \oplus(\mathcal{M} \bullet \mathcal{N}) \oplus(\mathcal{N} \bullet \mathcal{M})$.

$\langle 10\rangle \mathcal{M}+\mathcal{N}^{\perp}=\left(\mathcal{M} \cap \mathcal{N}^{\perp}\right) \oplus\left(\mathcal{M}^{\perp} \cap \mathcal{N}^{\perp}\right) \oplus(\mathcal{M} \bullet \mathcal{N}) \oplus\left(\mathcal{N}^{\perp} \bullet \mathcal{M}\right)$.

$\langle 11\rangle \mathcal{M}^{\perp}+\mathcal{N}=\left(\mathcal{M}^{\perp} \cap \mathcal{N}\right) \oplus\left(\mathcal{M}^{\perp} \cap \mathcal{N}^{\perp}\right) \oplus\left(\mathcal{M}^{\perp} \bullet \mathcal{N}\right) \oplus(\mathcal{N} \bullet \mathcal{M})$.

$\langle 12\rangle \operatorname{Comm}(\mathcal{M}, \mathcal{N})=\left(\mathcal{M} \cap \mathcal{N}^{\perp}\right) \oplus\left(\mathcal{M}^{\perp} \cap \mathcal{N}\right) \oplus\left(\mathcal{M}^{\perp} \cap \mathcal{N}^{\perp}\right)$ with some/all of the four facts: $\mathbb{C}^{m}=\left(\mathcal{M} \cap \mathcal{N}^{\perp}\right) \oplus$ $\left(\mathcal{M}^{\perp} \cap \mathcal{N}\right) \oplus\left(\mathcal{M}^{\perp} \cap \mathcal{N}^{\perp}\right) \oplus(\mathcal{M} \bullet \mathcal{N}) \oplus(\mathcal{N} \bullet \mathcal{M}), \mathbb{C}^{m}=\left(\mathcal{M} \cap \mathcal{N}^{\perp}\right) \oplus\left(\mathcal{M}^{\perp} \cap \mathcal{N}\right) \oplus\left(\mathcal{M}^{\perp} \cap \mathcal{N}^{\perp}\right) \oplus(\mathcal{M} \bullet \mathcal{N}) \oplus\left(\mathcal{N}^{\perp} \bullet \mathcal{M}\right)$, $\mathbb{C}^{m}=\left(\mathcal{M} \cap \mathcal{N}^{\perp}\right) \oplus\left(\mathcal{M}^{\perp} \cap \mathcal{N}\right) \oplus\left(\mathcal{M}^{\perp} \cap \mathcal{N}^{\perp}\right) \oplus\left(\mathcal{M}^{\perp} \bullet \mathcal{N}\right) \oplus(\mathcal{N} \bullet \mathcal{M}), \mathbb{C}^{m}=\left(\mathcal{M} \cap \mathcal{N}^{\perp}\right) \oplus\left(\mathcal{M}^{\perp} \cap \mathcal{N}\right) \oplus\left(\mathcal{M}^{\perp} \cap\right.$ $\left.\mathcal{N}^{\perp}\right) \oplus\left(\mathcal{M}^{\perp} \bullet \mathcal{N}\right) \oplus\left(\mathcal{N}^{\perp} \bullet \mathcal{M}\right)$

$\langle 13\rangle P_{\mathcal{M}}=P_{\mathcal{M} \cap \mathcal{N}^{\perp}}+P_{\mathcal{M} \cdot \mathcal{N}}$ and $/$ or $P_{\mathcal{N}}=P_{\mathcal{M} \perp}^{\perp \cap \mathcal{N}}+P_{\mathcal{N}} \bullet \mathcal{M}$.

$\langle 14\rangle P_{\mathcal{M}} P_{\mathcal{N}}=P_{\mathcal{M} \cdot{ }_{N}} P_{\mathcal{N} \bullet \mathcal{N}}$ and/or $P_{\mathcal{N}} P_{\mathcal{M}}=P_{\mathcal{N} \bullet \mathcal{M}} P_{\mathcal{M}} \cdot \mathcal{N}$.

$\langle 15\rangle \operatorname{dim}(\mathcal{M}+\mathcal{N})=\operatorname{dim}(\mathcal{M})+\operatorname{dim}(\mathcal{N})$.

$\langle 16\rangle \operatorname{dim}\left(\mathcal{M}^{\perp}+\mathcal{N}^{\perp}\right)=m$.

$\langle 17\rangle \operatorname{dim}\left(\mathcal{N}^{\perp} \cap \mathcal{N}^{\perp}\right)=m-\operatorname{dim}(\mathcal{N})-\operatorname{dim}(\mathcal{N})$.

$\langle 18\rangle \operatorname{dim}(\mathcal{N})=\operatorname{dim}\left(\mathcal{N} \circ \mathcal{N}^{\perp}\right)$ and $/$ or $\operatorname{dim}(\mathcal{N})=\operatorname{dim}\left(\mathcal{N} \circ \mathcal{N}^{\perp}\right)$.

$\langle 19\rangle \operatorname{dim}(\mathcal{M}+\mathcal{N})=\operatorname{dim}\left(\mathcal{M} \circ \mathcal{N}^{\perp}\right)+\operatorname{dim}\left(\mathcal{N} \circ \mathcal{M}^{\perp}\right)$.

$\langle 20\rangle \operatorname{dim}(\mathcal{N})=\operatorname{dim}\left(\mathcal{M} \cap \mathcal{N}^{\perp}\right)+\operatorname{dim}(\mathcal{N} \bullet \mathcal{N})$ and $/$ or $\operatorname{dim}(\mathcal{N})=\operatorname{dim}\left(\mathcal{M}^{\perp} \cap \mathcal{N}\right)+\operatorname{dim}(\mathcal{N} \bullet \mathcal{N})$.

21) Some/all of the three facts: $\operatorname{dim}(\mathcal{M}+\mathcal{N})=\operatorname{dim}\left(\mathcal{M} \cap \mathcal{N}^{\perp}\right)+\operatorname{dim}\left(\mathcal{M}^{\perp} \cap \mathcal{N}\right)+\operatorname{dim}(\mathcal{N} \bullet \mathcal{N})+\operatorname{dim}(\mathcal{N} \bullet \mathcal{M})$ and some/all $\operatorname{dim}\left(\mathcal{M}+\mathcal{N}^{\perp}\right)=\operatorname{dim}\left(\mathcal{M} \cap \mathcal{N}^{\perp}\right)+\operatorname{dim}\left(\mathcal{M}^{\perp} \cap \mathcal{N}^{\perp}\right)+\operatorname{dim}(\mathcal{N} \bullet \mathcal{N})+\operatorname{dim}\left(\mathcal{N}^{\perp} \bullet \mathcal{M}\right), \operatorname{dim}\left(\mathcal{M}^{\perp}+\mathcal{N}\right)=$ $\operatorname{dim}\left(\mathcal{M}^{\perp} \cap \mathcal{N}\right)+\operatorname{dim}\left(\mathcal{M}^{\perp} \cap \mathcal{N}^{\perp}\right)+\operatorname{dim}\left(\mathcal{M}^{\perp} \bullet \mathcal{N}\right)+\operatorname{dim}(\mathcal{N} \bullet \mathcal{M})$

$\langle 22\rangle \operatorname{dim}(\mathcal{M}+\mathcal{N})=\operatorname{dim}\left(\mathcal{M}+\mathcal{N}^{\perp}\right)-\operatorname{dim}\left(\mathcal{M}^{\perp}+\mathcal{N}\right)+2 \operatorname{dim}(\mathcal{N})=\operatorname{dim}\left(\mathcal{M}^{\perp}+\mathcal{N}\right)-\operatorname{dim}\left(\mathcal{M}+\mathcal{N}^{\perp}\right)+2 \operatorname{dim}(\mathcal{N})$.

$\langle 23\rangle \operatorname{dim}(\mathcal{M}+\mathcal{N})=2 \operatorname{dim}(\mathcal{N})+\operatorname{dim}\left(\mathcal{M}^{\perp} \cap \mathcal{N}\right)-\operatorname{dim}\left(\mathcal{M} \cap \mathcal{N}^{\perp}\right)=2 \operatorname{dim}(\mathcal{N})+\operatorname{dim}\left(\mathcal{M} \cap \mathcal{N}^{\perp}\right)-\operatorname{dim}\left(\mathcal{M}^{\perp} \cap \mathcal{N}\right)$.

$\langle 24\rangle \operatorname{dim}\left(\mathcal{M} \cap \mathcal{N}^{\perp}\right)+\operatorname{dim}\left(\mathcal{M}^{\perp} \cap \mathcal{N}\right)+\operatorname{dim}\left(\mathcal{M}^{\perp} \cap \mathcal{N}^{\perp}\right)+\operatorname{dim}(\mathcal{M} \bullet \mathcal{N})+\operatorname{dim}(\mathcal{N} \bullet \mathcal{M})=m$. 
$\langle 25\rangle \operatorname{dim}\left(\mathcal{M} \cap \mathcal{N}^{\perp}\right)+\operatorname{dim}\left(\mathcal{M}^{\perp} \cap \mathcal{N}\right)+\operatorname{dim}\left(\mathcal{M}^{\perp} \cap \mathcal{N}^{\perp}\right)+\operatorname{dim}(\mathcal{M} \bullet \mathcal{N})+\operatorname{dim}\left(\mathcal{N}^{\perp} \bullet \mathcal{M}\right)=\operatorname{dim}\left(\mathcal{M} \cap \mathcal{N}^{\perp}\right)+\operatorname{dim}\left(\mathcal{M}^{\perp} \cap\right.$ $\mathcal{N})+\operatorname{dim}\left(\mathcal{M}^{\perp} \cap \mathcal{N}^{\perp}\right)+\operatorname{dim}\left(\mathcal{M}^{\perp} \bullet \mathcal{N}\right)+\operatorname{dim}(\mathcal{N} \bullet \mathcal{M})=\operatorname{dim}\left(\mathcal{M} \cap \mathcal{N}^{\perp}\right)+\operatorname{dim}\left(\mathcal{M}^{\perp} \cap \mathcal{N}\right)+\operatorname{dim}\left(\mathcal{M}^{\perp} \cap \mathcal{N}^{\perp}\right)+$ $\operatorname{dim}\left(\mathcal{M}^{\perp} \bullet \mathcal{N}\right)+\operatorname{dim}\left(\mathcal{N}^{\perp} \bullet \mathcal{N}\right)=m$.

Other three groups of equivalent statement can be established similarly for $\mathcal{M} \cap \mathcal{N}^{\perp}=\{0\}, \mathcal{M}^{\perp} \cap \mathcal{N}=\{0\}$, and $\mathcal{M}^{\perp} \cap \mathcal{N}^{\perp}=\{0\}$ to hold. Combining Theorem 2.13 with the three groups of equivalent statements will yield a family of identifying conditions for two subspaces to be in generic position. We shall present some of them in Theorem 4.2.

Corollary 2.14. Let $\mathcal{N}$ and $\mathcal{N}$ be two linear subspaces of $\mathbb{C}^{m}$. Then, the following statements are equivalent:

<1) $\mathcal{M}$ and $\mathcal{N}$ are commutative, i.e., $\mathcal{M}=(\mathcal{M} \cap \mathcal{N}) \oplus\left(\mathcal{M} \cap \mathcal{N}^{\perp}\right)$ and/or $\mathcal{N}=(\mathcal{M} \cap \mathcal{N}) \oplus\left(\mathcal{N}{ }^{\perp} \cap \mathcal{N}\right)$.

(2) $\mathcal{M}$ and $\mathcal{N}^{\perp}$ are commutative, i.e., $\mathcal{M}=(\mathcal{M} \cap \mathcal{N}) \oplus\left(\mathcal{M} \cap \mathcal{N}^{\perp}\right)$ and/or $\mathcal{N}^{\perp}=\left(\mathcal{M} \cap \mathcal{N}^{\perp}\right) \oplus\left(\mathcal{M} \mathcal{L}^{\perp} \cap \mathcal{N}^{\perp}\right)$.

33 $\mathcal{M}^{\perp}$ and $\mathcal{N}$ are commutative, i.e., $\mathcal{M}^{\perp}=\left(\mathcal{M}^{\perp} \cap \mathcal{N}\right) \oplus\left(\mathcal{M}^{\perp} \cap \mathcal{N}^{\perp}\right)$ and/or $\mathcal{N}=(\mathcal{M} \cap \mathcal{N}) \oplus\left(\mathcal{M}^{\perp} \cap \mathcal{N}\right)$.

〈4) $\mathcal{M}^{\perp}$ and $\mathcal{N}^{\perp}$ are commutative, i.e., $\mathcal{M}^{\perp}=\left(\mathcal{M}^{\perp} \cap \mathcal{N}\right) \oplus\left(\mathcal{M}^{\perp} \cap \mathcal{N}^{\perp}\right)$ and/or $\mathcal{N}^{\perp}=\left(\mathcal{M} \cap \mathcal{N}^{\perp}\right) \oplus\left(\mathcal{M}^{\perp} \cap \mathcal{N}^{\perp}\right)$.

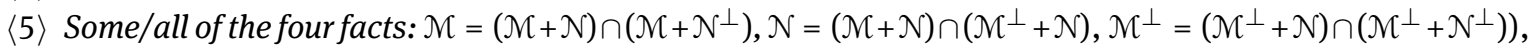
$\mathcal{N}^{\perp}=\left(\mathcal{M}+\mathcal{N}^{\perp}\right) \cap\left(\mathcal{M}^{\perp}+\mathcal{N}^{\perp}\right)$

〈6) Some/all of the eight facts: $\mathcal{M} \supseteq \mathcal{N} \circ \mathcal{M}$ and/or $\mathcal{M} \supseteq \mathcal{N}^{\perp} \circ \mathcal{M}, \mathcal{N} \supseteq \mathcal{M} \circ \mathcal{N}, \mathcal{N} \supseteq \mathcal{M}^{\perp} \circ \mathcal{N}, \mathcal{M}^{\perp} \supseteq \mathcal{N} \circ \mathcal{M}^{\perp}$, $\mathcal{M}^{\perp} \supseteq \mathcal{N}^{\perp} \circ \mathcal{M}^{\perp}, \mathcal{N}^{\perp} \supseteq \mathcal{M} \circ \mathcal{N}^{\perp}, \mathcal{N}^{\perp} \supseteq \mathcal{M}^{\perp} \circ \mathcal{N}^{\perp}$.

〈7) Some/all of the eight facts: $\mathcal{M} \subseteq(\mathcal{M} \cap \mathcal{N}) \oplus \mathcal{N}^{\perp}, \mathcal{M} \subseteq\left(\mathcal{M} \cap \mathcal{N}^{\perp}\right) \oplus \mathcal{N}, \mathcal{N} \subseteq(\mathcal{M} \cap \mathcal{N}) \oplus \mathcal{M} \mathcal{N}^{\perp}, \mathcal{N} \subseteq\left(\mathcal{M} \mathcal{K}^{\perp} \cap \mathcal{N}\right) \oplus \mathcal{M}$, $\mathcal{M}^{\perp} \subseteq\left(\mathcal{M}^{\perp} \cap \mathcal{N}\right) \oplus \mathcal{N}^{\perp}, \mathcal{M}^{\perp} \subseteq\left(\mathcal{M}^{\perp} \cap \mathcal{N}^{\perp}\right) \oplus \mathcal{N}, \mathcal{N}^{\perp} \subseteq\left(\mathcal{M} \cap \mathcal{N}^{\perp}\right) \oplus \mathcal{M}^{\perp}, \mathcal{N}^{\perp} \subseteq\left(\mathcal{M}^{\perp} \cap \mathcal{N}^{\perp}\right) \oplus \mathcal{M}$.

<8 Some/all of the four facts: $\mathcal{M} \cap \mathcal{N}=\mathcal{M} \circ \mathcal{N}=\mathcal{N} \circ \mathcal{M}, \mathcal{M} \cap \mathcal{N}^{\perp}=\mathcal{M} \circ \mathcal{N}^{\perp}=\mathcal{N}^{\perp} \circ \mathcal{M}, \mathcal{M}^{\perp} \cap \mathcal{N}=\mathcal{M}^{\perp} \circ \mathcal{N}=\mathcal{N} \circ \mathcal{M}^{\perp}$, $\mathcal{M}^{\perp} \cap \mathcal{N}^{\perp}=\mathcal{M}^{\perp} \circ \mathcal{N}^{\perp}=\mathcal{N}^{\perp} \circ \mathcal{M}^{\perp}$.

〈9) Some/all of the four facts: $\mathcal{M}+\mathcal{N}=\mathcal{M} \oplus\left(\mathcal{M}^{\perp} \cap \mathcal{N}\right)=\mathcal{N} \oplus\left(\mathcal{M} \cap \mathcal{N}^{\perp}\right), \mathcal{M}+\mathcal{N}^{\perp}=\mathcal{M} \oplus\left(\mathcal{M} \mathcal{N}^{\perp} \cap \mathcal{N}^{\perp}\right)=\mathcal{N}^{\perp} \oplus(\mathcal{M} \cap \mathcal{N})$, $\mathcal{M}^{\perp}+\mathcal{N}=\mathcal{M}^{\perp} \oplus(\mathcal{M} \cap \mathcal{N})=\mathcal{N} \oplus\left(\mathcal{M}^{\perp} \cap \mathcal{N}^{\perp}\right), \mathcal{M}^{\perp}+\mathcal{N}^{\perp}=\mathcal{M}^{\perp} \oplus\left(\mathcal{M} \cap \mathcal{N}^{\perp}\right)=\mathcal{N}^{\perp} \oplus\left(\mathcal{M} \mathcal{L}^{\perp} \cap \mathcal{N}\right)$.

〈10) Some/all of the four facts: $\mathcal{M} \circ \mathcal{N}=\mathcal{N} \circ \mathcal{M}, \mathcal{M} \circ \mathcal{N}^{\perp}=\mathcal{N}^{\perp} \circ \mathcal{M}, \mathcal{M}^{\perp} \circ \mathcal{N}=\mathcal{N} \circ \mathcal{M}^{\perp}, \mathcal{M}^{\perp} \circ \mathcal{N}^{\perp}=\mathcal{N}^{\perp} \circ \mathcal{M}^{\perp}$.

〈11) Some/all of the four facts: $\mathcal{M} \oplus\left(\mathcal{M}^{\perp} \cap \mathcal{N}\right)=\mathcal{N} \oplus\left(\mathcal{M} \cap \mathcal{N}^{\perp}\right)$ and/or $\mathcal{M} \oplus\left(\mathcal{M}^{\perp} \cap \mathcal{N}^{\perp}\right)=\mathcal{N}^{\perp} \oplus(\mathcal{M} \cap \mathcal{N})$, $\mathcal{M}^{\perp} \oplus\left(\mathcal{M} \cap \mathcal{N}^{\perp}\right)=\mathcal{N}^{\perp} \oplus\left(\mathcal{M}^{\perp} \cap \mathcal{N}\right), \mathcal{M}^{\perp} \oplus(\mathcal{M} \cap \mathcal{N})=\mathcal{N} \oplus\left(\mathcal{M}^{\perp} \cap \mathcal{N}^{\perp}\right)$.

<12〉 Some/all of the four facts: $\mathcal{M}+\mathcal{N}=(\mathcal{M} \cap \mathcal{N}) \oplus\left(\mathcal{M} \cap \mathcal{N}^{\perp}\right) \oplus\left(\mathcal{M}^{\perp} \cap \mathcal{N}\right), \mathcal{M}+\mathcal{N}^{\perp}=(\mathcal{M} \cap \mathcal{N}) \oplus\left(\mathcal{M} \cap \mathcal{N}^{\perp}\right) \oplus$ $\left(\mathcal{M}^{\perp} \cap \mathcal{N}^{\perp}\right), \mathcal{M}^{\perp}+\mathcal{N}=(\mathcal{M} \cap \mathcal{N}) \oplus\left(\mathcal{M}^{\perp} \cap \mathcal{N}\right) \oplus\left(\mathcal{M}^{\perp} \cap \mathcal{N}^{\perp}\right), \mathcal{M}^{\perp}+\mathcal{N}^{\perp}=\left(\mathcal{M} \cap \mathcal{N}^{\perp}\right) \oplus\left(\mathcal{M}^{\perp} \cap \mathcal{N}\right) \oplus\left(\mathcal{M}^{\perp} \cap \mathcal{N}^{\perp}\right)$.

$\langle 13\rangle \operatorname{Comm}(\mathcal{M}, \mathcal{N})=\mathbb{C}^{m}$.

<14) Some/all of the four facts: $\mathcal{M}^{\perp} \cap \mathcal{N}^{\perp}=\left(\mathcal{N}+\mathcal{N}^{\perp}\right) \cap\left(\mathcal{M}^{\perp}+\mathcal{N}\right) \cap\left(\mathcal{M}^{\perp}+\mathcal{N}^{\perp}\right)$ and/or $\mathcal{M}^{\perp} \cap \mathcal{N}=\left(\mathcal{M}^{\perp}+\mathcal{N}^{\perp}\right) \cap$ $\left(\mathcal{M}^{\perp}+\mathcal{N}\right) \cap(\mathcal{M}+\mathcal{N}), \mathcal{M} \cap \mathcal{N}^{\perp}=(\mathcal{M}+\mathcal{N}) \cap\left(\mathcal{M}+\mathcal{N}^{\perp}\right) \cap\left(\mathcal{M}^{\perp}+\mathcal{N}^{\perp}\right), \mathcal{M} \cap \mathcal{N}=(\mathcal{M}+\mathcal{N}) \cap\left(\mathcal{M}+\mathcal{N}^{\perp}\right) \cap\left(\mathcal{M}^{\perp}+\mathcal{N}\right)$.

$\langle 15\rangle$ Some/all of the four facts: $\mathcal{M} \bullet \mathcal{N}=(\mathcal{M} \circ \mathcal{N}) \cap\left(\mathcal{M} \circ \mathcal{N}^{\perp}\right)=\{0\}, \mathcal{N} \bullet \mathcal{M}=(\mathcal{N} \circ \mathcal{M}) \cap\left(\mathcal{N} \circ \mathcal{M}^{\perp}\right)=\{0\}$, $\mathcal{M}^{\perp} \bullet \mathcal{N}=\left(\mathcal{M}^{\perp} \circ \mathcal{N}\right) \cap\left(\mathcal{M}^{\perp} \circ \mathcal{N}^{\perp}\right)=\{0\}, \mathcal{N}^{\perp} \bullet \mathcal{M}=\left(\mathcal{N}^{\perp} \circ \mathcal{M}\right) \cap\left(\mathcal{N}^{\perp} \circ \mathcal{M}^{\perp}\right)=\{0\}$

<16) Some/all of the four facts: $\mathbb{C}^{m}=\mathcal{M} \oplus\left(\mathcal{M}^{\perp} \cap \mathcal{N}\right) \oplus\left(\mathcal{M}^{\perp} \cap \mathcal{N}^{\perp}\right), \mathbb{C}^{m}=\mathcal{N} \oplus\left(\mathcal{M} \cap \mathcal{N}^{\perp}\right) \oplus\left(\mathcal{M}^{\perp} \cap \mathcal{N}^{\perp}\right)$, $\mathbb{C}^{m}=\mathcal{M}^{\perp} \oplus(\mathcal{M} \cap \mathcal{N}) \oplus\left(\mathcal{M} \cap \mathcal{N}^{\perp}\right), \mathbb{C}^{m}=\mathcal{N}^{\perp} \oplus(\mathcal{M} \cap \mathcal{N}) \oplus\left(\mathcal{M}^{\perp} \cap \mathcal{N}\right)$.

$\langle 17\rangle$ Some/all of the four facts: $P_{\mathcal{M}}=P_{\mathcal{N} \cap \mathcal{N}}+P_{\mathcal{M} \cap \mathcal{N}^{\perp}}, P_{\mathcal{N}}=P_{\mathcal{M} \cap \mathcal{N}}+P_{\mathcal{M}^{\perp} \cap \mathcal{N}}, P_{\mathcal{M}^{\perp}}=P_{\mathcal{M}^{\perp} \cap \mathcal{N}}+P_{\mathcal{M}^{\perp} \cap \mathcal{N}^{\perp}}$, $P_{\mathcal{N}^{\perp}}=P_{\mathcal{M} \cap \mathcal{N}^{\perp}}+P_{\mathcal{M} \perp \cap \mathcal{N}^{\perp}}$.

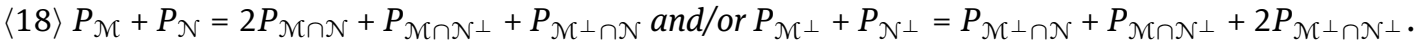

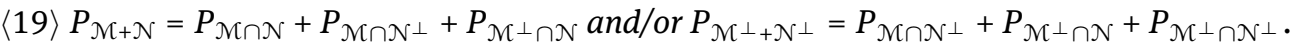

$\left\langle 20 I_{m}=P_{\mathcal{M} \cap \mathcal{N}}+P_{\mathcal{M} \cap \mathcal{N}^{\perp}}+P_{\mathcal{M}^{\perp} \cap \mathcal{N}}+P_{\mathcal{M M}^{\perp} \cap \mathcal{N}^{\perp}}\right.$.

21) Some/all of the four facts: $\operatorname{dim}(\mathcal{N})=\operatorname{dim}(\mathcal{M} \cap \mathcal{N})+\operatorname{dim}\left(\mathcal{M} \cap \mathcal{N}^{\perp}\right), \operatorname{dim}(\mathcal{N})=\operatorname{dim}(\mathcal{M} \cap \mathcal{N})+\operatorname{dim}\left(\mathcal{M}^{\perp} \cap \mathcal{N}\right)$, $\operatorname{dim}\left(\mathcal{M}^{\perp}\right)=\operatorname{dim}\left(\mathcal{M}^{\perp} \cap \mathcal{N}\right)+\operatorname{dim}\left(\mathcal{M}^{\perp} \cap \mathcal{N}^{\perp}\right), \operatorname{dim}\left(\mathcal{N}^{\perp}\right)=\operatorname{dim}\left(\mathcal{M} \cap \mathcal{N}^{\perp}\right)+\operatorname{dim}\left(\mathcal{M}^{\perp} \cap \mathcal{N}^{\perp}\right)$.

22 Some/all of the four facts: $\operatorname{dim}(\mathcal{N}+\mathcal{N})=\operatorname{dim}(\mathcal{N} \cap \mathcal{N})+\operatorname{dim}\left(\mathcal{N} \cap \mathcal{N}^{\perp}\right)+\operatorname{dim}\left(\mathcal{M}^{\perp} \cap \mathcal{N}\right), \operatorname{dim}\left(\mathcal{N}+\mathcal{N}^{\perp}\right)=$ $\operatorname{dim}(\mathcal{M} \cap \mathcal{N})+\operatorname{dim}\left(\mathcal{M} \cap \mathcal{N}^{\perp}\right)+\operatorname{dim}\left(\mathcal{M}^{\perp} \cap \mathcal{N}^{\perp}\right), \operatorname{dim}\left(\mathcal{M}^{\perp}+\mathcal{N}\right)=\operatorname{dim}(\mathcal{M} \cap \mathcal{N})+\operatorname{dim}\left(\mathcal{M}^{\perp} \cap \mathcal{N}\right)+\operatorname{dim}\left(\mathcal{M}^{\perp} \cap \mathcal{N}^{\perp}\right)$, $\operatorname{dim}\left(\mathcal{M}^{\perp}+\mathcal{N}^{\perp}\right)=\operatorname{dim}\left(\mathcal{M} \cap \mathcal{N}^{\perp}\right)+\operatorname{dim}\left(\mathcal{M}^{\perp} \cap \mathcal{N}\right)+\operatorname{dim}\left(\mathcal{M}^{\perp} \cap \mathcal{N}^{\perp}\right)$

23> Some/all of the four facts: $\operatorname{dim}(\mathcal{M} \cap \mathcal{N})=\operatorname{dim}(\mathcal{M} \circ \mathcal{N})=\operatorname{dim}(\mathcal{N} \circ \mathcal{M}), \operatorname{dim}\left(\mathcal{M} \cap \mathcal{N}^{\perp}\right)=\operatorname{dim}\left(\mathcal{M} \circ \mathcal{N}^{\perp}\right)=$ $\operatorname{dim}\left(\mathcal{N}^{\perp} \circ \mathcal{N}\right), \operatorname{dim}\left(\mathcal{M}^{\perp} \cap \mathcal{N}\right)=\operatorname{dim}\left(\mathcal{M}^{\perp} \circ \mathcal{N}\right)=\operatorname{dim}\left(\mathcal{N} \circ \mathcal{M}^{\perp}\right), \operatorname{dim}\left(\mathcal{M}^{\perp} \cap \mathcal{N}^{\perp}\right)=\operatorname{dim}\left(\mathcal{M}^{\perp} \circ \mathcal{N}^{\perp}\right)=$ $\operatorname{dim}\left(\mathcal{N}^{\perp} \circ \mathcal{M}^{\perp}\right)$. 
24) Some/all of the four facts: $\operatorname{dim}(\mathcal{M}+\mathcal{N})=\operatorname{dim}(\mathcal{M})+\operatorname{dim}\left(\mathcal{M}^{\perp} \cap \mathcal{N}\right)=\operatorname{dim}(\mathcal{N})+\operatorname{dim}\left(\mathcal{M} \cap \mathcal{N}^{\perp}\right), \operatorname{dim}(\mathcal{M}+$ $\left.\mathcal{N}^{\perp}\right)=\operatorname{dim}(\mathcal{M})+\operatorname{dim}\left(\mathcal{M}^{\perp} \cap \mathcal{N}^{\perp}\right)=\operatorname{dim}\left(\mathcal{N}^{\perp}\right)+\operatorname{dim}(\mathcal{M} \cap \mathcal{N}), \operatorname{dim}\left(\mathcal{M}^{\perp}+\mathcal{N}\right)=\operatorname{dim}\left(\mathcal{M}^{\perp}\right)+\operatorname{dim}(\mathcal{M} \cap \mathcal{N})=$ $\operatorname{dim}(\mathcal{N})+\operatorname{dim}\left(\mathcal{M}^{\perp} \cap \mathcal{N}^{\perp}\right), \operatorname{dim}\left(\mathcal{M}^{\perp}+\mathcal{N}^{\perp}\right)=\operatorname{dim}\left(\mathcal{M}^{\perp}\right)+\operatorname{dim}\left(\mathcal{M} \cap \mathcal{N}^{\perp}\right)=\operatorname{dim}\left(\mathcal{N}^{\perp}\right)+\operatorname{dim}\left(\mathcal{M}^{\perp} \cap \mathcal{N}\right)$.

More equivalent statements for two subspaces to be commutative will be given in Theorem 5.1.

Corollary 2.15. Let $\mathcal{N}$ and $\mathcal{N}$ be two linear subspaces of $\mathbb{C}^{m}$. Then, the following statements are equivalent:

$\langle 1\rangle \mathcal{M} \supseteq \mathcal{N}$ and/or $\mathcal{M}^{\perp} \subseteq \mathcal{N}^{\perp}$.

(2) $\mathcal{M}+\mathcal{N}=\mathcal{M}$ and/or $\mathcal{M}^{\perp} \cap \mathcal{N}^{\perp}=\mathcal{M}^{\perp}$.

$\langle 3\rangle \mathcal{M}^{\perp}+\mathcal{N}^{\perp}=\mathcal{N}^{\perp}$ and/or $\mathcal{M} \cap \mathcal{N}=\mathcal{N}$.

〈4) $\mathcal{M}=\left(\mathcal{M} \cap \mathcal{N}^{\perp}\right) \oplus \mathcal{N}$ and/or $\mathcal{M}^{\perp}=\left(\mathcal{M}^{\perp}+\mathcal{N}\right) \cap \mathcal{N}^{\perp}$.

$\langle 5\rangle \mathcal{N}^{\perp}=\mathcal{M}^{\perp} \oplus\left(\mathcal{M} \cap \mathcal{N}^{\perp}\right)$ and/or $\mathcal{N}=\mathcal{M} \cap\left(\mathcal{M}^{\perp}+\mathcal{N}\right)$.

$\langle 6\rangle(\mathcal{M} \cap \mathcal{N}) \oplus\left(\mathcal{M} \cap \mathcal{N}^{\perp}\right) \oplus\left(\mathcal{M}^{\perp} \cap \mathcal{N}^{\perp}\right)=\mathbb{C}^{m}$ and/or $(\mathcal{M}+\mathcal{N}) \cap\left(\mathcal{M}^{\perp}+\mathcal{N}\right) \cap\left(\mathcal{M}^{\perp}+\mathcal{N}^{\perp}\right)=\{0\}$.

$\langle 7\rangle \operatorname{dim}(\mathcal{M}+\mathcal{N})=\operatorname{dim}(\mathcal{N})$ and/or $\operatorname{dim}\left(\mathcal{M}^{\perp}+\mathcal{N}^{\perp}\right)=\operatorname{dim}\left(\mathcal{N}^{\perp}\right)$.

$\langle 8\rangle \operatorname{dim}(\mathcal{M} \cap \mathcal{N})=\operatorname{dim}(\mathcal{N})$ and $/$ or $\operatorname{dim}\left(\mathcal{M}^{\perp} \cap \mathcal{N}^{\perp}\right)=\operatorname{dim}\left(\mathcal{M}^{\perp}\right)$.

$\langle 9\rangle \operatorname{dim}(\mathcal{M} \cap \mathcal{N})+\operatorname{dim}\left(\mathcal{M} \cap \mathcal{N}^{\perp}\right)+\operatorname{dim}\left(\mathcal{M}^{\perp} \cap \mathcal{N}^{\perp}\right)=m$.

$\langle 10\rangle \mathcal{M}^{\perp} \cap \mathcal{N}=\{0\}$ with $\mathcal{M}$ and $\mathcal{N}$ being commutative.

$\langle 11\rangle \mathcal{M}^{\perp} \cap \mathcal{N}=\{0\}$ with one/both of the two facts: $\mathcal{N}=(\mathcal{M}+\mathcal{N}) \cap\left(\mathcal{M}^{\perp}+\mathcal{N}\right), \mathcal{M}^{\perp}=\left(\mathcal{M}^{\perp}+\mathcal{N}\right) \cap\left(\mathcal{M}^{\perp}+\mathcal{N}^{\perp}\right)$.

$\langle 12\rangle \mathcal{M}^{\perp} \cap \mathcal{N}=\{0\}$ with some/all of the seven facts: $\mathcal{M} \supseteq \mathcal{N}^{\perp} \circ \mathcal{M}, \mathcal{N} \supseteq \mathcal{M} \circ \mathcal{N}, \mathcal{N} \supseteq \mathcal{M}^{\perp} \circ \mathcal{N}, \mathcal{M}^{\perp} \supseteq \mathcal{N} \circ \mathcal{M}^{\perp}$, $\mathcal{M}^{\perp} \supseteq \mathcal{N}^{\perp} \circ \mathcal{M}^{\perp}, \mathcal{N}^{\perp} \supseteq \mathcal{M} \circ \mathcal{N}^{\perp}, \mathcal{N}^{\perp} \supseteq \mathcal{M}^{\perp} \circ \mathcal{N}^{\perp}$.

$\langle 13\rangle \mathcal{M}^{\perp} \cap \mathcal{N}=\{0\}$ with some/all of the six facts: $\mathcal{M} \subseteq(\mathcal{M} \cap \mathcal{N}) \oplus \mathcal{N}^{\perp}, \mathcal{M} \subseteq\left(\mathcal{M} \cap \mathcal{N}^{\perp}\right) \oplus \mathcal{N}, \mathcal{N} \subseteq(\mathcal{M} \cap \mathcal{N}) \oplus \mathcal{M}^{\perp}$, $\mathcal{M}^{\perp} \subseteq\left(\mathcal{M}^{\perp} \cap \mathcal{N}^{\perp}\right) \oplus \mathcal{N}, \mathcal{N}^{\perp} \subseteq\left(\mathcal{M} \cap \mathcal{N}^{\perp}\right) \oplus \mathcal{M}^{\perp}, \mathcal{N}^{\perp} \subseteq\left(\mathcal{M}^{\perp} \cap \mathcal{N}^{\perp}\right) \oplus \mathcal{M}$.

$\langle 14\rangle \mathcal{M}^{\perp} \cap \mathcal{N}=\{0\}$ with some/all of the three facts: $\mathcal{M} \circ \mathcal{N}=\mathcal{M} \cap \mathcal{N}, \mathcal{M} \circ \mathcal{N}^{\perp}=\mathcal{M} \cap \mathcal{N}^{\perp}, \mathcal{N}^{\perp} \circ \mathcal{M}=\mathcal{M} \cap \mathcal{N}^{\perp}$.

$\langle 15\rangle \mathcal{M}^{\perp} \circ \mathcal{N}=\{0\}$ and/or $\mathcal{N} \circ \mathcal{M}^{\perp}=\{0\}$.

$\langle 16\rangle \mathcal{M}^{\perp} \cap \mathcal{N}=\{0\}$ with one/both of the two facts: $\mathcal{M}^{\perp} \circ \mathcal{N}^{\perp}=\mathcal{M}^{\perp} \cap \mathcal{N}^{\perp}, \mathcal{N}^{\perp} \circ \mathcal{M}^{\perp}=\mathcal{M}^{\perp} \cap \mathcal{N}^{\perp}$.

$\langle 17\rangle \mathcal{M}^{\perp} \cap \mathcal{N}=\{0\}$ and $\mathcal{N}+\mathcal{N}=\mathcal{N} \oplus\left(\mathcal{M} \cap \mathcal{N}^{\perp}\right)$.

$\langle 18\rangle \mathcal{M} \oplus\left(\mathcal{M}^{\perp} \cap \mathcal{N}^{\perp}\right)=\mathbb{C}^{m}$ and/or $\mathcal{N}^{\perp} \oplus(\mathcal{M} \cap \mathcal{N})=\mathbb{C}^{m}$.

$\langle 19\rangle \mathcal{M}^{\perp} \cap \mathcal{N}=\{0\}$ with one/both of the two facts: $\mathcal{M}^{\perp} \oplus \mathcal{N}=\mathcal{M}^{\perp} \oplus(\mathcal{M} \cap \mathcal{N}), \mathcal{M}^{\perp} \oplus \mathcal{N}=\mathcal{N} \oplus\left(\mathcal{M}^{\perp} \cap \mathcal{N}^{\perp}\right)$.

$\langle 20\rangle \mathcal{M}^{\perp} \cap \mathcal{N}=\{0\}$ and $\mathcal{M}^{\perp}+\mathcal{N}^{\perp}=\mathcal{M}^{\perp} \oplus\left(\mathcal{M} \cap \mathcal{N}^{\perp}\right)$.

$\langle 21\rangle \mathcal{M}^{\perp} \cap \mathcal{N}=\{0\}$ with one/both of the two facts: $\mathcal{M} \circ \mathcal{N}=\mathcal{N}, \mathcal{M}^{\perp} \circ \mathcal{N}^{\perp}=\mathcal{M}^{\perp}$.

$\langle 22\rangle \mathcal{M}^{\perp} \cap \mathcal{N}=\{0\}$ with one/both of the two facts: $\mathcal{M} \circ \mathcal{N}^{\perp}=\mathcal{N}^{\perp} \circ \mathcal{M}, \mathcal{M}^{\perp} \circ \mathcal{N}=\mathcal{N} \circ \mathcal{M}^{\perp}$.

$\langle 23\rangle \mathcal{M}^{\perp} \cap \mathcal{N}=\{0\}$ with one/both of the two facts: $\mathcal{M} \oplus\left(\mathcal{M}^{\perp} \cap \mathcal{N}^{\perp}\right)=\mathcal{N}^{\perp} \oplus(\mathcal{M} \cap \mathcal{N}), \mathcal{M}^{\perp} \oplus(\mathcal{M} \cap \mathcal{N})=$ $\mathcal{N} \oplus\left(\mathcal{M}^{\perp} \cap \mathcal{N}^{\perp}\right)$.

(24) $\mathcal{M}^{\perp} \cap \mathcal{N}=\{0\}$ with some/all of the three facts: $\mathcal{M}+\mathcal{N}=(\mathcal{M} \cap \mathcal{N}) \oplus\left(\mathcal{M} \cap \mathcal{N}^{\perp}\right), \mathcal{M} \mathcal{L}^{\perp}+\mathcal{N}=(\mathcal{M} \cap \mathcal{N}) \oplus\left(\mathcal{M}^{\perp} \cap \mathcal{N}^{\perp}\right)$, $\mathcal{M}^{\perp}+\mathcal{N}^{\perp}=\left(\mathcal{M} \cap \mathcal{N}^{\perp}\right) \oplus\left(\mathcal{M}^{\perp} \cap \mathcal{N}^{\perp}\right)$.

$\langle 25\rangle \mathcal{M}^{\perp} \cap \mathcal{N}=\{0\}$ with one/both of the two facts: $\mathcal{M}^{\perp} \cap \mathcal{N}^{\perp}=\left(\mathcal{M}^{\perp}+\mathcal{N}\right) \cap\left(\mathcal{M}^{\perp}+\mathcal{N}^{\perp}\right), \mathcal{M} \cap \mathcal{N}^{\perp}=(\mathcal{M}+\mathcal{N}) \cap$ $\left(\mathcal{M}^{\perp}+\mathcal{N}^{\perp}\right), \mathcal{M} \cap \mathcal{N}=(\mathcal{M}+\mathcal{N}) \cap\left(\mathcal{M}^{\perp}+\mathcal{N}\right)$.

$\langle 26\rangle \mathcal{M}^{\perp} \cap \mathcal{N}=\{0\}$ with one/both of the two facts: $\mathcal{N} \bullet \mathcal{N}=\{0\}, \mathcal{N}^{\perp} \bullet \mathcal{M}=\{0\}$.

$\langle 27\rangle \mathcal{M}^{\perp} \cap \mathcal{N}=\{0\}$ with one/both of the two facts: $\mathcal{M}^{\perp}+(\mathcal{M} \cap \mathcal{N})+\left(\mathcal{M} \cap \mathcal{N}^{\perp}\right)=\mathbb{C}^{m}, \mathcal{N}+\left(\mathcal{M} \cap \mathcal{N}^{\perp}\right)+\left(\mathcal{M}^{\perp} \cap \mathcal{N}^{\perp}\right)=$ $\mathbb{C}^{m}$.

$\langle 28\rangle \mathcal{M}^{\perp} \cap \mathcal{N}=\{0\}$ with one/both of the two facts: $P_{\mathcal{M}}=P_{\mathcal{M} \cap \mathcal{N}}+P_{\mathcal{M} \cap \mathcal{N}^{\perp}}, P_{\mathcal{N}^{\perp}}=P_{\mathcal{M} \cap \mathcal{N}^{\perp}}+P_{\mathcal{M}^{\perp} \cap \mathcal{N}^{\perp}}$.

$\langle 29\rangle \mathcal{M}^{\perp} \cap \mathcal{N}=\{0\}$ with one/both of the two facts: $P_{\mathcal{N}}=P_{\mathcal{M} \cap \mathcal{N}}, P_{\mathcal{M}^{\perp}}=P_{\mathcal{M}^{\perp} \cap \mathcal{N}^{\perp}}$.

$\langle 30\rangle \mathcal{M}^{\perp} \cap \mathcal{N}=\{0\}$ with one/both of the two facts: $P_{\mathcal{M}}+P_{\mathcal{N}}=2 P_{\mathcal{M} \cap \mathcal{N}}+P_{\mathcal{M} \cap \mathcal{N}^{\perp}}, P_{\mathcal{M}^{\perp}}+P_{\mathcal{N}^{\perp}}=P_{\mathcal{M}^{\prime} \cap \mathcal{N}^{\perp}}+$ $2 P_{\mathcal{M}^{\perp} \cap \mathcal{N}^{\perp}}$.

$\langle 31\rangle \mathcal{M}^{\perp} \cap \mathcal{N}=\{0\}$ with one/both of the two facts: $P_{\mathcal{M}+\mathcal{N}}=P_{\mathcal{M} \cap \mathcal{N}}+P_{\mathcal{M} \cap \mathcal{N}^{\perp}}, P_{\mathcal{M}^{\perp}+\mathcal{N}^{\perp}}=P_{\mathcal{M} \cap \mathcal{N}^{\perp}}+P_{\mathcal{M}^{\perp} \cap \mathcal{N}^{\perp}}$.

$\langle 32\rangle \mathcal{M}^{\perp} \cap \mathcal{N}=\{0\}$ and $I_{m}=P_{\mathcal{M} \cap \mathcal{N}}+P_{{\mathcal{M} \cap \mathcal{N}^{\perp}}+P_{\mathcal{M}}{ }^{\perp} \cap \mathcal{N}^{\perp}}$.

$\langle 33\rangle \mathcal{M}^{\perp} \cap \mathcal{N}=\{0\}$ with one/both of the two facts: $\operatorname{dim}(\mathcal{M})=\operatorname{dim}(\mathcal{M} \cap \mathcal{N})+\operatorname{dim}\left(\mathcal{M} \cap \mathcal{N}^{\perp}\right), \operatorname{dim}\left(\mathcal{N}^{\perp}\right)=$ $\operatorname{dim}\left(\mathcal{M} \cap \mathcal{N}^{\perp}\right)+\operatorname{dim}\left(\mathcal{M}^{\perp} \cap \mathcal{N}^{\perp}\right)$.

〈34〉 $\mathcal{M}^{\perp} \cap \mathcal{N}=\{0\}$ with some/all of the three facts: $\operatorname{dim}(\mathcal{N}+\mathcal{N})=\operatorname{dim}(\mathcal{M} \cap \mathcal{N})+\operatorname{dim}\left(\mathcal{N} \cap \mathcal{N}^{\perp}\right), \operatorname{dim}\left(\mathcal{M}^{\perp}+\mathcal{N}\right)=$ $\operatorname{dim}(\mathcal{M} \cap \mathcal{N})+\operatorname{dim}\left(\mathcal{M}^{\perp} \cap \mathcal{N}^{\perp}\right), \operatorname{dim}\left(\mathcal{M}^{\perp}+\mathcal{N}^{\perp}\right)=\operatorname{dim}\left(\mathcal{M} \cap \mathcal{N}^{\perp}\right)+\operatorname{dim}\left(\mathcal{M}^{\perp} \cap \mathcal{N}^{\perp}\right)$.

〈35〉 $\mathcal{M}^{\perp} \cap \mathcal{N}=\{0\}$ and $\operatorname{dim}\left(\mathcal{M}+\mathcal{N}^{\perp}\right)=\operatorname{dim}(\mathcal{M} \cap \mathcal{N})+\operatorname{dim}\left(\mathcal{M} \cap \mathcal{N}^{\perp}\right)+\operatorname{dim}\left(\mathcal{M}^{\perp} \cap \mathcal{N}^{\perp}\right)$. 
36) $\mathcal{M}^{\perp} \cap \mathcal{N}=\{0\}$ with some/all of the five facts: $\operatorname{dim}(\mathcal{M} \circ \mathcal{N})=\operatorname{dim}(\mathcal{M} \cap \mathcal{N}), \operatorname{dim}\left(\mathcal{M} \circ \mathcal{N}^{\perp}\right)=\operatorname{dim}\left(\mathcal{M} \cap \mathcal{N}^{\perp}\right)$, $\operatorname{dim}\left(\mathcal{N}^{\perp} \circ \mathcal{M}\right)=\operatorname{dim}\left(\mathcal{N} \cap \mathcal{N}^{\perp}\right), \operatorname{dim}\left(\mathcal{M}^{\perp} \circ \mathcal{N}^{\perp}\right)=\operatorname{dim}\left(\mathcal{M}^{\perp} \cap \mathcal{N}^{\perp}\right), \operatorname{dim}\left(\mathcal{N}^{\perp} \circ \mathcal{M}^{\perp}\right)=\operatorname{dim}\left(\mathcal{M}^{\perp} \cap \mathcal{N}^{\perp}\right)$.

$\langle 37\rangle \mathcal{M}^{\perp} \cap \mathcal{N}=\{0\}$ with some/all of the four facts: $\operatorname{dim}(\mathcal{M}+\mathcal{N})=\operatorname{dim}(\mathcal{N})+\operatorname{dim}\left(\mathcal{M} \cap \mathcal{N}^{\perp}\right), \operatorname{dim}\left(\mathcal{M}^{\perp}+\mathcal{N}\right)=$ $\operatorname{dim}\left(\mathcal{M}^{\perp}\right)+\operatorname{dim}(\mathcal{M} \cap \mathcal{N}), \operatorname{dim}\left(\mathcal{M}^{\perp}+\mathcal{N}\right)=\operatorname{dim}(\mathcal{N})+\operatorname{dim}\left(\mathcal{M}^{\perp} \cap \mathcal{N}^{\perp}\right), \operatorname{dim}\left(\mathcal{M}^{\perp}+\mathcal{N}^{\perp}\right)=\operatorname{dim}\left(\mathcal{M}^{\perp}\right)+\operatorname{dim}(\mathcal{M} \cap$ $\left.\mathcal{N}^{\perp}\right)$.

More equivalent statements for two subspaces to satisfy inequalities will be given in Theorem 6.1.

Corollary 2.16. Let $\mathcal{N}$ and $\mathcal{N}$ be two linear subspaces of $\mathbb{C}^{m}$. Then, the following statements are equivalent:

$\langle 1\rangle \mathcal{M}=\mathcal{N}$, i.e., both $\mathcal{M} \supseteq \mathcal{N}$ and $\mathcal{M} \subseteq \mathcal{N}$.

(2) $\mathcal{M}^{\perp}=\mathcal{N}^{\perp}$, i.e., both $\mathcal{M}^{\perp} \supseteq \mathcal{N}^{\perp}$ and $\mathcal{M}^{\perp} \subseteq \mathcal{N}^{\perp}$.

33 $\{\mathcal{M} \subseteq \mathcal{N}$ and $\operatorname{dim}(\mathcal{N})=\operatorname{dim}(\mathcal{N})\}$ and/or $\{\mathcal{N} \supseteq \mathcal{N}$ and $\operatorname{dim}(\mathcal{N})=\operatorname{dim}(\mathcal{N})\}$.

44 $\left\{\mathcal{M}^{\perp} \subseteq \mathcal{N}^{\perp}\right.$ and $\left.\operatorname{dim}\left(\mathcal{M}^{\perp}\right)=\operatorname{dim}\left(\mathcal{N}^{\perp}\right)\right\}$ and/or $\left\{\mathcal{M}^{\perp} \supseteq \mathcal{N}^{\perp}\right.$ and $\left.\operatorname{dim}\left(\mathcal{M} \mathcal{M}^{\perp}\right)=\operatorname{dim}\left(\mathcal{N}^{\perp}\right)\right\}$.

〈5) $\mathcal{M}+\mathcal{N}=\mathcal{M} \cap \mathcal{N}$ and/or $\mathcal{M}^{\perp}+\mathcal{N}^{\perp}=\mathcal{M}^{\perp} \cap \mathcal{N}^{\perp}$.

66) $\left\{\mathcal{M}=\mathcal{N} \oplus\left(\mathcal{M} \cap \mathcal{N}^{\perp}\right)\right.$ and $\left.\mathcal{N}=\mathcal{M} \oplus\left(\mathcal{M}^{\perp} \cap \mathcal{N}\right)\right\}$ and/or $\left\{\mathcal{M}^{\perp}=\mathcal{N}^{\perp} \oplus\left(\mathcal{M} \mathcal{L}^{\perp} \cap \mathcal{N}\right)\right.$ and $\left.\mathcal{N}^{\perp}=\mathcal{M}^{\perp} \oplus\left(\mathcal{M} \cap \mathcal{N}^{\perp}\right)\right\}$.

〈7) $\left\{\mathcal{M}=\mathcal{N} \cap\left(\mathcal{M}+\mathcal{N}^{\perp}\right)\right.$ and $\left.\mathcal{N}=\mathcal{M} \cap\left(\mathcal{M}^{\perp}+\mathcal{N}\right)\right\}$ and/or $\left\{\mathcal{M}^{\perp}=\mathcal{N}^{\perp} \cap\left(\mathcal{M}^{\perp}+\mathcal{N}\right)\right.$ and $\left.\mathcal{N}^{\perp}=\mathcal{M}^{\perp} \cap\left(\mathcal{M}+\mathcal{N}^{\perp}\right)\right\}$.

$\langle 8\rangle(\mathcal{M} \cap \mathcal{N}) \oplus\left(\mathcal{M}^{\perp} \cap \mathcal{N}^{\perp}\right)=\mathbb{C}^{m}$ and/or $(\mathcal{M}+\mathcal{N}) \cap\left(\mathcal{M}^{\perp}+\mathcal{N}^{\perp}\right)=\{0\}$.

〈9) $2 \operatorname{dim}(\mathcal{M}+\mathcal{N})=\operatorname{dim}(\mathcal{N})+\operatorname{dim}(\mathcal{N})$ and $/$ or $2 \operatorname{dim}\left(\mathcal{M}^{\perp}+\mathcal{N}^{\perp}\right)=\operatorname{dim}\left(\mathcal{M}^{\perp}\right)+\operatorname{dim}\left(\mathcal{N}^{\perp}\right)$.

$\langle 10\rangle \operatorname{dim}(\mathcal{M}+\mathcal{N})=\operatorname{dim}(\mathcal{N})=\operatorname{dim}(\mathcal{N})$ and $/$ or $\operatorname{dim}\left(\mathcal{M}^{\perp}+\mathcal{N}^{\perp}\right)=\operatorname{dim}\left(\mathcal{M}^{\perp}\right)=\operatorname{dim}\left(\mathcal{N}^{\perp}\right)$.

$\langle 11\rangle \operatorname{dim}(\mathcal{N}+\mathcal{N})=\operatorname{dim}(\mathcal{M} \cap \mathcal{N})$ and $/$ or $\operatorname{dim}\left(\mathcal{M}^{\perp}+\mathcal{N}^{\perp}\right)=\operatorname{dim}\left(\mathcal{M}^{\perp} \cap \mathcal{N}^{\perp}\right)$.

$\langle 12\rangle \operatorname{dim}(\mathcal{M} \cap \mathcal{N})=\operatorname{dim}(\mathcal{N})=\operatorname{dim}(\mathcal{N})$ and $/$ or $\operatorname{dim}\left(\mathcal{M}^{\perp} \cap \mathcal{N}^{\perp}\right)=\operatorname{dim}\left(\mathcal{M}^{\perp}\right)=\operatorname{dim}\left(\mathcal{N}^{\perp}\right)$.

$\langle 13\rangle \operatorname{dim}(\mathcal{M} \cap \mathcal{N})+\operatorname{dim}\left(\mathcal{M}^{\perp} \cap \mathcal{N}^{\perp}\right)=m$.

$\langle 14\rangle \operatorname{dim}(\mathcal{M}+\mathcal{N})+\operatorname{dim}\left(\mathcal{M}^{\perp}+\mathcal{N}^{\perp}\right)=m$.

(15) Some/all of the four facts: $\left\{\mathcal{M} \cap \mathcal{N}^{\perp}=\{0\}\right.$ and $\left.\mathcal{M} \supseteq \mathcal{N}\right\},\left\{\mathcal{M}{ }^{\perp} \cap \mathcal{N}=\{0\}\right.$ and $\left.\mathcal{M} \subseteq \mathcal{N}\right\},\left\{\mathcal{M} \cap \mathcal{N}^{\perp}=\{0\}\right.$ and $\left.\mathcal{M}^{\perp} \subseteq \mathcal{N}^{\perp}\right\},\left\{\mathcal{M}^{\perp} \cap \mathcal{N}=\{0\}\right.$ and $\left.\mathcal{M}^{\perp} \supseteq \mathcal{N}^{\perp}\right\}$

$\langle 16\rangle \mathcal{M} \cap \mathcal{N}^{\perp}=\mathcal{M}^{\perp} \cap \mathcal{N}=\{0\}$, and $\mathcal{M}$ and $\mathcal{N}$ are commutative.

$\langle 17\rangle \mathcal{M} \cap \mathcal{N}^{\perp}=\mathcal{M}^{\perp} \cap \mathcal{N}=\{0\}$ with some/all of the four facts: $\mathcal{M}=\mathcal{M} \cap \mathcal{N}, \mathcal{N}=\mathcal{M} \cap \mathcal{N}, \mathcal{M}^{\perp}=\mathcal{M}^{\perp} \cap \mathcal{N}^{\perp}$, $\mathcal{N}^{\perp}=\mathcal{M}^{\perp} \cap \mathcal{N}^{\perp}$

$\langle 18\rangle \mathcal{M} \cap \mathcal{N}^{\perp}=\mathcal{M}^{\perp} \cap \mathcal{N}=\{0\}$ with some/all of the four facts: $\mathcal{M}=\mathcal{M}+\mathcal{N}, \mathcal{N}=\mathcal{M}+\mathcal{N}, \mathcal{M}^{\perp}=\mathcal{M}^{\perp}+\mathcal{N}^{\perp}$, $\mathcal{N}^{\perp}=\mathcal{M}^{\perp}+\mathcal{N}^{\perp}$

$\langle 19\rangle \mathcal{M} \cap \mathcal{N}^{\perp}=\mathcal{M}^{\perp} \cap \mathcal{N}=\{0\}$ with some/all of the four facts: $\mathcal{M} \supseteq \mathcal{N}, \mathcal{N} \supseteq \mathcal{M}, \mathcal{M}^{\perp} \supseteq \mathcal{N}^{\perp}, \mathcal{N}^{\perp} \supseteq \mathcal{M}^{\perp}$

$\langle 20\rangle \mathcal{M} \cap \mathcal{N}^{\perp}=\mathcal{M}^{\perp} \cap \mathcal{N}=\{0\}$ with some/all of the four facts: $\mathcal{M} \circ \mathcal{N}^{\perp}=\{0\}, \mathcal{N} \circ \mathcal{M}^{\perp}=\{0\}, \mathcal{M}^{\perp} \circ \mathcal{N}=\{0\}$, $\mathcal{N}^{\perp} \circ \mathcal{M}=\{0\}$.

$\langle 21\rangle \mathcal{M} \cap \mathcal{N}^{\perp}=\mathcal{M}^{\perp} \cap \mathcal{N}=\{0\}$ with some/all of the four facts: $\mathcal{M} \oplus\left(\mathcal{M}^{\perp} \cap \mathcal{N}^{\perp}\right)=\mathbb{C}^{m}, \mathcal{N} \oplus\left(\mathcal{M}^{\perp} \cap \mathcal{N}^{\perp}\right)=\mathbb{C}^{m}$, $\mathcal{M}^{\perp} \oplus(\mathcal{M} \cap \mathcal{N})=\mathbb{C}^{m}, \mathcal{N}^{\perp} \oplus(\mathcal{M} \cap \mathcal{N})=\mathbb{C}^{m}$.

$\langle 22\rangle \mathcal{M} \cap \mathcal{N}^{\perp}=\mathcal{M}^{\perp} \cap \mathcal{N}=\{0\}$ with some/all of the four facts: $\mathcal{M} \circ \mathcal{N}=\mathcal{N} \circ \mathcal{M}, \mathcal{M} \circ \mathcal{N}^{\perp}=\mathcal{N}^{\perp} \circ \mathcal{M}, \mathcal{M} \mathcal{L}^{\perp} \circ \mathcal{N}=\mathcal{N} \circ \mathcal{M}^{\perp}$, $\mathcal{M}^{\perp} \circ \mathcal{N}^{\perp}=\mathcal{N}^{\perp} \circ \mathcal{M}^{\perp}$.

$\langle 23\rangle \mathcal{M} \cap \mathcal{N}^{\perp}=\mathcal{M}^{\perp} \cap \mathcal{N}=\{0\}$ with one/both of the two facts: $\mathcal{M} \oplus\left(\mathcal{M}^{\perp} \cap \mathcal{N}^{\perp}\right)=\mathcal{N}^{\perp} \oplus(\mathcal{M} \cap \mathcal{N}), \mathcal{M}^{\perp} \oplus(\mathcal{M} \cap \mathcal{N})=$ $\mathcal{N} \oplus\left(\mathcal{M}^{\perp} \cap \mathcal{N}^{\perp}\right)$.

$\langle 24\rangle \mathcal{M} \cap \mathcal{N}^{\perp}=\mathcal{M}^{\perp} \cap \mathcal{N}=\{0\}$ with some/all of the four facts: $\mathcal{M} \bullet \mathcal{N}=\{0\}, \mathcal{N} \bullet \mathcal{M}=\{0\}, \mathcal{M}^{\perp} \bullet \mathcal{N}=\{0\}$, $\mathcal{N}^{\perp} \cdot \mathcal{M}=\{0\}$.

$\langle 25\rangle \mathcal{M} \cap \mathcal{N}^{\perp}=\mathcal{M}^{\perp} \cap \mathcal{N}=\{0\}$ with some/all of the four facts: $P_{\mathcal{M}}=P_{\mathcal{M} \cap \mathcal{N}}, P_{\mathcal{N}}=P_{\mathcal{M} \cap \mathcal{N}}, P_{\mathcal{M}^{\perp}}=P_{\mathcal{M}^{\perp} \cap \mathcal{N}^{\perp}}$, $P_{\mathcal{N}^{\perp}}=P_{\mathcal{M}^{\perp} \cap \mathcal{N}^{\perp}}$.

$\langle 26\rangle \mathcal{M} \cap \mathcal{N}^{\perp}=\mathcal{M}^{\perp} \cap \mathcal{N}=\{0\}$ with one/both of the two facts: $P_{\mathcal{M}}+P_{\mathcal{N}}=2 P_{\mathcal{M} \cap \mathcal{N}}, P_{\mathcal{M}^{\perp}}+P_{\mathcal{N}^{\perp}}=2 P_{\mathcal{M}^{\perp} \cap \mathcal{N}^{\perp}}$.

$\langle 27\rangle \mathcal{M} \cap \mathcal{N}^{\perp}=\mathcal{M}^{\perp} \cap \mathcal{N}=\{0\}$ with one/both of the two facts: $P_{\mathcal{M}+\mathcal{N}}=P_{\mathcal{M} \cap \mathcal{N}}, P_{\mathcal{M}^{\perp}+\mathcal{N}^{\perp}}=P_{\mathcal{M}^{\perp} \cap \mathcal{N}^{\perp}}$.

$\langle 28\rangle \mathcal{M} \cap \mathcal{N}^{\perp}=\mathcal{M}^{\perp} \cap \mathcal{N}=\{0\}$ and $I_{m}=P_{\mathcal{M} \cap \mathcal{N}}+P_{\mathcal{M}^{\perp} \cap \mathcal{N}^{\perp}}$.

$\langle 29\rangle \mathcal{M} \cap \mathcal{N}^{\perp}=\mathcal{M}^{\perp} \cap \mathcal{N}=\{0\}$ with some/all of the four facts: $\operatorname{dim}(\mathcal{M})=\operatorname{dim}(\mathcal{M} \cap \mathcal{N}), \operatorname{dim}(\mathcal{N})=\operatorname{dim}(\mathcal{M} \cap \mathcal{N})$, $\operatorname{dim}\left(\mathcal{M}^{\perp}\right)=\operatorname{dim}\left(\mathcal{M}^{\perp} \cap \mathcal{N}^{\perp}\right), \operatorname{dim}\left(\mathcal{N}^{\perp}\right)=\operatorname{dim}\left(\mathcal{M}^{\perp} \cap \mathcal{N}^{\perp}\right)$.

$\langle 30\rangle \mathcal{M} \cap \mathcal{N}^{\perp}=\mathcal{M}^{\perp} \cap \mathcal{N}=\{0\}$ with some/all of the four facts: $\operatorname{dim}(\mathcal{N}+\mathcal{N})=\operatorname{dim}(\mathcal{N}), \operatorname{dim}(\mathcal{M}+\mathcal{N})=\operatorname{dim}(\mathcal{N})$, $\operatorname{dim}\left(\mathcal{M}^{\perp}+\mathcal{N}^{\perp}\right)=\operatorname{dim}\left(\mathcal{M}^{\perp}\right), \operatorname{dim}\left(\mathcal{M}^{\perp}+\mathcal{N}^{\perp}\right)=\operatorname{dim}\left(\mathcal{N}^{\perp}\right)$. 
〈31 $\mathcal{M} \cap \mathcal{N}^{\perp}=\mathcal{M}^{\perp} \cap \mathcal{N}=\{0\}$ with some/all of the four facts: $\operatorname{dim}(\mathcal{M})+\operatorname{dim}\left(\mathcal{M}^{\perp} \cap \mathcal{N}^{\perp}\right)=m, \operatorname{dim}(\mathcal{N})+$ $\operatorname{dim}\left(\mathcal{M}^{\perp} \cap \mathcal{N}^{\perp}\right)=m, \operatorname{dim}\left(\mathcal{M}^{\perp}\right)+\operatorname{dim}(\mathcal{M} \cap \mathcal{N})=m, \operatorname{dim}\left(\mathcal{N}^{\perp}\right)+\operatorname{dim}(\mathcal{M} \cap \mathcal{N})=m$.

More equivalent statements for two subspaces to be equal will be given in Theorem 6.2.

Corollary 2.17. Let $\mathcal{N}$ and $\mathcal{N}$ be two linear subspaces of $\mathbb{C}^{m}$. Then, the following statements are equivalent:

$\langle 1\rangle \mathcal{M} \perp \mathcal{N}$, namely, $\mathcal{M}$ and $\mathcal{N}$ are orthogonal.

$\langle 2\rangle \mathcal{M} \subseteq \mathcal{N}^{\perp}$ and/or $\mathcal{M}^{\perp} \supseteq \mathcal{N}$.

33 $\mathcal{M}^{\perp}+\mathcal{N}=\mathcal{M}^{\perp}$ and/or $\mathcal{M}+\mathcal{N}^{\perp}=\mathcal{N}^{\perp}$.

(4) $\mathcal{M} \cap \mathcal{N}^{\perp}=\mathcal{M}$ and/or $\mathcal{M}^{\perp} \cap \mathcal{N}=\mathcal{N}$.

〈5) $\mathcal{M}=(\mathcal{M}+\mathcal{N}) \cap \mathcal{N}^{\perp}$ and/or $\mathcal{N}=\mathcal{M}^{\perp} \cap(\mathcal{M}+\mathcal{N})$.

<6) $\mathcal{M}^{\perp}=\left(\mathcal{M}^{\perp} \cap \mathcal{N}^{\perp}\right) \oplus \mathcal{N}$ and/or $\mathcal{N}^{\perp}=\mathcal{M} \oplus\left(\mathcal{M}^{\perp} \cap \mathcal{N}^{\perp}\right)$.

$\langle 7\rangle\left(\mathcal{M}^{\perp} \cap \mathcal{N}\right) \oplus\left(\mathcal{M}^{\perp} \cap \mathcal{N}^{\perp}\right) \oplus\left(\mathcal{M} \cap \mathcal{N}^{\perp}\right)=\mathbb{C}^{m}$ and $/$ or $\left(\mathcal{M}^{\perp}+\mathcal{N}\right) \cap(\mathcal{M}+\mathcal{N}) \cap\left(\mathcal{M}+\mathcal{N}^{\perp}\right)=\{0\}$.

$\langle 8\rangle \operatorname{dim}\left(\mathcal{M}^{\perp}+\mathcal{N}\right)=\operatorname{dim}\left(\mathcal{M}^{\perp}\right)$ and $/$ or $\operatorname{dim}\left(\mathcal{N}+\mathcal{N}^{\perp}\right)=\operatorname{dim}\left(\mathcal{N}^{\perp}\right)$.

$\langle 9\rangle \operatorname{dim}\left(\mathcal{M}^{\perp} \cap \mathcal{N}\right)=\operatorname{dim}(\mathcal{N})$ and/or $\operatorname{dim}\left(\mathcal{M} \cap \mathcal{N}^{\perp}\right)=\operatorname{dim}(\mathcal{M})$.

$\langle 10\rangle \operatorname{dim}\left(\mathcal{M}^{\perp} \cap \mathcal{N}\right)+\operatorname{dim}\left(\mathcal{M}^{\perp} \cap \mathcal{N}^{\perp}\right)+\operatorname{dim}\left(\mathcal{M} \cap \mathcal{N}^{\perp}\right)=m$.

$\langle 11\rangle \mathcal{M} \cap \mathcal{N}=\{0\}$ with some/all of the three facts: $\mathcal{N}^{\perp}$ and $\mathcal{N}$ are commutative, $\mathcal{M}^{\perp}$ and $\mathcal{N}^{\perp}$ are commutative, $\mathcal{M}$ and $\mathcal{N}^{\perp}$ are commutative.

$\langle 12\rangle \mathcal{M} \cap \mathcal{N}=\{0\}$ with one/both of the two facts: $\mathcal{N}=\left(\mathcal{M}^{\perp}+\mathcal{N}\right) \cap(\mathcal{M}+\mathcal{N}), \mathcal{M}=(\mathcal{M}+\mathcal{N}) \cap\left(\mathcal{M}+\mathcal{N}^{\perp}\right)$.

$\langle 13\rangle \mathcal{M} \cap \mathcal{N}=\{0\}$ with some/all of the eight facts: $\mathcal{M} \supseteq \mathcal{N} \circ \mathcal{M}, \mathcal{M} \supseteq \mathcal{N}^{\perp} \circ \mathcal{M}, \mathcal{M} \mathcal{N}^{\perp} \supseteq \mathcal{N} \circ \mathcal{M} \mathcal{L}^{\perp}, \mathcal{M}^{\perp} \supseteq \mathcal{N}^{\perp} \circ \mathcal{M}^{\perp}$, $\mathcal{N} \supseteq \mathcal{M}^{\perp} \circ \mathcal{N}, \mathcal{N} \supseteq \mathcal{M} \circ \mathcal{N}, \mathcal{N}^{\perp} \supseteq \mathcal{M}^{\perp} \circ \mathcal{N}^{\perp}, \mathcal{N}^{\perp} \supseteq \mathcal{M} \circ \mathcal{N}^{\perp}$.

$\left\langle\right.$ 14) $\mathcal{M} \cap \mathcal{N}=\{0\}$ with some/all of the six facts: $\mathcal{M} \subseteq\left(\mathcal{M} \cap \mathcal{N}^{\perp}\right) \oplus \mathcal{N}, \mathcal{N} \subseteq\left(\mathcal{M}^{\perp} \cap \mathcal{N}\right) \oplus \mathcal{M}, \mathcal{M}^{\perp} \subseteq\left(\mathcal{M}^{\perp} \cap \mathcal{N}\right) \oplus \mathcal{N}^{\perp}$, $\mathcal{M}^{\perp} \subseteq\left(\mathcal{M}^{\perp} \cap \mathcal{N}^{\perp}\right) \oplus \mathcal{N}, \mathcal{N}^{\perp} \subseteq\left(\mathcal{M}^{\perp} \cap \mathcal{N}^{\perp}\right) \oplus \mathcal{M}, \mathcal{N}^{\perp} \subseteq\left(\mathcal{M} \cap \mathcal{N}^{\perp}\right) \oplus \mathcal{M}^{\perp}$.

$\langle 15\rangle \mathcal{M} \cap \mathcal{N}=\{0\}$ with some/all of the three facts: $\mathcal{M}^{\perp} \circ \mathcal{N}=\mathcal{M}^{\perp} \cap \mathcal{N}, \mathcal{M}^{\perp} \circ \mathcal{N}^{\perp}=\mathcal{M}^{\perp} \cap \mathcal{N}^{\perp}, \mathcal{N}^{\perp} \circ \mathcal{M}^{\perp}=\mathcal{M}^{\perp} \cap \mathcal{N}^{\perp}$.

$\langle 16\rangle \mathcal{M} \circ \mathcal{N}=\{0\}$ and/or $\mathcal{N} \circ \mathcal{M}=\{0\}$.

$\langle 17\rangle \mathcal{M} \cap \mathcal{N}=\{0\}$ with one/both of the two facts: $\mathcal{M} \circ \mathcal{N}^{\perp}=\mathcal{M} \cap \mathcal{N}^{\perp}, \mathcal{N}^{\perp} \circ \mathcal{M}=\mathcal{M} \cap \mathcal{N}^{\perp}$.

$\langle 18\rangle \mathcal{M} \cap \mathcal{N}=\{0\}$ with one/both of the two facts: $\mathcal{M}^{\perp}+\mathcal{N}=\mathcal{N} \oplus\left(\mathcal{M}^{\perp} \cap \mathcal{N}^{\perp}\right), \mathcal{M}+\mathcal{N}^{\perp}=\mathcal{M} \oplus\left(\mathcal{M}^{\perp} \cap \mathcal{N}^{\perp}\right)$.

$\langle 19\rangle \mathcal{M}^{\perp} \oplus\left(\mathcal{M} \cap \mathcal{N}^{\perp}\right)=\mathbb{C}^{m}$ and/or $\mathcal{N}^{\perp} \oplus\left(\mathcal{M}^{\perp} \cap \mathcal{N}\right)=\mathbb{C}^{m}$.

$\langle 20\rangle \mathcal{M} \cap \mathcal{N}=\{0\}$ with one/both of the two facts: $\mathcal{M} \oplus \mathcal{N}=\mathcal{M} \oplus\left(\mathcal{M}^{\perp} \cap \mathcal{N}\right), \mathcal{M} \oplus \mathcal{N}=\mathcal{N} \oplus\left(\mathcal{M} \cap \mathcal{N}^{\perp}\right)$.

$\langle 21\rangle \mathcal{M} \cap \mathcal{N}=\{0\}$ with one/both of the two facts: $\mathcal{M}+\mathcal{N}^{\perp}=\mathcal{M} \oplus\left(\mathcal{M}^{\perp} \cap \mathcal{N}^{\perp}\right), \mathcal{N}+\mathcal{M}^{\perp}=\mathcal{N} \oplus\left(\mathcal{M}^{\perp} \cap \mathcal{N}^{\perp}\right)$.

$\langle 22\rangle \mathcal{M} \cap \mathcal{N}=\{0\}$ with one/both of the two facts: $\mathcal{M}^{\perp} \circ \mathcal{N}=\mathcal{N}, \mathcal{M} \circ \mathcal{N}^{\perp}=\mathcal{M}$.

$\langle 23\rangle \mathcal{M} \cap \mathcal{N}=\{0\}$ with one/both of the two facts: $\mathcal{N} \circ \mathcal{N}=\mathcal{N} \circ \mathcal{M}, \mathcal{M}^{\perp} \circ \mathcal{N}^{\perp}=\mathcal{N}^{\perp} \circ \mathcal{M}^{\perp}$.

<24) $\mathcal{M} \cap \mathcal{N}=\{0\}$ with one/both of the two facts: $\mathcal{M}^{\perp} \oplus\left(\mathcal{M} \cap \mathcal{N}^{\perp}\right)=\mathcal{N}^{\perp} \oplus\left(\mathcal{M}^{\perp} \cap \mathcal{N}\right), \mathcal{M} \oplus\left(\mathcal{M} \mathcal{M}^{\perp} \cap \mathcal{N}\right)=\mathcal{N} \oplus\left(\mathcal{N} \cap \mathcal{N}^{\perp}\right)$.

$\langle 25\rangle \mathcal{M} \cap \mathcal{N}=\{0\}$ with some/all of the three facts: $\mathcal{M}+\mathcal{N}=\left(\mathcal{M}^{\perp} \cap \mathcal{N}\right) \oplus\left(\mathcal{M} \cap \mathcal{N}^{\perp}\right), \mathcal{M}^{\perp}+\mathcal{N}=\left(\mathcal{M}^{\perp} \cap \mathcal{N}\right) \oplus$ $\left(\mathcal{M}^{\perp} \cap \mathcal{N}^{\perp}\right), \mathcal{M}+\mathcal{N}^{\perp}=\left(\mathcal{M}^{\perp} \cap \mathcal{N}^{\perp}\right) \oplus\left(\mathcal{M} \cap \mathcal{N}^{\perp}\right)$.

26) $\mathcal{M} \cap \mathcal{N}=\{0\}$ with some/all of the three facts: $\mathcal{M} \cap \mathcal{N}^{\perp}=(\mathcal{M}+\mathcal{N}) \cap\left(\mathcal{M}+\mathcal{N}^{\perp}\right), \mathcal{M}^{\perp} \cap \mathcal{N}=\left(\mathcal{M} \mathcal{L}^{\perp}+\mathcal{N}\right) \cap(\mathcal{N}+\mathcal{N})$, $\mathcal{M}^{\perp} \cap \mathcal{N}^{\perp}=\left(\mathcal{M}^{\perp}+\mathcal{N}\right) \cap\left(\mathcal{M}+\mathcal{N}^{\perp}\right)$.

$\langle 27\rangle \mathcal{M} \cap \mathcal{N}=\{0\}$ with one/both of the two facts: $\mathcal{M}^{\perp} \bullet \mathcal{N}=\{0\}, \mathcal{N}^{\perp} \bullet \mathcal{M}^{\perp}=\{0\}$

$\langle 28\rangle \mathcal{M} \cap \mathcal{N}=\{0\}$ with one/both of the two facts: $\mathcal{M}+\left(\mathcal{M}^{\perp} \cap \mathcal{N}\right)+\left(\mathcal{M}^{\perp} \cap \mathcal{N}^{\perp}\right)=\mathbb{C}^{m}, \mathcal{N}+\left(\mathcal{M} \mathcal{L}^{\perp} \cap \mathcal{N}^{\perp}\right)+\left(\mathcal{M} \cap \mathcal{N}^{\perp}\right)=$ $\mathbb{C}^{m}$.

$\langle 29\rangle \mathcal{M} \cap \mathcal{N}=\{0\}$ with one/both of the two facts: $P_{\mathcal{M}^{\perp}}=P_{\mathcal{M}^{\perp} \cap \mathcal{N}}+P_{\mathcal{M}^{\perp} \cap \mathcal{N}^{\perp}}, P_{\mathcal{N}}=P_{\mathcal{M}^{\perp} \cap \mathcal{N}}$.

$\langle 30\rangle \mathcal{M} \cap \mathcal{N}=\{0\}$ with one/both of the two facts: $P_{\mathcal{M}}=P_{\mathcal{M} \cap \mathcal{N}^{\perp}}, P_{\mathcal{N}^{\perp}}=P_{\mathcal{M}^{\perp} \cap \mathcal{N}^{\perp}}+P_{\mathcal{M} \cap \mathcal{N}^{\perp}}$.

$\langle 31\rangle \mathcal{M} \cap \mathcal{N}=\{0\}$ with one/both of the two facts: $P_{\mathcal{M}}+P_{\mathcal{N}^{\perp}}=P_{\mathcal{M}^{\perp} \cap \mathcal{N}^{\perp}}+2 P_{\mathcal{M}^{\prime} \cap \mathcal{N}^{\perp}}, P_{\mathcal{M}^{\perp}}+P_{\mathcal{N}}=2 P_{\mathcal{M}^{\perp} \cap \mathcal{N}}+$ $P_{\mathcal{M}^{\perp} \cap \mathcal{N}^{\perp}}$.

$\langle 32\rangle \mathcal{M} \cap \mathcal{N}=\{0\}$ with one/both of the two facts: $P_{\mathcal{M}^{+} \mathcal{N}^{\perp}}=P_{\mathcal{M}^{\perp} \cap \mathcal{N}^{\perp}}+P_{\mathcal{M} \cap \mathcal{N}^{\perp}}, P_{\mathcal{M}^{\perp}+\mathcal{N}}=P_{\mathcal{M}^{\perp} \cap \mathcal{N}^{+}}+P_{\mathcal{M}^{\perp} \cap \mathcal{N}^{\perp}}$.

$\langle 33\rangle \mathcal{M} \cap \mathcal{N}=\{0\}$ and $I_{m}=P_{\mathcal{M} \perp \perp \mathcal{N}}+P_{\mathcal{M}^{\perp} \cap \mathcal{N} \perp}+P_{\mathcal{M} \cap \mathcal{N}^{\perp}}$.

〈34〉 $\mathcal{M} \cap \mathcal{N}=\{0\}$ with one/both of the two facts: $\operatorname{dim}\left(\mathcal{M}^{\perp}\right)=\operatorname{dim}\left(\mathcal{M}^{\perp} \cap \mathcal{N}\right)+\operatorname{dim}\left(\mathcal{M}^{\perp} \cap \mathcal{N}^{\perp}\right), \operatorname{dim}\left(\mathcal{N}^{\perp}\right)=$ $\operatorname{dim}\left(\mathcal{M}^{\perp} \cap \mathcal{N}^{\perp}\right)+\operatorname{dim}\left(\mathcal{M} \cap \mathcal{N}^{\perp}\right)$.

$\langle 35\rangle \mathcal{M} \cap \mathcal{N}=\{0\}$ and $\operatorname{dim}\left(\mathcal{M}^{\perp}+\mathcal{N}\right)=\operatorname{dim}\left(\mathcal{M}^{\perp} \cap \mathcal{N}\right)+\operatorname{dim}\left(\mathcal{M}^{\perp} \cap \mathcal{N}^{\perp}\right)$.

36) $\mathcal{M} \cap \mathcal{N}=\{0\}$ and $\operatorname{dim}(\mathcal{M}+\mathcal{N})=\operatorname{dim}\left(\mathcal{M}^{\perp} \cap \mathcal{N}\right)+\operatorname{dim}\left(\mathcal{M} \cap \mathcal{N}^{\perp}\right)$.

337) $\mathcal{M} \cap \mathcal{N}=\{0\}$ and $\operatorname{dim}\left(\mathcal{N}+\mathcal{N}^{\perp}\right)=\operatorname{dim}\left(\mathcal{M}^{\perp} \cap \mathcal{N}^{\perp}\right)+\operatorname{dim}\left(\mathcal{M} \cap \mathcal{N}^{\perp}\right)$. 
〈38> $\mathcal{M} \cap \mathcal{N}=\{0\}$ and $\operatorname{dim}\left(\mathcal{M}^{\perp}+\mathcal{N}^{\perp}\right)=\operatorname{dim}\left(\mathcal{M}^{\perp} \cap \mathcal{N}\right)+\operatorname{dim}\left(\mathcal{M}^{\perp} \cap \mathcal{N}^{\perp}\right)+\operatorname{dim}\left(\mathcal{M} \cap \mathcal{N}^{\perp}\right)$.

〈39) $\mathcal{M} \cap \mathcal{N}=\{0\}$ and with some/all of the five facts: $\operatorname{dim}\left(\mathcal{M}^{\perp} \circ \mathcal{N}\right)=\operatorname{dim}\left(\mathcal{M}^{\perp} \cap \mathcal{N}\right), \operatorname{dim}\left(\mathcal{M}^{\perp} \circ \mathcal{N}^{\perp}\right)=\operatorname{dim}\left(\mathcal{M}^{\perp} \cap\right.$ $\left.\mathcal{N}^{\perp}\right), \operatorname{dim}\left(\mathcal{N}^{\perp} \circ \mathcal{M}^{\perp}\right)=\operatorname{dim}\left(\mathcal{M}^{\perp} \cap \mathcal{N}^{\perp}\right), \operatorname{dim}\left(\mathcal{M} \circ \mathcal{N}^{\perp}\right)=\operatorname{dim}\left(\mathcal{M} \cap \mathcal{N}^{\perp}\right), \operatorname{dim}\left(\mathcal{N}^{\perp} \circ \mathcal{M}\right)=\operatorname{dim}\left(\mathcal{M} \cap \mathcal{N}^{\perp}\right)$.

$\langle 40\rangle \mathcal{M} \cap \mathcal{N}=\{0\}$ with some/all of the four facts: $\operatorname{dim}\left(\mathcal{M}^{\perp}+\mathcal{N}\right)=\operatorname{dim}(\mathcal{N})+\operatorname{dim}\left(\mathcal{M}^{\perp} \cap \mathcal{N}^{\perp}\right), \operatorname{dim}(\mathcal{N}+\mathcal{N})=$ $\operatorname{dim}(\mathcal{M})+\operatorname{dim}\left(\mathcal{M}^{\perp} \cap \mathcal{N}\right), \operatorname{dim}(\mathcal{N}+\mathcal{N})=\operatorname{dim}(\mathcal{N})+\operatorname{dim}\left(\mathcal{M} \cap \mathcal{N}^{\perp}\right), \operatorname{dim}\left(\mathcal{M}+\mathcal{N}^{\perp}\right)=\operatorname{dim}(\mathcal{N})+\operatorname{dim}\left(\mathcal{M}^{\perp} \cap \mathcal{N}^{\perp}\right)$.

Descriptions of a sequence of linear subspaces are very complicated problems in linear algebra. Some previous considerations on three or more linear subspaces and their joint positions were scattered in the literature associated with pure algebra and Hilbert space; see e.g., [47, 66, 68, 70, 94, 95, 108, 139].

\section{The simultaneous CS decomposition of two orthogonal projectors and their consequences}

Let $\mathcal{M}$ and $\mathcal{N}$ be two linear subspaces of $\mathbb{C}^{m}$. An essential characterization of the relative position of the two subspaces is to define canonical angles between the vectors in them, which measure how the two subspaces differ in a natural way. Many results related to canonical angles between two subspaces can be found in the literature; see e.g., [31, 37, 42, 53, 55, 56, 71, 72, 106, 107, 124, 150, 151]. In particular, canonical angles between two subspaces can be used to construct simultaneous decomposition identities of the two projectors onto the two subspaces. During this approach, various matrix decompositions, including the well-known CS decomposition, can be used; see, e.g., [27, 28, 42, 71, 74, 79, 86, 114, 130, 150]. In this section, we reconsider the CS decomposition for two orthogonal projectors, and use the decomposition with precise sizes of submatrices to characterize many behaviors of the pair of orthogonal projectors and their operations.

Lemma 3.1. Let $\mathcal{M}$ and $\mathcal{N}$ be two linear subspaces of $\mathbb{C}^{m}$, and define

$$
\begin{array}{lll}
A=P_{\mathcal{M}}, & B=P_{\mathcal{N}}, \\
\widetilde{A}=P_{\mathcal{M}^{\perp}}=I_{m}-P_{\mathcal{M}}=I_{m}-A, & \widetilde{B}=P_{\mathcal{N}^{\perp}}=I_{m}-P_{\mathcal{N}}=I_{m}-B .
\end{array}
$$

Then, there exists a unitary matrix $U$ such that

$$
\begin{aligned}
& A=U \operatorname{diag}\left(I_{k_{1}}, 0_{k_{2}}, I_{k_{3}}, I_{k_{4}}, 0_{k_{5}}, 0_{k_{6}}\right) U^{*}, \\
& B=U \operatorname{diag}\left(\left[\begin{array}{cc}
C^{2} & C S \\
C S & S^{2}
\end{array}\right], I_{k_{3}}, 0_{k_{4}}, I_{k_{5}}, 0_{k_{6}}\right) U^{*}, \\
& \widetilde{A}=U \operatorname{diag}\left(0_{k_{1}}, I_{k_{2}}, 0_{k_{3}+k_{4}}, I_{k_{5}+k_{6}}\right) U^{*}, \\
& \widetilde{B}=U \operatorname{diag}\left(\left[\begin{array}{cc}
S^{2} & -C S \\
-C S & C^{2}
\end{array}\right], 0_{k_{3}}, I_{k_{4}}, 0_{k_{5}}, I_{k_{6}}\right) U^{*},
\end{aligned}
$$

where $C$ and $S$ are positive diagonal real matrices such that

$$
C^{2}+S^{2}=I_{k_{1}}
$$

and the sizes of the submatrices in (3.3)-(3.6) are given by

$$
\begin{aligned}
& k_{1}=k_{2}=r[A, B]-r(A)-r(B)+r(A B), \quad k_{3}=r(A)+r(B)-r[A, B], \\
& k_{4}=r(A)-r(A B), \quad k_{5}=r(B)-r(A B), \quad k_{6}=m-r[A, B], \\
& 0 \leqslant k_{1} \leqslant m / 2, \quad 0 \leqslant k_{3}, k_{4}, k_{5}, k_{6} \leqslant m .
\end{aligned}
$$

A special case of Lemma 3.1 is given below. 
Corollary 3.2. Let $M \in \mathbb{C}^{m \times m}$. Then, there exists a unitary matrix $U$ such that

$$
\begin{aligned}
M M^{\dagger} & =U \operatorname{diag}\left(I_{k_{1}}, 0_{k_{2}}, I_{k_{3}+k_{4}}, 0_{k_{5}+k_{6}}\right) U^{*}, \\
M^{\dagger} M & =U \operatorname{diag}\left(\left[\begin{array}{cc}
C^{2} & C S \\
C S & S^{2}
\end{array}\right], I_{k_{3}}, 0_{k_{4}}, I_{k_{5}}, 0_{k_{6}}\right) U^{*}, \\
I_{m}-M M^{\dagger} & =U \operatorname{diag}\left(0_{k_{1}}, I_{k_{2}}, 0_{k_{3}+k_{4}}, I_{k_{5}+k_{6}}\right) U^{*}, \\
I_{m}-M^{\dagger} M & =U \operatorname{diag}\left(\left[\begin{array}{cc}
S^{2} & -C S \\
-C S & C^{2}
\end{array}\right], 0_{k_{3}}, I_{k_{4}}, 0_{k_{5}}, I_{k_{6}}\right) U^{*},
\end{aligned}
$$

where $C$ and $S$ are positive diagonal real matrices such that $C^{2}+S^{2}=I_{k_{1}}$, the sizes of the blocks in (3.11)-(3.14) are given by

$$
\begin{aligned}
& k_{1}=k_{2}=r\left[M, M^{*}\right]-2 r(M)+r\left(M^{2}\right), \quad k_{3}=2 r(M)-r\left[M, M^{*}\right], \\
& k_{4}=k_{5}=r(M)-r\left(M^{2}\right), \quad k_{6}=m-r\left[M, M^{*}\right] .
\end{aligned}
$$

In particular, under the condition $r\left(M^{2}\right)=r(M)$, (3.11)-(3.16) reduce to

$$
\begin{aligned}
& M M^{\dagger}=U \operatorname{diag}\left(I_{k_{1}}, 0_{k_{2}}, I_{k_{3}}, 0_{k_{6}}\right) U^{*}, \quad M^{\dagger} M=U \operatorname{diag}\left(\left[\begin{array}{cc}
C^{2} & C S \\
C S & S^{2}
\end{array}\right], I_{k_{3}}, 0_{k_{6}}\right) U^{*}, \\
& I_{m}-M M^{\dagger}=U \operatorname{diag}\left(0_{k_{1}}, I_{k_{2}}, 0_{k_{3}}, I_{k_{6}}\right) U^{*}, I_{m}-M^{\dagger} M=U \operatorname{diag}\left(\left[\begin{array}{cc}
S^{2} & -C S \\
-C S & C^{2}
\end{array}\right], 0_{k_{3}}, I_{k_{6}}\right) U^{*},
\end{aligned}
$$

where $k_{1}=k_{2}=r\left[M, M^{*}\right]-r(M), k_{3}=2 r(M)-r\left[M, M^{*}\right]$ and $k_{6}=m-r\left[M, M^{*}\right]$. Under the condition $M^{2}=0$, (3.11)-(3.16) reduce to

$$
\begin{aligned}
& M M^{\dagger}=U \operatorname{diag}\left(I_{k_{4}}, 0_{k_{5}+k_{6}}\right) U^{*}, \quad M^{\dagger} M=U \operatorname{diag}\left(0_{k_{4}}, I_{k_{5}}, 0_{k_{6}}\right) U^{*}, \\
& I_{m}-M M^{\dagger}=U \operatorname{diag}\left(0_{k_{4}}, I_{k_{5}+k_{6}}\right) U^{*}, I_{m}-M^{\dagger} M=U \operatorname{diag}\left(I_{k_{4}}, 0_{k_{5}}, I_{k_{6}}\right) U^{*}
\end{aligned}
$$

where $k_{4}=k_{5}=r(M)$ and $k_{6}=m-2 r(M)$.

Lemma 3.3. Any Hermitian matrix $M$ can uniquely be decomposed as $M=M_{+}+M_{-}$, where $M_{+}$and $M_{-}$, called the positive semi-definite and negative semi-definite parts of $M$, satisfy

$$
M_{+} \succcurlyeq 0, \quad M_{-} \preccurlyeq 0, \quad M_{+} M_{-}=M_{-} M_{+}=0 .
$$

In this situation, the following results hold.
(a) $M_{+}=\frac{1}{2}\left[\left(M^{2}\right)^{1 / 2}+M\right], M_{-}=\frac{1}{2}\left[M-\left(M^{2}\right)^{1 / 2}\right]$, and $M_{+}-M_{-}=\left(M^{2}\right)^{1 / 2}$.
(b) $\left(M^{k}\right)_{+}=\left(M_{+}\right)^{k},\left(M^{2 k}\right)_{-}=0$, and $\left(M^{2 k-1}\right)_{-}=\left(M_{-}\right)^{2 k-1}$.
(c) $M^{k}=\left(M_{+}\right)^{k}+\left(M_{-}\right)^{k}$.
(d) $\left(M_{+}\right)^{\dagger}=\left(M^{\dagger}\right)_{+}$and $\left(M_{-}\right)^{\dagger}=\left(M^{\dagger}\right)_{-}$.
(e) $M^{\dagger}=M_{+}^{\dagger}+M_{-}^{\dagger}$.
(f) $i_{+}(M)=r\left(M_{+}\right), i_{-}(M)=r\left(M_{-}\right)$, and $r\left(M_{+}\right)+r\left(M_{-}\right)=r(M)$.
(g) $\mathscr{R}\left(M_{+}\right) \subseteq \mathscr{R}(M), \mathscr{R}\left(M_{-}\right) \subseteq \mathscr{R}(M)$, and $\mathscr{R}\left(M_{+}\right) \oplus \mathscr{R}\left(M_{-}\right)=\mathscr{R}(M)$.

Proof. It follows directly from the spectral decomposition of Hermitian matrix.

A most valuable part of Lemma 3.1 is giving the analytical formulas for calculating the sizes of all submatrices in (3.3), which enable us to precisely characterize many algebraic properties of the two orthogonal projectors and their operations. In what follows, we give a variety of simultaneous decomposition identities of $A \pm B$, $A B, B A, A B \pm B A, A B A, B A B, A B A \pm B A B$, as well as $\mathscr{R}(A) \cap \mathscr{R}(B), \mathscr{R}(A) \cap \mathscr{R}(\widetilde{B}), \mathscr{R}(\widetilde{A}) \cap \mathscr{R}(B)$ and $\mathscr{R}(\widetilde{A}) \cap \mathscr{R}(\widetilde{B})$, which allow us to characterize behaviors of orthogonal projectors and their operations in many cases. It can be seen in particular that the diagonal elements of $C^{2}$ are the singular values of the product of $A B$ (eigenvalues of the product of $A B A$ ) less than 1 . 
Theorem 3.4. Let $\mathcal{M}$ and $\mathcal{N}$ be two linear subspaces of $\mathbb{C}^{m}$, and assume that $A, B, \widetilde{A}$ and $\widetilde{B}$ in (3.2) are decomposed as (3.3)-(3.6), respectively. Also denote $\widehat{C}=I_{k_{1}}+C$ and $\widehat{S}=I_{k_{1}}+S$. Then, the following results hold.

(a) There exists a unitary matrix $U$ such that the following results hold simultaneously

$$
\begin{aligned}
& A+B=U \operatorname{diag}\left(\left[\begin{array}{cc}
I_{k_{1}}+C^{2} & C S \\
C S & S^{2}
\end{array}\right], 2 I_{k_{3}}, I_{k_{4}+k_{5}}, 0_{k_{6}}\right) U^{*}, \\
& (A+B)^{1 / 2}=U \operatorname{diag}\left(\frac{1}{\sqrt{2}}\left[\begin{array}{cc}
\widehat{S}^{1 / 2}+C^{2} \widehat{S}^{-1 / 2} & C S \widehat{S}^{-1 / 2} \\
C S \widehat{S}^{-1 / 2} & S \widehat{S}^{1 / 2}
\end{array}\right], \sqrt{2} I_{k_{3}}, I_{k_{4}+k_{5}}, 0_{k_{6}}\right) U^{*}, \\
& I_{m}+A-B=U \operatorname{diag}\left(\left[\begin{array}{cc}
I_{k_{1}}+S^{2} & -C S \\
-C S & C^{2}
\end{array}\right], I_{k_{3}}, 2 I_{k_{4}}, 0_{k_{5}}, I_{k_{6}}\right) U^{*} \text {, } \\
& \left(I_{m}+A-B\right)^{1 / 2}=U \operatorname{diag}\left(\frac{1}{\sqrt{2}}\left[\begin{array}{cc}
\widehat{C}^{1 / 2}+S^{2} \widehat{C}^{-1 / 2} & -C S \widehat{C}^{-1 / 2} \\
-C S \widehat{C}^{-1 / 2} & C \widehat{C}^{1 / 2}
\end{array}\right], I_{k_{3}}, \sqrt{2} I_{k_{4}}, 0_{k_{5}}, I_{k_{6}}\right) U^{*}, \\
& I_{m}-A+B=U \operatorname{diag}\left(\left[\begin{array}{cc}
C^{2} & C S \\
C S & I_{k_{1}}+S^{2}
\end{array}\right], I_{k_{3}}, 0_{k_{4}}, 2 I_{k_{5}}, I_{k_{6}}\right) U^{*} \text {, } \\
& \left(I_{m}-A+B\right)^{1 / 2}=U \operatorname{diag}\left(\frac{1}{\sqrt{2}}\left[\begin{array}{cc}
C \widehat{C}^{1 / 2} & C S \widehat{C}^{-1 / 2} \\
\operatorname{CS} \widehat{C}^{-1 / 2} & \widehat{C}^{1 / 2}+S^{2} \widehat{C}^{-1 / 2}
\end{array}\right], I_{k_{3}}, 0_{k_{4}}, \sqrt{2} I_{k_{5}}, I_{k_{6}}\right) U^{*}, \\
& I_{m}-A-B=U \operatorname{diag}\left(\left[\begin{array}{cc}
-C^{2} & -C S \\
-C S & C^{2}
\end{array}\right],-I_{k_{3}}, 0_{k_{4}+k_{5}}, I_{k_{6}}\right) U^{*}, \\
& \left(I_{m}-A-B\right)^{2}=U \operatorname{diag}\left(C^{2}, C^{2}, I_{k_{3}}, 0_{k_{4}+k_{5}}, I_{k_{6}}\right) U^{*} \text {, } \\
& {\left[\left(I_{m}-A-B\right)^{2}\right]^{1 / 2}=U \operatorname{diag}\left(C, C, I_{k_{3}}, 0_{k_{4}+k_{5}}, I_{k_{6}}\right) U^{*} \text {, }} \\
& A-B=U \operatorname{diag}\left(\left[\begin{array}{cc}
S^{2} & -C S \\
-C S & -S^{2}
\end{array}\right], 0_{k_{3}}, I_{k_{4}},-I_{k_{5}}, 0_{k_{6}}\right) U^{*} \text {, } \\
& (A-B)^{2}=U \operatorname{diag}\left(S^{2}, S^{2}, 0_{k_{3}}, I_{k_{4}+k_{5}}, 0_{k_{6}}\right) U^{*} \text {, } \\
& {\left[(A-B)^{2}\right]^{1 / 2}=U \operatorname{diag}\left(S, S, 0_{k_{3}}, I_{k_{4}+k_{5}}, O_{k_{6}}\right) U^{*} \text {, }} \\
& 2 I_{m}-A-B=U \operatorname{diag}\left(\left[\begin{array}{cc}
S^{2} & -C S \\
-C S & I_{k_{1}}+C^{2}
\end{array}\right], 0_{k_{3}}, I_{k_{4}+k_{5}}, 2 I_{k_{6}}\right) U^{*}, \\
& \left(2 I_{m}-A-B\right)^{1 / 2}=U \operatorname{diag}\left(\frac{1}{\sqrt{2}}\left[\begin{array}{cc}
S \widehat{S}^{1 / 2} & -C S \widehat{S}^{-1 / 2} \\
-C S \widehat{S}^{-1 / 2} & \widehat{S}^{1 / 2}+C^{2} \widehat{S}^{-1 / 2}
\end{array}\right], 0_{k_{3}}, I_{k_{4}+k_{5}}, \sqrt{2} I_{k_{6}}\right) U^{*}, \\
& A B=U \operatorname{diag}\left(\left[\begin{array}{cc}
C^{2} & C S \\
0 & 0
\end{array}\right], I_{k_{3}}, 0_{k_{4}+k_{5}+k_{6}}\right) U^{*},(A B)^{1 / 2}=U \operatorname{diag}\left(\left[\begin{array}{cc}
C & S \\
0 & 0
\end{array}\right], I_{k_{3}}, 0_{k_{4}+k_{5}+k_{6}}\right) U^{*} \text {, } \\
& B A=U \operatorname{diag}\left(\left[\begin{array}{cc}
C^{2} & 0 \\
C S & 0
\end{array}\right], I_{k_{3}}, 0_{k_{4}+k_{5}+k_{6}}\right) U^{*},(B A)^{1 / 2}=U \operatorname{diag}\left(\left[\begin{array}{cc}
C & 0 \\
S & 0
\end{array}\right], I_{k_{3}}, 0_{k_{4}+k_{5}+k_{6}}\right) U^{*} \text {, } \\
& A+B-A B=U \operatorname{diag}\left(\left[\begin{array}{cc}
I_{k_{1}} & 0 \\
C S & S^{2}
\end{array}\right], I_{k_{3}+k_{4}+k_{5}}, 0_{k_{6}}\right) U^{*}, \\
& (A+B-A B)^{1 / 2}=U \operatorname{diag}\left(\left[\begin{array}{cc}
I_{k_{1}} & 0 \\
C S \hat{S}^{-1} & S
\end{array}\right], I_{k_{3}+k_{4}+k_{5}}, 0_{k_{6}}\right) U^{*}, \\
& A+B-B A=U \operatorname{diag}\left(\left[\begin{array}{cc}
I_{k_{1}} & C S \\
0 & S^{2}
\end{array}\right], I_{k_{3}+k_{4}+k_{5}}, 0_{k_{6}}\right) U^{*}, \\
& (A+B-B A)^{1 / 2}=U \operatorname{diag}\left(\left[\begin{array}{cc}
I_{k_{1}} & C S \hat{S}^{-1} \\
0 & S
\end{array}\right], I_{k_{3}+k_{4}+k_{5}}, 0_{k_{6}}\right) U^{*} \text {, }
\end{aligned}
$$




$$
\begin{aligned}
& A B+B A=U \operatorname{diag}\left(\left[\begin{array}{cc}
2 C^{2} & C S \\
C S & 0
\end{array}\right], 2 I_{k_{3}}, 0_{k_{4}+k_{5}+k_{6}}\right) U^{*}, \\
& 2 I_{m}-A B-B A=U \operatorname{diag}\left(\left[\begin{array}{cc}
2 S^{2} & -C S \\
-C S & 2 I_{k_{1}}
\end{array}\right], 0_{k_{3}}, 2 I_{k_{4}+k_{5}+k_{6}}\right) U^{*}, \\
& 4^{-1} I_{m}+A B+B A=\left(2^{-1} I_{m}-A-B\right)^{2} \\
& =U \operatorname{diag}\left(\left[\begin{array}{cc}
4^{-1} I_{m}+2 C^{2} & C S \\
C S & 4^{-1} I_{m}
\end{array}\right],\left(2+4^{-1}\right) I_{k_{3}}, 4^{-1} I_{k_{4}+k_{5}+k_{6}}\right) U^{*} \text {, } \\
& A B-B A=U \operatorname{diag}\left(\left[\begin{array}{cc}
0 & C S \\
-C S & 0
\end{array}\right], 0_{m-2 k_{1}}\right) U^{*}, \\
& (A B-B A)^{2}=-U \operatorname{diag}\left(C^{2} S^{2}, C^{2} S^{2}, 0_{m-2 k_{1}}\right) U^{*}, \\
& {\left[(A B-B A)^{2}\right]^{1 / 2}=\sqrt{-1} U \operatorname{diag}\left(C S, C S, 0_{m-2 k_{1}}\right) U^{*},} \\
& I_{m}-A B=U \operatorname{diag}\left(\left[\begin{array}{cc}
S^{2} & -C S \\
0 & I_{k_{1}}
\end{array}\right], 0_{k_{3}}, I_{k_{4}+k_{5}+k_{6}}\right) U^{*}, \\
& \left(I_{m}-A B\right)^{1 / 2}=U \operatorname{diag}\left(\left[\begin{array}{cc}
S & -C S \hat{S}^{-1} \\
0 & I_{k_{1}}
\end{array}\right], 0_{k_{3}}, I_{k_{4}+k_{5}+k_{6}}\right) U^{*}, \\
& I_{m}-B A=U \operatorname{diag}\left(\left[\begin{array}{cc}
S^{2} & 0 \\
-C S & I_{k_{1}}
\end{array}\right], 0_{k_{3}}, I_{k_{4}+k_{5}+k_{6}}\right) U^{*} \text {, } \\
& \left(I_{m}-B A\right)^{1 / 2}=U \operatorname{diag}\left(\left[\begin{array}{cc}
S & 0 \\
-C S \hat{S}^{-1} & I_{k_{1}}
\end{array}\right], 0_{k_{3}}, I_{k_{4}+k_{5}+k_{6}}\right) U^{*}, \\
& A \widetilde{B}=U \operatorname{diag}\left(\left[\begin{array}{cc}
S^{2} & -C S \\
0 & 0
\end{array}\right], 0_{k_{3}}, I_{k_{4}}, 0_{k_{5}+k_{6}}\right) U^{*} \text {, } \\
& (A \widetilde{B})^{1 / 2}=U \operatorname{diag}\left(\left[\begin{array}{cc}
S & -C \\
0 & 0
\end{array}\right], 0_{k_{3}}, I_{k_{4}}, 0_{k_{5}+k_{6}}\right) U^{*}, \\
& \widetilde{B} A=U \operatorname{diag}\left(\left[\begin{array}{cc}
S^{2} & 0 \\
-C S & 0
\end{array}\right], 0_{k_{3}}, I_{k_{4}}, 0_{k_{5}+k_{6}}\right) U^{*} \text {, } \\
& (\widetilde{B} A)^{1 / 2}=U \operatorname{diag}\left(\left[\begin{array}{cc}
S & 0 \\
-C & 0
\end{array}\right], 0_{k_{3}}, I_{k_{4}}, 0_{k_{5}+k_{6}}\right) U^{*}, \\
& B \widetilde{A}=U \operatorname{diag}\left(\left[\begin{array}{cc}
0 & C S \\
0 & S^{2}
\end{array}\right], 0_{k_{3}+k_{4}}, I_{k_{5}}, 0_{k_{6}}\right) U^{*} \text {, } \\
& (B \widetilde{A})^{1 / 2}=U \operatorname{diag}\left(\left[\begin{array}{ll}
0 & C \\
0 & S
\end{array}\right], 0_{k_{3}+k_{4}}, I_{k_{5}}, 0_{k_{6}}\right) U^{*}, \\
& \tilde{A} B=U \operatorname{diag}\left(\left[\begin{array}{cc}
0 & 0 \\
C S & S^{2}
\end{array}\right], 0_{k_{3}+k_{4}}, I_{k_{5}}, 0_{k_{6}}\right) U^{*} \text {, } \\
& (\widetilde{A} B)^{1 / 2}=U \operatorname{diag}\left(\left[\begin{array}{ll}
0 & 0 \\
C & S
\end{array}\right], 0_{k_{3}+k_{4}}, I_{k_{5}}, 0_{k_{6}}\right) U^{*}, \\
& \widetilde{A} \widetilde{B}=U \operatorname{diag}\left(\left[\begin{array}{cc}
0 & 0 \\
-C S & C^{2}
\end{array}\right], 0_{k_{3}+k_{4}+k_{5}}, I_{k_{6}}\right) U^{*}, \\
& (\widetilde{A} \widetilde{B})^{1 / 2}=U \operatorname{diag}\left(\left[\begin{array}{cc}
0 & 0 \\
-S & C
\end{array}\right], 0_{k_{3}+k_{4}+k_{5}}, I_{k_{6}}\right) U^{*},
\end{aligned}
$$




$$
\begin{aligned}
& \widetilde{B} \widetilde{A}=U \operatorname{diag}\left(\left[\begin{array}{cc}
0 & -C S \\
0 & C^{2}
\end{array}\right], 0_{k_{3}+k_{4}+k_{5}}, I_{k_{6}}\right) U^{*}, \\
& (\widetilde{B} \widetilde{A})^{1 / 2}=U \operatorname{diag}\left(\left[\begin{array}{cc}
0 & -S \\
0 & C
\end{array}\right], 0_{k_{3}+k_{4}+k_{5}}, I_{k_{6}}\right) U^{*}, \\
& A \widetilde{B}+\widetilde{B} A=U \operatorname{diag}\left(\left[\begin{array}{cc}
2 S^{2} & -C S \\
-C S & 0
\end{array}\right], 0_{k_{3}}, 2 I_{k_{4}}, 0_{k_{5}+k_{6}}\right) U^{*} \text {, } \\
& \widetilde{A} B+B \widetilde{A}=U \operatorname{diag}\left(\left[\begin{array}{cc}
0 & C S \\
C S & 2 S^{2}
\end{array}\right], 0_{k_{3}+k_{4}}, 2 I_{k_{5}}, 0_{k_{6}}\right) U^{*} \text {, } \\
& \widetilde{A} \widetilde{B}+\widetilde{B} \widetilde{A}=U \operatorname{diag}\left(\left[\begin{array}{cc}
0 & -C S \\
-C S & 2 C^{2}
\end{array}\right], 0_{k_{3}+k_{4}+k_{5}}, 2 I_{k_{6}}\right) U^{*} \text {, } \\
& A B A=U \operatorname{diag}\left(C^{2}, 0_{k_{2}}, I_{k_{3}}, 0_{k_{4}+k_{5}+k_{6}}\right) U^{*}, \\
& (A B A)^{1 / 2}=U \operatorname{diag}\left(C, 0_{k_{2}}, I_{k_{3}}, 0_{k_{4}+k_{5}+k_{6}}\right) U^{*}, \\
& B A B=U \operatorname{diag}\left(\left[\begin{array}{cc}
C^{4} & C^{3} S \\
C^{3} S & C^{2} S^{2}
\end{array}\right], I_{k_{3}}, 0_{k_{4}+k_{5}+k_{6}}\right) U^{*}, \\
& (B A B)^{1 / 2}=U \operatorname{diag}\left(\left[\begin{array}{cc}
C^{3} & C^{2} S \\
C^{2} S & C S^{2}
\end{array}\right], I_{k_{3}}, 0_{k_{4}+k_{5}+k_{6}}\right) U^{*}, \\
& A B \widetilde{A}=-A \widetilde{B} \widetilde{A}=U \operatorname{diag}\left(\left[\begin{array}{cc}
0 & C S \\
0 & 0
\end{array}\right], 0_{m-2 k_{1}}\right) U^{*} \text {, } \\
& \widetilde{A} B A=-\widetilde{A} \widetilde{B} A=U \operatorname{diag}\left(\left[\begin{array}{cc}
0 & 0 \\
C S & 0
\end{array}\right], 0_{m-2 k_{1}}\right) U^{*}, \\
& B A \widetilde{B}=-B \widetilde{A} \widetilde{B}=U \operatorname{diag}\left(\left[\begin{array}{cc}
C^{2} S^{2} & -C^{3} S \\
C S^{3} & -C^{2} S^{2}
\end{array}\right], 0_{m-2 k_{1}}\right) U^{*} \text {, } \\
& \widetilde{B} A B=-\widetilde{B} \widetilde{A} B=U \operatorname{diag}\left(\left[\begin{array}{cc}
C^{2} S^{2} & C S^{3} \\
-C^{3} S & -C^{2} S^{2}
\end{array}\right], 0_{m-2 k_{1}}\right) U^{*} \text {, } \\
& A \widetilde{B} A=U \operatorname{diag}\left(S^{2}, 0_{k_{2}+k_{3}}, I_{k_{4}}, 0_{k_{5}+k_{6}}\right) U^{*} \text {, } \\
& (A \widetilde{B} A)^{1 / 2}=U \operatorname{diag}\left(S, 0_{k_{2}+k_{3}}, I_{k_{4}}, 0_{k_{5}+k_{6}}\right) U^{*}, \\
& B \widetilde{A} B=U \operatorname{diag}\left(\left[\begin{array}{cc}
C^{2} S^{2} & C S^{3} \\
C S^{3} & S^{4}
\end{array}\right], 0_{m-2 k_{1}}\right) U^{*}, \\
& (B \widetilde{A} B)^{1 / 2}=U \operatorname{diag}\left(\left[\begin{array}{cc}
C^{2} S & C S^{2} \\
C S^{2} & S^{3}
\end{array}\right], 0_{m-2 k_{1}}\right) U^{*}, \\
& \widetilde{A} B \widetilde{A}=U \operatorname{diag}\left(0_{k_{1}}, S^{2}, 0_{k_{3}+k_{4}}, I_{k_{5}}, 0_{k_{6}}\right) U^{*} \text {, } \\
& (\widetilde{A} B \widetilde{A})^{1 / 2}=U \operatorname{diag}\left(0_{k_{1}}, S, 0_{k_{3}+k_{4}}, I_{k_{5}}, 0_{k_{6}}\right) U^{*} \text {, } \\
& \widetilde{B} A \widetilde{B}=U \operatorname{diag}\left(\left[\begin{array}{cc}
S^{4} & -C S^{3} \\
-C S^{3} & C^{2} S^{2}
\end{array}\right], 0_{k_{3}}, I_{k_{4}}, 0_{k_{5}+k_{6}}\right) U^{*} \text {, } \\
& (\widetilde{B} A \widetilde{B})^{1 / 2}=U \operatorname{diag}\left(\left[\begin{array}{cc}
S^{3} & -C S^{2} \\
-C S^{2} & C^{2} S
\end{array}\right], 0_{k_{3}}, I_{k_{4}}, 0_{k_{5}+k_{6}}\right) U^{*} \text {, } \\
& \widetilde{A} \widetilde{B} \widetilde{A}=U \operatorname{diag}\left(0_{k_{1}}, C^{2}, 0_{k_{3}+k_{4}+k_{5}}, I_{k_{6}}\right) U^{*} \text {, } \\
& (\widetilde{A} \widetilde{B} \widetilde{A})^{1 / 2}=U \operatorname{diag}\left(0_{k_{1}}, C, 0_{k_{3}+k_{4}+k_{5}}, I_{k_{6}}\right) U^{*},
\end{aligned}
$$




$$
\begin{aligned}
& \widetilde{B} \widetilde{A} \widetilde{B}=U \operatorname{diag}\left(\left[\begin{array}{cc}
C^{2} S^{2} & -C^{3} S \\
-C^{3} S & C^{4}
\end{array}\right], 0_{k_{3}+k_{4}+k_{5}}, I_{k_{6}}\right) U^{*}, \\
& (\widetilde{B} \widetilde{A} \widetilde{B})^{1 / 2}=U \operatorname{diag}\left(\left[\begin{array}{cc}
C S^{2} & -C^{2} S \\
-C^{2} S & C^{3}
\end{array}\right], 0_{k_{3}+k_{4}+k_{5}}, I_{k_{6}}\right) U^{*} \text {, } \\
& A B A+B A B=U \operatorname{diag}\left(\left[\begin{array}{cc}
C^{4}+C^{2} & C^{3} S \\
C^{3} S & C^{2} S^{2}
\end{array}\right], 2 I_{k_{3}}, 0_{k_{4}+k_{5}+k_{6}}\right) U^{*} \text {, } \\
& A B A-B A B=U \operatorname{diag}\left(\left[\begin{array}{cc}
C^{2} S^{2} & -C^{3} S \\
-C^{3} S & -C^{2} S^{2}
\end{array}\right], 0_{m-2 k_{1}}\right) U^{*}, \\
& (A B A-B A B)^{2}=U \operatorname{diag}\left(C^{4} S^{2}, C^{4} S^{2}, 0_{m-2 k_{1}}\right) U^{*}, \\
& {\left[(A B A-B A B)^{2}\right]^{1 / 2}=U \operatorname{diag}\left(C^{2} S, C^{2} S, 0_{m-2 k_{1}}\right) U^{*},} \\
& I_{m}-A B A=U \operatorname{diag}\left(S^{2}, I_{k_{2}}, 0_{k_{3}}, I_{k_{4}+k_{5}+k_{6}}\right) U^{*}, \\
& I_{m}-B A B=U \operatorname{diag}\left(\left[\begin{array}{cc}
S^{2}+C^{2} S^{2} & -C^{3} S \\
-C^{3} S & I_{k_{1}}-C^{2} S^{2}
\end{array}\right], 0_{k_{3}}, I_{k_{4}+k_{5}+k_{6}}\right) U^{*}, \\
& 2 I_{m}-A B A-B A B=U \operatorname{diag}\left(\left[\begin{array}{cc}
2 S^{2}+C^{2} S^{2} & -C^{3} S \\
-C^{3} S & 2 I_{k_{2}}-C^{2} S^{2}
\end{array}\right], 0_{k_{3}}, 2 I_{k_{4}+k_{5}+k_{6}}\right) U^{*}, \\
& A-B A B=U \operatorname{diag}\left(\left[\begin{array}{cc}
S^{2}+C^{2} S^{2} & -C^{3} S \\
-C^{3} S & -C^{2} S^{2}
\end{array}\right], 0_{k_{3}}, I_{k_{4}}, 0_{k_{5}+k_{6}}\right) U^{*} \text {, } \\
& B-A B A=U \operatorname{diag}\left(\left[\begin{array}{cc}
0 & C S \\
C S & S^{2}
\end{array}\right], 0_{k_{3}+k_{4}}, I_{k_{5}}, 0_{k_{6}}\right) U^{*} \text {, } \\
& A B A+\widetilde{A} B \widetilde{A}=U \operatorname{diag}\left(C^{2}, S^{2}, I_{k_{3}}, 0_{k_{4}}, I_{k_{5}}, 0_{k_{6}}\right) U^{*} \text {, } \\
& A B \widetilde{A}+\widetilde{A} B A=U \operatorname{diag}\left(\left[\begin{array}{cc}
0 & C S \\
C S & 0
\end{array}\right], 0_{m-2 k_{1}}\right) U^{*}, \\
& B A B+\widetilde{B} A \widetilde{B}=U \operatorname{diag}\left(\left[\begin{array}{cc}
C^{4}+S^{4} & C^{3} S-C S^{3} \\
C^{3} S-C S^{3} & 2 C^{2} S^{2}
\end{array}\right], I_{k_{3}+k_{4}}, 0_{k_{5}+k_{6}}\right) U^{*} \text {, } \\
& B A \widetilde{B}+\widetilde{B} A B=U \operatorname{diag}\left(\left[\begin{array}{cc}
2 C^{2} S^{2} & C S^{3}-C^{3} S \\
C S^{3}-C^{3} S & 2 S^{2} C^{2}
\end{array}\right], 0_{m-2 k_{1}}\right) U^{*} .
\end{aligned}
$$

(b) There exists a unitary matrix $U$ such that the following results hold simultaneously

$$
\begin{aligned}
& (A-B)_{+}=U \operatorname{diag}\left(\frac{1}{2}\left[\begin{array}{cc}
S+S^{2} & -C S \\
-C S & S-S^{2}
\end{array}\right], 0_{k_{3}}, I_{k_{4}}, 0_{k_{5}+k_{6}}\right) U^{*}, \\
& (A-B)_{-}=U \operatorname{diag}\left(\frac{1}{2}\left[\begin{array}{cc}
S^{2}-S & -C S \\
-C S & -S^{2}-S
\end{array}\right], 0_{k_{3}+k_{4}},-I_{k_{5}}, 0_{k_{6}}\right) U^{*}, \\
& \left(A+B-I_{m}\right)_{+}=U \operatorname{diag}\left(\frac{1}{2}\left[\begin{array}{cc}
C+C^{2} & C S \\
C S & C-C^{2}
\end{array}\right], I_{k_{3}}, 0_{k_{4}+k_{5}+k_{6}}\right) U^{*}, \\
& \left(A+B-I_{m}\right)_{-}=U \operatorname{diag}\left(\frac{1}{2}\left[\begin{array}{cc}
C^{2}-C & C S \\
C S & -C^{2}-C
\end{array}\right], 0_{k_{3}+k_{4}+k_{5}},-I_{k_{6}}\right) U^{*}, \\
& (A B+B A)_{+}=U \operatorname{diag}\left(\frac{1}{2}\left[\begin{array}{cc}
C \widehat{C}^{2} & S C \widehat{C} \\
S C \widehat{C} & C S^{2}
\end{array}\right], 2 I_{k_{3}}, 0_{k_{4}+k_{5}+k_{6}}\right) U^{*}, \\
& (A B+B A)_{-}=U \operatorname{diag}\left(\frac{1}{2}\left[\begin{array}{cc}
-C\left(I_{k_{1}}-C\right)^{2} & S C\left(I_{k_{1}}-C\right) \\
S C\left(I_{k_{1}}-C\right) & -C S^{2}
\end{array}\right], 0_{m-2 k_{1}}\right) U^{*},
\end{aligned}
$$




$$
\begin{aligned}
& (A \widetilde{B}+\widetilde{B} A)_{+}=U \operatorname{diag}\left(\frac{1}{2}\left[\begin{array}{cc}
S \widehat{S}^{2} & -S C \widehat{S} \\
-S C \widehat{S} & C^{2} S
\end{array}\right], 0_{k_{3}}, 2 I_{k_{4}}, 0_{k_{5}+k_{6}}\right) U^{*}, \\
& (A \widetilde{B}+\widetilde{B} A)_{-}=U \operatorname{diag}\left(\frac{1}{2}\left[\begin{array}{cc}
-S\left(I_{k_{1}}-S\right)^{2} & -S C\left(I_{k_{1}}-S\right) \\
-S C\left(I_{k_{1}}-S\right) & -C^{2} S
\end{array}\right], 0_{m-2 k_{1}}\right) U^{*}, \\
& (\widetilde{A} B+B \widetilde{A})_{+}=U \operatorname{diag}\left(\frac{1}{2}\left[\begin{array}{cc}
C^{2} S & S C \widehat{S} \\
S C \widehat{S} & S \widehat{S}^{2}
\end{array}\right], 0_{k_{3}+k_{4}}, 2 I_{k_{5}}, 0_{k_{6}}\right) U^{*}, \\
& (\widetilde{A} B+B \widetilde{A})_{-}=U \operatorname{diag}\left(\frac{1}{2}\left[\begin{array}{cc}
-C^{2} S & S C\left(I_{k_{1}}-S\right) \\
S C\left(I_{k_{1}}-S\right) & -S\left(I_{k_{1}}-S\right)^{2}
\end{array}\right], 0_{m-2 k_{1}}\right) U^{*}, \\
& (\widetilde{A} \widetilde{B}+\widetilde{B} \widetilde{A})_{+}=U \operatorname{diag}\left(\frac{1}{2}\left[\begin{array}{cc}
C S^{2} & -S C \widehat{C} \\
-S C \widehat{C} & C \widehat{C}^{2}
\end{array}\right], 0_{k_{3}+k_{4}+k_{5}}, 2 I_{k_{6}}\right) U^{*}, \\
& (\widetilde{A} \widetilde{B}+\widetilde{B} \widetilde{A})_{-}=U \operatorname{diag}\left(\frac{1}{2}\left[\begin{array}{cc}
-C S^{2} & -S C\left(I_{k_{1}}-C\right) \\
-S C\left(I_{k_{1}}-C\right) & -C\left(I_{k_{1}}-C\right)^{2}
\end{array}\right], 0_{m-2 k_{1}}\right) U^{*}, \\
& (A B A-B A B)_{+}=U \operatorname{diag}\left(\frac{1}{2}\left[\begin{array}{cc}
C^{2} S \widehat{S} & -C^{3} S \\
-C^{3} S & C^{2} S\left(I_{k_{1}}-S\right)
\end{array}\right], 0_{m-2 k_{1}}\right) U^{*}, \\
& (A B A-B A B)_{-}=U \operatorname{diag}\left(\frac{1}{2}\left[\begin{array}{cc}
-C^{2} S\left(I_{k_{1}}-S\right) & -C^{3} S \\
-C^{3} S & -C^{2} S \widehat{S}
\end{array}\right], 0_{m-2 k_{1}}\right) U^{*} .
\end{aligned}
$$

(c) The following rank formulas hold

$$
\begin{aligned}
r(A B) & =r(B A)=r(A B A)=r(B A B)=k_{1}+k_{3}, \\
r[A, B] & =r(A+B)=r(A+B-A B)=r(A+B-B A)=2 k_{1}+k_{3}+k_{4}+k_{5}, \\
r\left(A+B-I_{m}\right) & =2 k_{1}+k_{3}+k_{6}, \\
r(A-B) & =r[A \widetilde{B}, B \widetilde{A}]=r[\widetilde{B} A, \widetilde{A} B]=r[A \widetilde{B} A, B \widetilde{A} B]=r[\widetilde{B} A \widetilde{B}, \widetilde{A} B \widetilde{A}] \\
& =r[A-B A B, B-A B A]=r(A \widetilde{B} A+B \widetilde{A} B)=r(\widetilde{B} A \widetilde{B}+\widetilde{A} B \widetilde{A}) \\
& =2 k_{1}+k_{4}+k_{5}, \\
r\left[I_{m}-A, I_{m}-B\right] & =r\left(2 I_{m}-A-B\right)=r\left(I_{m}-A B\right)=r\left(I_{m}-B A\right) \\
& =r\left(I_{m}-A B A\right)=r\left(I_{m}-B A B\right)=2 k_{1}+k_{4}+k_{5}+k_{6}, \\
r[A B, B A] & =r[A B A, B A B]=r(A B+B A)=r(A B A+B A B)=2 k_{1}+k_{3}, \\
r\left[I_{m}-A B, I_{m}-B A\right] & =r\left[I_{m}-A B A, I_{m}-B A B\right]=r\left(2 I_{m}-A B-B A\right) \\
& =r\left(2 I_{m}-A B A-B A B\right)=m-k_{3}, \\
r(A B-B A) & =r(A B A-B A B)=r(A B \widetilde{A}+\widetilde{A} B A)=r(B A \widetilde{B}+\widetilde{B} A B)=2 k_{1}, \\
r(A \widetilde{B}) & =r(\widetilde{B} A)=r(A \widetilde{B} A)=r(\widetilde{B} A \widetilde{B})=k_{1}+k_{4}, \\
r(B \widetilde{A}) & =r(\widetilde{A} B)=r(B \widetilde{A} B)=r(\widetilde{A} B \widetilde{A})=k_{1}+k_{5}, \\
r(\widetilde{A} \widetilde{B} \widetilde{A}) & =r(\widetilde{B} \widetilde{A} \widetilde{B})=k_{1}+k_{6}, \\
r[A \widetilde{B}, \widetilde{B} A] & =r[A \widetilde{B} A, \widetilde{B} A \widetilde{B}]=r(A \widetilde{B}+\widetilde{B} A)=r(A \widetilde{B} A+\widetilde{B} A \widetilde{B})=r(A-B A B) \\
& =2 k_{1}+k_{4}, \\
r[\widetilde{A} B, B \widetilde{A}] & =r[\widetilde{A} B \widetilde{A}, B \widetilde{A} B]=r(\widetilde{A} B+B \widetilde{A})=r(\widetilde{A} B \widetilde{A}+B \widetilde{A} B)=r(B-A B A) \\
r(A B A+\widetilde{A} B \widetilde{A}) & =2 k_{1}+k_{3}+k_{5}, \\
r(B A B+\widetilde{B} A \widetilde{B}) & =2 k_{1}+k_{3}+k_{4}, \\
r[\widetilde{A} \widetilde{B}, \widetilde{B} \widetilde{A}] & =r[\widetilde{A} \widetilde{B} \widetilde{A}, \widetilde{B} \widetilde{A} \widetilde{B}]=r(\widetilde{A} \widetilde{B}+\widetilde{B} \widetilde{A})=r(\widetilde{A} \widetilde{B} \widetilde{A}+\widetilde{B} \widetilde{A} \widetilde{B})=2 k_{1}+k_{6}, \\
r(A B \widetilde{A}) & =r(A \widetilde{B} \widetilde{A})=r(\widetilde{A} B A)=r(\widetilde{A} \widetilde{B} A)=r(B A \widetilde{B})=r(B \widetilde{A} \widetilde{B})=k_{1}, \\
r_{1} &
\end{aligned}
$$




$$
\begin{aligned}
r\left[(A-B)_{+}\right] & =k_{1}+k_{4}, \quad r\left[(A-B)_{-}\right]=k_{1}+k_{5}, \\
r\left[\left(A+B-I_{m}\right)_{+}\right] & =k_{1}+k_{3}, \quad r\left[\left(A+B-I_{m}\right)_{-}\right]=k_{1}+k_{4}, \\
r\left[(A B+B A)_{+}\right] & =k_{1}+k_{3}, r\left[(A B+B A)_{-}\right]=k_{1}, \\
r\left[(A \widetilde{B}+\widetilde{B} A)_{+}\right] & =k_{1}+k_{4}, \quad r\left[(A \widetilde{B}+\widetilde{B} A)_{-}\right]=k_{1}, \\
r\left[(\widetilde{A} B+B \widetilde{A})_{+}\right] & =k_{1}+k_{5}, r\left[(\widetilde{A} B+B \widetilde{A})_{-}\right]=k_{1}, \\
r\left[(\widetilde{A} \widetilde{B}+\widetilde{B} \widetilde{A})_{+}\right] & =k_{1}+k_{6}, \quad r\left[(\widetilde{A} \widetilde{B}+\widetilde{B} \widetilde{A})_{-}\right]=k_{1}, \\
r\left[(A B A-B A B)_{+}\right] & =r\left[(A B A-B A B)_{-}\right]=k_{1} .
\end{aligned}
$$

(d) There exists a unitary matrix $U$ such that

$$
\begin{aligned}
& (A+B)^{\dagger}=U \operatorname{diag}\left(\left[\begin{array}{cc}
I_{k_{1}} & -C S^{-1} \\
-C S^{-1} & S^{-2}\left(I_{k_{1}}+C^{2}\right)
\end{array}\right], 2^{-1} I_{k_{3}}, I_{k_{4}+k_{5}}, 0_{k_{6}}\right) U^{*}, \\
& \left(A+B-I_{m}\right)^{\dagger}=U \operatorname{diag}\left(\left[\begin{array}{cc}
I_{k_{1}} & C^{-1} S \\
C^{-1} S & -I_{k_{1}}
\end{array}\right], I_{k_{3}}, 0_{k_{4}+k_{5}},-I_{k_{6}}\right) U^{*}, \\
& (A-B)^{\dagger}=U \operatorname{diag}\left(\left[\begin{array}{cc}
I_{k_{1}} & -C S^{-1} \\
-C S^{-1} & -I_{k_{1}}
\end{array}\right], 0_{k_{3}}, I_{k_{4}},-I_{k_{5}}, 0_{k_{6}}\right) U^{*} \text {, } \\
& \left(2 I_{m}-A-B\right)^{\dagger}=U \operatorname{diag}\left(\left[\begin{array}{cc}
S^{-2}\left(I_{k_{1}}+C^{2}\right) & C S^{-1} \\
C S^{-1} & I_{k_{1}}
\end{array}\right], 0_{k_{3}}, I_{k_{4}+k_{5}}, 2^{-1} I_{k_{6}}\right) U^{*}, \\
& (A B)^{\dagger}=U \operatorname{diag}\left(\left[\begin{array}{cc}
I_{k_{1}} & 0 \\
C^{-1} S & 0
\end{array}\right], I_{k_{3}}, 0_{k_{4}+k_{5}+k_{6}}\right) U^{*}, \\
& (B A)^{\dagger}=U \operatorname{diag}\left(\left[\begin{array}{cc}
I_{k_{1}} & C^{-1} S \\
0 & 0
\end{array}\right], I_{k_{3}}, 0_{k_{4}+k_{5}+k_{6}}\right) U^{*}, \\
& (A+B-A B)^{\dagger}=U \operatorname{diag}\left(\left[\begin{array}{cc}
I_{k_{1}} & 0 \\
-C S^{-1} & S^{-2}
\end{array}\right], I_{k_{3}+k_{4}+k_{5}}, 0_{k_{6}}\right) U^{*}, \\
& (A+B-B A)^{\dagger}=U \operatorname{diag}\left(\left[\begin{array}{cc}
I_{k_{1}} & -C S^{-1} \\
0 & S^{-2}
\end{array}\right], I_{k_{3}+k_{4}+k_{5}}, 0_{k_{6}}\right) U^{*}, \\
& (A B+B A)^{\dagger}=U \operatorname{diag}\left(\left[\begin{array}{cc}
0 & (C S)^{-1} \\
(C S)^{-1} & -2 S^{-2}
\end{array}\right], 2^{-1} I_{k_{3}}, 0_{k_{4}+k_{5}+k_{6}}\right) U^{*}, \\
& (A B-B A)^{\dagger}=U \operatorname{diag}\left(\left[\begin{array}{cc}
0 & -(C S)^{-1} \\
(C S)^{-1} & 0
\end{array}\right], 0_{m-2 k_{1}}\right) U^{*}, \\
& \left(I_{m}-A B\right)^{\dagger}=U \operatorname{diag}\left(\left[\begin{array}{cc}
S^{-2} & C S^{-1} \\
0 & I_{k_{1}}
\end{array}\right], 0_{k_{3}}, I_{k_{4}+k_{5}+k_{6}}\right) U^{*}, \\
& \left(I_{m}-B A\right)^{\dagger}=U \operatorname{diag}\left(\left[\begin{array}{cc}
S^{-2} & 0 \\
C S^{-1} & I_{k_{1}}
\end{array}\right], 0_{k_{3}}, I_{k_{4}+k_{5}+k_{6}}\right) U^{*} \text {, } \\
& (A \widetilde{B})^{\dagger}=U \operatorname{diag}\left(\left[\begin{array}{cc}
I_{k_{1}} & 0 \\
-C S^{-1} & 0
\end{array}\right], 0_{k_{3}}, I_{k_{4}}, 0_{k_{5}+k_{6}}\right) U^{*}, \\
& (\widetilde{B} A)^{\dagger}=U \operatorname{diag}\left(\left[\begin{array}{cc}
I_{k_{1}} & -C S^{-1} \\
0 & 0
\end{array}\right], 0_{k_{3}}, I_{k_{4}}, 0_{k_{5}+k_{6}}\right) U^{*}, \\
& (B \widetilde{A})^{\dagger}=U \operatorname{diag}\left(\left[\begin{array}{cc}
0 & 0 \\
C S^{-1} & I_{k_{1}}
\end{array}\right], 0_{k_{3}+k_{4}}, I_{k_{5}}, 0_{k_{6}}\right) U^{*}, \\
& (\widetilde{A} B)^{\dagger}=U \operatorname{diag}\left(\left[\begin{array}{cc}
0 & C S^{-1} \\
0 & I_{k_{1}}
\end{array}\right], 0_{k_{3}+k_{4}}, I_{k_{5}}, 0_{k_{6}}\right) U^{*},
\end{aligned}
$$




$$
\begin{aligned}
& (\widetilde{A} \widetilde{B})^{\dagger}=U \operatorname{diag}\left(\left[\begin{array}{cc}
0 & -C^{-1} S \\
0 & I_{k_{1}}
\end{array}\right], 0_{k_{3}+k_{4}+k_{5}}, I_{k_{6}}\right) U^{*}, \\
& (\widetilde{B} \widetilde{A})^{\dagger}=U \operatorname{diag}\left(\left[\begin{array}{cc}
0 & 0 \\
-C^{-1} S & I_{k_{1}}
\end{array}\right], 0_{k_{3}+k_{4}+k_{5}}, I_{k_{6}}\right) U^{*}, \\
& (A \widetilde{B}+\widetilde{B} A)^{\dagger}=U \operatorname{diag}\left(\left[\begin{array}{cc}
0 & -(C S)^{-1} \\
-(C S)^{-1} & -2 C^{-2}
\end{array}\right], 0_{k_{3}}, 2^{-1} I_{k_{4}}, 0_{k_{5}+k_{6}}\right) U^{*} \text {, } \\
& (\widetilde{A} B+B \widetilde{A})^{\dagger}=U \operatorname{diag}\left(\left[\begin{array}{cc}
-2 C^{-2} & (C S)^{-1} \\
(C S)^{-1} & 0
\end{array}\right], 0_{k_{3}+k_{4}}, 2^{-1} I_{k_{5}}, 0_{k_{6}}\right) U^{*}, \\
& (\widetilde{A} \widetilde{B}+\widetilde{B} \widetilde{A})^{\dagger}=U \operatorname{diag}\left(\left[\begin{array}{cc}
-2 S^{-2} & -(C S)^{-1} \\
-(C S)^{-1} & 0
\end{array}\right], 0_{k_{3}+k_{4}+k_{5}}, 2^{-1} I_{k_{6}}\right) U^{*}, \\
& (A B A)^{\dagger}=U \operatorname{diag}\left(C^{-2}, 0_{k_{2}}, I_{k_{3}}, 0_{k_{4}+k_{5}+k_{6}}\right) U^{*} \text {, } \\
& (B A B)^{\dagger}=U \operatorname{diag}\left(\left[\begin{array}{cc}
I_{k_{1}} & C^{-1} S \\
C^{-1} S & C^{-2} S^{2}
\end{array}\right], I_{k_{3}}, 0_{k_{4}+k_{5}+k_{6}}\right) U^{*}, \\
& (A B \widetilde{A})^{\dagger}=-(A \widetilde{B} \widetilde{A})^{\dagger}=U \operatorname{diag}\left(\left[\begin{array}{cc}
0 & 0 \\
(C S)^{-1} & 0
\end{array}\right], 0_{m-2 k_{1}}\right) U^{*} \text {, } \\
& (\widetilde{A} B A)^{\dagger}=-(\widetilde{A} \widetilde{B} A)^{\dagger}=U \operatorname{diag}\left(\left[\begin{array}{cc}
0 & (C S)^{-1} \\
0 & 0
\end{array}\right], 0_{m-2 k_{1}}\right) U^{*}, \\
& (B A \widetilde{B})^{\dagger}=-(B \widetilde{A} \widetilde{B})^{\dagger}=U \operatorname{diag}\left(\left[\begin{array}{cc}
I_{k_{1}} & C^{-1} S \\
-C S^{-1} & -I_{k_{1}}
\end{array}\right], 0_{m-2 k_{1}}\right) U^{*}, \\
& (\widetilde{B} A B)^{\dagger}=-(\widetilde{B} \widetilde{A} B)^{\dagger}=U \operatorname{diag}\left(\left[\begin{array}{cc}
I_{k_{1}} & -C S^{-1} \\
C^{-1} S & -I_{k_{1}}
\end{array}\right], 0_{m-2 k_{1}}\right) U^{*} \text {, } \\
& (A \widetilde{B} A)^{\dagger}=U \operatorname{diag}\left(S^{-2}, 0_{k_{2}+k_{3}}, I_{k_{4}}, 0_{k_{5}+k_{6}}\right) U^{*} \text {, } \\
& (B \widetilde{A} B)^{\dagger}=U \operatorname{diag}\left(\left[\begin{array}{cc}
C^{2} S^{-2} & C S^{-1} \\
C S^{-1} & I_{k_{1}}
\end{array}\right], 0_{m-2 k_{1}}\right) U^{*}, \\
& (\widetilde{A} B \widetilde{A})^{\dagger}=U \operatorname{diag}\left(0_{k_{1}}, S^{-2}, 0_{k_{3}+k_{4}}, I_{k_{5}}, 0_{k_{6}}\right) U^{*} \text {, } \\
& (\widetilde{B} A \widetilde{B})^{\dagger}=U \operatorname{diag}\left(\left[\begin{array}{cc}
I_{k_{1}} & -C S^{-1} \\
-C S^{-1} & C^{2} S^{-2}
\end{array}\right], 0_{k_{3}}, I_{k_{4}}, 0_{k_{5}+k_{6}}\right) U^{*}, \\
& (\widetilde{A} \widetilde{B} \widetilde{A})^{\dagger}=U \operatorname{diag}\left(0_{k_{1}}, C^{-2}, 0_{k_{3}+k_{4}+k_{5}}, I_{k_{6}}\right) U^{*} \text {, } \\
& (\widetilde{B} \widetilde{A} \widetilde{B})^{\dagger}=U \operatorname{diag}\left(\left[\begin{array}{cc}
C^{-2} S^{2} & -C^{-1} S \\
-C^{-1} S & I_{k_{1}}
\end{array}\right], 0_{k_{3}+k_{4}+k_{5}}, I_{k_{6}}\right) U^{*}, \\
& (A B A+B A B)^{\dagger}=U \operatorname{diag}\left(\left[\begin{array}{cc}
C^{-2} & -(C S)^{-1} \\
-(C S)^{-1} & S^{-2}+(C S)^{-2}
\end{array}\right], 2^{-1} I_{k_{3}}, 0_{k_{4}+k_{5}+k_{6}}\right) U^{*} \text {, } \\
& (A B A-B A B)^{\dagger}=U \operatorname{diag}\left(\left[\begin{array}{cc}
C^{-2} & -(C S)^{-1} \\
-(C S)^{-1} & -C^{-2}
\end{array}\right], 0_{m-2 k_{1}}\right) U^{*}, \\
& \left(I_{m}-A B A\right)^{\dagger}=U \operatorname{diag}\left(S^{-2}, I_{k_{1}}, 0_{k_{3}}, I_{k_{4}+k_{5}+k_{6}}\right) U^{*} \text {, } \\
& \left(I_{m}-B A B\right)^{\dagger}=U \operatorname{diag}\left(\left[\begin{array}{cc}
S^{-2}-C^{2} & C^{3} S^{-1} \\
C^{3} S^{-1} & I_{k_{1}}+C^{2}
\end{array}\right], 0_{k_{3}}, I_{k_{4}+k_{5}+k_{6}}\right) U^{*} \text {, } \\
& \left(2 I_{m}-A B A-B A B\right)^{\dagger}=U \operatorname{diag}\left(\left[\begin{array}{cc}
2 S^{2}+C^{2} S^{2} & -C^{3} S \\
-C^{3} S & 2 I_{k_{2}}-C^{2} S^{2}
\end{array}\right]^{-1}, 0_{k_{3}}, 2^{-1} I_{k_{4}+k_{5}+k_{6}}\right) U^{*} \text {, }
\end{aligned}
$$




$$
\begin{aligned}
& (A-B A B)^{\dagger}=U \operatorname{diag}\left(\left[\begin{array}{cc}
I_{k_{1}} & -C S^{-1} \\
-C S^{-1} & -I_{k_{1}}-C^{-2}
\end{array}\right], 0_{k_{3}}, I_{k_{4}}, 0_{k_{5}+k_{6}}\right) U^{*}, \\
& (B-A B A)^{\dagger}=U \operatorname{diag}\left(\left[\begin{array}{cc}
-C^{-2} & (C S)^{-1} \\
(C S)^{-1} & 0
\end{array}\right], 0_{k_{3}+k_{4}}, I_{k_{5}}, 0_{k_{6}}\right) U^{*} \text {, } \\
& (A B A+\widetilde{A} B \widetilde{A})^{\dagger}=U \operatorname{diag}\left(C^{-2}, S^{-2}, I_{k_{3}}, 0_{k_{4}}, I_{k_{5}}, 0_{k_{6}}\right) U^{*}, \\
& (A B \tilde{A}+\widetilde{A} B A)^{\dagger}=U \operatorname{diag}\left(\left[\begin{array}{cc}
0 & (C S)^{-1} \\
(C S)^{-1} & 0
\end{array}\right], 0_{m-2 k_{1}}\right) U^{*}, \\
& (B A B+\widetilde{B} A \widetilde{B})^{\dagger}=U \operatorname{diag}\left(\left[\begin{array}{cc}
2 I_{k_{1}} & C^{-1} S-C S^{-1} \\
C^{-1} S-C S^{-1} & C^{2} S^{-2}+C^{-2} S^{2}
\end{array}\right], I_{k_{3}+k_{4}}, 0_{k_{5}+k_{6}}\right) U^{*}, \\
& (B A \widetilde{B}+\widetilde{B} A B)^{\dagger}=U \operatorname{diag}\left(\left[\begin{array}{cc}
2 I_{k_{1}} & C^{-1} S-C S^{-1} \\
C^{-1} S-C S^{-1} & -2 I_{k_{1}}
\end{array}\right], 0_{m-2 k_{1}}\right) U^{*}, \\
& (A-B)_{+}^{\dagger}=U \operatorname{diag}\left(\frac{1}{2}\left[\begin{array}{cc}
I_{k_{1}}+S^{-1} & -C S^{-1} \\
-C S^{-1} & S^{-1}-I_{k_{1}}
\end{array}\right], 0_{k_{3}}, I_{k_{4}}, 0_{k_{5}+k_{6}}\right) U^{*} \text {, } \\
& (A-B)_{-}^{\dagger}=U \operatorname{diag}\left(\frac{1}{2}\left[\begin{array}{cc}
I_{k_{1}}-S^{-1} & -C S^{-1} \\
-C S^{-1} & -I_{k_{1}}-S^{-1}
\end{array}\right], 0_{k_{3}+k_{4}},-I_{k_{5}}, 0_{k_{6}}\right) U^{*}, \\
& \left(A+B-I_{m}\right)_{+}^{\dagger}=U \operatorname{diag}\left(\frac{1}{2}\left[\begin{array}{cc}
I_{k_{1}}+C^{-1} & C^{-1} S \\
C^{-1} S & C^{-1}-I_{k_{1}}
\end{array}\right], I_{k_{3}}, 0_{k_{4}+k_{5}+k_{6}}\right) U^{*}, \\
& \left(A+B-I_{m}\right)_{-}^{\dagger}=U \operatorname{diag}\left(\frac{1}{2}\left[\begin{array}{cc}
I_{k_{1}}-C^{-1} & C^{-1} S \\
C^{-1} S & -I_{k_{1}}-C^{-1}
\end{array}\right], 0_{k_{3}+k_{4}+k_{5}},-I_{k_{6}}\right) U^{*}, \\
& (A B+B A)_{+}^{\dagger}=U \operatorname{diag}\left(\frac{1}{2}\left[\begin{array}{cc}
C^{-1} & (C S)^{-1}\left(I_{k_{1}}-C\right) \\
(C S)^{-1}\left(I_{k_{1}}-C\right) & C^{-1} S^{-2}\left(I_{k_{1}}-C\right)^{2}
\end{array}\right], 2^{-1} I_{k_{3}}, 0_{k_{4}+k_{5}+k_{6}}\right) U^{*}, \\
& (A B+B A)_{-}^{\dagger}=U \operatorname{diag}\left(\frac{1}{2}\left[\begin{array}{cc}
-C^{-1} & (C S)^{-1} \widehat{C} \\
(C S)^{-1} \widehat{C} & -C^{-1} S^{-2} \widehat{C}^{2}
\end{array}\right], 0_{k_{3}+k_{4}+k_{5}+k_{6}}\right) U^{*}, \\
& (A \widetilde{B}+\widetilde{B} A)_{+}^{\dagger}=U \operatorname{diag}\left(\frac{1}{2}\left[\begin{array}{cc}
S^{-1} & -(C S)^{-1}\left(I_{k_{1}}-S\right) \\
-(C S)^{-1}\left(I_{k_{1}}-S\right) & C^{-2} S^{-1}\left(I_{k_{1}}-S\right)^{2}
\end{array}\right], 0_{k_{3}}, 2^{-1} I_{k_{4}}, 0_{k_{5}+k_{6}}\right) U^{*}, \\
& (A \widetilde{B}+\widetilde{B} A)_{-}^{\dagger}=U \operatorname{diag}\left(\frac{1}{2}\left[\begin{array}{cc}
-S^{-1} & -(C S)^{-1} \widehat{S} \\
-(C S)^{-1} \widehat{S} & -C^{-2} S^{-1} \widehat{S}^{2}
\end{array}\right], 0_{m-2 k_{1}}\right) U^{*}, \\
& (\widetilde{A} B+B \widetilde{A})_{+}^{\dagger}=U \operatorname{diag}\left(\frac{1}{2}\left[\begin{array}{cc}
C^{-2} S^{-1}\left(I_{k_{1}}-S\right)^{2} & (C S)^{-1}\left(I_{k_{1}}-S\right) \\
(C S)^{-1}\left(I_{k_{1}}-S\right) & S^{-1}
\end{array}\right], 0_{k_{3}+k_{4}}, 2^{-1} I_{k_{5}}, 0_{k_{6}}\right) U^{*}, \\
& (\tilde{A} B+B \widetilde{A})_{-}^{\dagger}=U \operatorname{diag}\left(\frac{1}{2}\left[\begin{array}{cc}
-C^{-2} S^{-1}\left(I_{k_{1}}+S\right)^{2} & (C S)^{-1}\left(I_{k_{1}}-S\right) \\
(C S)^{-1}\left(I_{k_{1}}-S\right) & -S^{-1}
\end{array}\right], 0_{m-2 k_{1}}\right) U^{*}, \\
& (\widetilde{A} \widetilde{B}+\widetilde{B} \widetilde{A})_{+}^{\dagger}=U \operatorname{diag}\left(\frac{1}{2}\left[\begin{array}{cc}
C^{-1} S^{-2}\left(I_{k_{1}}-C\right)^{2} & -(C S)^{-1}\left(I_{k_{1}}-C\right) \\
-(C S)^{-1}\left(I_{k_{1}}-C\right) & C^{-1}
\end{array}\right], 0_{k_{3}+k_{4}+k_{5}}, 2^{-1} I_{k_{6}}\right) U^{*}, \\
& (\widetilde{A} \widetilde{B}+\widetilde{B} \widetilde{A})_{-}^{\dagger}=U \operatorname{diag}\left(\frac{1}{2}\left[\begin{array}{cc}
-C^{-1} S^{-2}\left(I_{k_{1}}+C\right)^{2} & -(C S)^{-1} \widehat{C} \\
-(C S)^{-1} \widehat{C} & -C^{-1}
\end{array}\right], 0_{m-2 k_{1}}\right) U^{*}, \\
& (A B A-B A B)_{+}^{\dagger}=U \operatorname{diag}\left(\frac{1}{2}\left[\begin{array}{cc}
C^{-2} S^{-1} \widehat{S} & -(C S)^{-1} \\
-(C S)^{-1} & C^{-2} S^{-1}\left(I_{k_{1}}-S\right)
\end{array}\right], 0_{m-2 k_{1}}\right) U^{*}, \\
& (A B A-B A B)_{-}^{\dagger}=U \operatorname{diag}\left(\frac{1}{2}\left[\begin{array}{cc}
-C^{-2} S^{-1}\left(I_{k_{1}}-S\right) & -(C S)^{-1} \\
-(C S)^{-1} & -C^{-2} S^{-1} \widehat{S}
\end{array}\right], 0_{m-2 k_{1}}\right) U^{*}, \\
& (A+B)(A+B)^{\dagger}=U \operatorname{diag}\left(I_{2 k_{1}}, 0_{m-2 k_{1}}\right) U^{*} \text {, }
\end{aligned}
$$




$$
\begin{aligned}
& \left(I_{m}+A-B\right)\left(I_{m}+A-B\right)^{\dagger}=U \operatorname{diag}\left(I_{2 k_{1}+k_{3}+k_{4}}, 0_{k_{5}}, I_{k_{6}}\right) U^{*}, \\
& \left(I_{m}-A-B\right)\left(I_{m}-A-B\right)^{\dagger}=U \operatorname{diag}\left(I_{2 k_{1}+k_{3}}, 0_{k_{4}+k_{5}}, I_{k_{6}}\right) U^{*} \text {, } \\
& (A-B)(A-B)^{\dagger}=U \operatorname{diag}\left(I_{2 k_{1}} 0_{k_{3}}, I_{k_{4}+k_{5}}, 0_{k_{6}}\right) U^{*} \text {, } \\
& \left(2 I_{m}-A-B\right)\left(2 I_{m}-A-B\right)^{\dagger}=U \operatorname{diag}\left(I_{2 k_{1}}, 0_{k_{3}}, I_{k_{4}+k_{5}+k_{6}}\right) U^{*}, \\
& (A B)(A B)^{\dagger}=(A B A)(A B A)^{\dagger}=U \operatorname{diag}\left(I_{k_{1}}, 0_{k_{2}}, I_{k_{3}}, 0_{k_{4}+k_{5}+k_{6}}\right) U^{*} \text {, } \\
& (B A)(B A)^{\dagger}=(B A B)(B A B)^{\dagger}=U \operatorname{diag}\left(\left[\begin{array}{cc}
C^{2} & C S \\
C S & S^{2}
\end{array}\right], I_{k_{3}}, 0_{k_{4}+k_{5}+k_{6}}\right) U^{*} \text {, } \\
& (A+B-A B)(A+B-A B)^{\dagger}=U \operatorname{diag}\left(I_{m-k_{6}}, 0_{k_{6}}\right) U^{*}, \\
& (A+B-B A)(A+B-B A)^{\dagger}=U \operatorname{diag}\left(I_{m-k_{6}}, 0_{k_{6}}\right) U^{*}, \\
& (A B+B A)(A B+B A)^{\dagger}=U \operatorname{diag}\left(I_{2 k_{1}+k_{3}}, 0_{k_{4}+k_{5}+k_{6}}\right) U^{*}, \\
& \left(2 I_{m}-A B-B A\right)\left(2 I_{m}-A B-B A\right)^{\dagger}=U \operatorname{diag}\left(I_{2 k_{1}}, 0_{k_{3}}, I_{k_{4}+k_{5}+k_{6}}\right) U^{*}, \\
& (A B-B A)(A B-B A)^{\dagger}=U \operatorname{diag}\left(I_{2 k_{1}}, 0_{m-2 k_{1}}\right) U^{*}, \\
& \left(I_{m}-A B\right)\left(I_{m}-A B\right)^{\dagger}=U \operatorname{diag}\left(I_{2 k_{1}}, 0_{k_{3}}, I_{k_{4}+k_{5}+k_{6}}\right) U^{*}, \\
& \left(I_{m}-B A\right)\left(I_{m}-B A\right)^{\dagger}=U \operatorname{diag}\left(I_{2 k_{1}}, 0_{k_{3}}, I_{k_{4}+k_{5}+k_{6}}\right) U^{*}, \\
& (A \widetilde{B})(A \widetilde{B})^{\dagger}=(A \widetilde{B} A)(A \widetilde{B} A)^{\dagger}=U \operatorname{diag}\left(I_{k_{1}}, 0_{k_{2}+k_{3}}, I_{k_{4}}, 0_{k_{5}+k_{6}}\right) U^{*} \text {, } \\
& (\widetilde{B} A)(\widetilde{B} A)^{\dagger}=(\widetilde{B} A \widetilde{B})(\widetilde{B} A \widetilde{B})^{\dagger}=U \operatorname{diag}\left(\left[\begin{array}{cc}
S^{2} & -C S \\
-C S & C^{2}
\end{array}\right], 0_{k_{3}}, I_{k_{4}}, 0_{k_{5}+k_{6}}\right) U^{*}, \\
& (B \widetilde{A})(B \widetilde{A})^{\dagger}=(B \widetilde{A} B)(B \widetilde{A} B)^{\dagger}=U \operatorname{diag}\left(\left[\begin{array}{cc}
C^{2} & C S \\
C S & S^{2}
\end{array}\right], 0_{k_{3}+k_{4}}, I_{k_{5}}, 0_{k_{6}}\right) U^{*}, \\
& (\widetilde{A} B)(\widetilde{A} B)^{\dagger}=(\widetilde{A} B \widetilde{A})(\widetilde{A} B \widetilde{A})^{\dagger}=U \operatorname{diag}\left(0_{k_{1}}, I_{k_{2}}, 0_{k_{3}+k_{4}}, I_{k_{5}}, 0_{k_{6}}\right) U^{*}, \\
& (\widetilde{A} \widetilde{B})(\widetilde{A} \widetilde{B})^{\dagger}=(\widetilde{A} \widetilde{B} \widetilde{A})(\widetilde{A} \widetilde{B} \widetilde{A})^{\dagger}=U \operatorname{diag}\left(0_{k_{1}}, I_{k_{2}}, 0_{k_{3}+k_{4}+k_{5}}, I_{k_{6}}\right) U^{*} \text {, } \\
& (\widetilde{B} \widetilde{A})(\widetilde{B} \widetilde{A})^{\dagger}=(\widetilde{B} \widetilde{A} \widetilde{B})(\widetilde{B} \widetilde{A} \widetilde{B})^{\dagger}=U \operatorname{diag}\left(\left[\begin{array}{cc}
S^{2} & -C S \\
-C S & C^{2}
\end{array}\right], 0_{k_{3}+k_{4}+k_{5}}, I_{k_{6}}\right) U^{*}, \\
& (A \widetilde{B}+\widetilde{B} A)(A \widetilde{B}+\widetilde{B} A)^{\dagger}=U \operatorname{diag}\left(I_{2 k_{1}}, 0_{k_{3}}, I_{k_{4}}, 0_{k_{5}+k_{6}}\right) U^{*}, \\
& (\widetilde{A} B+B \widetilde{A})(\widetilde{A} B+B \widetilde{A})^{\dagger}=U \operatorname{diag}\left(I_{2 k_{1}}, 0_{k_{3}+k_{4}}, I_{k_{5}}, 0_{k_{6}}\right) U^{*}, \\
& (\widetilde{A} \widetilde{B}+\widetilde{B} \widetilde{A})(\widetilde{A} \widetilde{B}+\widetilde{B} \widetilde{A})^{\dagger}=U \operatorname{diag}\left(I_{2 k_{1}}, 0_{k_{3}+k_{4}+k_{5}}, I_{k_{6}}\right) U^{*}, \\
& (A B \widetilde{A})(A B \widetilde{A})^{\dagger}=(A \widetilde{B} \widetilde{A})(A \widetilde{B} \widetilde{A})^{\dagger}=U \operatorname{diag}\left(I_{k_{1}}, 0_{m-k_{1}}\right) U^{*}, \\
& (\widetilde{A} B A)(\widetilde{A} B A)^{\dagger}=(\widetilde{A} \widetilde{B} A)(\widetilde{A} \widetilde{B} A)^{\dagger}=U \operatorname{diag}\left(0_{k_{1}}, I_{k_{1}}, 0_{m-2 k_{1}}\right) U^{*}, \\
& (B A \widetilde{B})(B A \widetilde{B})^{\dagger}=(B \widetilde{A} \widetilde{B})(B \widetilde{A} \widetilde{B})^{\dagger}=U \operatorname{diag}\left(\left[\begin{array}{cc}
C^{2} & C S \\
C S & S^{2}
\end{array}\right], 0_{m-2 k_{1}}\right) U^{*}, \\
& (\widetilde{B} A B)(\widetilde{B} A B)^{\dagger}=(\widetilde{B} \widetilde{A} B)(\widetilde{B} \widetilde{A} B)^{\dagger}=U \operatorname{diag}\left(\left[\begin{array}{cc}
S^{2} & -C S \\
-C S & C^{2}
\end{array}\right], 0_{m-2 k_{1}}\right) U^{*}, \\
& (A B A+B A B)(A B A+B A B)^{\dagger}=U \operatorname{diag}\left(I_{2 k_{1}+k_{3}}, 0_{k_{4}+k_{5}+k_{6}}\right) U^{*}, \\
& (A B A-B A B)(A B A-B A B)^{\dagger}=U \operatorname{diag}\left(I_{2 k_{1}}, 0_{m-2 k_{1}}\right) U^{*} \text {, } \\
& \left(I_{m}-A B A\right)\left(I_{m}-A B A\right)^{\dagger}=U \operatorname{diag}\left(I_{2 k_{1}}, 0_{k_{3}}, I_{k_{4}+k_{5}+k_{6}}\right) U^{*}, \\
& \left(I_{m}-B A B\right)\left(I_{m}-B A B\right)^{\dagger}=U \operatorname{diag}\left(I_{2 k_{1}}, 0_{k_{3}}, I_{k_{4}+k_{5}+k_{6}}\right) U^{*} \text {, } \\
& \left(2 I_{m}-A B A-B A B\right)\left(2 I_{m}-A B A-B A B\right)^{\dagger}=U \operatorname{diag}\left(I_{2 k_{1}}, 0_{k_{3}}, I_{k_{4}+k_{5}+k_{6}}\right) U^{*} \text {, } \\
& (A-B A B)(A-B A B)^{\dagger}=U \operatorname{diag}\left(I_{2 k_{1}}, 0_{k_{3}}, I_{k_{4}}, 0_{k_{5}+k_{6}}\right) U^{*} \text {, } \\
& (B-A B A)(B-A B A)^{\dagger}=U \operatorname{diag}\left(I_{2 k_{1}}, 0_{k_{3}+k_{4}}, I_{k_{5}}, 0_{k_{6}}\right) U^{*} \text {, }
\end{aligned}
$$




$$
\begin{aligned}
& (A B A+\widetilde{A} B \widetilde{A})(A B A+\widetilde{A} B \widetilde{A})^{\dagger}=U \operatorname{diag}\left(I_{2 k_{1}+k_{3}}, 0_{k_{4}}, I_{k_{5}}, 0_{k_{6}}\right) U^{*}, \\
& (A B \widetilde{A}+\widetilde{A} B A)(A B \widetilde{A}+\widetilde{A} B A)^{\dagger}=U \operatorname{diag}\left(I_{2 k_{1}}, 0_{m-2 k_{1}}\right) U^{*}, \\
& (B A B+\widetilde{B} \widetilde{B})(B A B+\widetilde{B} A \widetilde{B})^{\dagger}=U \operatorname{diag}\left(I_{2 k_{1}+k_{3}+k_{4}}, 0_{k_{5}+k_{6}}\right) U^{*}, \\
& (B A \widetilde{B}+\widetilde{B} A B)(B A \widetilde{B}+\widetilde{B} A B)^{\dagger}=U \operatorname{diag}\left(I_{2 k_{1}}, 0_{m-2 k_{1}}\right) U^{*}, \\
& (A B)^{\#}=U \operatorname{diag}\left(\left[\begin{array}{cc}
C^{-2} & C^{-3} S \\
0 & 0
\end{array}\right], I_{k_{3}}, 0_{m-2 k_{1}-k_{3}}\right) U^{*}, \\
& (B A)^{\#}=U \operatorname{diag}\left(\left[\begin{array}{cc}
C^{-2} & 0 \\
C^{-3} S & 0
\end{array}\right], I_{k_{3}}, 0_{m-2 k_{1}-k_{3}}\right) U^{*}, \\
& (A B)(A B)^{\#}=U \operatorname{diag}\left(\left[\begin{array}{cc}
I_{k_{1}} & C^{-1} S \\
0 & 0
\end{array}\right], I_{k_{3}}, 0_{m-2 k_{1}-k_{3}}\right) U^{*}, \\
& (B A)(B A)^{\#}=U \operatorname{diag}\left(\left[\begin{array}{cc}
I_{k_{1}} & 0 \\
C^{-1} S & 0
\end{array}\right], I_{k_{3}}, 0_{m-2 k_{1}-k_{3}}\right) U^{*}, \\
& (A B)^{\#}+(B A)^{\#}=U \operatorname{diag}\left(\left[\begin{array}{cc}
2 C^{-2} & C^{-3} S \\
C^{-3} S & 0
\end{array}\right], 2 I_{k_{3}}, 0_{m-2 k_{1}-k_{3}}\right) U^{*} .
\end{aligned}
$$

(e) The orthogonal projectors onto $\mathcal{M} \cap \mathcal{N}, \mathcal{M} \cap \mathcal{N}^{\perp}, \mathcal{M}^{\perp} \cap \mathcal{N}$, and $\mathcal{M}^{\perp} \cap \mathcal{N}^{\perp}$ can simultaneously be decomposed as

$$
\begin{array}{ll}
P_{\mathcal{M} \cap \mathcal{N}}=U \operatorname{diag}\left(0_{2 k_{1}}, I_{k_{3}}, 0_{k_{4}+k_{5}+k_{6}}\right) U^{*}, & P_{\mathcal{N} \cap \mathcal{N}^{\perp}}=U \operatorname{diag}\left(0_{2 k_{1}+k_{3}}, I_{k_{4}}, 0_{k_{5}+k_{6}}\right) U^{*}, \\
P_{\mathcal{M M}^{\perp} \cap \mathcal{N}}=U \operatorname{diag}\left(0_{2 k_{1}+k_{3}+k_{4}}, I_{k_{5}}, 0_{k_{6}}\right) U^{*}, & P_{\mathcal{M}^{\perp} \cap \mathcal{N}^{\perp}}=U \operatorname{diag}\left(0_{m-k_{6}}, I_{k_{6}}\right) U^{*},
\end{array}
$$

and the dimensions of $\mathcal{M} \cap \mathcal{N}, \mathcal{M} \cap \mathcal{N}^{\perp}, \mathcal{M}^{\perp} \cap \mathcal{N}$, and $\mathcal{M}^{\perp} \cap \mathcal{N}^{\perp}$ are

$$
\begin{aligned}
& \operatorname{dim}(\mathcal{M} \cap \mathcal{N})=r(A)+r(B)-r[A, B], \quad \operatorname{dim}\left(\mathcal{M} \cap \mathcal{N}^{\perp}\right)=r(A)-r(A B), \\
& \operatorname{dim}\left(\mathcal{M}^{\perp} \cap \mathcal{N}\right)=r(B)-r(A B), \quad \operatorname{dim}\left(\mathcal{M}^{\perp} \cap \mathcal{N}^{\perp}\right)=m-r[A, B] .
\end{aligned}
$$

(f) The following results hold

$$
\begin{aligned}
& A B=B A \Leftrightarrow A B A=B A B \Leftrightarrow A B \widetilde{A}+\widetilde{A} B A=0 \Leftrightarrow B A \widetilde{B}+\widetilde{B} A B=0 \\
& \Leftrightarrow r[A, B]=r(A)+r(B)-r(A B) \Leftrightarrow k_{1}=k_{2}=0, \\
& \mathcal{M} \cap \mathcal{N}=\{0\} \Leftrightarrow r[A, B]=r(A)+r(B) \Leftrightarrow k_{3}=0, \\
& \mathcal{M} \cap \mathcal{N}^{\perp}=\{0\} \Leftrightarrow r(A)=r(A B) \Leftrightarrow k_{4}=0, \\
& \mathcal{M}^{\perp} \cap \mathcal{N}=\{0\} \Leftrightarrow r(B)=r(A B) \Leftrightarrow k_{5}=0, \\
& \mathcal{M}+\mathcal{N}=\mathbb{C}^{m} \Leftrightarrow \mathcal{M}^{\perp} \cap \mathcal{N}^{\perp}=\{0\} \Leftrightarrow r[A, B]=m \Leftrightarrow k_{6}=0 .
\end{aligned}
$$

(g) The following equivalent statements hold

$$
\begin{aligned}
& \mathcal{M} \subseteq \mathcal{N} \Leftrightarrow B A=A \Leftrightarrow \mathcal{M} \cap \mathcal{N}^{\perp}=\{0\} \text { and } A B=B A \Leftrightarrow k_{1}=k_{2}=k_{4}=0, \\
& \mathcal{M} \supseteq \mathcal{N} \Leftrightarrow A B=B \Leftrightarrow \mathcal{M}^{\perp} \cap \mathcal{N}=\{0\} \text { and } A B=B A \Leftrightarrow k_{1}=k_{2}=k_{5}=0, \\
& \mathcal{M}=\mathcal{N} \Leftrightarrow A=B \Leftrightarrow \mathcal{M} \cap \mathcal{N}^{\perp}=\mathcal{M}^{\perp} \cap \mathcal{N}=\{0\} \text { and } A B=B A \Leftrightarrow k_{1}=k_{2}=k_{4}=k_{5}=0, \\
& \mathcal{M} \perp \mathcal{N} \Leftrightarrow A B=0 \Leftrightarrow A B+B A=0 \Leftrightarrow A B A=0 \Leftrightarrow B A B=0 \Leftrightarrow A B A+B A B=0 \\
& \Leftrightarrow k_{1}=k_{2}=k_{3}=0, \\
& A-B \text { is nonsigular } \Leftrightarrow k_{3}=k_{6}=0, \\
& A B+B A \text { is nonsigular } \Leftrightarrow A B A+B A B \text { is nonsigular } \Leftrightarrow k_{4}=k_{5}=k_{6}=0, \\
& A B-B A \text { is nonsigular } \Leftrightarrow A B A-B A B \text { is nonsigular } \Leftrightarrow k_{3}=k_{4}=k_{5}=k_{6}=0, \\
& I_{m}-A B \text { is nonsigular } \Leftrightarrow I_{m}-A B A \text { is nonsigular } \Leftrightarrow I_{m}-B A B \text { is nonsigular } \Leftrightarrow k_{3}=0,
\end{aligned}
$$




$$
\begin{aligned}
& A-B A B \text { is nonsigular } \Leftrightarrow k_{3}=k_{5}=k_{6}=0, \\
& B-A B A \text { is nonsigular } \Leftrightarrow k_{3}=k_{4}=k_{6}=0 .
\end{aligned}
$$

Proof. It follows from direct verifications by (3.3)-(3.10) and the definitions of the Moore-Penrose inverses and group inverses of matrices.

It is easy to observe from Theorem 3.4(a) that

$$
\begin{array}{llrl}
\mathscr{R}(A B) & =\mathcal{M} \circ \mathcal{N}=\mathcal{M} \cap\left(\mathcal{M} \cap \mathcal{N}^{\perp}\right)^{\perp}, & & \mathscr{R}(A \widetilde{B})=\mathcal{M} \circ \mathcal{N}^{\perp}=\mathcal{M} \cap(\mathcal{M} \cap \mathcal{N})^{\perp}, \\
\mathscr{R}(\widetilde{A} B)=\mathcal{M}^{\perp} \circ \mathcal{N}=\mathcal{M}^{\perp} \cap\left(\mathcal{M}^{\perp} \cap \mathcal{N}^{\perp}\right)^{\perp}, & \mathscr{R}(\widetilde{A} \widetilde{B})=\mathcal{M}^{\perp} \circ \mathcal{N}^{\perp}=\mathcal{M}^{\perp} \cap\left(\mathcal{M}^{\perp} \cap \mathcal{N}\right)^{\perp}, \\
\mathscr{R}(B A)=\mathcal{N} \circ \mathcal{M}=\mathcal{N} \cap\left(\mathcal{M}^{\perp} \cap \mathcal{N}\right)^{\perp}, & & \mathscr{R}(\widetilde{B} A)=\mathcal{N}^{\perp} \circ \mathcal{M}=\mathcal{N}^{\perp} \cap\left(\mathcal{M}^{\perp} \cap \mathcal{N}^{\perp}\right)^{\perp}, \\
\mathscr{R}(B \widetilde{A})=\mathcal{N} \circ \mathcal{M}^{\perp}=\mathcal{N} \cap(\mathcal{M} \cap \mathcal{N})^{\perp}, & & \mathscr{R}(\widetilde{B} \widetilde{A})=\mathcal{N}^{\perp} \circ \mathcal{M}^{\perp}=\mathcal{N}^{\perp} \cap\left(\mathcal{M} \cap \mathcal{N}^{\perp}\right)^{\perp} .
\end{array}
$$

These identities can be used to establish many direct sum decomposition identities of $A, B, \widetilde{A}$, and $\widetilde{B}$ in (3.2) and their algebraic operations. In particular, we have the following consequences.

Corollary 3.5. Let $\mathcal{N}$ and $\mathcal{N}$ be two linear subspaces of $\mathbb{C}^{m}$, and assume that $A, B, \widetilde{A}$ and $\widetilde{B}$ in (3.2) are decomposed as (3.3)-(3.6). Then, the following results hold.

(a) The following intersection identities hold

$$
\begin{aligned}
& \mathscr{R}(A B \widetilde{A})=\mathscr{R}(A \widetilde{B} \widetilde{A})=\mathscr{R}(A B) \cap \mathscr{R}(A \widetilde{B})=\mathcal{M} \bullet \mathcal{N}, \\
& \mathscr{R}(B A \widetilde{B})=\mathscr{R}(B \widetilde{A} \widetilde{B})=\mathscr{R}(B A) \cap \mathscr{R}(B \widetilde{A})=\mathcal{N} \bullet \mathcal{M}, \\
& \mathscr{R}(\widetilde{A} B A)=\mathscr{R}(\widetilde{A} \widetilde{B} A)=\mathscr{R}(\widetilde{A} B) \cap \mathscr{R}(\widetilde{A} \widetilde{B})=\mathcal{M}^{\perp} \bullet \mathcal{N}, \\
& \mathscr{R}(\widetilde{B} A B)=\mathscr{R}(\widetilde{B} \widetilde{A} B)=\mathscr{R}(\widetilde{B} A) \cap \mathscr{R}(\widetilde{B} \widetilde{A})=\mathcal{N}^{\perp} \bullet \mathcal{M} .
\end{aligned}
$$

(b) The following direct sum decomposition identities hold

$$
\begin{aligned}
& \mathscr{R}(A B)=\mathscr{R}(A B A)=\mathscr{R}(A B \widetilde{A}) \oplus(\mathcal{M} \cap \mathcal{N})=(\mathcal{M} \bullet \mathcal{N}) \oplus(\mathcal{M} \cap \mathcal{N}), \\
& \mathscr{R}(A \widetilde{B})=\mathscr{R}(A \widetilde{B} A)=\mathscr{R}(A B \widetilde{A}) \oplus\left(\mathcal{M} \cap \mathcal{N}^{\perp}\right)=(\mathcal{N} \bullet \mathcal{N}) \oplus\left(\mathcal{M} \cap \mathcal{N}^{\perp}\right), \\
& \mathscr{R}(\widetilde{A} B)=\mathscr{R}(\widetilde{A} B \widetilde{A})=\mathscr{R}(\widetilde{A} B A) \oplus\left(\mathcal{M}^{\perp} \cap \mathcal{N}\right)=\left(\mathcal{M}^{\perp} \bullet \mathcal{N}\right) \oplus\left(\mathcal{M}^{\perp} \cap \mathcal{N}\right), \\
& \mathscr{R}(\widetilde{A} \widetilde{B})=\mathscr{R}(\widetilde{A} \widetilde{B} \widetilde{A})=\mathscr{R}(\widetilde{A} B A) \oplus\left(\mathcal{M}^{\perp} \cap \mathcal{N}^{\perp}\right)=\left(\mathcal{M}^{\perp} \bullet \mathcal{N}\right) \oplus\left(\mathcal{M}^{\perp} \cap \mathcal{N}^{\perp}\right), \\
& \mathscr{R}(B A)=\mathscr{R}(B A B)=\mathscr{R}(B A \widetilde{B}) \oplus(\mathcal{M} \cap \mathcal{N})=(\mathcal{N} \bullet \mathcal{M}) \oplus(\mathcal{M} \cap \mathcal{N}), \\
& \mathscr{R}(B \widetilde{A})=\mathscr{R}(B \widetilde{A} B)=\mathscr{R}(B A \widetilde{B}) \oplus\left(\mathcal{M}^{\perp} \cap \mathcal{N}\right)=(\mathcal{N} \bullet \mathcal{N}) \oplus\left(\mathcal{M}^{\perp} \cap \mathcal{N}\right), \\
& \mathscr{R}(\widetilde{B} A)=\mathscr{R}(\widetilde{B} A \widetilde{B})=\mathscr{R}(\widetilde{B} A B) \oplus\left(\mathcal{M} \cap \mathcal{N}^{\perp}\right)=\left(\mathcal{N}^{\perp} \bullet \mathcal{M}\right) \oplus\left(\mathcal{M} \cap \mathcal{N}^{\perp}\right), \\
& \mathscr{R}(\widetilde{B} \widetilde{A})=\mathscr{R}(\widetilde{B} \widetilde{A} \widetilde{B})=\mathscr{R}(\widetilde{B} A B) \oplus\left(\mathcal{M}^{\perp} \cap \mathcal{N}^{\perp}\right)=\left(\mathcal{N}^{\perp} \bullet \mathcal{M}\right) \oplus\left(\mathcal{M}^{\perp} \cap \mathcal{N}^{\perp}\right) .
\end{aligned}
$$

(c) The ranges of the products of $A, B, \widetilde{A}$ and $\widetilde{B}$ can be decomposed as

$$
\begin{aligned}
& \mathscr{R}(A B+B A)=\mathscr{R}(A B A+B A B)=\mathscr{R}(A B)+\mathscr{R}(B A)=(\mathcal{M} \circ \mathcal{N})+(\mathcal{N} \circ \mathcal{N}), \\
& \mathscr{R}(A \widetilde{B}+\widetilde{B} A)=\mathscr{R}(A \widetilde{B} A+\widetilde{B} A \widetilde{B})=\mathscr{R}(A \widetilde{B})+\mathscr{R}(\widetilde{B} A)=\left(\mathcal{M} \circ \mathcal{N}^{\perp}\right)+\left(\mathcal{N}^{\perp} \circ \mathcal{M}\right), \\
& \mathscr{R}(\widetilde{A} B+B \widetilde{A})=\mathscr{R}(\widetilde{A} B \widetilde{A}+B \widetilde{A} B)=\mathscr{R}(\widetilde{A} B)+\mathscr{R}(B \widetilde{A})=\left(\mathcal{M}^{\perp} \circ \mathcal{N}\right)+\left(\mathcal{N} \circ \mathcal{M}^{\perp}\right), \\
& \mathscr{R}(\widetilde{A} \widetilde{B} \widetilde{B} \widetilde{A})=\mathscr{R}(\widetilde{A} \widetilde{B} \widetilde{A} \widetilde{B} \widetilde{B})=\mathscr{R}(\widetilde{A} \widetilde{B})+\mathscr{R}(\widetilde{B} \widetilde{A})=\left(\mathcal{M}^{\perp} \circ \mathcal{N}^{\perp}\right)+\left(\mathcal{N}^{\perp} \circ \mathcal{M}^{\perp}\right), \\
& \mathscr{R}(A B+\widetilde{B} \widetilde{A})=\mathscr{R}(A B A+\widetilde{B} \widetilde{A} \widetilde{B})=\mathscr{R}(A B) \oplus \mathscr{R}(\widetilde{B} \widetilde{A})=(\mathcal{M} \circ \mathcal{N}) \oplus\left(\mathcal{N}^{\perp} \circ \mathcal{M}^{\perp}\right), \\
& \mathscr{R}(A \widetilde{B}+B \widetilde{A})=\mathscr{R}(A \widetilde{B} A+B \widetilde{A} B)=\mathscr{R}(A \widetilde{B}) \oplus \mathscr{R}(B \widetilde{A})=\left(\mathcal{M} \circ \mathcal{N}^{\perp}\right) \oplus\left(\mathcal{N} \circ \mathcal{M}^{\perp}\right), \\
& \mathscr{R}(\widetilde{A} B+\widetilde{B} A)=\mathscr{R}(\widetilde{A} B \widetilde{A}+\widetilde{B} A \widetilde{B})=\mathscr{R}(\widetilde{A} B) \oplus \mathscr{R}(\widetilde{B} A)=\left(\mathcal{M}^{\perp} \circ \mathcal{N}\right) \oplus\left(\mathcal{N}^{\perp} \circ \mathcal{M}\right), \\
& \mathscr{R}(\widetilde{A} \widetilde{B}+B A)=\mathscr{R}(\widetilde{A} \widetilde{B} \widetilde{A}+B A B)=\mathscr{R}(\widetilde{A} \widetilde{B}) \oplus \mathscr{R}(B A)=\left(\mathcal{M}^{\perp} \circ \mathcal{N} \mathcal{N}^{\perp}\right) \oplus(\mathcal{N} \circ \mathcal{M}),
\end{aligned}
$$

and

$$
\mathscr{R}(A B \widetilde{A}+\widetilde{A} B A)=\mathscr{R}(A B \widetilde{A}) \oplus \mathscr{R}(\widetilde{A} B A)=(\mathcal{M} \bullet \mathcal{N}) \oplus\left(\mathcal{M}^{\perp} \bullet \mathcal{N}\right),
$$




$$
\begin{aligned}
& \mathscr{R}(B A \widetilde{B}+\widetilde{B} A B)=\mathscr{R}(B A \widetilde{B}) \oplus \mathscr{R}(\widetilde{B} A B)=(\mathcal{N} \bullet \mathcal{N}) \oplus\left(\mathcal{N}^{\perp} \bullet \mathcal{M}\right), \\
& \mathscr{R}(A B \widetilde{A}+B A \widetilde{B})=\mathscr{R}(A B \widetilde{A}) \oplus \mathscr{R}(B A \widetilde{B})=(\mathcal{M} \bullet \mathcal{N}) \oplus(\mathcal{N} \bullet \mathcal{M}), \\
& \mathscr{R}(\widetilde{A} B A+\widetilde{B} A B)=\mathscr{R}(\widetilde{A} B A) \oplus \mathscr{R}(\widetilde{B} A B)=\left(\mathcal{M}^{\perp} \bullet \mathcal{N}\right) \oplus\left(\mathcal{N}^{\perp} \bullet \mathcal{M}\right) .
\end{aligned}
$$

(d) The following orthogonal direct sum decomposition identities on the ranges of $A B, B A$ and their operations hold

$$
\begin{aligned}
& \mathscr{R}(A B-B A)=\mathscr{R}(A \widetilde{B}-\widetilde{B} A)=\mathscr{R}(A \widetilde{B}-\widetilde{B} A)=\mathscr{R}(\widetilde{A} \widetilde{B}-\widetilde{B} \widetilde{A}) \\
& =\mathscr{R}(A B A-B A B)=\mathscr{R}(A \widetilde{B} A-\widetilde{B} A \widetilde{B}) \\
& =\mathscr{R}(\widetilde{A} B \widetilde{A}-B \widetilde{A} B)=\mathscr{R}(\widetilde{A} \widetilde{B} \widetilde{A}-\widetilde{B} \widetilde{A} \widetilde{B}) \\
& =(\mathcal{N} \cdot \mathcal{N}) \oplus(\mathcal{N} \cdot \mathcal{M})=\left(\mathcal{M}^{\perp} \bullet \mathcal{N}\right) \oplus\left(\mathcal{N}^{\perp} \cdot \mathcal{M}\right), \\
& \mathscr{R}(A B+B A)=\mathscr{R}(A B-B A) \oplus(\mathcal{M} \cap \mathcal{N})=\mathcal{M} \bullet \mathcal{N} \oplus \mathcal{N} \bullet \mathcal{M} \oplus(\mathcal{M} \cap \mathcal{N}) \oplus(\mathcal{M} \cap \mathcal{N}) \\
& =\left(\mathcal{M}^{\perp} \cdot \mathcal{N}\right) \oplus\left(\mathcal{N}^{\perp} \cdot \mathcal{M}\right) \oplus(\mathcal{M} \cap \mathcal{N}), \\
& \mathscr{R}(A-B)=\mathscr{R}(A B-B A) \oplus\left(\mathcal{M} \cap \mathcal{N}^{\perp}\right) \oplus\left(\mathcal{M}^{\perp} \cap \mathcal{N}\right) \\
& =(\mathcal{M} \bullet \mathcal{N}) \oplus(\mathcal{N} \bullet \mathcal{M}) \oplus\left(\mathcal{M} \cap \mathcal{N}^{\perp}\right) \oplus\left(\mathcal{M}^{\perp} \cap \mathcal{N}\right) \oplus\left(\mathcal{M} \cap \mathcal{N}^{\perp}\right) \\
& =\left(\mathcal{M}^{\perp} \bullet \mathcal{N}\right) \oplus\left(\mathcal{N}^{\perp} \bullet \mathcal{M}\right) \oplus\left(\mathcal{M} \cap \mathcal{N}^{\perp}\right) \oplus\left(\mathcal{M}^{\perp} \cap \mathcal{N}\right) \oplus\left(\mathcal{M} \cap \mathcal{N}^{\perp}\right), \\
& \mathscr{R}\left(I_{m}-A-B\right)=\mathscr{R}(A B+B A) \oplus\left(\mathcal{M}^{\perp} \cap \mathcal{N}^{\perp}\right)=\mathscr{R}(A B-B A) \oplus(\mathcal{M} \cap \mathcal{N}) \oplus\left(\mathcal{M}^{\perp} \cap \mathcal{N}^{\perp}\right) \\
& =(\mathcal{K} \cdot \mathcal{N}) \oplus(\mathcal{N} \cdot \mathcal{K}) \oplus(\mathcal{K} \cap \mathcal{N}) \oplus\left(\mathcal{K}^{\perp} \cap \mathcal{N}^{\perp}\right) \\
& =\left(\mathcal{M}^{\perp} \cdot \mathcal{N}\right) \oplus\left(\mathcal{N}^{\perp} \cdot \mathcal{N}\right) \oplus(\mathcal{K} \cap \mathcal{N}) \oplus\left(\mathcal{M}^{\perp} \cap \mathcal{N}\right), \\
& \mathscr{R}\left(I_{m}-A B\right)=\mathscr{R}\left(I_{m}-B A\right)=\mathscr{R}\left(I_{m}-A B A\right)=\mathscr{R}\left(I_{m}-B A B\right) \\
& =\mathscr{R}(A B-B A) \oplus\left(\mathcal{M} \cap \mathcal{N}^{\perp}\right) \oplus\left(\mathcal{M}^{\perp} \cap \mathcal{N}\right) \oplus\left(\mathcal{M}^{\perp} \cap \mathcal{N}^{\perp}\right) \\
& =(\mathcal{M} \bullet \mathcal{N}) \oplus(\mathcal{N} \cdot \mathcal{M}) \oplus\left(\mathcal{M} \cap \mathcal{N}^{\perp}\right) \oplus\left(\mathcal{M}^{\perp} \cap \mathcal{N}\right) \oplus\left(\mathcal{M}^{\perp} \cap \mathcal{N}^{\perp}\right) \\
& =\left(\mathcal{M}^{\perp} \bullet \mathcal{N}\right) \oplus\left(\mathcal{N}^{\perp} \bullet \mathcal{M}\right) \oplus\left(\mathcal{M} \cap \mathcal{N}^{\perp}\right) \oplus\left(\mathcal{M}^{\perp} \cap \mathcal{N}\right) \oplus\left(\mathcal{M}^{\perp} \cap \mathcal{N}^{\perp}\right), \\
& \mathscr{R}(A-B A B)=\mathscr{R}(A B-B A) \oplus\left(\mathcal{M} \cap \mathcal{N}^{\perp}\right)=(\mathcal{M} \bullet \mathcal{N}) \oplus(\mathcal{N} \bullet \mathcal{M}) \oplus\left(\mathcal{M} \cap \mathcal{N}^{\perp}\right) \\
& =\left(\mathcal{M}^{\perp} \bullet \mathcal{N}\right) \oplus\left(\mathcal{N}^{\perp} \bullet \mathcal{M}\right) \oplus\left(\mathcal{M} \cap \mathcal{N}^{\perp}\right), \\
& \mathscr{R}(B-A B A)=\mathscr{R}(A B-B A) \oplus\left(\mathcal{M}^{\perp} \cap \mathcal{N}\right)=(\mathcal{M} \bullet \mathcal{N}) \oplus(\mathcal{N} \bullet \mathcal{M}) \oplus\left(\mathcal{M}^{\perp} \cap \mathcal{N}\right) \\
& =\left(\mathcal{M}^{\perp} \bullet \mathcal{N}\right) \oplus\left(\mathcal{N}^{\perp} \bullet \mathcal{M}\right) \oplus\left(\mathcal{M}^{\perp} \cap \mathcal{N}\right) .
\end{aligned}
$$

(e) The following orthogonal direct sum decomposition identities on $\mathcal{N}, \mathcal{N}$, and their operations hold

$$
\begin{aligned}
& \mathcal{M}=\mathscr{R}(A B) \oplus\left(\mathcal{M} \cap \mathcal{N}^{\perp}\right)=\mathscr{R}(A \widetilde{B}) \oplus(\mathcal{M} \cap \mathcal{N})=\mathscr{R}(A B \widetilde{A}) \oplus(\mathcal{M} \cap \mathcal{N}) \oplus\left(\mathcal{M} \cap \mathcal{N}^{\perp}\right), \\
& \mathcal{N}=\mathscr{R}(B A) \oplus\left(\mathcal{M}^{\perp} \cap \mathcal{N}\right)=\mathscr{R}(B \widetilde{A}) \oplus(\mathcal{M} \cap \mathcal{N})=\mathscr{R}(B A \widetilde{B}) \oplus(\mathcal{M} \cap \mathcal{N}) \oplus\left(\mathcal{M}^{\perp} \cap \mathcal{N}\right), \\
& \mathcal{M}^{\perp}=\mathscr{R}(\widetilde{A} B) \oplus\left(\mathcal{M}^{\perp} \cap \mathcal{N}^{\perp}\right)=\mathscr{R}(\widetilde{A} \widetilde{B}) \oplus\left(\mathcal{M}^{\perp} \cap \mathcal{N}\right)=\mathscr{R}(\widetilde{A} B A) \oplus\left(\mathcal{M}^{\perp} \cap \mathcal{N}\right) \oplus\left(\mathcal{M}^{\perp} \cap \mathcal{N}^{\perp}\right) \text {, } \\
& \mathcal{N}^{\perp}=\mathscr{R}(\widetilde{B} A) \oplus\left(\mathcal{M}^{\perp} \cap \mathcal{N}^{\perp}\right)=\mathscr{R}(\widetilde{B} \widetilde{A}) \oplus\left(\mathcal{M} \cap \mathcal{N}^{\perp}\right)=\mathscr{R}(\widetilde{B} A B) \oplus\left(\mathcal{M} \cap \mathcal{N}^{\perp}\right) \oplus\left(\mathcal{M}^{\perp} \cap \mathcal{N}^{\perp}\right), \\
& \mathcal{M}+\mathcal{N}=\mathscr{R}(A-B) \oplus(\mathcal{M} \cap \mathcal{N})=\mathscr{R}(A B+B A) \oplus\left(\mathcal{M} \cap \mathcal{N}^{\perp}\right) \oplus\left(\mathcal{M}^{\perp} \cap \mathcal{N}\right) \\
& =\mathscr{R}(A B-B A) \oplus(\mathcal{M} \cap \mathcal{N}) \oplus\left(\mathcal{M} \cap \mathcal{N}^{\perp}\right) \oplus\left(\mathcal{M}^{\perp} \cap \mathcal{N}\right) \\
& =\mathscr{R}(A B \tilde{A}) \oplus \mathscr{R}(\tilde{A} B A) \oplus(\mathcal{M} \cap \mathcal{N}) \oplus\left(\mathcal{M} \cap \mathcal{N}^{\perp}\right) \oplus\left(\mathcal{M}^{\perp} \cap \mathcal{N}\right) \\
& =\mathscr{R}(B A \widetilde{B}) \oplus \mathscr{R}(\widetilde{B} A B) \oplus(\mathcal{M} \cap \mathcal{N}) \oplus\left(\mathcal{M} \cap \mathcal{N}^{\perp}\right) \oplus\left(\mathcal{M}^{\perp} \cap \mathcal{N}\right) \\
& =\mathscr{R}(A B \tilde{A}) \oplus \mathscr{R}(B A \widetilde{B}) \oplus(\mathcal{M} \cap \mathcal{N}) \oplus\left(\mathcal{M} \cap \mathcal{N}^{\perp}\right) \oplus\left(\mathcal{M}^{\perp} \cap \mathcal{N}\right) \\
& =\mathscr{R}(\widetilde{A} B A) \oplus \mathscr{R}(\widetilde{B} A B) \oplus(\mathcal{M} \cap \mathcal{N}) \oplus\left(\mathcal{M} \cap \mathcal{N}^{\perp}\right) \oplus\left(\mathcal{M}^{\perp} \cap \mathcal{N}\right) \text {, } \\
& \mathcal{M}^{\perp}+\mathcal{N}^{\perp}=\mathscr{R}(\widetilde{A} B+\widetilde{B} A) \oplus\left(\mathcal{M}^{\perp} \cap \mathcal{N}^{\perp}\right)=[\mathscr{R}(\widetilde{A} B)+\mathscr{R}(\widetilde{B} A)] \oplus\left(\mathcal{M}^{\perp} \cap \mathcal{N}^{\perp}\right) \\
& =\mathscr{R}(A-B) \oplus\left(\mathcal{M}^{\perp} \cap \mathcal{N}^{\perp}\right)=\mathscr{R}(\widetilde{A} \widetilde{B}+\widetilde{B} \widetilde{A}) \oplus\left(\mathcal{M} \cap \mathcal{N}^{\perp}\right) \oplus\left(\mathcal{M}^{\perp} \cap \mathcal{N}\right)
\end{aligned}
$$




$$
\begin{aligned}
& =\mathscr{R}(\widetilde{A} \widetilde{B}-\widetilde{B} \widetilde{A}) \oplus\left(\mathcal{M} \cap \mathcal{N}^{\perp}\right) \oplus\left(\mathcal{M}^{\perp} \cap \mathcal{N}\right) \oplus\left(\mathcal{M}^{\perp} \cap \mathcal{N}^{\perp}\right) \\
& =\mathscr{R}(A B \widetilde{A}) \oplus \mathscr{R}(\tilde{A} B A) \oplus\left(\mathcal{M} \cap \mathcal{N}^{\perp}\right) \oplus\left(\mathcal{M}^{\perp} \cap \mathcal{N}\right) \oplus\left(\mathcal{M}^{\perp} \cap \mathcal{N}^{\perp}\right) \\
& =\mathscr{R}(B A \widetilde{B}) \oplus \mathscr{R}(\widetilde{B} A B) \oplus\left(\mathcal{M} \cap \mathcal{N}^{\perp}\right) \oplus\left(\mathcal{M}^{\perp} \cap \mathcal{N}\right) \oplus\left(\mathcal{M}^{\perp} \cap \mathcal{N}^{\perp}\right) \\
& =\mathscr{R}(A B \widetilde{A}) \oplus \mathscr{R}(B A \widetilde{B}) \oplus\left(\mathcal{M} \cap \mathcal{N}^{\perp}\right) \oplus\left(\mathcal{M}^{\perp} \cap \mathcal{N}\right) \oplus\left(\mathcal{M}^{\perp} \cap \mathcal{N}^{\perp}\right) \\
& =\mathscr{R}(\widetilde{A} B A) \oplus \mathscr{R}(\widetilde{B} A B) \oplus\left(\mathcal{M} \cap \mathcal{N}^{\perp}\right) \oplus\left(\mathcal{M}^{\perp} \cap \mathcal{N}\right) \oplus\left(\mathcal{M}^{\perp} \cap \mathcal{N}^{\perp}\right) \text {, } \\
& \mathbb{C}^{m}=\mathscr{R}(A-B) \oplus(\mathcal{M} \cap \mathcal{N}) \oplus\left(\mathcal{M}^{\perp} \cap \mathcal{N}^{\perp}\right) \\
& =\mathscr{R}(A B+B A) \oplus\left(\mathcal{M} \cap \mathcal{N}^{\perp}\right) \oplus\left(\mathcal{M}^{\perp} \cap \mathcal{N}\right) \oplus\left(\mathcal{M}^{\perp} \cap \mathcal{N}^{\perp}\right) \\
& =\mathscr{R}(\widetilde{A} \widetilde{B}+\widetilde{B} \widetilde{A}) \oplus(\mathcal{M} \cap \mathcal{N}) \oplus\left(\mathcal{M} \cap \mathcal{N}^{\perp}\right) \oplus\left(\mathcal{M}^{\perp} \cap \mathcal{N}\right) \\
& =\mathscr{R}(A B-B A) \oplus(\mathcal{M} \cap \mathcal{N}) \oplus\left(\mathcal{M} \cap \mathcal{N}^{\perp}\right) \oplus\left(\mathcal{M}^{\perp} \cap \mathcal{N}\right) \oplus\left(\mathcal{M}^{\perp} \cap \mathcal{N}^{\perp}\right) \\
& =\mathscr{R}(A B \widetilde{A}) \oplus \mathscr{R}(\widetilde{A} B A) \oplus(\mathcal{M} \cap \mathcal{N}) \oplus\left(\mathcal{M} \cap \mathcal{N}^{\perp}\right) \oplus\left(\mathcal{M}^{\perp} \cap \mathcal{N}\right) \oplus\left(\mathcal{M}^{\perp} \cap \mathcal{N}^{\perp}\right) \\
& =\mathscr{R}(B A \widetilde{B}) \oplus \mathscr{R}(\widetilde{B} A B) \oplus(\mathcal{M} \cap \mathcal{N}) \oplus\left(\mathcal{M} \cap \mathcal{N}^{\perp}\right) \oplus\left(\mathcal{M}^{\perp} \cap \mathcal{N}\right) \oplus\left(\mathcal{M}^{\perp} \cap \mathcal{N}^{\perp}\right) \\
& =\mathscr{R}(A B \widetilde{A}) \oplus \mathscr{R}(B A \widetilde{B}) \oplus(\mathcal{M} \cap \mathcal{N}) \oplus\left(\mathcal{M} \cap \mathcal{N}^{\perp}\right) \oplus\left(\mathcal{M}^{\perp} \cap \mathcal{N}\right) \oplus\left(\mathcal{M}^{\perp} \cap \mathcal{N}^{\perp}\right) \\
& =\mathscr{R}(\widetilde{A} B A) \oplus \mathscr{R}(\widetilde{B} A B) \oplus(\mathcal{M} \cap \mathcal{N}) \oplus\left(\mathcal{M} \cap \mathcal{N}^{\perp}\right) \oplus\left(\mathcal{M}^{\perp} \cap \mathcal{N}\right) \oplus\left(\mathcal{M}^{\perp} \cap \mathcal{N}^{\perp}\right) \text {, }
\end{aligned}
$$

and the following decomposition identities of orthogonal projectors hold

$$
\begin{aligned}
& A=P_{\mathscr{R}(A B)}+P_{\mathcal{M} \cap \mathcal{N}^{\perp}}=P_{\mathscr{R}(A \widetilde{B})}+P_{\mathcal{M} \cap \mathcal{N}}=P_{\mathscr{R}(A B \widetilde{A})}+P_{\mathcal{M N \cap \mathcal { N }}}+P_{\mathcal{M} \cap \mathcal{N}^{\perp}}, \\
& B=P_{\mathscr{R}(B A)}+P_{\mathcal{M} \perp \cap \mathcal{N}}=P_{\mathscr{R}(B \widetilde{A})}+P_{\mathcal{M} \cap \mathcal{N}}=P_{\mathscr{R}(B A \widetilde{B})}+P_{\mathcal{M} \cap \mathcal{N}}+P_{\mathcal{M} \perp \cap \mathcal{N}}, \\
& A+B=P_{\mathscr{R}(A B)}+P_{\mathscr{R}(B A)}+P_{\mathcal{M} \cap \mathcal{N} \perp}+P_{\mathcal{M} \perp \cap \mathcal{N}}=P_{\mathscr{R}(A \widetilde{B})}+P_{\mathscr{R}(B \widetilde{A})}+2 P_{\mathcal{M} \cap \mathcal{N}} \\
& =P_{\mathscr{R}(A B \tilde{A})}+P_{\mathscr{R}(B A \widetilde{B})}+2 P_{\mathcal{M} \cap \mathcal{N}}+P_{\mathcal{M} \cap \mathcal{N}^{\perp}}+P_{\mathcal{M}^{\perp} \cap \mathcal{N}}, \\
& P_{\mathcal{M}+\mathcal{N}}=P_{\mathscr{R}(A B+B A)}+P_{\mathcal{M} \cap \mathcal{N}^{\perp}}+P_{\mathcal{M} \perp \cap \mathcal{N}}=P_{\mathscr{R}(A B-B A)}+P_{\mathcal{M} \cap \mathcal{N}}+P_{\mathcal{M} \cap \mathcal{N}^{\perp}}+P_{\mathcal{M} \perp \perp \mathcal{N}} \\
& =P_{\mathscr{R}(A B \widetilde{A})}+P_{\mathscr{R}(\widetilde{A} B A)}+P_{\mathcal{M} \cap \mathcal{N}}+P_{\mathcal{M} \cap \mathcal{N}^{\perp}}+P_{\mathcal{\mathcal { M } ^ { \perp }} \cap \mathcal{N}} \\
& =P_{\mathscr{R}(B A \widetilde{B})}+P_{\mathscr{R}(\widetilde{B} A B)}+P_{\mathcal{M} \cap \mathcal{N}}+P_{\mathcal{M} \cap \mathcal{N}^{\perp}}+P_{\mathcal{M}^{\perp} \cap \mathcal{N}} \text {, } \\
& \widetilde{A}=P_{\mathscr{R}(\widetilde{A} B)}+P_{\mathcal{M}^{\perp} \cap \mathcal{N}^{\perp}}=P_{\mathscr{R}(\tilde{A} \widetilde{B})}+P_{\mathcal{M}^{\perp} \cap \mathcal{N}}=P_{\mathscr{R}(\tilde{A} B A)}+P_{\mathcal{M}^{\perp} \cap \mathcal{N}}+P_{\mathcal{M}^{\perp} \cap \mathcal{N}^{\perp}} \text {, } \\
& \widetilde{B}=P_{\mathscr{R}(\widetilde{B} A)}+P_{\mathcal{M}^{\perp} \cap \mathcal{N}^{\perp}}=P_{\mathscr{R}(\widetilde{B} \widetilde{A})}+P_{\mathcal{M} \cap \mathcal{N}^{\perp}}=P_{\mathscr{R}(\widetilde{B} A B)}+P_{\mathcal{M} \cap \mathcal{N}^{\perp}}+P_{\mathcal{M}^{\perp} \cap \mathcal{N}^{\perp}} \text {, } \\
& \widetilde{A}+\widetilde{B}=P_{\mathscr{R}(\widetilde{A} B)}+P_{\mathscr{R}(\widetilde{B} A)}+2 P_{\mathcal{M}^{\perp} \cap \mathcal{N}^{\perp}}=P_{\mathscr{R}(\widetilde{A} \widetilde{B})}+P_{\mathscr{R}(\widetilde{B} \widetilde{A})}+P_{\mathcal{M}^{\perp} \cap \mathcal{N}}+P_{\mathcal{M} \cap \mathcal{N}^{\perp}} \\
& =P_{\mathscr{R}(\widetilde{A} B A)}+P_{\mathscr{R}(\widetilde{B} A B)}+P_{\mathcal{M}^{\perp} \cap \mathcal{N}}+P_{\mathcal{M} \cap \mathcal{N}^{\perp}}+2 P_{\mathcal{M}^{\perp} \cap \mathcal{N}^{\perp}} \text {, } \\
& P_{\mathcal{M}^{\perp}+\mathcal{N}^{\perp}}=P_{\mathscr{R}(\widetilde{A} \widetilde{B}+\widetilde{B} \widetilde{A})}+P_{\mathcal{M} \cap \mathcal{N}^{\perp}}+P_{\mathcal{M}^{\perp} \cap \mathcal{N}}=P_{\mathscr{R}(\widetilde{A} \widetilde{B}-\widetilde{B} \widetilde{A})}+P_{\mathcal{M} \cap \mathcal{N}^{\perp}}+P_{\mathcal{M}^{\perp} \cap \mathcal{N}}+P_{\mathcal{M}^{\perp} \cap \mathcal{N} \perp}
\end{aligned}
$$

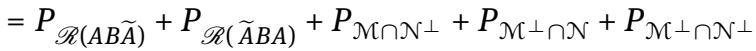

$$
\begin{aligned}
& =P_{\mathscr{R}(B A \widetilde{B})}+P_{\mathscr{R}(\widetilde{B} A B)}+P_{\mathcal{M} \cap \mathcal{N}^{\perp}}+P_{\mathcal{M} \perp \cap \mathcal{N}}+P_{\mathcal{M} \perp \cap \mathcal{N}^{\perp}} \text {, } \\
& I_{m}=P_{\mathscr{R}(A-B)}+P_{\mathcal{M} \cap \mathcal{N}}+P_{\mathcal{M}^{\perp} \cap \mathcal{N}^{\perp}}=P_{\mathscr{R}(A B+B A)}+P_{\mathcal{M} \cap \mathcal{N}^{\perp}}+P_{\mathcal{M}^{\perp} \cap \mathcal{N}}+P_{\mathcal{M} \perp \cap \mathcal{N} \perp} \\
& =P_{\mathscr{R}(\widetilde{A} \widetilde{B}+\widetilde{B} \widetilde{A})}+P_{\mathcal{M} \cap \mathcal{N}}+P_{\mathcal{M} \cap \mathcal{N}^{\perp}}+P_{\mathcal{M}}^{\perp \cap \mathcal{N}} \\
& =P_{\mathscr{R}(A B-B A)}+P_{\mathcal{M} \cap \mathcal{N}}+P_{\mathcal{M} \cap \mathcal{N}^{\perp}}+P_{\mathcal{M}^{\perp} \cap \mathcal{N}}+P_{\mathcal{M} \perp \cap \mathcal{N}^{\perp}}
\end{aligned}
$$

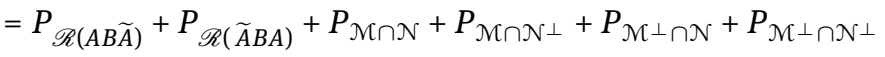

$$
\begin{aligned}
& =P_{\mathscr{R}(B A \widetilde{B})}+P_{\mathscr{R}(\widetilde{B} A B)}+P_{\mathcal{M} \cap \mathcal{N}}+P_{\mathcal{M} \cap \mathcal{N}^{\perp}}+P_{\mathcal{M}^{\perp} \cap \mathcal{N}}+P_{\mathcal{M}^{\perp} \cap \mathcal{N}^{\perp}} \\
& =P_{\mathscr{R}(A B \tilde{A})}+P_{\mathscr{R}(B A \widetilde{B})}+P_{\mathcal{M} \cap \mathcal{N}}+P_{\mathcal{M} \cap \mathcal{N}^{\perp}}+P_{\mathcal{M}}{ }^{\perp \cap \mathcal{N}}+P_{\mathcal{M}{ }^{\perp} \cap \mathcal{N}^{\perp}} \\
& =P_{\mathscr{R}(\widetilde{A} B A)}+P_{\mathscr{R}(\widetilde{B} A B)}+P_{\mathcal{M} \cap \mathcal{N}}+P_{\mathcal{M} \cap \mathcal{N}^{\perp}}+P_{\mathcal{M}^{\perp} \cap \mathcal{N}}+P_{\mathcal{M}^{\perp} \cap \mathcal{N}^{\perp}} \text {. }
\end{aligned}
$$

(f) The following set identities hold

$$
\mathscr{N}(A+B)=\mathcal{M}^{\perp} \cap \mathcal{N}^{\perp},
$$




$$
\begin{aligned}
\mathscr{N}(A-B) & =(\mathcal{H} \cap \mathcal{N}) \oplus\left(\mathcal{M}^{\perp} \cap \mathcal{N}^{\perp}\right), \\
\mathscr{N}\left(I_{m}-A-B\right) & =\left(\mathcal{M} \cap \mathcal{N}^{\perp}\right) \oplus\left(\mathcal{M}^{\perp} \cap \mathcal{N}\right), \\
\mathscr{N}(A B+B A) & =\mathscr{N}(A B A+B A B)=\left(\mathcal{M} \cap \mathcal{N}^{\perp}\right) \oplus\left(\mathcal{M}^{\perp} \cap \mathcal{N}\right) \oplus\left(\mathcal{K}^{\perp} \cap \mathcal{N}^{\perp}\right), \\
\mathscr{N}(A B-B A) & =\mathscr{N}(A B A-B A B)=\mathscr{N}(A B \widetilde{A}+\widetilde{A} B A)=\mathscr{N}(B A \widetilde{B}+\widetilde{B} A B) \\
& =\mathscr{N}(\widetilde{A} B A) \cap \mathscr{N}(A B \widetilde{A})=\mathscr{N}(\widetilde{B} A B) \cap \mathscr{N}(B A \widetilde{B}) \\
& =(\mathcal{M} \cap \mathcal{N}) \oplus\left(\mathcal{M} \cap \mathcal{N}^{\perp}\right) \oplus\left(\mathcal{M}^{\perp} \cap \mathcal{N}\right) \oplus\left(\mathcal{M}^{\perp} \cap \mathcal{N}^{\perp}\right), \\
\mathscr{N}\left(I_{m}-A B\right) & =\mathscr{N}\left(I_{m}-B A\right)=\mathscr{N}\left(I_{m}-A B A\right)=\mathscr{N}\left(I_{m}-B A B\right)=\mathcal{M} \cap \mathcal{N}, \\
\mathscr{N}(A-B A B) & =(\mathcal{M} \cap \mathcal{N}) \oplus\left(\mathcal{M}^{\perp} \cap \mathcal{N}\right) \oplus\left(\mathcal{M}^{\perp} \cap \mathcal{N}^{\perp}\right), \\
\mathscr{N}(B-A B A) & =(\mathcal{M} \cap \mathcal{N}) \oplus\left(\mathcal{M} \cap \mathcal{N} \mathcal{N}^{\perp}\right) \oplus\left(\mathcal{M}^{\perp} \cap \mathcal{N}^{\perp}\right) .
\end{aligned}
$$

Based on the given two linear subspaces $\mathcal{M}$ and $\mathcal{N}$, we define eight new linear subspaces from the ranges of the products of $A, B, \widetilde{A}$ and $\widetilde{B}$ in (3.2) as follows

$$
\begin{array}{ll}
\mathcal{S}_{1}=\mathscr{R}(A B)=\mathscr{R}(A B A), & \mathcal{T}_{1}=\mathscr{R}(B A)=\mathscr{R}(B A B), \\
\mathcal{S}_{2}=\mathscr{R}(A \widetilde{B})=\mathscr{R}(A \widetilde{B} A), & \mathcal{T}_{2}=\mathscr{R}(\widetilde{B} A)=\mathscr{R}(\widetilde{B} A \widetilde{B}), \\
\mathcal{S}_{3}=\mathscr{R}(\widetilde{A} B)=\mathscr{R}(\widetilde{A} B \widetilde{A}), & \mathcal{T}_{3}=\mathscr{R}(B \widetilde{A})=\mathscr{R}(B \widetilde{A} B), \\
\mathcal{S}_{4}=\mathscr{R}(\widetilde{A} \widetilde{B})=\mathscr{R}(\widetilde{A} \widetilde{B} \widetilde{A}), & \mathcal{T}_{4}=\mathscr{R}(\widetilde{B} \widetilde{A})=\mathscr{R}(\widetilde{B} \widetilde{A} \widetilde{B}) .
\end{array}
$$

Accordingly, the eight orthogonal projectors on the eight linear subspaces can be represented as

$$
\begin{array}{ll}
A_{1}=P_{\mathcal{S}_{1}}=(A B)(A B)^{\dagger}, & B_{1}=P_{\mathcal{T}_{1}}=(B A)(B A)^{\dagger}, \\
A_{2}=P_{\mathcal{S}_{2}}=(A \widetilde{B})(A \widetilde{B})^{\dagger}, & B_{2}=P_{\mathcal{T}_{2}}=(\widetilde{B} A)(\widetilde{B} A)^{\dagger}, \\
A_{3}=P_{\mathcal{S}_{3}}=(\widetilde{A} B)(\widetilde{A} B)^{\dagger}, & B_{3}=P_{\mathcal{T}_{3}}=(B \widetilde{A})(B \widetilde{A})^{\dagger}, \\
A_{4}=P_{\mathcal{S}_{4}}=(\widetilde{A} \widetilde{B})(\widetilde{A} \widetilde{B})^{\dagger}, & B_{4}=P_{\mathcal{T}_{4}}=(\widetilde{B} \widetilde{A})(\widetilde{B} \widetilde{A})^{\dagger} .
\end{array}
$$

Concerning the algebraic properties of these linear subspaces, we have the following results.

Corollary 3.6. Let $\mathcal{M}$ and $\mathcal{N}$ be two linear subspaces of $\mathbb{C}^{m}, A, B, \widetilde{A}$ and $\widetilde{B}$ in (3.2), $\mathcal{S}_{i}, \mathcal{T}_{i}, A_{i}$ and $B_{i}$ in (3.18)(3.25), $i=1,2,3$, 4. Then, the following results hold.

(a) The products of $A_{i}$ and $B_{i}, i=1,2,3,4$, satisfy

$$
\begin{array}{lll}
A_{1} B_{1}=A B, & A_{1} B_{1} A_{1}=A B A, & B_{1} A_{1} B_{1}=B A B, \\
A_{2} B_{2}=A \widetilde{B}, & A_{2} B_{2} A_{2}=A \widetilde{B} A, & B_{2} A_{2} B_{2}=\widetilde{B} A \widetilde{B}, \\
A_{3} B_{3}=\widetilde{A} B, & A_{3} B_{3} A_{3}=\widetilde{A} B \widetilde{A}, & B_{3} A_{3} B_{3}=\widetilde{B A} B, \\
A_{4} B_{4}=\widetilde{A} \widetilde{B}, & A_{4} B_{4} A_{4}=\widetilde{A} \widetilde{B} \widetilde{A}, & B_{4} A_{4} B_{4}=\widetilde{B} \widetilde{A} \widetilde{B} .
\end{array}
$$

(b) There exists a unitary matrix $U$ such that

$$
\begin{aligned}
& A_{1}=U \operatorname{diag}\left(I_{k_{1}}, 0_{k_{2}}, I_{k_{3}}, 0_{k_{4}+k_{5}+k_{6}}\right) U^{*}, \quad B_{1}=U \operatorname{diag}\left(\left[\begin{array}{cc}
C^{2} & C S \\
C S & S^{2}
\end{array}\right], I_{k_{3}}, 0_{k_{4}+k_{5}+k_{6}}\right) U^{*}, \\
& A_{2}=U \operatorname{diag}\left(I_{k_{1}}, 0_{k_{2}+k_{3}}, I_{k_{4}}, 0_{k_{5}+k_{6}}\right) U^{*}, \quad B_{2}=U \operatorname{diag}\left(\left[\begin{array}{cc}
S^{2} & -C S \\
-C S & C^{2}
\end{array}\right], 0_{k_{3}}, I_{k_{4}}, 0_{k_{5}+k_{6}}\right) U^{*}, \\
& A_{3}=U \operatorname{diag}\left(0_{k_{1}}, I_{k_{2}}, 0_{k_{3}+k_{4}}, I_{k_{5}}, 0_{k_{6}}\right) U^{*}, B_{3}=U \operatorname{diag}\left(\left[\begin{array}{cc}
C^{2} & C S \\
C S & S^{2}
\end{array}\right], 0_{k_{3}+k_{4}}, I_{k_{5}}, 0_{k_{6}}\right) U^{*}, \\
& A_{4}=U \operatorname{diag}\left(0_{k_{1}}, I_{k_{2}}, 0_{k_{3}+k_{4}+k_{5}}, I_{k_{6}}\right) U^{*}, \quad B_{4}=U \operatorname{diag}\left(\left[\begin{array}{cc}
S^{2} & -C S \\
-C S & C^{2}
\end{array}\right], 0_{k_{3}+k_{4}+k_{5}}, I_{k_{6}}\right) U^{*},
\end{aligned}
$$




$$
\begin{aligned}
& A_{1}+B_{1}=U \operatorname{diag}\left(\left[\begin{array}{cc}
I_{k_{1}}+C^{2} & C S \\
C S & S^{2}
\end{array}\right], 2 I_{k_{3}}, 0_{k_{4}+k_{5}+k_{6}}\right) U^{*}, \\
& A_{1}-B_{1}=U \operatorname{diag}\left(\left[\begin{array}{cc}
S^{2} & -C S \\
-C S & -S^{2}
\end{array}\right], 0_{m-2 k_{1}}\right) U^{*}, \\
& A_{2}+B_{2}=U \operatorname{diag}\left(\left[\begin{array}{cc}
I_{k_{1}}+S^{2} & -C S \\
-C S & C^{2}
\end{array}\right], 0_{k_{3}}, 2 I_{k_{4}}, 0_{k_{5}+k_{6}}\right) U^{*}, \\
& A_{2}-B_{2}=U \operatorname{diag}\left(\left[\begin{array}{cc}
C^{2} & C S \\
C S & -C^{2}
\end{array}\right], 0_{m-2 k_{1}}\right) U^{*}, \\
& A_{3}+B_{3}=U \operatorname{diag}\left(\left[\begin{array}{cc}
C & C S \\
C S & I_{k_{2}}+S^{2}
\end{array}\right], 0_{k_{3}+k_{4}}, 2 I_{k_{5}}, 0_{k_{6}}\right) U^{*}, \\
& A_{3}-B_{3}=U \operatorname{diag}\left(\left[\begin{array}{cc}
-C^{2} & -C S \\
-C S & C^{2}
\end{array}\right], 0_{m-2 k_{1}}\right) U^{*}, \\
& A_{4}+B_{4}=U \operatorname{diag}\left(\left[\begin{array}{cc}
S^{2} & -C S \\
-C S & I_{k_{2}}+C^{2}
\end{array}\right], 0_{k_{3}+k_{4}+k_{5}}, 2 I_{k_{6}}\right) U^{*}, \\
& A_{4}-B_{4}=U \operatorname{diag}\left(\left[\begin{array}{cc}
-S^{2} & C S \\
C S & S^{2}
\end{array}\right], 0_{m-2 k_{1}}\right) U^{*}, \\
& A_{1}-A_{2}=U \operatorname{diag}\left(0_{2 k_{1}}, I_{k_{3}},-I_{k_{4}}, 0_{k_{5}+k_{6}}\right) U^{*}, A_{3}-A_{4}=U \operatorname{diag}\left(0_{2 k_{1}+k_{3}+k_{4}}, I_{k_{5}},-I_{k_{6}}\right) U^{*}, \\
& B_{1}-B_{3}=U \operatorname{diag}\left(0_{2 k_{1}}, I_{k_{3}}, 0_{k_{4}},-I_{k_{5}}, 0_{k_{6}}\right) U^{*}, B_{2}-B_{4}=U \operatorname{diag}\left(0_{2 k_{1}+k_{3}}, I_{k_{4}}, 0_{k_{5}},-I_{k_{6}}\right) U^{*}, \\
& A_{1}+A_{3}=U \operatorname{diag}\left(I_{2 k_{1}+k_{3}}, 0_{k_{4}}, I_{k_{5}}, 0_{k_{6}}\right) U^{*}, \quad B_{1}+B_{2}=U \operatorname{diag}\left(I_{2 k_{1}+k_{3}+k_{4}}, 0_{k_{5}+k_{6}}\right) U^{*}, \\
& A_{2}+A_{4}=U \operatorname{diag}\left(I_{2 k_{1}}, 0_{k_{3}}, I_{k_{4}}, 0_{k_{5}}, I_{k_{6}}\right) U^{*}, \quad B_{3}+B_{4}=U \operatorname{diag}\left(I_{2 k_{1}}, 0_{k_{3}+k_{4}}, I_{k_{5}+k_{6}}\right) U^{*}, \\
& A_{1}+A_{4}=B_{1}+B_{4}=U \operatorname{diag}\left(I_{2 k_{1}+k_{3}}, 0_{k_{4}+k_{5}}, I_{k_{6}}\right) U^{*}, \\
& A_{2}+A_{3}=B_{2}+B_{3}=U \operatorname{diag}\left(I_{2 k_{1}}, 0_{k_{3}}, I_{k_{4}+k_{5}}, 0_{k_{6}}\right) U^{*}, \\
& A_{1}+A_{2}+A_{3}+A_{4}=B_{1}+B_{2}+B_{3}+B_{4}=I_{m}+U \operatorname{diag}\left(I_{2 k_{1}}, 0_{m-2 k_{1}}\right) U^{*} .
\end{aligned}
$$

In particular,

$$
\begin{aligned}
& A-A_{2}=B-B_{3}=U \operatorname{diag}\left(0_{2 k_{1}}, I_{k_{3}}, 0_{k_{4}+k_{5}+k_{6}}\right) U^{*}=P_{\mathcal{M} \cap \mathcal{N}}, \\
& A-A_{1}=\widetilde{B}-B_{4}=U \operatorname{diag}\left(0_{2 k_{1}+k_{3}}, I_{k_{4}}, 0_{k_{5}+k_{6}}\right) U^{*}=P_{\mathcal{M} \cap \mathcal{N}^{\perp}}, \\
& \widetilde{A}-A_{4}=B-B_{1}=U \operatorname{diag}\left(0_{2 k_{1}+k_{3}+k_{4}}, I_{k_{5}}, 0_{k_{6}}\right) U^{*}=P_{\mathcal{M} \perp \perp \mathcal{N},}, \\
& \widetilde{A}-A_{3}=\widetilde{B}-B_{2}=U \operatorname{diag}\left(0_{m-k_{6}}, I_{k_{6}}\right) U^{*}=P_{\mathcal{M}^{\perp} \cap \mathcal{N}^{\perp} .} .
\end{aligned}
$$

(c) The Moore-Penrose inverses of the matrices in Corollary 3.6(b) are given by

$$
\begin{aligned}
& \left(A_{1}+B_{1}\right)^{\dagger}=U \operatorname{diag}\left(\left[\begin{array}{cc}
I_{k_{1}} & -C S^{-1} \\
-C S^{-1} & S^{-2}+C^{2} S^{-2}
\end{array}\right], 2^{-1} I_{k_{3}}, 0_{k_{4}+k_{5}+k_{6}}\right) U^{*}, \\
& \left(A_{1}-B_{1}\right)^{\dagger}=U \operatorname{diag}\left(\left[\begin{array}{cc}
I_{k_{1}} & -C S^{-1} \\
-C S^{-1} & -I_{k_{2}}
\end{array}\right], 0_{m-2 k_{1}}\right) U^{*}, \\
& \left(A_{2}+B_{2}\right)^{\dagger}=U \operatorname{diag}\left(\left[\begin{array}{cc}
I_{k_{1}} & C^{-1} S \\
C^{-1} S & C^{-2}+C^{-2} S^{2}
\end{array}\right], 0_{k_{3}}, 2^{-1} I_{k_{4}}, 0_{k_{5}+k_{6}}\right) U^{*}, \\
& \left(A_{2}-B_{2}\right)^{\dagger}=U \operatorname{diag}\left(\left[\begin{array}{cc}
I_{k_{1}} & C^{-1} S \\
C^{-1} S & -I_{k_{2}}
\end{array}\right], 0_{m-2 k_{1}}\right) U^{*}, \\
& \left(A_{3}+B_{3}\right)^{\dagger}=U \operatorname{diag}\left(\left[\begin{array}{cc}
C^{-2}+C^{-2} S^{2} & -C^{-1} S \\
-C S^{-1} & I_{k_{2}}
\end{array}\right], 0_{k_{3}+k_{4}}, 2^{-1} I_{k_{5}}, 0_{k_{6}}\right) U^{*},
\end{aligned}
$$




$$
\begin{aligned}
& \left(A_{3}-B_{3}\right)^{\dagger}=U \operatorname{diag}\left(\left[\begin{array}{cc}
-I_{k_{1}} & -C^{-1} S \\
-C^{-1} S & I_{k_{2}}
\end{array}\right], 0_{m-2 k_{1}}\right) U^{*}, \\
& \left(A_{4}+B_{4}\right)^{\dagger}=U \operatorname{diag}\left(\left[\begin{array}{cc}
S^{-2}+C^{2} S^{-2} & C S^{-1} \\
C S^{-1} & I_{k_{2}}
\end{array}\right], 0_{k_{3}+k_{4}+k_{5}}, 2^{-1} I_{k_{6}}\right) U^{*}, \\
& \left(A_{4}-B_{4}\right)^{\dagger}=U \operatorname{diag}\left(\left[\begin{array}{cc}
-I_{k_{1}} & C S^{-1} \\
C S^{-1} & I_{k_{2}}
\end{array}\right], 0_{m-2 k_{1}}\right) U^{*} .
\end{aligned}
$$

(d) The following dimension identities hold

$$
\begin{aligned}
& \operatorname{dim}\left(\mathcal{S}_{1}\right)=\operatorname{dim}\left(\mathcal{T}_{1}\right)=r\left(A_{1}\right)=r\left(B_{1}\right)=r(A B), \operatorname{dim}\left(\mathcal{S}_{2}\right)=\operatorname{dim}\left(\mathcal{T}_{2}\right)=r\left(A_{2}\right)=r\left(B_{2}\right)=r(A \widetilde{B}), \\
& \operatorname{dim}\left(\mathcal{S}_{3}\right)=\operatorname{dim}\left(\mathcal{T}_{3}\right)=r\left(A_{3}\right)=r\left(B_{3}\right)=(\widetilde{A} B), \quad \operatorname{dim}\left(\mathcal{S}_{4}\right)=\operatorname{dim}\left(\mathcal{T}_{4}\right)=r\left(A_{4}\right)=r\left(B_{4}\right)=r(\widetilde{A} \widetilde{B}) .
\end{aligned}
$$

(e) The following orthogonal projector identities hold

$$
P_{\mathcal{S}_{1}}=A_{1}, P_{\mathcal{T}_{1}}=B_{1}, P_{\mathcal{S}_{2}}=A_{2}, P_{\mathcal{T}_{2}}=B_{2}, P_{\mathcal{S}_{3}}=A_{3}, P_{\mathcal{T}_{3}}=B_{3}, P_{\mathcal{S}_{4}}=A_{4}, P_{\mathcal{T}_{4}}=B_{4} .
$$

(f) The following intersection identities for subspaces hold

$$
\mathcal{S}_{1} \cap \mathcal{T}_{1}=\mathcal{M} \cap \mathcal{N}, \quad \mathcal{S}_{2} \cap \mathcal{T}_{2}=\mathcal{M} \cap \mathcal{N}^{\perp}, \quad \mathcal{S}_{3} \cap \mathcal{T}_{3}=\mathcal{M}^{\perp} \cap \mathcal{N}, \quad \mathcal{S}_{4} \cap \mathcal{T}_{4}=\mathcal{M}^{\perp} \cap \mathcal{N}^{\perp} .
$$

Although the results in this section are established in the well-known finite-dimensional complex vector space, most conclusions are obtained only by the usual sum and intersection operations of subspaces. Thus, they can easily be extended to subspaces in various general algebraic structures in which subspaces and their orthogonal complements can be defined.

\section{Formulas for orthogonal projectors onto the intersections of two subspaces}

Finding explicit expressions of the orthogonal projector onto the intersection of two linear subspaces is a fundamental and interesting problem in linear algebra, which was considered by some authors and many results were obtained, see e.g., in [31, 82, 109, 116, 134, 139]. Although Theorem 3.4(e) give decomposition expressions of the four orthogonal projectors, we cannot clarify explicit contributions of $A$ and $B$ in these representations. In this section, we give a family of known or novel formulas composed by algebraic operations of $A$ and $B$ for representing the orthogonal projectors onto the intersection of two linear subspaces.

Theorem 4.1. Let $\mathcal{M}$ and $\mathcal{N}$ be two linear subspaces of $\mathbb{C}^{m}, A, B, \widetilde{A}$, and $\widetilde{B}$ be as given in (3.2), $\mathcal{S}_{1}$ and $\mathcal{T}_{1}$ be as given in (3.18), and $A_{i}$ and $B_{i}$ be as given in (3.22)-(3.25), $i=1, \ldots, 4$. Then, the orthogonal projector $P_{\mathcal{M} \cap \mathcal{N}}$ onto the intersection $\mathcal{M} \cap \mathcal{N}$ can be written in the following forms

$$
\begin{aligned}
P_{\mathcal{M} \cap \mathcal{N}} & =A-(A \widetilde{B})(A \widetilde{B})^{\dagger}=A-A_{2} \quad\left(=B-(B \widetilde{A})(B \widetilde{A})^{\dagger}=B-B_{3}\right) \\
& =\frac{1}{2}\left(A+B-A_{2}-B_{3}\right) \\
& =A-A(\widetilde{B} A \widetilde{B})^{\dagger} A \quad\left(=B-B(\widetilde{A} B \widetilde{A})^{\dagger} B\right) \\
& =A B A-A B(\widetilde{A} B \widetilde{A})^{\dagger} B A \quad\left(=B A B-B A(\widetilde{B} A \widetilde{B})^{\dagger} A B\right) \\
& =2 A(A+B)^{\dagger} B=2(A: B) \quad\left(=2 B(A+B)^{\dagger} A=2(B: A)\right) \\
& =A(A+B)^{\dagger} B+B(A+B)^{\dagger} A \\
& =2 A B(A+B)^{\dagger} A B \quad\left(=2 B A(A+B)^{\dagger} B A\right)
\end{aligned}
$$


$=A B(A+B)^{\dagger} A B+B A(A+B)^{\dagger} B A$

$=2 A-2 A(A+B)^{\dagger} A \quad\left(=2 B-2 B(A+B)^{\dagger} B\right)$

$=A+B-A(A+B)^{\dagger} A-B(A+B)^{\dagger} B$

$=2 A B A-2 A B(A+B)^{\dagger} B A \quad\left(=2 B A B-2 B A(A+B)^{\dagger} A B\right)$

$=A B A+B A B-A B(A+B)^{\dagger} B A-B A(A+B)^{\dagger} A B$

$=\left[A(A+B)^{\dagger} A\right]^{\dagger}-A \quad\left(=\left[B(A+B)^{\dagger} B\right]^{\dagger}-B\right)$

$=\frac{1}{2}\left[A(A+B)^{\dagger} A\right]^{\dagger}+\frac{1}{2}\left[B(A+B)^{\dagger} B\right]^{\dagger}-\frac{1}{2}(A+B)$

$=A\left[B(A+B)^{\dagger} B\right]^{\dagger} A-A B A \quad\left(=B\left[A(A+B)^{\dagger} A\right]^{\dagger} B-B A B\right)$

$=\frac{1}{2} A\left[B(A+B)^{\dagger} B\right]^{\dagger} A+\frac{1}{2} B\left[A(A+B)^{\dagger} A\right]^{\dagger} B-\frac{1}{2}(A B A+B A B)$

$=(A+B)(A+B)^{\dagger}-(A-B)(A-B)^{\dagger}$

$=\left[I_{m}-(A-B)(A-B)^{\dagger}\right] A \quad\left(=\left[I_{m}-(A-B)(A-B)^{\dagger}\right] B\right)$

$=\frac{1}{2}\left[I_{m}-(A-B)(A-B)^{\dagger}\right](A+B)$

$=(A+B)(A+B)^{\dagger}-(A \widetilde{B})^{\dagger}-(B \widetilde{A})^{\dagger}$

$=\left[I_{m}-(A \widetilde{B})^{\dagger}-(B \widetilde{A})^{\dagger}\right] A \quad\left(=\left[I_{m}-(A \widetilde{B})^{\dagger}-(B \widetilde{A})^{\dagger}\right] B\right)$

$=\frac{1}{2}\left[I_{m}-(A \widetilde{B})^{\dagger}-(B \widetilde{A})^{\dagger}\right](A+B)$

$=(A+B)(A+B)^{\dagger}-A_{2}-A_{3} \quad\left(=(A+B)(A+B)^{\dagger}-B_{2}-B_{3}\right)$

$=(A+B)(A+B)^{\dagger}-\frac{1}{2}\left(A_{2}+A_{3}+B_{2}+B_{3}\right)$

$=A-A(A-B)^{\dagger} A \quad\left(=B-B(B-A)^{\dagger} B\right)$

$=\frac{1}{2}\left[A+B-A(A-B)^{\dagger} A-B(B-A)^{\dagger} B\right]$

$=A B A-A B(B-A)^{\dagger} B A \quad\left(=B A B-B A(A-B)^{\dagger} A B\right)$

$=\frac{1}{2}\left[A B A+B A B(B-A)^{\dagger} B A-B A(A-B)^{\dagger} A B\right]$

$=I_{m}-(\widetilde{A}+\widetilde{B})(\widetilde{A}+\widetilde{B})^{\dagger}=I_{m}-\left(2 I_{m}-A-B\right)\left(2 I_{m}-A-B\right)^{\dagger}$

$=I_{m}-\left(I_{m}-A B\right)\left(I_{m}-A B\right)^{\dagger} \quad\left(=I_{m}-\left(I_{m}-B A\right)\left(I_{m}-B A\right)^{\dagger}\right)$

$=I_{m}-\left(2 I_{m}-A B-B A\right)\left(2 I_{m}-A B-B A\right)^{\dagger}$

$=2 A(A B+B A)^{\dagger} A \quad\left(=2 B(A B+B A)^{\dagger} B\right)$

$=A(A B+B A)^{\dagger} A+B(A B+B A)^{\dagger} B$

$=2 A B(A B+B A)^{\dagger} B A \quad\left(=2 B A(A B+B A)^{\dagger} A B\right)$

$=A B(A B+B A)^{\dagger} B A+B A(A B+B A)^{\dagger} A B$

$=2(A B)^{\dagger}-2 B(A B+B A)^{\dagger} A \quad\left(=2(B A)^{\dagger}-2 A(A B+B A)^{\dagger} B\right)$

$=(A B)^{\dagger}+(B A)^{\dagger}-A(A B+B A)^{\dagger} B-B(A B+B A)^{\dagger} A$

$=2 A B-2 A B(A B+B A)^{\dagger} A B \quad\left(=2 B A-2 B A(A B+B A)^{\dagger} B A\right)$

$=A B+B A-A B(A B+B A)^{\dagger} A B-B A(A B+B A)^{\dagger} B A$

$=(A B+B A)(A B+B A)^{\dagger}-(A B-B A)(A B-B A)^{\dagger}$ 


$$
\begin{aligned}
& =I_{m}-\left(I_{m}-A B A\right)\left(I_{m}-A B A\right)^{\dagger} \quad\left(=I_{m}-\left(I_{m}-B A B\right)\left(I_{m}-B A B\right)^{\dagger}\right) \\
& =I_{m}-\left(2 I_{m}-A B A-B A B\right)\left(2 I_{m}-A B A-B A B\right)^{\dagger} \\
& =2(A B A)^{\dagger}-2 A(A B A+B A B)^{\dagger} A \quad\left(=2(B A B)^{\dagger}-2 B(A B A+B A B)^{\dagger} B\right) \\
& =(A B A)^{\dagger}+(B A B)^{\dagger}-A(A B A+B A B)^{\dagger} A-B(A B A+B A B)^{\dagger} B \\
& =2 A(B A B)^{\dagger} A-2 A B(A B A+B A B)^{\dagger} B A\left(=2 B(A B A)^{\dagger} B-2 B A(A B A+B A B)^{\dagger} A B\right) \\
& =A(B A B)^{\dagger} A+B(A B A)^{\dagger} B-A B(A B A+B A B)^{\dagger} B A-B A(A B A+B A B)^{\dagger} A B \\
& =2 A(A B A+B A B)^{\dagger} B \quad\left(=2 B(A B A+B A B)^{\dagger} A\right) \\
& =A(A B A+B A B)^{\dagger} B+B(A B A+B A B)^{\dagger} A \\
& =2 A B(A B A+B A B)^{\dagger} A B \quad\left(=2 B A(A B A+B A B)^{\dagger} B A\right) \\
& =A B(A B A+B A B)^{\dagger} A B+B A(A B A+B A B)^{\dagger} B A \\
& =2 A B A(A B A+B A B)^{\dagger} B A B \quad\left(=2 B A B(A B A+B A B)^{\dagger} A B A\right) \\
& =A B A(A B A+B A B)^{\dagger} B A B+B A B(A B A+B A B)^{\dagger} A B A \\
& =2 A B A-2 A B A(A B A+B A B)^{\dagger} A B A\left(=2 B A B-2 B A B(A B A+B A B)^{\dagger} B A B\right) \\
& =A B A+B A B-A B A(A B A+B A B)^{\dagger} A B A-B A B(A B A+B A B)^{\dagger} B A B \\
& =(A B)(A B)^{\dagger}-(A B \widetilde{A})(A B \widetilde{A})^{\dagger} \quad\left(=(B A)(B A)^{\dagger}-(B A \widetilde{B})(B A \widetilde{B})^{\dagger}\right) \\
& =\frac{1}{2}(A B A+B A B)-\frac{1}{2}[A B A, B A B, 0] \\
& =
\end{aligned}
$$$$
=-2\left[0,0, I_{m}\right]\left[\begin{array}{ccc}
A B A & 0 & I_{m} \\
0 & B A B & I_{m} \\
I_{m} & I_{m} & 0
\end{array}\right]^{\dagger}\left[\begin{array}{c}
0 \\
0 \\
I_{m}
\end{array}\right]
$$$$
=2 A_{1}\left(A_{1}+B_{1}\right)^{\dagger} B_{1}=2\left(A_{1}: B_{1}\right) \quad\left(=2 B_{1}\left(A_{1}+B_{1}\right)^{\dagger} A_{1}=2\left(B_{1}: A_{1}\right)\right)
$$$$
=A_{1}\left(A_{1}+B_{1}\right)^{\dagger} B_{1}+B_{1}\left(A_{1}+B_{1}\right)^{\dagger} A_{1}
$$$$
=2 A_{1}-2 A_{1}\left(A_{1}+B_{1}\right)^{\dagger} A_{1} \quad\left(=2 B_{1}-2 B_{1}\left(A_{1}+B_{1}\right)^{\dagger} B_{1}\right)
$$$$
=A_{1}+B_{1}-A_{1}\left(A_{1}+B_{1}\right)^{\dagger} A_{1}-B_{1}\left(A_{1}+B_{1}\right)^{\dagger} B_{1}
$$ 


$$
\begin{aligned}
& =\left(A_{1}+B_{1}\right)\left(A_{1}+B_{1}\right)^{\dagger}-\left(A_{1}-B_{1}\right)\left(A_{1}-B_{1}\right)^{\dagger} \\
& =\left[I_{m}-\left(A_{1}-B_{1}\right)\left(A_{1}-B_{1}\right)^{\dagger}\right] A_{1} \quad\left(=\left[I_{m}-\left(A_{1}-B_{1}\right)\left(A_{1}-B_{1}\right)^{\dagger}\right] B_{1}\right) \\
& =\frac{1}{2}\left[I_{m}-\left(A_{1}-B_{1}\right)\left(A_{1}-B_{1}\right)^{\dagger}\right]\left(A_{1}+B_{1}\right) \\
& =\left(A_{1}+B_{1}\right)\left(A_{1}+B_{1}\right)^{\dagger}-\left(A_{1} \widetilde{B}_{1}\right)^{\dagger}-\left(B_{1} \widetilde{A}_{1}\right)^{\dagger} \\
& =\left(A_{1}+B_{1}\right)\left(A_{1}+B_{1}\right)^{\dagger}-\left(A_{1} \widetilde{B}_{1}\right)\left(A_{1} \widetilde{B}_{1}\right)^{\dagger}-\left(\widetilde{A}_{1} B_{1}\right)\left(\widetilde{A}_{1} B_{1}\right)^{\dagger} \\
& =\left(A_{1}+B_{1}\right)\left(A_{1}+B_{1}\right)^{\dagger}-\left(B_{1} \widetilde{A}_{1}\right)\left(B_{1} \widetilde{A}_{1}\right)^{\dagger}-\left(\widetilde{B}_{1} A_{1}\right)\left(\widetilde{B}_{1} A_{1}\right)^{\dagger} \\
& =A_{1}-\left(A_{1} \widetilde{B}_{1}\right)\left(A_{1} \widetilde{B}_{1}\right)^{\dagger} \quad\left(=B_{1}-\left(B_{1} \widetilde{A}_{1}\right)\left(B_{1} \widetilde{A}_{1}\right)^{\dagger}\right) \\
& =\frac{1}{2}\left(A_{1}+B_{1}\right)-\frac{1}{2}\left(A_{1} \widetilde{B}_{1}\right)\left(A_{1} \widetilde{B}_{1}\right)^{\dagger}-\frac{1}{2}\left(B_{1} \widetilde{A}_{1}\right)\left(B_{1} \widetilde{A}_{1}\right)^{\dagger} \\
& =A_{1}-A_{1}\left(A_{1}-B_{1}\right)^{\dagger} A_{1} \quad\left(=B_{1}-B_{1}\left(B_{1}-A_{1}\right)^{\dagger} B_{1}\right) \\
& =\frac{1}{2}\left(A_{1}+B_{1}\right)-\frac{1}{2} A_{1}\left(A_{1}-B_{1}\right)^{\dagger} A_{1}-\frac{1}{2} B_{1}\left(B_{1}-A_{1}\right)^{\dagger} B_{1} \\
& =I_{m}-\left(2 I_{m}-A_{1}-B_{1}\right)\left(2 I_{m}-A_{1}-B_{1}\right)^{\dagger} \\
& =\frac{1}{2}\left(A_{1}+B_{1}\right)-\frac{1}{2}\left[A_{1}, B_{1}, 0\right]\left[\begin{array}{ccc}
A_{1} & 0 & I_{m} \\
0 & B_{1} & I_{m} \\
I_{m} & I_{m} & 0
\end{array}\right]^{\dagger}\left[\begin{array}{c}
A_{1} \\
B_{1} \\
0
\end{array}\right] \\
& =-2\left[0,0, I_{m}\right]\left[\begin{array}{ccc}
A_{1} & 0 & I_{m} \\
0 & B_{1} & I_{m} \\
I_{m} & I_{m} & 0
\end{array}\right]^{\dagger}\left[\begin{array}{c}
0 \\
0 \\
I_{m}
\end{array}\right] \\
& =\lim _{k \rightarrow \infty}(A+B)^{k} / 2^{k} \\
& =\lim _{k \rightarrow \infty}(A B)^{k} \quad\left(=\lim _{k \rightarrow \infty}(B A)^{k}\right) \quad([83,101]) \\
& =\lim _{k \rightarrow \infty}(A B A)^{k} \quad\left(=\lim _{k \rightarrow \infty}(B A B)^{k}\right) \\
& =\lim _{k \rightarrow \infty}(A B+B A)^{k} / 2^{k} \\
& =\lim _{k \rightarrow \infty}(A B A+B A B)^{k} / 2^{k} \\
& =A-\lim _{k \rightarrow \infty}\left[A(A+B)^{\dagger} A\right]^{k} \quad\left(=B-\lim _{k \rightarrow \infty}\left[B(A+B)^{\dagger} B\right]^{k}\right) \\
& =\lim _{k \rightarrow \infty}\left(A_{1}+B_{1}\right)^{k} / 2^{k} \\
& =I_{m}-\lim _{k \rightarrow \infty}\left(2 I_{m}-A-B\right)^{\frac{1}{k}} \\
& =A-\lim _{k \rightarrow \infty}(A \widetilde{B} A)^{\frac{1}{k}} \quad\left(=B-\lim _{k \rightarrow \infty}(B \widetilde{A} B)^{\frac{1}{k}}\right) \\
& =I_{m}-\lim _{k \rightarrow \infty}\left(I_{m}-A B A\right)^{\frac{1}{k}} \quad\left(=I_{m}-\lim _{k \rightarrow \infty}\left(I_{m}-B A B\right)^{\frac{1}{k}}\right) \\
& =I_{m}-\lim _{k \rightarrow \infty}\left(2 I_{m}-A B A-B A B\right)^{\frac{1}{k}} .
\end{aligned}
$$

In consequence, the following statements are equivalent:
$\langle 1\rangle \mathcal{M} \cap \mathcal{N}=\{0\}$.
$\langle 2\rangle-\langle 41\rangle$ in Corollary 2.13(a).
$\langle 42\rangle r[A, B]=r(A)+r(B)$.
〈43〉 $r(A \widetilde{B})=r(A)$ and $/$ or $r(B \widetilde{A})=r(B)$.
444〉 $\mathscr{R}(A \widetilde{B})=\mathscr{R}(A)$ and/or $\mathscr{R}(B \widetilde{A})=\mathscr{R}(B)$.
$\langle 45\rangle \mathcal{M}=\mathcal{S}_{2}$ and/or $\mathcal{N}=\mathcal{T}_{3}$. 
$\langle 46\rangle A=A_{2}$ and $/$ or $B=B_{3}$.

$\langle 47\rangle A+B=A_{2}+B_{3}$.

$\langle 48\rangle A(\widetilde{B} A \widetilde{B})^{\dagger} A=A$ and $/$ or $B(\widetilde{A} B \widetilde{A})^{\dagger} B=B$.

$\langle 49\rangle A B(\widetilde{A} B \widetilde{A})^{\dagger} B A=A B A$ and /or $B A(\widetilde{B} A \widetilde{B})^{\dagger} A B=B A B$.

$\langle 50\rangle A(A+B)^{\dagger} B=0$ and $/$ or $B(A+B)^{\dagger} A=0$.

$\langle 51\rangle A(A+B)^{\dagger} B+B(A+B)^{\dagger} A=0$.

〈52) $A B(A+B)^{\dagger} A B=0$ and/or $B A(A+B)^{\dagger} B A=0$.

〈53〉 $A B(A+B)^{\dagger} A B+B A(A+B)^{\dagger} B A=0$.

〈54〉 $A B(A+B)^{\dagger} B A=A B A$ and/or $B A(A+B)^{\dagger} A B=B A B$.

$\langle 55\rangle A B(A+B)^{\dagger} B A+B A(A+B)^{\dagger} A B=A B A+B A B$.

$\langle 56\rangle\left[A(A+B)^{\dagger} A\right]^{\dagger}=A$ and $/$ or $\left[B(A+B)^{\dagger} B\right]^{\dagger}=B$.

$\langle 57\rangle\left[A(A+B)^{\dagger} A\right]^{\dagger}+\left[B(A+B)^{\dagger} B\right]^{\dagger}=A+B$.

$\langle 58\rangle A\left[B(A+B)^{\dagger} B\right]^{\dagger} A=A B A$ and/or $B\left[A(A+B)^{\dagger} A\right]^{\dagger}=B A B$.

$\langle 59\rangle A\left[B(A+B)^{\dagger} B\right]^{\dagger} A+B\left[A(A+B)^{\dagger} A\right]^{\dagger} B=A B A+B A B$.

$\langle 60\rangle(A+B)(A+B)^{\dagger}=(A-B)(A-B)^{\dagger}$, i.e., $\mathscr{R}(A+B)=\mathscr{R}(A-B)$.

$\langle 61\rangle \mathscr{R}(A) \subseteq \mathscr{R}(A-B)$ and $/$ or $\mathscr{R}(B) \subseteq \mathscr{R}(A-B)$.

$\langle 62\rangle(A+B)(A+B)^{\dagger}=(A \widetilde{B})^{\dagger}+(B \widetilde{A})^{\dagger}$.

$\langle 63\rangle\left[(A \widetilde{B})^{\dagger}+(B \widetilde{A})^{\dagger}\right] A=A$ and $/$ or $\left[(A \widetilde{B})^{\dagger}+(B \widetilde{A})^{\dagger}\right] B=B$.

$\langle 64\rangle\left[(A \widetilde{B})^{\dagger}+(B \widetilde{A})^{\dagger}\right](A+B)=A+B$.

$\langle 65\rangle(A+B)(A+B)^{\dagger}=A_{2}+A_{3}$ and $/$ or $(A+B)(A+B)^{\dagger}=B_{2}+B_{3}$.

$\langle 66\rangle 2(A+B)(A+B)^{\dagger}=A_{2}+A_{3}+B_{2}+B_{3}$.

$\langle 67\rangle A(A-B)^{\dagger} A=A$ and $/$ or $B(B-A)^{\dagger} B=B$.

<68 $A(A-B)^{\dagger} A+B(B-A)^{\dagger} B=A+B$.

<69 $A B(B-A)^{\dagger} B A=A B A$ and $/$ or $B A(A-B)^{\dagger} A B=B A B$.

$\langle 70\rangle A B(B-A)^{\dagger} B A+B A(A-B)^{\dagger} A B=A B A+B A B$.

$\langle 71\rangle A+B \prec 2 I_{m}$.

$\langle 72\rangle r\left(I_{m}-A B\right)=r\left(I_{m}-B A\right)=m$.

$\langle 73\rangle A B+B A \prec 2 I_{m}$.

$\langle 74\rangle A(A B+B A)^{\dagger} A=0$ and $/$ or $B(A B+B A)^{\dagger} B=0$.

<75〉 $A(A B+B A)^{\dagger} A+B(A B+B A)^{\dagger} B=0$.

$\langle 76\rangle A B(A B+B A)^{\dagger} B A=0$ and/or $B A(A B+B A)^{\dagger} A B=0$.

$\langle 77\rangle A B(A B+B A)^{\dagger} B A+B A(A B+B A)^{\dagger} A B=0$.

$\langle 78\rangle(A B)^{\dagger}=2 B(A B+B A)^{\dagger} A$ and $/$ or $(B A)^{\dagger}=2 A(A B+B A)^{\dagger} B$.

$\langle 79\rangle(A B)^{\dagger}+(B A)^{\dagger}=A(A B+B A)^{\dagger} B-B(A B+B A)^{\dagger} A$.

$\langle 80\rangle A B(A B+B A)^{\dagger} A B=A B$ and/or $B A(A B+B A)^{\dagger} B A=B A$.

<81 $A B(A B+B A)^{\dagger} A B-B A(A B+B A)^{\dagger} B A=A B+B A$.

$\langle 82\rangle \mathscr{R}(A B+B A)=\mathscr{R}(A B-B A)$.

$\langle 83\rangle A B A \prec I_{m}$ and/or $B A B \prec I_{m}$.

$\langle 84\rangle A B A+B A B \prec 2 I_{m}$.

$\langle 85\rangle(A B A)^{\dagger}=A(A B A+B A B)^{\dagger} A$ and $/$ or $(B A B)^{\dagger}=B(A B A+B A B)^{\dagger} B$.

$\langle 86\rangle(A B A)^{\dagger}+(B A B)^{\dagger}=A(A B A+B A B)^{\dagger} A+B(A B A+B A B)^{\dagger} B$.

<87 $A(B A B)^{\dagger} A=A B(A B A+B A B)^{\dagger} B A$ and $/$ or $B(A B A)^{\dagger} B=B A(A B A+B A B)^{\dagger} A B$.

<88 $A(B A B)^{\dagger} A+B(A B A)^{\dagger} B=A B(A B A+B A B)^{\dagger} B A+B A(A B A+B A B)^{\dagger} A B$.

<89 $A(A B A+B A B)^{\dagger} B=0$ and/or $B(A B A+B A B)^{\dagger} A=0$.

$\langle 90\rangle A(A B A+B A B)^{\dagger} B+B(A B A+B A B)^{\dagger} A=0$.

〈91) $A B(A B A+B A B)^{\dagger} A B=0$ and $/$ or $B A(A B A+B A B)^{\dagger} B A=0$.

〈92 $A B(A B A+B A B)^{\dagger} A B+B A(A B A+B A B)^{\dagger} B A=0$.

$\langle 93\rangle A B A(A B A+B A B)^{\dagger} B A B=0$ and/or $B A B(A B A+B A B)^{\dagger} A B A=0$.

〈94) $A B A(A B A+B A B)^{\dagger} B A B+B A B(A B A+B A B)^{\dagger} A B A=0$.

〈95) $A B A(A B A+B A B)^{\dagger} A B A=A B A$ and $/$ or $B A B(A B A+B A B)^{\dagger} B A B=B A B$.

(96) $A B A(A B A+B A B)^{\dagger} A B A+B A B(A B A+B A B)^{\dagger} B A B=A B A+B A B$. 
$\langle 97\rangle(A B \widetilde{A})(A B \widetilde{A})^{\dagger}=(A B)(A B)^{\dagger}$ and $/$ or $(B A \widetilde{B})(B A \widetilde{B})^{\dagger}=(B A)(B A)^{\dagger}$.

$\langle 98\rangle A(A-B A B)^{\dagger} A=A$ and/or $B(B-A B A)^{\dagger} B=B$.

$\langle 99\rangle A B(B-A B A)^{\dagger} B A=A B A$ and/or $B A(A-B A B)^{\dagger} A B=B A B$.

$\langle 100\rangle \mathscr{R}(A B)=\mathscr{R}(A B \widetilde{A})$ and $/$ or $\mathscr{R}(B A)=\mathscr{R}(B A \widetilde{B})$.

$\langle 101\rangle A(A-B A B)^{\dagger} A=A$ and $/$ or $B(B-A B A)^{\dagger} B=A$.

$\langle 102\rangle A(A-B A B)^{\dagger} A+B(B-A B A)^{\dagger} B=A+B$.

$\langle 103\rangle A B(B-A B A)^{\dagger} B A=A B A$ and/or $B A(A-B A B)^{\dagger} A B=B A B$.

$\langle 104\rangle A B(B-A B A)^{\dagger} B A+B A(A-B A B)^{\dagger} A B=A B A+B A B$.

$\langle 105\rangle A(B A \widetilde{B}+\widetilde{B} A B)^{\dagger} A=2 A B(A B)^{\dagger}$ and/or $B(A B \widetilde{A}+\widetilde{A} B A)^{\dagger} B=2 B A(B A)^{\dagger}$.

$\langle 106\rangle A B(A B \widetilde{A}+\widetilde{A} B A)^{\dagger} B A=2 A B A$ and $/$ or $B A(B A \widetilde{B}+\widetilde{B} A B)^{\dagger} A B=2 B A B$.

$\langle 107\rangle[A, B, 0]\left[\begin{array}{ccc}A & 0 & I_{m} \\ 0 & B & I_{m} \\ I_{m} & I_{m} & 0\end{array}\right]^{\dagger}\left[\begin{array}{l}A \\ B \\ 0\end{array}\right]=A+B$.

$\langle 108\rangle\left[0,0, I_{m}\right]\left[\begin{array}{ccc}A & 0 & I_{m} \\ 0 & B & I_{m} \\ I_{m} & I_{m} & 0\end{array}\right]^{\dagger}\left[\begin{array}{c}0 \\ 0 \\ I_{m}\end{array}\right]=0$.

$\langle 109\rangle[A B A, B A B, 0]\left[\begin{array}{ccc}A B A & 0 & I_{m} \\ 0 & B A B & I_{m} \\ I_{m} & I_{m} & 0\end{array}\right]^{\dagger}\left[\begin{array}{c}A B A \\ B A B \\ 0\end{array}\right]=A B A+B A B$.

$\langle 110\rangle\left[0,0, I_{m}\right]\left[\begin{array}{ccc}A B A & 0 & I_{m} \\ 0 & B A B & I_{m} \\ I_{m} & I_{m} & 0\end{array}\right]^{\dagger}\left[\begin{array}{c}0 \\ 0 \\ I_{m}\end{array}\right]=0$.

$\langle 111\rangle \mathcal{S}_{1} \cap \mathcal{T}_{1}=\{0\}$, i.e., $\mathscr{R}(A B) \cap \mathscr{R}(B A)=\{0\}$.

$\langle 112\rangle r\left[A_{1}, B_{1}\right]=r\left(A_{1}\right)+r\left(B_{1}\right)$. i.e., $r[A B, B A]=2 r(A B)$.

$\langle 113\rangle A_{1}\left(A_{1}+B_{1}\right)^{\dagger} B_{1}=0$ and/or $B_{1}\left(A_{1}+B_{1}\right)^{\dagger} A_{1}=0$.

$\langle 114\rangle A_{1}\left(A_{1}+B_{1}\right)^{\dagger} B_{1}+B_{1}\left(A_{1}+B_{1}\right)^{\dagger} A_{1}=0$.

$\langle 115\rangle A_{1}\left(A_{1}+B_{1}\right)^{\dagger} A_{1}=A_{1}$ and/or $B_{1}\left(A_{1}+B_{1}\right)^{\dagger} B_{1}=B_{1}$.

$\langle 116\rangle A_{1}\left(A_{1}+B_{1}\right)^{\dagger} A_{1}+B_{1}\left(A_{1}+B_{1}\right)^{\dagger} B_{1}=A_{1}+B_{1}$.

$\langle 117\rangle \mathscr{R}\left(A_{1}+B_{1}\right)=\mathscr{R}\left(A_{1}-B_{1}\right)$.

$\langle 118\rangle\left(A_{1}+B_{1}\right)\left(A_{1}+B_{1}\right)^{\dagger}=\left(A_{1} \widetilde{B}_{1}\right)^{\dagger}+\left(B_{1} \widetilde{A}_{1}\right)^{\dagger}$.

$\langle 119\rangle\left(A_{1}+B_{1}\right)\left(A_{1}+B_{1}\right)^{\dagger}=\left(A_{1} \widetilde{B}_{1}\right)\left(A_{1} \widetilde{B}_{1}\right)^{\dagger}+\left(\widetilde{A}_{1} B_{1}\right)\left(\widetilde{A}_{1} B_{1}\right)^{\dagger}$ and $/$ or $\left(A_{1}+B_{1}\right)\left(A_{1}+B_{1}\right)^{\dagger}=\left(B_{1} \widetilde{A}_{1}\right)\left(B_{1} \widetilde{A}_{1}\right)^{\dagger}+$ $\left(\widetilde{B}_{1} A_{1}\right)\left(\widetilde{B}_{1} A_{1}\right)^{\dagger}$.

$\langle 120\rangle\left(A_{1} \widetilde{B}_{1}\right)\left(A_{1} \widetilde{B}_{1}\right)^{\dagger}=A_{1}$ and /or $\left(B_{1} \widetilde{A}_{1}\right)\left(B_{1} \widetilde{A}_{1}\right)^{\dagger}=B_{1}$.

$\langle 121\rangle \mathscr{R}\left(A_{1} \widetilde{B}_{1}\right)=\mathscr{R}\left(A_{1}\right)$ and/or $\mathscr{R}\left(B_{1} \widetilde{A}_{1}\right)=\mathscr{R}\left(B_{1}\right)$.

$\langle 122\rangle\left(A_{1} \widetilde{B}_{1}\right)\left(A_{1} \widetilde{B}_{1}\right)^{\dagger}+\left(B_{1} \widetilde{A}_{1}\right)\left(B_{1} \widetilde{A}_{1}\right)^{\dagger}=A_{1}+B_{1}$.

$\langle 123\rangle A_{1}\left(A_{1}-B_{1}\right)^{\dagger} A_{1}=A_{1}$ and/or $B_{1}\left(B_{1}-A_{1}\right)^{\dagger} B_{1}=B_{1}$.

$\langle 124\rangle A_{1}\left(A_{1}-B_{1}\right)^{\dagger} A_{1}+B_{1}\left(B_{1}-A_{1}\right)^{\dagger} B_{1}=A_{1}+B_{1}$.

$\langle 125\rangle A_{1}+B_{1} \prec 2 I_{m}$.

$\langle 126\rangle\left[A_{1}, B_{1}, 0\right]\left[\begin{array}{ccc}A_{1} & 0 & I_{m} \\ 0 & B_{1} & I_{m} \\ I_{m} & I_{m} & 0\end{array}\right]^{\dagger}\left[\begin{array}{c}A_{1} \\ B_{1} \\ 0\end{array}\right]=A_{1}+B_{1}$.

$\langle 127\rangle\left[0,0, I_{m}\right]\left[\begin{array}{ccc}A_{1} & 0 & I_{m} \\ 0 & B_{1} & I_{m} \\ I_{m} & I_{m} & 0\end{array}\right]^{\dagger}\left[\begin{array}{c}0 \\ 0 \\ I_{m}\end{array}\right]=0$.

$\langle 128\rangle \lim _{k \rightarrow \infty}(A+B)^{k} / 2^{k}=0$.

$\langle 129\rangle \lim _{k \rightarrow \infty}(A B)^{k}=0$ and/or $\lim _{k \rightarrow \infty}(B A)^{k}=0$.

$\langle 130\rangle \lim _{k \rightarrow \infty}(A B A)^{k}=0$ and/or $\lim _{k \rightarrow \infty}(B A B)^{k}=0$.

$\langle 131\rangle \lim _{k \rightarrow \infty}(A B+B A)^{k} / 2^{k}=0$.

$\langle 132\rangle \lim _{k \rightarrow \infty}(A B A+B A B)^{k} / 2^{k}=0$. 
$\langle 133\rangle \lim _{k \rightarrow \infty}\left[A(A+B)^{\dagger} A\right]^{k}=A$ and/or $\lim _{k \rightarrow \infty}\left[B(A+B)^{\dagger} B\right]^{k}=B$.

$\langle 134\rangle \lim _{k \rightarrow \infty}\left(A_{1}+B_{1}\right)^{k} / 2^{k}=0$.

$\langle 135\rangle \lim _{k \rightarrow \infty}\left(2 I_{m}-A-B\right)^{\frac{1}{k}}=I_{m}$.

$\langle 136\rangle \lim _{k \rightarrow \infty}(A \widetilde{B} A)^{\frac{1}{k}}=A$ and/or $\lim _{k \rightarrow \infty}(B \widetilde{A} B)^{\frac{1}{k}}=B$.

$\langle 137\rangle \lim _{k \rightarrow \infty}\left(I_{m}-A B A\right)^{\frac{1}{k}}=I_{m}$ and/or $\lim _{k \rightarrow \infty}\left(I_{m}-B A B\right)^{\frac{1}{k}}=I_{m}$.

$\langle 138\rangle \lim _{k \rightarrow \infty}\left(2 I_{m}-A B A-B A B\right)^{\frac{1}{k}}=I_{m}$.

$\langle 139\rangle$ There exists an $A^{(1)}$ such that $A^{(1)} B=0$ and/or there exists a $B^{(1)}$ such that $B^{-} A=0$.

Proof. Eqs. (4.1) and (4.2) follow from $A-A_{2}=B-B_{3}$ in Corollary 3.6(b). The following identities can be verified from (3.3)-(3.7), Theorem 3.4(a), and the definition of the Moore-Penrose inverse

$$
\begin{aligned}
& A(A+B)^{\dagger} B=B(A+B)^{\dagger} A=U \operatorname{diag}\left(0_{2 k_{1}}, 2^{-1} I_{k_{3}}, 0_{k_{4}+k_{5}+k_{6}}\right) U^{*}, \\
& A(A+B)^{\dagger} A=U \operatorname{diag}\left(I_{k_{1}}, 0_{k_{2}}, 2^{-1} I_{k_{3}}, I_{k_{4}}, 0_{k_{5}+k_{6}}\right) U^{*}, \\
& B(A+B)^{\dagger} B=U \operatorname{diag}\left(\left[\begin{array}{ll}
C^{2} & C S \\
C S & S^{2}
\end{array}\right], 2^{-1} I_{k_{3}}, 0, I_{k_{5}}, 0_{k_{6}}\right) U^{*}, \\
& A(A+B)^{\dagger} A+B(A+B)^{\dagger} B=U \operatorname{diag}\left(\left[\begin{array}{cc}
I_{k_{1}}+C^{2} & C S \\
C S & S^{2}
\end{array}\right], I_{k_{3}+k_{4}+k_{5}}, 0_{k_{6}}\right) U^{*}, \\
& {\left[A(A+B)^{\dagger} A\right]^{\dagger}=U \operatorname{diag}\left(I_{k_{1}}, 0_{k_{2}}, 2 I_{k_{3}}, I_{k_{4}}, 0_{k_{5}+k_{6}}\right) U^{*},} \\
& {\left[B(A+B)^{\dagger} B\right]^{\dagger}=U \operatorname{diag}\left(\left[\begin{array}{cc}
C^{2} & C S \\
C S & S^{2}
\end{array}\right], 2 I_{k_{3}}, 0, I_{k_{5}}, 0_{k_{6}}\right) U^{*},} \\
& A(A-B)^{\dagger} A=U \operatorname{diag}\left(I_{k_{1}}, 0_{k_{2}+k_{3}}, I_{k_{4}}, 0_{k_{5}+k_{6}}\right) U^{*}, \\
& B(A-B)^{\dagger} B=-U \operatorname{diag}\left(\left[\begin{array}{ll}
C^{2} & C S \\
C S & S^{2}
\end{array}\right], 0_{k_{3}+k_{4}}, I_{k_{5}}, 0_{k_{6}}\right) U^{*}, \\
& A(A B+B A)^{\dagger} A=B A(A B+B A)^{\dagger} A B=U \operatorname{diag}\left(0_{2 k_{1}}, 2^{-1} I_{k_{3}}, 0_{k_{4}+k_{5}+k_{6}}\right) U^{*}, \\
& B(A B+B A)^{\dagger} B=A B(A B+B A)^{\dagger} B A=U \operatorname{diag}\left(0_{2 k_{1}}, 2^{-1} I_{k_{3}}, 0_{k_{4}+k_{5}+k_{6}}\right) U^{*}, \\
& A(B A B)^{\dagger} A=A B(A B)^{\dagger}=A_{1}, \quad B(A B A)^{\dagger} B=B A(B A)^{\dagger}=B_{1}, \\
& A(\widetilde{B} A \widetilde{B})^{\dagger} A=(A \widetilde{B})(A \widetilde{B})^{\dagger}=A_{2}, \quad \widetilde{B}(A \widetilde{B} A)^{\dagger} \widetilde{B}=(\widetilde{B} A)(\widetilde{B} A)^{\dagger}=B_{2}, \\
& \widetilde{A}(B \widetilde{A} B)^{\dagger} \widetilde{A}=\widetilde{A} B(\widetilde{A} B)^{\dagger}=A_{3}, \quad B(\widetilde{A} B \widetilde{A})^{\dagger} B=(B \widetilde{A})(B \widetilde{A})^{\dagger}=B_{3}, \\
& \widetilde{A}(\widetilde{B} \widetilde{A} \widetilde{B})^{\dagger} \widetilde{A}=(\widetilde{A} \widetilde{B})(\widetilde{A} \widetilde{B})^{\dagger}=A_{4}, \quad \widetilde{B}(\widetilde{A} \widetilde{B} \widetilde{A})^{\dagger} \widetilde{B}=(\widetilde{B} \widetilde{A})(\widetilde{B} \widetilde{A})^{\dagger}=B_{4} \text {, } \\
& A(A B A+B A B)^{\dagger} A=U \operatorname{diag}\left(C^{-2}, 0_{k_{2}}, 2^{-1} I_{k_{3}}, 0_{k_{4}+k_{5}+k_{6}}\right) U^{*} \text {, } \\
& B(A B A+B A B)^{\dagger} B=U \operatorname{diag}\left(\left[\begin{array}{cc}
I_{k_{1}} & C^{-1} S \\
C^{-1} S & C^{-2} S^{2}
\end{array}\right], 2^{-1} I_{k_{3}}, 0_{k_{4}+k_{5}+k_{6}}\right) U^{*}, \\
& A(A B A+B A B)^{\dagger} B=U \operatorname{diag}\left(0_{k_{1}+k_{2}}, 2^{-1} I_{k_{3}}, 0_{k_{4}+k_{5}+k_{6}}\right) U^{*}, \\
& A(A-B A B)^{\dagger} A=U \operatorname{diag}\left(I_{k_{1}}, 0_{k_{2}+k_{3}}, I_{k_{4}}, 0_{k_{5}+k_{6}}\right) U^{*}, \\
& B(B-A B A)^{\dagger} B=U \operatorname{diag}\left(\left[\begin{array}{ll}
C^{2} & C S \\
C S & S^{2}
\end{array}\right], 0_{k_{3}+k_{4}}, I_{k_{5}}, 0_{k_{6}}\right) U^{*} \text {. }
\end{aligned}
$$

Substituting (4.93)-(4.111) into (4.3)-(4.60), and compare the results obtained with the first equality in Theorem 3.4(e), we obtain the identities in (4.3)-(4.60).

In order to show (4.61) and (4.62), we need a result in [137]: for $0 \preccurlyeq A \in \mathbb{C}_{\mathrm{H}}^{m}$ and $B \in \mathbb{C}^{m \times n}$, the following expansion formula holds

$$
\left[\begin{array}{cc}
A & B \\
B^{*} & 0
\end{array}\right]^{\dagger}=\left[\begin{array}{cc}
\left(E_{B} A E_{B}\right)^{\dagger} & \left(B^{\dagger}\right)^{*}-\left(E_{B} A E_{B}\right)^{\dagger} A\left(B^{\dagger}\right)^{*} \\
B^{\dagger}-B^{\dagger} A\left(E_{B} A E_{B}\right)^{\dagger} & -B^{\dagger} A\left(B^{\dagger}\right)^{*}+B^{\dagger} A\left(E_{B} A E_{B}\right)^{\dagger} A\left(B^{\dagger}\right)^{*}
\end{array}\right] .
$$


Let $D=\left[\begin{array}{cc}A & 0 \\ 0 & B\end{array}\right]$ and $H=\left[\begin{array}{c}I_{m} \\ I_{m}\end{array}\right]$. Then we obtain from (4.112) that

$$
\left[\begin{array}{ccc}
A & 0 & I_{m} \\
0 & B & I_{m} \\
I_{m} & I_{m} & 0
\end{array}\right]^{\dagger}=\left[\begin{array}{cc}
S & * \\
* & T
\end{array}\right], H^{\dagger}=\frac{1}{2}\left[I_{m}, I_{m}\right], E_{H}=I_{2 m}-H H^{\dagger}=\frac{1}{2}\left[\begin{array}{cc}
I_{m} & -I_{m} \\
-I_{m} & I_{m}
\end{array}\right],
$$

where

$$
\begin{aligned}
S & =\left(E_{H} D E_{H}\right)^{\dagger}=\left[\begin{array}{cc}
\frac{1}{4}(A+B) & -\frac{1}{4}(A+B) \\
-\frac{1}{4}(A+B) & \frac{1}{4}(A+B)
\end{array}\right]^{\dagger}=\left[\begin{array}{cc}
(A+B)^{\dagger} & -(A+B)^{\dagger} \\
-(A+B)^{\dagger} & (A+B)^{\dagger}
\end{array}\right], \\
T & =-H^{\dagger} D\left(H^{\dagger}\right)^{*}+H^{\dagger} D\left(E_{H} D E_{H}\right)^{\dagger} D\left(H^{\dagger}\right)^{*} \\
& =-\frac{1}{4}(A+B)+\frac{1}{4} A(A+B)^{\dagger} A+\frac{1}{4} B(A+B)^{\dagger} B-\frac{1}{4} A(A+B)^{\dagger} B-\frac{1}{4} B(A+B)^{\dagger} A,
\end{aligned}
$$

so that

$$
\begin{aligned}
& \frac{1}{2} A+\frac{1}{2} B-\frac{1}{2}[A, B, 0]\left[\begin{array}{ccc}
A & 0 & I_{m} \\
0 & B & I_{m} \\
I_{m} & I_{m} & 0
\end{array}\right]^{\dagger}\left[\begin{array}{l}
A \\
B \\
0
\end{array}\right] \\
& =\frac{1}{2} A+\frac{1}{2} B-\frac{1}{2}[A, B] S\left[\begin{array}{l}
A \\
B
\end{array}\right] \\
& =\frac{1}{2} A+\frac{1}{2} B-\frac{1}{2} A(A+B)^{\dagger} A-\frac{1}{2} B(A+B)^{\dagger} B+\frac{1}{2} A(A+B)^{\dagger} B+\frac{1}{2} B(A+B)^{\dagger} A \\
& =P_{\mathcal{M} \cap \mathcal{N}}((\text { by (4.1) and (4.6)-(4.10)), }
\end{aligned}
$$

and

$$
\begin{aligned}
-2\left[0,0, I_{m}\right]\left[\begin{array}{ccc}
A & 0 & I_{m} \\
0 & B & I_{m} \\
I_{m} & I_{m} & 0
\end{array}\right]^{\dagger}\left[\begin{array}{c}
0 \\
0 \\
I_{m}
\end{array}\right]= & \frac{1}{2}(A+B)-\frac{1}{2} A(A+B)^{\dagger} A-\frac{1}{2} B(A+B)^{\dagger} B \\
& +\frac{1}{2} A(A+B)^{\dagger} B+\frac{1}{2} B(A+B)^{\dagger} A \\
= & P_{\mathcal{\mathcal { N } \cap N}}((\text { by }(4.1) \text { and }(4.6)-(4.10)),
\end{aligned}
$$

establishing the identities of (4.1), (4.61) and (4.62).

Similarly, we obtain from (4.113), (4.1), and (4.49)-(4.52) that

$$
\begin{aligned}
& \frac{1}{2} A B A+\frac{1}{2} B A B-\frac{1}{2}[A B A, B A B, 0]\left[\begin{array}{ccc}
A B A & 0 & I_{m} \\
0 & B A B & I_{m} \\
I_{m} & I_{m} & 0
\end{array}\right]^{\dagger}\left[\begin{array}{c}
A B A \\
B A B \\
0
\end{array}\right] \\
&=\frac{1}{2} A B A+\frac{1}{2} B A B-\frac{1}{2} A B A(A B A+B A B)^{\dagger} A B A-\frac{1}{2} B A B(A B A+B A B)^{\dagger} B A B \\
& \quad+\frac{1}{2} A B A(A B A+B A B)^{\dagger} B A B+\frac{1}{2} B A B(A B A+B A B)^{\dagger} A B A \\
&= P_{\mathcal{N} \cap \mathcal{N}}(\text { by (4.1) and (4.49)-(4.52)) } \\
&=-2\left[0,0, I_{m}\right]\left[\begin{array}{ccc}
A B A & 0 & I_{m} \\
0 & B A B & I_{m} \\
I_{m} & I_{m} & 0
\end{array}\right]^{\dagger}\left[\begin{array}{c}
0 \\
0 \\
I_{m}
\end{array}\right] \\
&= \frac{1}{2} A B A+\frac{1}{2} B A B-\frac{1}{2} A B A(A B A+B A B)^{\dagger} A B A-\frac{1}{2} B A B(A B A+B A B)^{\dagger} B A B \\
&+\frac{1}{2} A B A(A B A+B A B)^{\dagger} B A B+\frac{1}{2} B A B(A B A+A B A)^{\dagger} A B A
\end{aligned}
$$




$$
=P_{\mathcal{M} \cap \mathcal{N}}(\text { by (4.1) and (4.49)-(4.52)), }
$$

establishing the identities of (4.1), (4.63) and (4.64).

Replacing the two orthogonal projectors $A$ and $B$ with the two orthogonal projectors $A_{1}$ and $B_{1}$, and observing from Corollary 3.6(f) that $P_{\mathcal{M} \cap \mathcal{N}}=P_{\mathcal{S}_{1} \cap \mathcal{T}_{1}}$, we obtain (4.65)-(4.81) from (4.1)-(4.62).

Matrix decomposition identities of $(A+B)^{k},(A B)^{k},(A B A)^{k},(B A B)^{k},(A B+B A)^{k},(A B A+B A B)^{k},[A(A+$ $\left.B)^{\dagger} A\right]^{k},\left[B(A+B)^{\dagger} B\right]^{k},\left(A_{1}+B_{1}\right)^{k},(A \widetilde{B} A)^{1 / k},(B \widetilde{A} B)^{1 / k},\left(I_{m}-A B A\right)^{1 / k},\left(I_{m}-B A B\right)^{1 / k}$ and $\left(I_{m}-A B A-B A B\right)^{1 / k}$ can be established from Theorem 3.4(a), (4.94), and (4.95). Taking the limits of these matrix decomposition identities yields the equivalence of (4.1), (4.82)-(4.92).

Note that $\operatorname{dim}((\mathcal{N}+\mathcal{N})=r[A, B], \operatorname{dim}(\mathcal{N})=r(A)$ and $\operatorname{dim}(\mathcal{N})=r(B)$. Hence Statements $\langle 27\rangle$ and $\langle 35\rangle$ are equivalent. Applying the following well-known results in [103]

$$
r[X, Y]=r(X)+r\left(Y-X X^{\dagger} Y\right)=r(Y)+r\left(X-Y Y^{\dagger} X\right),
$$

and

$$
\begin{gathered}
r[X, Y]=r(X)+r(Y) \Leftrightarrow r\left(Y-X X^{\dagger} Y\right)=r(Y) \Leftrightarrow r\left(X-Y Y^{\dagger} X\right)=r(X) \\
\Leftrightarrow \mathscr{R}\left(Y^{*}-Y^{*} X X^{\dagger}\right)=\mathscr{R}\left(Y^{*}\right) \Leftrightarrow r\left(X^{*}-X^{*} Y Y^{\dagger}\right)=r\left(X^{*}\right)
\end{gathered}
$$

to $[A, B]$ lead to the equivalence of $\langle 42\rangle-\langle 44\rangle$. Setting (4.1)-(4.92) equal to zero yields the equivalences of $\langle 1\rangle$, and $\langle 45\rangle-\langle 138\rangle$.

The equivalence of $\langle 42\rangle$ and $\langle 139\rangle$ follows from the following known formulas

$$
\min _{A^{(1)}} r\left(A^{(1)} B\right)=\min _{B^{(1)}} r\left(B^{(1)} A\right)=r(A)+r(B)-r[A, B],
$$

see [133].

Some of the expressions in (4.1)-(4.92) were given in the literature; see e.g., [31, 49, 109, 116, 136]. Replacing $\mathcal{M}$ and $\mathcal{N}$ with $\mathcal{N}^{\perp}$ and $\mathcal{N}^{\perp}$ respectively in Theorem 4.1 will yield other three families of formulas for representing the orthogonal projectors onto the subspace intersections $\mathcal{M} \cap \mathcal{N}^{\perp}, \mathcal{M}^{\perp} \cap \mathcal{N}$, and $\mathcal{M}^{\perp} \cap \mathcal{N}^{\perp}$, respectively, as well as, a huge amount $\left(139^{4}\right)$ of identifying conditions for $\mathcal{M}$ and $\mathcal{N}$ to be in generic position. Here we select a group of such conditions as the representatives.

Theorem 4.2. Let $\mathcal{M}$ and $\mathcal{N}$ be two linear subspaces of $\mathbb{C}^{m}$ with $m$ even, $A=P_{\mathcal{M}}, B=P_{\mathcal{N}}, \mathcal{S}_{i}$, and $\mathcal{T}_{i}$ be as given in (3.18)-(3.21), $i=1,2,3$, 4. Then, the following statements are equivalent:

$\langle 1\rangle \mathcal{M}$ and $\mathcal{N}$ are in generic position.

〈2) $\mathcal{M}+\mathcal{N}=\mathcal{M}+\mathcal{N}^{\perp}=\mathcal{M}^{\perp}+\mathcal{N}=\mathcal{M}^{\perp}+\mathcal{N}^{\perp}=\mathbb{C}^{m}$.

$\langle 3\rangle \mathcal{M}=\mathcal{M} \cdot \mathcal{N}, \mathcal{N}=\mathcal{N} \bullet \mathcal{M}, \mathcal{M}^{\perp}=\mathcal{M}^{\perp} \bullet \mathcal{N}$ and $\mathcal{N}^{\perp}=\mathcal{N}^{\perp} \bullet \mathcal{M}$.

$\langle 4\rangle(\mathcal{M} \bullet \mathcal{N}) \oplus(\mathcal{N} \bullet \mathcal{M})=(\mathcal{M} \bullet \mathcal{N}) \oplus\left(\mathcal{N}^{\perp} \bullet \mathcal{M}\right)=\left(\mathcal{M}^{\perp} \bullet \mathcal{N}\right) \oplus(\mathcal{N} \bullet \mathcal{M})=\left(\mathcal{M}^{\perp} \bullet \mathcal{N}\right) \oplus\left(\mathcal{N}^{\perp} \bullet \mathcal{M}\right)=\mathbb{C}^{m}$.

$\langle 5\rangle \mathcal{S}_{i} \cap \mathcal{T}_{i}=\{0\}, i=1,2,3,4$.

$\langle 6\rangle r[A, B]=2 r(A)=2 r(B)=2 r(A B)=m$.

$\langle 7\rangle A+B, \widetilde{A}-B$, and $\widetilde{A}+\widetilde{B}$ are nonsingular.

$\langle 8\rangle A+B, \widetilde{A}-B$, and $2 I_{m}-A B-B A$ are nonsingular.

$\langle 9\rangle A+B, \widetilde{A}-B$, and $2 I_{m}-A B A-B A B$ are nonsingular.

$\langle 10\rangle$ Both $\widetilde{A}-B$ is nonsingular and $-I_{m} \prec \widetilde{A}-B \prec I_{m}$.

$\langle 11\rangle 0 \prec(\widetilde{A}-B)^{2} \prec I_{m}$.

$\langle 12\rangle-I_{m} \prec A-B \prec I_{m}$ and $A-B$ is nonsingular.

$\langle 13\rangle 0 \prec(A-B)^{2} \prec I_{m}$.

$\langle 14\rangle A B-B A$ is nonsingular.

$\langle 15\rangle A B A-B A B$ is nonsingular.

〈16) There exist a unitary matrix $U$ and a diagonal matrix $C$ with $0 \prec C \prec I_{m / 2}$ such that

$$
A=U\left[\begin{array}{cc}
I_{m / 2} & 0 \\
0 & 0
\end{array}\right] U^{*} \text { and } B=U\left[\begin{array}{cc}
C^{2} & C\left(I_{m / 2}-C^{2}\right)^{1 / 2} \\
C\left(I_{m / 2}-C^{2}\right)^{1 / 2} & I_{m / 2}-C^{2}
\end{array}\right] U^{*} .
$$


〈17〉 There exist a unitary matrix $U$ and a diagonal matrix $C$ with $0 \prec C \prec I_{m / 2}$ such that

$$
A+B=U \operatorname{diag}\left(I_{m / 2}-C, I_{m / 2}+C\right) U^{*} .
$$

$\langle 18\rangle$ There exist a unitary matrix $U$ and a diagonal matrix $C$ with $0 \prec S \prec I_{m / 2}$ such that

$$
A-B=U \operatorname{diag}(-S, S) U^{*} \text {. }
$$

$\langle 19\rangle$ There exist a unitary matrix $U$ and a diagonal matrix $C$ with $0 \prec C \prec I_{m / 2}$ such that

$$
A B=U\left[\begin{array}{cc}
C^{2} & C\left(I_{m / 2}-C^{2}\right)^{1 / 2} \\
0 & 0
\end{array}\right] U^{*} .
$$

$\langle 20\rangle$ There exist a unitary matrix $U$ and a diagonal matrix $C$ with $0 \prec C \prec I_{m / 2}$ such that

$$
A B+B A=U \operatorname{diag}\left(-C\left(I_{m / 2}-C\right), C\left(I_{m / 2}+C\right)\right) U^{*} .
$$

〈21) There exist a unitary matrix $U$ and a diagonal matrix $C$ with $0 \prec C \prec I_{m / 2}$ such that

$$
A B-B A=U\left[\begin{array}{cc}
0 & C\left(I_{m / 2}-C^{2}\right)^{1 / 2} \\
-C\left(I_{m / 2}-C^{2}\right)^{1 / 2} & 0
\end{array}\right] U^{*} .
$$

〈22〉 There exist a unitary matrix $U$ and a diagonal matrix $C$ with $0 \prec C \prec I_{m / 2}$ such that

$$
(A B-B A)^{2}=U \operatorname{diag}\left(-C^{2}\left(I_{m / 2}-C^{2}\right),-C^{2}\left(I_{m / 2}-C^{2}\right)\right) U^{*} .
$$

〈23〉 There exist a unitary matrix $U$ and a diagonal matrix $C$ with $0 \prec C \prec I_{m / 2}$ such that

$$
A B A=U\left(C^{2}, 0\right) U^{*}
$$

〈24〉 There exist a unitary matrix $U$ and a diagonal matrix $C$ with $0 \prec S \prec I_{m / 2}$ such that

$$
A-A B A=U \operatorname{diag}\left(S^{2}, 0\right) U^{*} .
$$

$\langle 25\rangle$ There exist a unitary matrix $U$ and a diagonal matrix $C$ with $0 \prec C \prec I_{m / 2}$ such that

$$
A B A+B A B=U \operatorname{diag}\left(C^{2}\left(I_{m / 2}-C\right), C^{2}\left(I_{m / 2}+C\right)\right) U^{*} .
$$

〈26〉 There exist a unitary matrix $U$ and a diagonal matrix $C$ with $0 \prec C \prec I_{m / 2}$ such that

$$
A B A-B A B=U \operatorname{diag}\left(-C^{2}\left(I_{m / 2}-C^{2}\right)^{1 / 2}, C^{2}\left(I_{m / 2}-C^{2}\right)^{1 / 2}\right) U^{*} .
$$

It is obvious that the parts of the matrices on the left-hand sides of the equalities in Theorem 3.4(a) are special cases of the following linear combination

$$
M=a_{0} I_{m}+a_{1} A+a_{2} B+a_{3} A B+a_{4} B A+a_{5} A B A+a_{6} B A B
$$

where $a_{0}, a_{1}, \ldots, a_{6} \in \mathbb{C}$. The special cases $a P_{\mathcal{M}}+b P_{\mathcal{N}}, a P_{\mathcal{M}}+b P_{\mathcal{N}}+c P_{\mathcal{M}} P_{\mathcal{N}}$, as well as, $a P_{\mathcal{M}}+b P_{\mathcal{N}}+c P_{\mathcal{M} \cap \mathcal{N}}$ were widely approached, in particular, people are interested in decomposing a matrix as given in (4.114), see e.g., [28, 29, 42, 65, 86, 92, 111, 125, 129, 132, 138, 141-143, 154]. By (3.3) and (3.4), the matrix $M$ in (4.114) can be decomposed as

$$
M=U \operatorname{diag}\left\{N, a I_{k_{3}},\left(a_{0}+a_{1}\right) I_{k_{4}},\left(a_{0}+a_{2}\right) I_{k_{5}}, a_{0} I_{k_{6}}\right\} U^{*},
$$

where $a=a_{0}+a_{1}+a_{2}+a_{3}+a_{4}+a_{5}+a_{6}$, and

$$
N=\left[\begin{array}{cc}
\left(a_{0}+a_{1}\right) I_{k_{1}}+\left(a_{2}+a_{3}+a_{4}+a_{5}\right) C^{2}+a_{6} C^{4} & \left(a_{2}+a_{3}\right) C S+a_{6} C^{3} S \\
\left(a_{2}+a_{4}\right) C S+a_{6} C^{3} S & a_{0} I_{k_{2}}+a_{2} S^{2}+a_{6} C^{2} S^{2}
\end{array}\right] .
$$

Hence, this fact shows that all behaviors of the $M$ can be characterized from its decomposition. We next give some consequences as illustration. 
(a) The determinant of $M$ is

$$
|M|=a_{0}^{k_{6}}\left(a_{0}+a_{1}\right)^{k_{4}}\left(a_{0}+a_{2}\right)^{k_{5}}\left(a_{0}+a_{1}+a_{2}+a_{3}+a_{4}+a_{5}+a_{6}\right)^{k_{3}}|N|,
$$

where

$$
\begin{aligned}
|N|=\mid & \left.\mid\left(a_{0}+a_{1}\right) I_{k_{1}}+\left(a_{2}+a_{3}+a_{4}+a_{5}\right) C^{2}+a_{6} C^{4}\right]\left[a_{0} I_{k_{2}}+a_{2} S^{2}+a_{6} C^{2} S^{2}\right] \\
& \left.-\left[\left(a_{2}+a_{3}\right) C S+a_{6} C^{3} S\right]\right]\left[\left(a_{2}+a_{4}\right) C S+a_{6} C^{3} S\right] \mid .
\end{aligned}
$$

(b) The rank of $M$ is

$$
r(M)=r(N)+k_{3} r(a)+k_{4} r\left(a_{0}+a_{1}\right)+k_{5} r\left(a_{0}+a_{2}\right)+k_{6} r\left(a_{0}\right) .
$$

(c) The Moore-Penrose inverse of $M$ is

$$
M^{\dagger}=U \operatorname{diag}\left\{N^{\dagger}, a^{\dagger} I_{k_{3}},\left(a_{0}+a_{1}\right)^{\dagger} I_{k_{4}},\left(a_{0}+a_{2}\right)^{\dagger} I_{k_{5}}, a_{0}^{\dagger} I_{k_{6}}\right\} U^{*} .
$$

In particular, $M$ is nonsingular if and only if $a_{0}\left(a_{0}+a_{1}\right)\left(a_{0}+a_{2}\right) a \neq 0$ and $|N| \neq 0$. In this case,

$$
M^{-1}=U \operatorname{diag}\left\{N^{-1}, a^{-1} I_{k_{3}},\left(a_{0}+a_{1}\right)^{-1} I_{k_{4}},\left(a_{0}+a_{2}\right)^{-1} I_{k_{5}}, a_{0}^{-1} I_{k_{6}}\right\} U^{*} .
$$

(d) Formulas for calculating the eigenvalues of $N$ can be derived. In particular, under the conditions $a_{0}, a_{1}, a_{2}, a_{5}, a_{6} \in \mathbb{R}$ and $a_{3} \in \mathbb{C}$, the linear combination

$$
M=a_{0} I_{m}+a_{1} A+a_{2} B+a_{3} A B+\overline{a_{3}} B A+a_{5} A B A+a_{6} B A B
$$

is Hermitian, and the partial inertia of $M$ is

$$
\begin{aligned}
& i_{ \pm}(M) \\
& =i_{ \pm}(N)+k_{3} i_{ \pm}\left(a_{0}+a_{1}+a_{2}+a_{3}+\overline{a_{3}}+a_{5}+a_{6}\right)+k_{4} i_{ \pm}\left(a_{0}+a_{1}\right)+k_{5} i_{ \pm}\left(a_{0}+a_{2}\right)+k_{6} i_{ \pm}\left(a_{0}\right),
\end{aligned}
$$

where

$$
N=\left[\begin{array}{cc}
\left(a_{0}+a_{1}\right) I_{k_{1}}+\left(a_{2}+a_{3}+\overline{a_{3}}+a_{5}\right) C^{2}+a_{6} C^{4} & \left(a_{2}+a_{3}\right) C S+a_{6} C^{3} S \\
\left(a_{2}+\overline{a_{3}}\right) C S+a_{6} C^{3} S & a_{0} I_{k_{2}}+a_{2} S^{2}+a_{6} C^{2} S^{2}
\end{array}\right] .
$$

Formulas for calculating the real eigenvalues of the matrix $N$ can be established.

In addition, more general linear combinations of the two orthogonal projectors $A, B$, and their products can be formulated, such as,

$$
a_{0} I_{m}+a_{1} A+a_{2} B+a_{3} A B+a_{4} B A+a_{5} A B A+a_{6} B A B+a_{7}(A B)^{2}+a_{8}(B A)^{2}+\cdots,
$$

see e.g., [44-46, 132]. It is not difficult to describe properties of such kind of matrix expressions and to establish fruitful results and facts on two orthogonal projectors and their algebraic operations.

\section{More on commutativity of two subspaces and two orthogonal projectors}

Commutativity of two linear subspaces, or equivalently, the commutative identity $A B=B A$ for the orthogonal projectors $A=P_{\mathcal{M}}$ and $B=P_{\mathcal{N}}$ is a quite special topic that attracts much attention in matrix theory, which has essential connections with many facts in matrix analysis and important applications in mathematics. There are many results on commutativity of two orthogonal projectors in the literature; see, e.g., [5, 7, 8, 10$12,14,15,18,19,21,33-35,76,85,93,112,122,123,132,135,140,147]$ and the relevant literature quoted there. In particular, it has closed link with reverse-order law problem $(M N)^{(i, \ldots, j)}=N^{(k, \ldots, l)} M^{(s, \ldots, t)}$ for generalized inverses of matrix product $M N$. To illustrate this link, we present a known fact. 
Lemma 5.1 $(89,140])$. Let $M \in \mathbb{C}^{m \times n}$ and $N \in \mathbb{C}^{n \times p}$. Then the following statements are equivalent:

\begin{tabular}{|c|c|c|c|}
\hline$\langle 1\rangle$ & $\left\{(M N)^{(1)}\right\} \supseteq\left\{N^{\dagger} M^{(1)}\right\}$. & $\langle 2\rangle$ & $\left\{(M N)^{(1)}\right\} \supseteq\left\{N^{\dagger} M^{(1,2)}\right\}$. \\
\hline$\langle 3\rangle$ & $\left\{(M N)^{(1)}\right\} \supseteq\left\{N^{\dagger} M^{(1,3)}\right\}$ & $\langle 4\rangle$ & $\left\{(M N)^{(1)}\right\} \supseteq\left\{N^{\dagger} M^{(1,4)}\right\}$ \\
\hline$\langle 5\rangle$ & $\left\{(M N)^{(1)}\right\} \supseteq\left\{N^{\dagger} M^{(1,2,3)}\right\}$. & $\langle 6\rangle$ & $\left\{(M N)^{(1)}\right\} \supseteq\left\{N^{\dagger} M^{(1,2,4)}\right\}$. \\
\hline$\langle 7\rangle$ & $\left\{(M N)^{(1)}\right\} \supseteq\left\{N^{\dagger} M^{(1,3,4)}\right\}$ & $\langle 8\rangle$ & $\left\{(M N)^{(1)}\right\} \supseteq\left\{N^{(1,3,4)} M^{(1)}\right\}$. \\
\hline$\langle 9\rangle$ & $\left\{(M N)^{(1)}\right\} \supseteq\left\{N^{(1,3,4)} M^{(1,2)}\right\}$. & $\langle 10\rangle$ & $\left\{(M N)^{(1)}\right\} \supseteq\left\{N^{(1,3,4)} M^{(1,3)}\right\}$. \\
\hline$\langle 11\rangle$ & $\left\{(M N)^{(1)}\right\} \supseteq\left\{N^{(1,3,4)} M^{(1,4)}\right\}$ & $\langle 12\rangle$ & $\left\{(M N)^{(1)}\right\} \supseteq\left\{N^{(1,3,4)} M^{(1,2,3)}\right\}$ \\
\hline$\langle 13\rangle$ & $\left\{(M N)^{(1)}\right\} \supseteq\left\{N^{(1,3,4)} M^{(1,2,4)}\right\}$. & $\langle 14\rangle$ & $\left\{(M N)^{(1)}\right\} \supseteq\left\{N^{(1,3,4)} M^{(1,3,4)}\right.$ \\
\hline$\langle 15\rangle$ & $\left\{(M N)^{(1)}\right\} \supseteq\left\{N^{(1,3,4)} M^{\dagger}\right\}$ & $\langle 16\rangle$ & $\left\{(M N)^{(1)}\right\} \supseteq\left\{N^{(1,2,4)} M^{(1,4)}\right\}$ \\
\hline$\langle 17\rangle$ & $\left\{(M N)^{(1)}\right\} \supseteq\left\{N^{(1,2,4)} M^{(1,2,4)}\right\}$. & $\langle 18\rangle$ & $\left\{(M N)^{(1)}\right\} \supseteq\left\{N^{(1,2,4)} M^{(1,3,4)}\right\}$ \\
\hline$\langle 19\rangle$ & $\left\{(M N)^{(1)}\right\} \supseteq\left\{N^{(1,2,4)} M^{\dagger}\right\}$ & $\langle 20\rangle$ & $\left\{(M N)^{(1)}\right\} \supseteq\left\{N^{(1,2,3)} M^{(1)}\right\}$ \\
\hline$\langle 21\rangle$ & $\left\{(M N)^{(1)}\right\} \supseteq\left\{N^{(1,2,3)} M^{(1,2)}\right\}$. & $\langle 22\rangle$ & $\left\{(M N)^{(1)}\right\} \supseteq\left\{N^{(1,2,3)} M^{(1,3)}\right\}$. \\
\hline$\langle 23\rangle$ & $\left\{(M N)^{(1)}\right\} \supseteq\left\{N^{(1,2,3)} M^{(1,4)}\right\}$ & $\langle 24\rangle$ & $\left\{(M N)^{(1)}\right\} \supseteq\left\{N^{(1,2,3)} M^{(1,2,3)}\right\}$ \\
\hline$\langle 25\rangle$ & $\left\{(M N)^{(1)}\right\} \supseteq\left\{N^{(1,2,3)} M^{(1,2,4)}\right\}$. & $\langle 26\rangle$ & $\left\{(M N)^{(1)}\right\} \supseteq\left\{N^{(1,2,3)} M^{(1,3,4}\right.$ \\
\hline$\langle 27\rangle$ & $\left\{(M N)^{(1)}\right\} \supseteq\left\{N^{(1,2,3)} M^{\dagger}\right\}$ & $\langle 28\rangle$ & $\left\{(M N)^{(1)}\right\} \supseteq\left\{N^{(1,4)} M^{(1,4)}\right\}$. \\
\hline$\langle 29\rangle$ & $\left\{(M N)^{(1)}\right\} \supseteq\left\{N^{(1,4)} M^{(1,2,4)}\right\}$. & $\langle 30\rangle$ & $\left\{(M N)^{(1)}\right\} \supseteq\left\{N^{(1,4)} M^{(1,3,4)}\right\}$. \\
\hline$\langle 31\rangle$ & $\left\{(M N)^{(1)}\right\} \supseteq\left\{N^{(1,4)} M^{\dagger}\right\}$ & $\langle 32\rangle$ & $\left\{(M N)^{(1)}\right\} \supseteq\left\{N^{(1,3)} M^{(1)}\right\}$. \\
\hline$\langle 33\rangle$ & $\left\{(M N)^{(1)}\right\} \supseteq\left\{N^{(1,3)} M^{(1,2)}\right\}$. & $\langle 34\rangle$ & $\left\{(M N)^{(1)}\right\} \supseteq\left\{N^{(1,3)} M^{(1,3)}\right\}$ \\
\hline$\langle 35\rangle$ & $\left\{(M N)^{(1)}\right\} \supseteq\left\{N^{(1,3)} M^{(1,4)}\right\}$ & $\langle 36\rangle$ & $\left\{(M N)^{(1)}\right\} \supseteq\left\{N^{(1,3)} M^{(1,2,3)}\right\}$. \\
\hline$\langle 37\rangle$ & $\left\{(M N)^{(1)}\right\} \supseteq\left\{N^{(1,3)} M^{(1,2,4)}\right\}$. & $\langle 38\rangle$ & $\left\{(M N)^{(1)}\right\} \supseteq\left\{N^{(1,3)} M^{(1,3,4)}\right\}$. \\
\hline$\langle 39\rangle$ & $\left\{(M N)^{(1)}\right\} \supseteq\left\{N^{(1,3)} M^{\dagger}\right\}$ & $\langle 40\rangle$ & $\left\{(M N)^{(1)}\right\} \supseteq\left\{N^{(1,2)} M^{(1,4)}\right\}$. \\
\hline$\langle 41\rangle$ & $\left\{(M N)^{(1)}\right\} \supseteq\left\{N^{(1,2)} M^{(1,2,4)}\right\}$ & $\langle 42\rangle$ & $\left\{(M N)^{(1)}\right\} \supseteq\left\{N^{(1,2)} M^{(1,3,4)}\right\}$ \\
\hline$\langle 43\rangle$ & $\left\{(M N)^{(1)}\right\} \supseteq\left\{N^{(1,2)} M^{\dagger}\right\}$ & $\langle 44\rangle$ & $\left\{(M N)^{(1)}\right\} \supseteq\left\{N^{(1)} M^{(1,4)}\right\}$ \\
\hline$\langle 45\rangle$ & $\left\{(M N)^{(1)}\right\} \supseteq\left\{N^{(1)} M^{(1,2,4)}\right\}$. & $\langle 46\rangle$ & $\left\{(M N)^{(1)}\right\} \supseteq\left\{N^{(1)} M^{(1,3,4)}\right\}$. \\
\hline$\langle 47\rangle$ & $\left\{(M N)^{(1)}\right\} \supseteq\left\{N^{(1)} M^{\dagger}\right\}$ & $\langle 48\rangle$ & $\left\{(M N)^{(1)}\right\} \ni N^{\dagger} M^{\dagger}$ \\
\hline$\langle 49$ & $M N N^{\dagger} M^{\dagger} M N=M N$ & $\langle 50\rangle$ & $\left(M^{\dagger} M\right)\left(N N^{\dagger}\right)=\left(N N^{\dagger}\right)\left(M^{\dagger} M\right)$ \\
\hline
\end{tabular}

Lemma 5.1 shows that the matrix set inclusions and matrix equality problem in $\langle 1\rangle-\langle 49\rangle$ are equivalent to the commutativity of the two orthogonal projectors $M^{\dagger} M$ and $N N^{\dagger}$. Based on the results in the previous sections, we obtain by observation the following equivalent statements for the commutative identity $A B=B A$ to hold, some of which were established in the references mentioned above.

Theorem 5.2. Let $\mathcal{N}$ and $\mathcal{N}$ be two linear subspaces in $\mathbb{C}^{m}, A, B, \widetilde{A}$, and $\widetilde{B}$ be as given in (3.2), $s$ be an integer, and $k_{3}, k_{4}, k_{5}, k_{6}$ be as given in (3.8) and (3.9), respectively. Then, the following statements are equivalent:

<1) $\mathcal{M}$ and $\mathcal{N}$ commute.

$\langle 2\rangle-\langle 24\rangle$ in Corollary 2.14.

25〉 Some/all of the four facts: $A B=B A, A \widetilde{B}=\widetilde{B} A, \widetilde{A} B=B \widetilde{A}, \widetilde{A} \widetilde{B}=\widetilde{B} \widetilde{A}$.

〈26) Some/all of the four facts: $A B A=B A B, A \widetilde{B} A=\widetilde{B} A \widetilde{B}, \widetilde{A} B \widetilde{A}=B \widetilde{A} B, \widetilde{A} \widetilde{B} \widetilde{A}=\widetilde{B} \widetilde{A} \widetilde{B}$.

227 There exists a unitary matrix $U$ such that

$$
A=U \operatorname{diag}\left(I_{k_{3}+k_{4}}, 0_{k_{5}+k_{6}}\right) U^{*} \text { and } B=U \operatorname{diag}\left(I_{k_{3}}, 0_{k_{4}}, I_{k_{5}}, 0_{k_{6}}\right) U^{*} \text {. }
$$

$\langle 28\rangle$ There exists a unitary matrix $U$ such that

$$
\alpha A+\beta B=U \operatorname{diag}\left((\alpha+\beta) I_{k_{3}}, \alpha I_{k_{4}}, \beta I_{k_{5}}, 0_{k_{6}}\right) U^{*}
$$

for some/all two nonzero real numbers $\alpha$ and $\beta$.

$\langle 29\rangle$ There exists a unitary matrix $U$ such that

$$
A B=U \operatorname{diag}\left(I_{k_{3}}, 0_{m-k_{3}}\right) U^{*} \text { and } / \text { or } B A=U \operatorname{diag}\left(I_{k_{3}}, 0_{m-k_{3}}\right) U^{*},
$$

namely, $A B$ and/or $B A$ is an orthogonal projector. 
$\langle 30\rangle$ There exists a unitary matrix $U$ such that

$$
\alpha A B+\beta B A=U \operatorname{diag}\left((\alpha+\beta) I_{k_{3}}, 0_{m-k_{3}}\right) U^{*}
$$

for some/all two nonzero real numbers $\alpha$ and $\beta$.

$\langle 31\rangle$ There exists a unitary matrix $U$ such that

$$
A B A=U \operatorname{diag}\left(I_{k_{3}}, 0_{m-k_{3}}\right) U^{*} \text { and } / \text { or } B A B=U \operatorname{diag}\left(I_{k_{3}}, 0_{m-k_{3}}\right) U^{*},
$$

namely, $A B A$ and/or $B A B$ is an orthogonal projector.

$\langle 32\rangle$ There exists a unitary matrix $U$ such that

$$
\alpha A B A+\beta B A B=U \operatorname{diag}\left((\alpha+\beta) I_{k_{3}}, 0_{m-k_{3}}\right) U^{*}
$$

for some/all two nonzero real numbers $\alpha$ and $\beta$.

$\langle 33\rangle$ There exists a unitary matrix $U$ such that

$$
\alpha A+\beta B A B=U \operatorname{diag}\left((\alpha+\beta) I_{k_{3}}, \alpha I_{k_{4}}, 0_{k_{5}+k_{6}}\right) U^{*}
$$

and/or

$$
\alpha B+\beta A B A=U \operatorname{diag}\left((\alpha+\beta) I_{k_{3}}, 0_{k_{4}}, \alpha I_{k_{5}}, 0_{k_{6}}\right) U^{*}
$$

for some/all two nonzero real numbers $\alpha$ and $\beta$.

〈34 There exists a unitary matrix $U$ such that

$$
\alpha(A B)^{\dagger}+\beta(B A)^{\dagger}=U \operatorname{diag}\left((\alpha+\beta) I_{k_{3}}, 0_{m-k_{3}}\right) U^{*}
$$

for some/all two nonzero real numbers $\alpha$ and $\beta$.

$\langle 35\rangle$ There exists a unitary matrix $U$ such that

$$
\alpha(A B A)^{\dagger}+\beta(B A B)^{\dagger}=U \operatorname{diag}\left((\alpha+\beta) I_{k_{3}}, 0_{m-k_{3}}\right) U^{*}
$$

for some/all two nonzero real numbers $\alpha$ and $\beta$.

$\langle 36\rangle(A+B)^{2} \succcurlyeq(A-B)^{2}$, i.e., $A B+B A \succcurlyeq 0$.

$\langle 37\rangle(A-B)^{2} \succcurlyeq A-B$ and/or $(B-A)^{2} \succcurlyeq B-A$.

$\langle 38\rangle(A-B)^{2} \in \mathbb{C}_{\mathrm{OP}}^{m}$.

$\langle 39\rangle(A-B)^{2^{s}+1}=A-B$ for some/all $s \geqslant 1$.

$\langle 40\rangle(A-B)^{\dagger}=A-B$.

$\langle 41\rangle A(A-B)^{\dagger}=(A-B)^{\dagger} A$ and $/$ or $B(A-B)^{\dagger}=(A-B)^{\dagger} B$.

$\langle 42\rangle A(A-B)^{\dagger}=(A-B)^{\dagger} A$ and/or $B(A-B)^{\dagger}=(A-B)^{\dagger} B$.

$\langle 43\rangle(A+B)(A-B)^{\dagger}=(A-B)^{\dagger}(A+B)$.

〈44〉 $A(A+B)^{\dagger} A+B(A+B)^{\dagger} B$ is an orthogonal projector.

$\langle 45\rangle A(A+B)^{\dagger}=(A+B)^{\dagger} A$ and/or $B(A+B)^{\dagger}=(A+B)^{\dagger} B$.

$\langle 46\rangle\left(I_{m}-A-B\right)^{2} \succcurlyeq I_{m}-A-B$ and/or $\left(A+B-I_{m}\right)^{2} \succcurlyeq A+B-I_{m}$.

$\langle 47\rangle(I-A-B)^{2}$ is an orthogonal projector.

$\langle 48\rangle\left(I_{m}-A-B\right)^{2^{s}+1}=I_{m}-A-B$ for some/all $s \geqslant 1$.

$\langle 49\rangle\left(I_{m}-A-B\right)^{\dagger}=I_{m}-A-B$.

$\langle 50\rangle(A-B)\left(I_{m}-A-B\right)^{\dagger}=\left(I_{m}-A-B\right)^{\dagger}(A-B)$.

$\langle 51\rangle(A+B)(A+B)^{\dagger}=A+B-A B$ and $/$ or $(A+B)(A+B)^{\dagger}=A+B-B A$.

$\langle 52\rangle\left[(A+B)(A+B)^{\dagger}-A\right]\left[(A+B)(A+B)^{\dagger}-B\right]=0$ and $/$ or $\left[(A+B)(A+B)^{\dagger}-B\right]\left[(A+B)(A+B)^{\dagger}-A\right]=0$.

$\langle 53\rangle(A+B)(A+B)^{\dagger}=A+\widetilde{A} B \widetilde{A}$ and/or $(A+B)(A+B)^{\dagger}=B+\widetilde{B} A \widetilde{B}$.

〈54〉 Some/all of the four facts: $A+B=(A-B)(A-B)^{\dagger}+2(A B)(A B)^{\dagger}, A+B=(A-B)(A-B)^{\dagger}+2(B A)(B A)^{\dagger}$, $A+B=(A-B)(A-B)^{\dagger}+2(A B)^{\dagger}(A B), A+B=(A-B)(A-B)^{\dagger}+2(B A)^{\dagger}(B A)$.

〈55〉 Some/all of the four facts: $A \succcurlyeq(B A)(B A)^{\dagger}, A \succcurlyeq(A B)^{\dagger}(A B), B \succcurlyeq(A B)(A B)^{\dagger}, B \succcurlyeq(B A)^{\dagger}(B A)$.

$\langle 56\rangle A+B \succcurlyeq(A B)(A B)^{\dagger}+(B A)(B A)^{\dagger}$ and/or $A+B \succcurlyeq(A B)^{\dagger}(A B)+(B A)^{\dagger}(B A)$. 
〈57〉 Some/all of the four facts: $A \succcurlyeq(\widetilde{B} A)(\widetilde{B} A)^{\dagger}, A \succcurlyeq(A \widetilde{B})^{\dagger}(A \widetilde{B}), B \succcurlyeq(\widetilde{A} B)(\widetilde{A} B)^{\dagger}, B \succcurlyeq(B \widetilde{A})^{\dagger}(B \widetilde{A})$.

$\langle 58\rangle A+B \succcurlyeq(\widetilde{A} B)(\widetilde{A} B)^{\dagger}+(\widetilde{B} A)(\widetilde{B} A)^{\dagger}$ and $/$ or $A+B \succcurlyeq(B \widetilde{A})^{\dagger}(B \widetilde{A})+(A \widetilde{B})^{\dagger}(A \widetilde{B})$.

〈59) $2 A(A B+B A)^{\dagger} B=A B$ and/or $2 B(A B+B A)^{\dagger} A=B A$.

$\langle 60\rangle 2 A(A B+B A)^{\dagger} B+2 B(A B+B A)^{\dagger} A=A B+B A$.

$\langle 61\rangle(A B)^{s} \in \mathbb{C}_{\mathrm{OP}}^{m}$ for some/all $s \geqslant 2$.

$\langle 62\rangle(A B)^{s}=A B$ for some/all $s \geqslant 2$.

$\langle 63\rangle(A B)^{S}=(B A)^{s}$ for some/all $s \geqslant 1$.

$\langle 64\rangle(A B)^{s}=B A$ for some/all $s \geqslant 2$.

$\langle 65\rangle(A B)^{s}+(B A)^{s}=A B+B A$ for some/all $s \geqslant 2$.

$\langle 66\rangle(A B A)^{s} \in \mathbb{C}_{\mathrm{OP}}^{m}$ and/or $(B A B)^{s} \in \mathbb{C}_{\mathrm{OP}}^{m}$ for some/all $s \geqslant 2$.

$\langle 67\rangle(A B A)^{s}=A B A$ and/or $(B A B)^{s}=B A B$ for some/all $s \geqslant 2$.

$\langle 68\rangle(A B A)^{s}=B A B$ and/or $(B A B)^{S}=A B A$ for some/all $s \geqslant 2$.

$\langle 69\rangle(A B A)^{S}+(B A B)^{S}=A B A+B A B$ for some/all $s \geqslant 2$.

$\langle 70\rangle(A B A)^{s}=A B$ and/or $(B A B)^{s}=B A$ for some/all $s \geqslant 1$.

$\langle 71\rangle(A B A)^{s}+(B A B)^{s}=A B+B A$ for some/all $s \geqslant 1$.

$\langle 72\rangle\left(I_{m}-A B\right)^{s}=I_{m}-A B$ for some/all $s \geqslant 2$.

$\langle 73\rangle\left(I_{m}-A B\right)^{s}=I_{m}-B A$ for some/all $s \geqslant 2$.

$\langle 74\rangle\left(I_{m}-A B\right)^{s}=\left(I_{m}-B A\right)^{s}$ for some/all $s \geqslant 1$.

$\langle 75\rangle(A+B-A B)^{s}=A+B-A B$ for some/all $s \geqslant 2$.

$\langle 76\rangle(A+B-A B)^{\dagger}=A+B-A B$.

$\langle 77\rangle A+B-A B \in \mathbb{C}_{\mathrm{OP}}^{m}$.

$\langle 78\rangle(A B+B A)^{2 s}=2^{2 s-1}(A B+B A)$ for some/all $s \geqslant 1$.

$\langle 79\rangle\left(I_{m}-A B-B A\right)^{2}=I_{m}$.

$\langle 80\rangle I_{m}+A B \in\left\{\left(I_{m}-A B\right)^{-}\right\}$and/or $I_{m}+B A \in\left\{\left(I_{m}-B A\right)^{-}\right\}$.

$\langle 81\rangle\left(I_{m}-A B A\right)^{s}=I_{m}-A B A$ and/or $\left(I_{m}-B A B\right)^{s}=I_{m}-B A B$ for some/all $s \geqslant 2$.

$\langle 82\rangle\left(I_{m}-A B A\right)^{s}+\left(I_{m}-B A B\right)^{s}=2 I_{m}-A B A-B A B$ for some/all $s \geqslant 2$.

$\langle 83\rangle(A B A+B A B)^{s}=2^{s-1}(A B A+B A B)$ for some/all $s \geqslant 2$.

$\langle 84\rangle\left(I_{m}-A B A-B A B\right)^{2}=I_{m}$.

$\langle 85\rangle(A-B A B)^{s}=A-B A B$ and/or $(B-A B A)^{s}=B-A B A$ for some/all $s \geqslant 2$.

$\langle 86\rangle(A-B A B)^{s}+(B-A B A)^{s}=A+B-A B A-B A B$ for some/all $s \geqslant 2$.

$\langle 87\rangle A-B A B \succcurlyeq 0$ and/or $B-A B A \succcurlyeq 0$.

$\langle 88\rangle A+B-A B A-B A B \succcurlyeq 0$.

$\langle 89\rangle A-B A B \in \mathbb{C}_{\mathrm{OP}}^{m}$ and/or $B-A B A \in \mathbb{C}_{\mathrm{OP}}^{m}$.

$\langle 90\rangle(A+B-A B A-B A B) / 2 \in \mathbb{C}_{\mathrm{OP}}^{m}$.

$\langle 91\rangle A B A+\widetilde{A} B \widetilde{A} \in \mathbb{C}_{\mathrm{OP}}^{m}$ and/or $B A B+\widetilde{B} A \widetilde{B} \in \mathbb{C}_{\mathrm{OP}}^{m}$.

$\langle 92\rangle A B A+\widetilde{A} B \widetilde{A}=B$ and $/$ or $B A B+\widetilde{B} A \widetilde{B}=A$.

$\langle 93\rangle A B \widetilde{A}+\widetilde{A} B A=0$ and/or $B A \widetilde{B}+\widetilde{B} A B=0$, i.e., $A B+B A=2 A B A$ and/or $A B+B A=2 B A B$.

$\langle 94\rangle(A B)^{\dagger}=B A$ and $/$ or $(B A)^{\dagger}=A B$.

$\langle 95\rangle(\widetilde{A} B)^{\dagger}=B \widetilde{A}$ and $/$ or $(B \widetilde{A})^{\dagger}=\widetilde{A} B$.

$\langle 96\rangle(A \widetilde{B})^{\dagger}=\widetilde{B} A$ and/or $(\widetilde{B} A)^{\dagger}=A \widetilde{B}$.

$\langle 97\rangle(\widetilde{A} \widetilde{B})^{\dagger}=\widetilde{B} \widetilde{A}$ and/or $(\widetilde{B} \widetilde{A})^{\dagger}=\widetilde{A} \widetilde{B}$.

$\langle 98\rangle(A B)^{\dagger}=A B$ and/or $(B A)^{\dagger}=B A$.

$\langle 99\rangle(\widetilde{A} B)^{\dagger}=\widetilde{A} B$ and/or $(B \widetilde{A})^{\dagger}=B \widetilde{A}$.

$\langle 100\rangle(A \widetilde{B})^{\dagger}=A \widetilde{B}$ and $/$ or $(\widetilde{B} A)^{\dagger}=\widetilde{B} A$.

$\langle 101\rangle(\widetilde{A} \widetilde{B})^{\dagger}=\widetilde{A} \widetilde{B}$ and $/$ or $(\widetilde{B} \widetilde{A})^{\dagger}=\widetilde{B} \widetilde{A}$.

$\langle 102\rangle$ Some/all of the four facts: $(A B)^{\dagger}+(B A)^{\dagger}=A B+B A,(\widetilde{A} B)^{\dagger}+(B \widetilde{A})^{\dagger}=\widetilde{A} B+B \widetilde{A},(A \widetilde{B})^{\dagger}+(\widetilde{B} A)^{\dagger}=A \widetilde{B}+\widetilde{B} A$, $(\widetilde{A} \widetilde{B})^{\dagger}+(\widetilde{B} \widetilde{A})^{\dagger}=\widetilde{A} \widetilde{B}+\widetilde{B} \widetilde{A}$.

$\langle 103\rangle$ Some/all of the eight facts: $\left[(A B)^{2}\right]^{\dagger}=\left[(A B)^{\dagger}\right]^{2},\left[(B A)^{2}\right]^{\dagger}=\left[(B A)^{\dagger}\right]^{2},\left[(\widetilde{A} B)^{2}\right]^{\dagger}=\left[(\widetilde{A} B)^{\dagger}\right]^{2},\left[(B \widetilde{A})^{2}\right]^{\dagger}=$ $\left[(B \widetilde{A})^{\dagger}\right]^{2},\left[(A \widetilde{B})^{2}\right]^{\dagger}=\left[(A \widetilde{B})^{\dagger}\right]^{2},\left[(\widetilde{B} A)^{2}\right]^{\dagger}=\left[(\widetilde{B} A)^{\dagger}\right]^{2},\left[(\widetilde{A} \widetilde{B})^{2}\right]^{\dagger}=\left[(\widetilde{A} \widetilde{B})^{\dagger}\right]^{2},\left[(\widetilde{B} \widetilde{A})^{2}\right]^{\dagger}=\left[(\widetilde{B} \widetilde{A})^{\dagger}\right]^{2}$.

$\left\langle\right.$ 104〉 Some/all of the four facts: $\left[(A B)^{\dagger}+(B A)^{\dagger}\right] / 2 \in \mathbb{C}_{\mathrm{OP}}^{m},\left[(\widetilde{A} B)^{\dagger}+(B \widetilde{A})^{\dagger}\right] / 2 \in \mathbb{C}_{\mathrm{OP}}^{m},\left[(A \widetilde{B})^{\dagger}+(\widetilde{B} A)^{\dagger}\right] / 2 \in \mathbb{C}_{\mathrm{OP}}^{m}$, $\left[(\widetilde{A} \widetilde{B})^{\dagger}+(\widetilde{B} \widetilde{A})^{\dagger}\right] / 2 \in \mathbb{C}_{\mathrm{OP}}^{m}$ 
$\langle 105\rangle(A B)(A B)^{\dagger}=A B A$ and $/$ or $B A(B A)^{\dagger}=B A B$.

$\langle 106\rangle(A B)(A B)^{\dagger}+(B A)(B A)^{\dagger}=A B A+B A B$.

$\langle 107\rangle(A B)(B A)^{\dagger}=(B A)(A B)^{\dagger}$.

$\langle 108\rangle\left[(A B)(A B)^{\dagger}\right]\left[(B A)(B A)^{\dagger}\right]=\left[(B A)(B A)^{\dagger}\right]\left[(A B)(A B)^{\dagger}\right]$.

$\langle 109\rangle A(\widetilde{A} \widetilde{B})^{\dagger} B=0$ and/or $B(\widetilde{B} \widetilde{A})^{\dagger} A=0$.

$\langle 110\rangle A(\widetilde{A} \widetilde{B})^{\dagger} B+B(\widetilde{B} \widetilde{A})^{\dagger} A=0$.

$\langle 111\rangle(A B)^{\#} \in \mathbb{C}_{\mathrm{OP}}^{m}$ and/or $(B A)^{\#} \in \mathbb{C}_{\mathrm{OP}}^{m}$.

$\langle 112\rangle\left[(A B)^{\#}+(B A)^{\#}\right] / 2 \in \mathbb{C}_{\mathrm{OP}}^{m}$.

$\langle 113\rangle(A B)^{\#}=(A B)^{\dagger}$ and/or $(B A)^{\#}=(B A)^{\dagger}$.

$\langle 114\rangle(A B)^{\#}+(B A)^{\#}=(A B)^{\dagger}+(B A)^{\dagger}$.

$\langle 115\rangle(A B)(A B)^{\#}=(A B)(A B)^{\dagger}$ and/or $(B A)(B A)^{\#}=(B A)(B A)^{\dagger}$.

$\langle 116\rangle(A B)(A B)^{\#}+(B A)(B A)^{\#}=(A B)(A B)^{\dagger}+(B A)(B A)^{\dagger}$.

$\langle 117\rangle(A B)^{\#}=A B$ and $/$ or $(B A)^{\#}=B A$.

$\langle 118\rangle(A B)^{\#}+(B A)^{\#}=A B+B A$.

$\langle 119\rangle(A B A)^{\dagger}=A B A$ and $/$ or $(B A B)^{\dagger}=B A B$.

$\langle 120\rangle(A B A)^{\dagger}+(B A B)^{\dagger}=A B A+B A B$.

$\langle 121\rangle\left(I_{m}-A B\right)^{\dagger}=I_{m}-A B$ and/or $\left(I_{m}-B A\right)^{\dagger}=I_{m}-B A$.

$\langle 122\rangle\left(I_{m}-A B\right)^{\dagger}=I_{m}-B A$ and/or $\left(I_{m}-B A\right)^{\dagger}=I_{m}-A B$.

$\langle 123\rangle\left(I_{m}-A B\right)^{\dagger}+\left(I_{m}-B A\right)^{\dagger}=2 I_{m}-A B-B A$.

$\langle 124\rangle\left[\left(I_{m}-A B\right)^{2}\right]^{\dagger}=\left[\left(I_{m}-A B\right)^{\dagger}\right]^{2}$ and/or $\left[\left(I_{m}-B A\right)^{2}\right]^{\dagger}=\left[\left(I_{m}-B A\right)^{\dagger}\right]^{2}$.

$\langle 125\rangle A\left(I_{m}-B A\right)^{\dagger} B=0$ and/or $B\left(I_{m}-A B\right)^{\dagger} A=0$.

$\langle 126\rangle A\left(I_{m}-B A\right)^{\dagger} B+B\left(I_{m}-A B\right)^{\dagger} A=0$.

$\langle 127\rangle\left[\left(I_{m}-A B\right)^{\dagger}+\left(I_{m}-B A\right)^{\dagger}\right] / 2 \in \mathbb{C}_{\mathrm{OP}}^{m}$.

$\langle 128\rangle\left(2 I_{m}-A-B\right)\left(2 I_{m}-A-B\right)^{\dagger}=I_{m}-A B$ and/or $\left(2 I_{m}-A-B\right)\left(2 I_{m}-A-B\right)^{\dagger}=I_{m}-B A$.

$\langle 129\rangle\left(2 I_{m}-A B-B A\right)\left(2 I_{m}-A B-B A\right)^{\dagger}=I_{m}-A B$ and/or $\left(2 I_{m}-A B-B A\right)\left(2 I_{m}-A B-B A\right)^{\dagger}=I_{m}-B A$.

$\langle 130\rangle\left(I_{m}-A B A\right)^{\dagger}=I_{m}-A B A$ and/or $\left(I_{m}-B A B\right)^{\dagger}=I_{m}-B A B$.

$\langle 131\rangle\left(I_{m}-A B A\right)^{\dagger}+\left(I_{m}-B A B\right)^{\dagger}=2 I_{m}-A B A-B A B$.

$\langle 132\rangle\left[(A B A)^{\dagger}+(B A B)^{\dagger}\right] / 2 \in \mathbb{C}_{\mathrm{OP}}^{m}$.

$\langle 133\rangle A\left(I_{m}-A B A\right)^{\dagger} B=0$ and/or $A\left(I_{m}-B A B\right)^{\dagger} B=0$.

$\langle 134\rangle A\left(I_{m}-A B A\right)^{\dagger} B+A\left(I_{m}-B A B\right)^{\dagger} B=0$.

$\langle 135\rangle\left(2 I_{m}-A B A-B A B\right)\left(2 I_{m}-A B A-B A B\right)^{\dagger}=I_{m}-A B A$ and $/$ or $\left(2 I_{m}-A B A-B A B\right)\left(2 I_{m}-A B A-B A B\right)^{\dagger}=$ $I_{m}-B A B$.

$\langle 136\rangle \operatorname{Trace}\left[(A B)^{s}\right]=\operatorname{Trace}(A B)$ for some/all $s \geqslant 2$.

$\langle 137\rangle$ Some/all of the four facts: $r(A B)=\operatorname{Trace}(A B), r(\widetilde{A} B)=\operatorname{Trace}(\widetilde{A} B), r(A \widetilde{B})=\operatorname{Trace}(A \widetilde{B}), r(\widetilde{A} \widetilde{B})=$ Trace $(\widetilde{A} \widetilde{B})$.

$\langle 138\rangle \operatorname{Trace}\left[(A-B)^{2}\right]=r(A)+r(B)-2 r(A B)$.

$\langle 139\rangle \operatorname{Trace}\left[(A-B)^{2}\right]=\operatorname{Trace}(A)+\operatorname{Trace}(B)-2 \mathrm{r}(A B)$.

$\langle 140\rangle \operatorname{Trace}\left[(A-B)^{2 s}\right]=$ Trace $\left[(A-B)^{2}\right]$ for some/all $s \geqslant 2$.

$\langle 141\rangle \operatorname{Trace}\left[\left(I_{m}-A B\right)^{s}\right]=\operatorname{Trace}\left(I_{m}-A B\right)$ for some/all $s \geqslant 2$.

$\langle 142\rangle \operatorname{Trace}\left[\left(I_{m}-A B A\right)^{s}\right]=\operatorname{Trace}\left(I_{m}-A B A\right)$ and/or Trace $\left[\left(I_{m}-B A B\right)^{s}\right]=\operatorname{Trace}\left(I_{m}-B A B\right)$ for some/all $s \geqslant 2$.

$\langle 143\rangle$ Some/all of the four facts: $\operatorname{dim}(\mathcal{M} \cap \mathcal{N})=r(A B), \operatorname{dim}\left(\mathcal{M}^{\perp} \cap \mathcal{N}\right)=r(\widetilde{A} B), \operatorname{dim}\left(\mathcal{M} \cap \mathcal{N}^{\perp}\right)=r(A \widetilde{B}), \operatorname{dim}\left(\mathcal{M}^{\perp} \cap\right.$ $\left.\mathcal{N}^{\perp}\right)=r(\widetilde{A} \widetilde{B})$.

$\langle 144\rangle$ Some/all of the four facts: $r[A, B]=r(A)+r(B)-r(A B), r[\widetilde{A}, B]=r(\widetilde{A})+r(B)-r(\widetilde{A} B), r[A, \widetilde{B}]=$ $r(A)+r(\widetilde{B})-r(A \widetilde{B}), r[\widetilde{A}, \widetilde{B}]=r(\widetilde{A})+r(\widetilde{B})-r(\widetilde{A} \widetilde{B})$.

$\langle 145\rangle$ Some/all of the four facts: $r[A, B]=\operatorname{Trace}(A)+\operatorname{Trace}(B)-\operatorname{Trace}(A B), r[\widetilde{A}, B]=\operatorname{Trace}(\widetilde{A})+\operatorname{Trace}(B)-$

$\operatorname{Trace}(\widetilde{A} B), r[A, \widetilde{B}]=\operatorname{Trace}(A)+\operatorname{Trace}(\widetilde{B})-\operatorname{Trace}(A \widetilde{B}), r[\widetilde{A}, \widetilde{B}]=\operatorname{Trace}(\widetilde{A})+\operatorname{Trace}(\widetilde{B})-\operatorname{Trace}(\widetilde{A} \widetilde{B})$.

$\langle 146\rangle r(A-B)=r(A)+r(B)-2 r(A B)$.

$\langle 147\rangle r(A-B)=\operatorname{Trace}(A)+\operatorname{Trace}(B)-2 \operatorname{Trace}(A B)$.

$\langle 148\rangle r\left(I_{m}-A-B\right)=m-r(A)-r(B)+2 r(A B)$.

$\langle 149\rangle r\left(I_{m}-A-B\right)=m-\operatorname{Trace}(A)-\operatorname{Trace}(B)+2 \operatorname{Trace}(A B)$. 
$\langle 150\rangle r(A-B)+r\left(I_{m}-A-B\right)=m$.

$\langle 151\rangle 2 r[A, B]=2 r(A)+2 r(B)-r(A B+B A)$.

〈152〉 Some/all of the four facts: $r\left(I_{m}-A B\right)=m-r(A B), r\left(I_{m}-\widetilde{A} B\right)=m-r(\widetilde{A} B), r\left(I_{m}-A \widetilde{B}\right)=m-r(A \widetilde{B})$, $r\left(I_{m}-\widetilde{A} \widetilde{B}\right)=m-r(\widetilde{A} \widetilde{B})$.

$\langle 153\rangle$ Some/all of the four facts: $r\left(I_{m}-A B\right)=m-\operatorname{Trace}(A B), r\left(I_{m}-\widetilde{A} B\right)=m-\operatorname{Trace}(\widetilde{A} B), r\left(I_{m}-A \widetilde{B}\right)=$ $m-\operatorname{Trace}(A \widetilde{B}), r\left(I_{m}-\widetilde{A} \widetilde{B}\right)=m-\operatorname{Trace}(\widetilde{A} \widetilde{B})$.

$\langle 154\rangle r(A-A B)=r(A)-r(A B)$ and/or $r(B-B A)=r(B)-r(B A)$.

$\langle 155\rangle r(A-A B)=\operatorname{Trace}(A)-\operatorname{Trace}(A B)$ and/or $r(B-B A)=\operatorname{Trace}(B)-\operatorname{Trace}(B A)$.

$\langle 156\rangle r(A+B-A B)=r(A)+(B)-r(A B)$.

$\langle 157\rangle r(A+B-A B)=\operatorname{Trace}(A)+\operatorname{Trace}(B)-\operatorname{Trace}(A B)$.

$\langle 158\rangle r\left(I_{m}-A B A\right)=m-r(A B A)$ and/or $r\left(I_{m}-B A B\right)=m-r(B A B)$.

$\langle 159\rangle r\left(I_{m}-A B A\right)=m-\operatorname{Trace}(A B A)$ and/or $r\left(I_{m}-B A B\right)=m-\operatorname{Trace}(B A B)$.

$\langle 160\rangle r(A-B A B)=r(A)-r(B A B)$ and/or $r(B-A B A)=r(B)-r(A B A)$.

$\langle 161\rangle r(A-B A B)=\operatorname{Trace}(A)-\operatorname{Trace}(B A B)$ and $/$ or $r(B-A B A)=\operatorname{Trace}(B)-\operatorname{Trace}(A B A)$.

$\langle 162\rangle A-B=P_{\mathcal{M} \cap \mathcal{N} \perp}-P_{\mathcal{M} \perp \cap \mathcal{N}}$.

$\langle 163\rangle \mathscr{R}(A-B)=\left(\mathcal{M} \cap \mathcal{N}^{\perp}\right) \oplus\left(\mathcal{M}^{\perp} \cap \mathcal{N}\right)$.

$\langle 164\rangle P_{\mathscr{R}(A-B)}=P_{\mathcal{M} \cap \mathcal{N} \perp}+P_{\mathcal{M} \perp \cap \mathcal{N}}$.

$\langle 165\rangle I_{m}-A-B=-P_{\mathcal{M} \cap \mathcal{N}}+P_{\mathcal{M} \perp \cap \mathcal{N} \perp}$.

$\langle 166\rangle \mathscr{R}\left(I_{m}-A-B\right)=(\mathcal{M} \cap \mathcal{N}) \oplus\left(\mathcal{M}^{\perp} \cap \mathcal{N}^{\perp}\right)$.

$\langle 167\rangle P_{\mathscr{R}\left(I_{m}-A-B\right)}=P_{\mathcal{M} \cap \mathcal{N}}+P_{\mathcal{M} \perp} \cap \mathcal{N} \perp$.

$\langle 168\rangle \mathscr{R}(A-B) \cap \mathscr{R}\left(I_{m}-A-B\right)=\{0\}$.

$\langle 169\rangle \mathbb{C}^{m}=\mathscr{R}(A-B) \oplus \mathscr{R}\left(I_{m}-A-B\right)$.

$\langle 170\rangle I_{m}=P_{\mathscr{R}(A-B)}+P_{\mathscr{R}\left(I_{m}-A-B\right)}$.

$\langle 171\rangle$ Some/all of the eight facts: $A B=P_{\mathcal{M} \cap \mathcal{N}}$ and/or $\widetilde{A} B=P_{\mathcal{M} \perp \perp \cap \mathcal{N}}, A \widetilde{B}=P_{\mathcal{M} \cap \mathcal{N} \perp}, \widetilde{A} \widetilde{B}=P_{\mathcal{M}}{ }^{\perp} \cap \mathcal{N} \perp, B A=$ $P_{\mathcal{M} \cap \mathcal{N}}, B \widetilde{A}=P_{\mathcal{M} \perp \cap \mathcal{N}}, \widetilde{B} A=P_{\mathcal{N} \cap \mathcal{N} \perp}, \widetilde{B} \widetilde{A}=P_{\mathcal{M} \perp \cap \mathcal{N} \perp}$

$\langle 172\rangle$ Some/all of the four facts: $A B+B A=2 P_{\mathcal{M} \cap \mathcal{N}}, \widetilde{A} B+B \widetilde{A}=2 P_{\mathcal{M} \perp} \cap \mathcal{N}, A \widetilde{B}+\widetilde{B} A=2 P_{\mathcal{M} \cap \mathcal{N} \perp}, \widetilde{A} \widetilde{B}+\widetilde{B} \widetilde{A}=$ $2 P_{\mathcal{M} \perp \cap \mathcal{N} \perp}$.

$\langle 173\rangle A B=P_{\mathscr{R}(A B)}$ and/or $B A=P_{\mathscr{R}(B A)}$, i.e., $A B=(A B)(A B)^{\dagger}$ and/or $B A=(B A)(B A)^{\dagger}$.

$\langle 174\rangle \widetilde{A} B=P_{\mathscr{R}(\widetilde{A} B)}$ and/or $B \widetilde{A}=P_{\mathscr{R}(B \widetilde{A})}$, i.e., $\widetilde{A} B=(\widetilde{A} B)(\widetilde{A} B)^{\dagger}$ and/or $B \widetilde{A}=(B \widetilde{A})(B \widetilde{A})^{\dagger}$.

$\langle 175\rangle A \widetilde{B}=P_{\mathscr{R}(A \widetilde{B})}$ and/or $\widetilde{B} A=P_{\mathscr{R}(\widetilde{B} A)}$, i.e., $A \widetilde{B}=(A \widetilde{B})(A \widetilde{B})^{\dagger}$ and/or $\widetilde{B} A=(\widetilde{B} A)(\widetilde{B} A)^{\dagger}$.

$\langle 176\rangle \widetilde{A} \widetilde{B}=P_{\mathscr{R}(\widetilde{A} \widetilde{B})}$ and/or $\widetilde{B} \widetilde{A}=P_{\mathscr{R}(\widetilde{B} \widetilde{A})}$, i.e., $\widetilde{A} \widetilde{B}=(\widetilde{A} \widetilde{B})(\widetilde{A} \widetilde{B})^{\dagger}$ and/or $\widetilde{B} \widetilde{A}=(\widetilde{B} \widetilde{A})(\widetilde{B} \widetilde{A})^{\dagger}$.

$\langle 177\rangle A B+B A=P_{\mathscr{R}(A B)}+P_{\mathscr{R}(B A)}$.

$\langle 178\rangle$ Some/all of the four facts: $\mathscr{R}(A B)=\mathcal{M} \cap \mathcal{N}, \mathscr{R}(\widetilde{A} B)=\mathcal{M}^{\perp} \cap \mathcal{N}, \mathscr{R}(A \widetilde{B})=\mathcal{M} \cap \mathcal{N}^{\perp}, \mathscr{R}(\widetilde{A} \widetilde{B})=\mathcal{M}^{\perp} \cap \mathcal{N}^{\perp}$.

$\langle 179\rangle$ Some/all of the four facts: $\mathscr{R}(A B+B A)=\mathcal{M} \cap \mathcal{N}, \mathscr{R}(\widetilde{A} B+B \widetilde{A})=\mathcal{M}^{\perp} \cap \mathcal{N}, \mathscr{R}(A \widetilde{B}+\widetilde{B} A)=\mathcal{M} \cap \mathcal{N} \perp$, $\mathscr{R}(\widetilde{A} \widetilde{B}+\widetilde{B} \widetilde{A})=\mathcal{M}^{\perp} \cap \mathcal{N}^{\perp}$.

$\langle 180\rangle A-A B=P_{\mathcal{\mathcal { N }} \cap \mathcal{N} \perp}$ and/or $B-B A=P_{\mathcal{M} \perp \cap \mathcal{N}}$.

$\langle 181\rangle(A-B)^{2}=P_{\mathcal{M} \cap \mathcal{N} \perp}+P_{\mathcal{M} \perp} \cap \mathcal{N}$.

$\langle 182\rangle A+B-A B=P_{\mathcal{M} \cap \mathcal{N}}+P_{\mathcal{M} \cap \mathcal{N}^{\perp}}+P_{\mathcal{M} \perp} \cap \mathcal{N}$

$\langle 183\rangle \mathscr{R}\left(I_{m}-A B\right)=\mathscr{R}\left(I_{m}-B A\right)=\left(\mathcal{M} \cap \mathcal{N}^{\perp}\right) \oplus\left(\mathcal{M}^{\perp} \cap \mathcal{N}\right) \oplus\left(\mathcal{M}^{\perp} \cap \mathcal{N}^{\perp}\right)$.

$\langle 184\rangle I_{m}-A B=P_{\mathcal{M} \cap \mathcal{N} \perp}+P_{\mathcal{M} \perp \cap \mathcal{N}}+P_{\mathcal{M} \perp \cap \mathcal{N} \perp}$.

$\langle 185\rangle \mathscr{R}(A B) \cap \mathscr{R}\left(I_{m}-A B\right)=\{0\}$ and/or $\mathscr{R}(B A) \cap \mathscr{R}\left(I_{m}-B A\right)=\{0\}$.

$\langle 186\rangle \mathscr{R}(\widetilde{A} B) \cap \mathscr{R}\left(I_{m}-\widetilde{A} B\right)=\{0\}$ and/or $\mathscr{R}(B \widetilde{A}) \cap \mathscr{R}\left(I_{m}-B \widetilde{A}\right)=\{0\}$.

$\langle 187\rangle \mathscr{R}(A \widetilde{B}) \cap \mathscr{R}\left(I_{m}-A \widetilde{B}\right)=\{0\}$ and/or $\mathscr{R}(\widetilde{B} A) \cap \mathscr{R}\left(I_{m}-\widetilde{B} A\right)=\{0\}$.

$\langle 188\rangle \mathscr{R}(\widetilde{A} \widetilde{B}) \cap \mathscr{R}\left(I_{m}-\widetilde{A} \widetilde{B}\right)=\{0\}$ and/or $\mathscr{R}(\widetilde{B} \widetilde{A}) \cap \mathscr{R}\left(I_{m}-\widetilde{B} \widetilde{A}\right)=\{0\}$.

$\langle 189\rangle \mathbb{C}^{m}=\mathscr{R}(A B) \oplus \mathscr{R}\left(I_{m}-A B\right)$ and $/$ or $\mathbb{C}^{m}=\mathscr{R}(B A) \oplus \mathscr{R}\left(I_{m}-B A\right)$.

$\langle 190\rangle \mathbb{C}^{m}=\mathscr{R}(\widetilde{A} B) \oplus \mathscr{R}\left(I_{m}-\widetilde{A} B\right)$ and $/$ or $\mathbb{C}^{m}=\mathscr{R}(B \widetilde{A}) \oplus \mathscr{R}\left(I_{m}-B \widetilde{A}\right)$.

$\langle 191\rangle \mathbb{C}^{m}=\mathscr{R}(A \widetilde{B}) \oplus \mathscr{R}\left(I_{m}-A \widetilde{B}\right)$ and $/$ or $\mathbb{C}^{m}=\mathscr{R}(\widetilde{B} A) \oplus \mathscr{R}\left(I_{m}-\widetilde{B} A\right)$.

$\langle 192\rangle \mathbb{C}^{m}=\mathscr{R}(\widetilde{A} \widetilde{B}) \oplus \mathscr{R}\left(I_{m}-\widetilde{A} \widetilde{B}\right)$ and $/$ or $\mathbb{C}^{m}=\mathscr{R}(\widetilde{B} \widetilde{A}) \oplus \mathscr{R}\left(I_{m}-\widetilde{B} \widetilde{A}\right)$.

$\langle 193\rangle I_{m}=P_{\mathscr{R}(A B)}+P_{\mathscr{R}\left(I_{m}-A B\right)}$ and/or $I_{m}=P_{\mathscr{R}(B A)}+P_{\mathscr{R}\left(I_{m}-B A\right)}$.

$\langle 194\rangle A B A=P_{\mathcal{M} \cap \mathcal{N}}$ and $/$ or $B A B=P_{\mathcal{M} \cap \mathcal{N}}$. 
$\langle 195\rangle \widetilde{A} B \widetilde{A}=P_{\mathcal{M} \perp \cap \mathcal{N}}$ and/or $B \widetilde{A} B=P_{\mathcal{M} \perp} \cap \mathcal{N}$.

$\langle 196\rangle A \widetilde{B} A=P_{\mathcal{M} \cap \mathcal{N}^{\perp}}$ and/or $\widetilde{B} A \widetilde{B}=P_{\mathcal{M} \cap \mathcal{N}^{\perp}}$.

$\langle 197\rangle \widetilde{A} \widetilde{B} \widetilde{A}=P_{\mathcal{M}^{\perp} \cap \mathcal{N}^{\perp}}$ and/or $\widetilde{B} \widetilde{A} \widetilde{B}=P_{\mathcal{M}^{\perp} \cap \mathcal{N} \perp}$.

$\langle 198\rangle A B A+B A B=2 P_{\mathcal{N} \cap \mathcal{N}}$.

$\langle 199\rangle \widetilde{A} B \widetilde{A}+B \widetilde{A} B=2 P_{\mathcal{M}^{\perp} \cap \mathcal{N}}$.

$\langle 200\rangle A \widetilde{B} A+\widetilde{B} A \widetilde{B}=2 P_{\mathcal{M} \cap \mathcal{N} \perp}$.

$\langle 201\rangle \widetilde{A} \widetilde{B} \widetilde{A}+\widetilde{B} \widetilde{A} \widetilde{B}=2 P_{\mathcal{M}^{\perp} \cap \mathcal{N}^{\perp}}$.

$\langle 202\rangle A B A+\widetilde{A} B \widetilde{A}=P_{\mathcal{M} \cap \mathcal{N}}+P_{\mathcal{M}^{\perp} \cap \mathcal{N}}$ and/or $B A B+\widetilde{B} A \widetilde{B}=P_{\mathcal{M} \cap \mathcal{N}}+P_{\mathcal{M} \cap \mathcal{N}^{\perp}}$.

$\langle 203\rangle A-B A B=P_{\mathcal{M} \cap \mathcal{N}^{\perp}}$ and/or $B-A B A=P_{\mathcal{M}^{\perp} \cap \mathcal{N}}$.

204〉 $\mathscr{R}(A-B A B)=\mathcal{M} \cap \mathcal{N}^{\perp}$ and/or $\mathscr{R}(B-A B A)=\mathcal{M}^{\perp} \cap \mathcal{N}$.

$\langle 205\rangle$ Some/all of the four facts: $\mathscr{R}(A B)=\mathscr{R}(B A), \mathscr{R}(\widetilde{A} B)=\mathscr{R}(B \widetilde{A}), \mathscr{R}(A \widetilde{B})=\mathscr{R}(\widetilde{B} A), \mathscr{R}(\widetilde{A} \widetilde{B})=\mathscr{R}(\widetilde{B} \widetilde{A})$.

〈206〉 Some/all of the four facts: $\mathscr{N}(A B)=\mathscr{N}(B A), \mathscr{N}(\widetilde{A} B)=\mathscr{N}(B \widetilde{A}), \mathscr{N}(A \widetilde{B})=\mathscr{N}(\widetilde{B} A), \mathscr{N}(\widetilde{A} \widetilde{B})=\mathscr{N}(\widetilde{B} \widetilde{A})$,

$\langle 207\rangle \mathscr{R}(A B) \oplus \mathscr{N}(A B)=\mathbb{C}^{m}$ and/or $\mathscr{R}(B A) \oplus \mathscr{N}(B A)=\mathbb{C}^{m}$.

$\langle 208\rangle \mathscr{R}(\widetilde{A} B) \oplus \mathscr{N}(\widetilde{A} B)=\mathbb{C}^{m}$ and/or $\mathscr{R}(B \widetilde{A}) \oplus \mathscr{N}(B \widetilde{A})=\mathbb{C}^{m}$.

$\langle 209\rangle \mathscr{R}(A \widetilde{B}) \oplus \mathscr{N}(A \widetilde{B})=\mathbb{C}^{m}$ and/or $\mathscr{R}(\widetilde{B} A) \oplus \mathscr{N}(\widetilde{B} A)=\mathbb{C}^{m}$.

$\langle 210\rangle \mathscr{R}(\widetilde{A} \widetilde{B}) \oplus \mathscr{N}(\widetilde{A} \widetilde{B})=\mathbb{C}^{m}$ and/or $\mathscr{R}(\widetilde{B} \widetilde{A}) \oplus \mathscr{N}(\widetilde{B} \widetilde{A})=\mathbb{C}^{m}$.

$\langle 211\rangle \mathscr{R}(A B) \subseteq \mathscr{R}(B)$ and/or $\mathscr{R}(B A) \subseteq \mathscr{R}(A)$.

$\langle 212\rangle \mathscr{R}(\widetilde{A} B) \subseteq \mathscr{R}(B)$ and/or $\mathscr{R}(B \widetilde{A}) \subseteq \mathscr{R}(A)$.

$\langle 213\rangle \mathscr{R}(A \widetilde{B}) \subseteq \mathscr{R}(\widetilde{B})$ and/or $\mathscr{R}(\widetilde{B} A) \subseteq \mathscr{R}(A)$.

$\langle 214\rangle \mathscr{R}(\widetilde{A} \widetilde{B}) \subseteq \mathscr{R}(\widetilde{B})$ and/or $\mathscr{R}(\widetilde{B} \widetilde{A}) \subseteq \mathscr{R}(\widetilde{A})$.

$\langle 215\rangle \mathscr{R}(A B)=\mathscr{R}(A) \cap \mathscr{R}(B)$ and/or $\mathscr{R}(B A)=\mathscr{R}(A) \cap \mathscr{R}(B)$.

$\langle 216\rangle \mathscr{R}(\widetilde{A} B)=\mathscr{R}(\widetilde{A}) \cap \mathscr{R}(B)$ and $/$ or $\mathscr{R}(B \widetilde{A})=\mathscr{R}(\widetilde{A}) \cap \mathscr{R}(B)$.

$\langle 217\rangle \mathscr{R}(A \widetilde{B})=\mathscr{R}(A) \cap \mathscr{R}(\widetilde{A})$ and $/$ or $\mathscr{R}(\widetilde{B} A)=\mathscr{R}(A) \cap \mathscr{R}(\widetilde{B})$.

$\langle 218\rangle \mathscr{R}(\widetilde{A} \widetilde{B})=\mathscr{R}(\widetilde{A}) \cap \mathscr{R}(\widetilde{B})$ and $/$ or $\mathscr{R}(\widetilde{B} \widetilde{A})=\mathscr{R}(\widetilde{A}) \cap \mathscr{R}(\widetilde{B})$.

$\langle 219\rangle \mathscr{R}(A B \widetilde{A})=\mathscr{R}(B A \widetilde{B})$.

$\langle 220\rangle \mathscr{N}(A B \widetilde{A})=\mathscr{N}(B A \widetilde{B})$.

Proof. The equivalences of $\langle 26\rangle-\langle 112\rangle$ and $\langle 121\rangle-\langle 220\rangle$ follow from some of (3.3), (3.4), Theorem 3.4(a), and (4.93)-(4.111). The equivalences of $\langle 29\rangle$ and $\langle 113\rangle-\langle 118\rangle$ follow from the last five equalities in Theorem $3.4(\mathrm{~d})$.

It is easy to verify that

$$
A B=B A \Leftrightarrow A \widetilde{B}=\widetilde{B} A \Leftrightarrow \widetilde{A} B=B \widetilde{A} \Leftrightarrow \widetilde{A} \widetilde{B}=\widetilde{B} \widetilde{A}
$$

for two orthogonal projectors. In consequence, replacing $A$ and $B$ with $\widetilde{A}$ and $\widetilde{B}$ in Theorem 4.1 respectively will yield other three groups of equivalent statements for the above statements to hold.

Note that

$$
A \otimes B=\left(A \otimes I_{m}\right)\left(I_{m} \otimes B\right)=\left(I_{m} \otimes B\right)\left(A \otimes I_{m}\right) .
$$

Applying Theorem 5.2 to the commutative orthogonal projectors $A \otimes I_{m}$ and $I_{m} \otimes B$ and noting that $r(A \otimes B)=$ $r(A) r(B), r\left(A \otimes I_{m}\right)=m r(A)$ and $r\left(I_{m} \otimes B\right)=m r(B)$, we obtain the following result.

Corollary 5.3. Let $\mathcal{M}$ and $\mathcal{N}$ be two linear subspaces of $\mathbb{C}^{m}$ with $A=P_{\mathcal{M}}$ and $B=P_{\mathcal{N}}$, and let

$$
\begin{aligned}
& k_{3}=r(A) r(B), \quad k_{4}=m r(A)-r(A) r(B), \quad k_{5}=m r(B)-r(A) r(B), \\
& k_{6}=m^{2}-m r(A)-m r(B)+r(A) r(B) .
\end{aligned}
$$

Then, there exists a unitary matrix $U$ such that

$$
\begin{aligned}
& A \otimes I_{m}=U \operatorname{diag}\left(I_{k_{3}+k_{4}}, 0_{k_{5}}, 0_{k_{6}}\right) U^{*}, \quad I_{m} \otimes B=U \operatorname{diag}\left(I_{k_{3}}, 0_{k_{4}}, I_{k_{5}}, 0_{k_{6}}\right) U^{*} . \\
& A \otimes I_{m}+I_{m} \otimes B=U \operatorname{diag}\left(2 I_{k_{3}}, I_{k_{4}+k_{5}}, 0_{k_{6}}\right) U^{*}, A \otimes I_{m}-I_{m} \otimes B=U \operatorname{diag}\left(0_{k_{3}}, I_{k_{4}},-I_{k_{5}}, 0_{k_{6}}\right) U^{*}, \\
& A \otimes B=U \operatorname{diag}\left(I_{k_{3}}, 0_{m^{2}-k_{3}}\right) U^{*} .
\end{aligned}
$$




\section{More on subspace inclusions and subspace equalities}

As a continuation of Corollary 2.15, we reconsider in this section subspace inequalities and subspace identities, and present many well-known and new statements for subspace inequalities and subspace identities to hold.

Theorem 6.1. Let $\mathcal{M}$ and $\mathcal{N}$ be two linear subspaces of $\mathbb{C}^{m}, A, B, \widetilde{A}$ and $\widetilde{B}$ be as given in (3.2), let $s$ be an integer, and let $k_{1}, \ldots, k_{6}$ be as given in (3.8) and (3.9). Then, the following statements are equivalent:

$\langle 1\rangle \mathcal{M} \supseteq \mathcal{N}$.

$\langle 2\rangle-\langle 37\rangle$ in Corollary 2.15.

$\langle 38\rangle \mathcal{M}=\mathscr{R}(A-B) \oplus \mathcal{N}$.

$\langle 39\rangle \mathcal{N}^{\perp}=\mathcal{M}^{\perp} \oplus \mathscr{R}(A-B)$.

$\langle 40\rangle \mathcal{M}+\mathcal{N}^{\perp}=\left(\mathcal{M}^{\perp}+\mathcal{N}\right) \oplus \mathscr{R}(A-B)$.

$\langle 41\rangle A B=B A=B$.

〈42〉 $A B+B A=2 B$.

〈43〉 $A B A=B$.

$\langle 44\rangle B A B=B$.

〈45) $A B A+B A B=2 B$.

$\langle 46\rangle(A B)^{s}=(B A)^{s}=B$ for some/all $s \geqslant 2$.

$\langle 47\rangle(A B)^{s}+(B A)^{s}=2 B$ for some/all $s \geqslant 2$.

$\langle 48\rangle(A B A)^{s}=B$ for some/all $s \geqslant 2$.

$\langle 49\rangle(B A B)^{s}=B$ for some/all $s \geqslant 2$.

$\langle 50\rangle(A B A)^{s}+(B A B)^{s}=2 B$ for some/all $s \geqslant 2$.

$\langle 51\rangle \widetilde{B} \widetilde{A}=\widetilde{B} \widetilde{A}=\widetilde{A}$.

$\langle 52\rangle \widetilde{B} \widetilde{A}+\widetilde{B} \widetilde{A}=2 \widetilde{A}$.

$\langle 53\rangle \widetilde{B} \widetilde{A} \widetilde{B}=\widetilde{A}$.

〈54〉 $\widetilde{A} \widetilde{B} \widetilde{A}=\widetilde{A}$

$\langle 55\rangle \widetilde{B} \widetilde{A} \widetilde{B}+\widetilde{A} \widetilde{B} \widetilde{A}=2 \widetilde{A}$.

$\langle 56\rangle(\widetilde{B} \widetilde{A})^{s}=(\widetilde{B} \widetilde{A})^{s}=\widetilde{A}$ for some/all $s \geqslant 2$.

$\langle 57\rangle(\widetilde{B} \widetilde{A})^{s}+(\widetilde{B} \widetilde{A})^{s}=2 \widetilde{A}$ for some/all $s \geqslant 2$.

$\langle 58\rangle(\widetilde{B} \widetilde{A} \widetilde{B})^{s}=\widetilde{A}$ for some/all $s \geqslant 2$.

$\langle 59\rangle(\widetilde{A} \widetilde{B} \widetilde{A})^{s}=\widetilde{A}$ for some/all $s \geqslant 2$.

$\langle 60\rangle(\widetilde{B} \widetilde{A} \widetilde{B})^{s}+(\widetilde{A} \widetilde{B} \widetilde{A})^{s}=2 \widetilde{A}$ for some/all $s \geqslant 2$.

$\langle 61\rangle A \succcurlyeq B$ and/or $\widetilde{B} \succcurlyeq \widetilde{A}$.

$\langle 62\rangle A-B \in \mathbb{C}_{\mathrm{OP}}^{m}$ and/or $\widetilde{B}-\widetilde{A} \in \mathbb{C}_{\mathrm{OP}}^{m}$.

$\langle 63\rangle r[A, B]=r(A)$ and $/$ or $r[\widetilde{A}, \widetilde{B}]=r(\widetilde{B})$.

664 $A=P_{\mathcal{M} \cap \mathcal{N}^{\perp}}+B$ and $/$ or $\widetilde{B}=P_{\mathcal{M} \cap \mathcal{N}^{\perp}}+\widetilde{A}$.

$\langle 65\rangle B=P_{\mathcal{M} \cap \mathcal{N}}$.

$\langle 66\rangle \widetilde{A}=P_{\mathcal{M}^{\perp} \cap \mathcal{N}^{\perp}}$.

$\langle 67\rangle$ There exists a unitary matrix $U$ such that

$$
A=U \operatorname{diag}\left(I_{k_{3}}, I_{k_{4}}, 0_{k_{6}}\right) U^{*} \text { and } B=U \operatorname{diag}\left(I_{k_{3}}, 0_{k_{4}+k_{6}}\right) U^{*} \text {. }
$$

$\langle 68\rangle$ There exists a unitary matrix $U$ such that

$$
\alpha A+\beta B=U \operatorname{diag}\left((\alpha+\beta) I_{k_{3}}, \alpha I_{k_{4}}, 0_{k_{6}}\right) U^{*}
$$

for some/all two nonzero real numbers $\alpha$ and $\beta$.

<69) $r(A B)=r(B)$ with one of the four facts: $A B=B A, A \widetilde{B}=\widetilde{B} A, \widetilde{A} B=B \widetilde{A}, \widetilde{A} \widetilde{B}=\widetilde{B} \widetilde{A}$.

$\langle 70\rangle r(A B)=r(B)$ with one of the four facts: $A B A=B A B, A \widetilde{B} A=\widetilde{B} A \widetilde{B}, \widetilde{A} B \widetilde{A}=B \widetilde{A} B, \widetilde{A} \widetilde{B} \widetilde{A}=\widetilde{B} \widetilde{A} \widetilde{B}$.

$\langle 71\rangle r(A B)=r(B)$ and $A=P_{\mathcal{M} \cap \mathcal{N}}+P_{\mathcal{M} \cap \mathcal{N} \perp}$.

$\langle 72\rangle r(A B)=r(B)$ and $A+B=2 P_{\mathcal{M} \cap \mathcal{N}}+P_{\mathcal{M} \cap \mathcal{N}^{\perp}}$. 
$\langle 73\rangle r(A B)=r(B)$ and $\widetilde{B}=P_{\mathcal{M C N \mathcal { N }}^{\perp}}+P_{\mathcal{M}^{\perp} \cap \mathcal{N}^{\perp}}$.

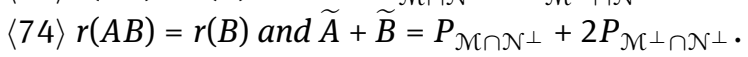

$\langle 75\rangle r(A B)=r(B)$ and $A B+B A \succcurlyeq 0$.

(76) $r(A B)=r(B)$ with one/both of the two facts: $(A-B)^{2} \succcurlyeq A-B,(B-A)^{2} \succcurlyeq B-A$.

$\langle 77\rangle r(A B)=r(B)$ and $(A-B)^{2} \in \mathbb{C}_{\mathrm{OP}}^{m}$.

$\langle 78\rangle r(A B)=r(B)$ and $(A-B)^{2^{s}+1}=A-B$ for some/all $s \geqslant 1$.

$\langle 79\rangle r(A B)=r(B)$ and $(A-B)^{\dagger}=A-B$.

<80 $r(A B)=r(B)$ with one/both of the two facts: $A(A-B)^{\dagger}=(A-B)^{\dagger} A, B(A-B)^{\dagger}=(A-B)^{\dagger} B$.

(81) $r(A B)=r(B)$ with one/both of the two facts: $A(A-B)^{\dagger}=(A-B)^{\dagger} A, B(A-B)^{\dagger}=(A-B)^{\dagger} B$.

<82 $r(A B)=r(B)$ and $(A+B)(A-B)^{\dagger}=(A-B)^{\dagger}(A+B)$.

<83 $r(A B)=r(B)$ and $A(A+B)^{\dagger} A+B(A+B)^{\dagger} B$ is an orthogonal projector.

<84 $r(A B)=r(B)$ with one/both of the two facts: $A(A+B)^{\dagger}=(A+B)^{\dagger} A, B(A+B)^{\dagger}=(A+B)^{\dagger} B$.

〈85〉 $r(A B)=r(B)$ with one/both of the two facts: $\left(I_{m}-A-B\right)^{2} \succcurlyeq I_{m}-A-B,\left(A+B-I_{m}\right)^{2} \succcurlyeq A+B-I_{m}$.

$\langle 86\rangle r(A B)=r(B)$ and $(I-A-B)^{2}$ is an orthogonal projector.

$\langle 87\rangle r(A B)=r(B)$ and $\left(I_{m}-A-B\right)^{2^{s}+1}=I_{m}-A-B$ for some/all $s \geqslant 1$.

$\langle 88\rangle r(A B)=r(B)$ and $\left(I_{m}-A-B\right)^{\dagger}=I_{m}-A-B$.

$\langle 89\rangle r(A B)=r(B)$ and $(A-B)\left(I_{m}-A-B\right)^{\dagger}=\left(I_{m}-A-B\right)^{\dagger}(A-B)$.

$\langle 90\rangle r(A B)=r(B)$ and $(A+B)(A+B)^{\dagger}=A+B-A B$.

〈91) $r(A B)=r(B)$ and $\left[(A+B)(A+B)^{\dagger}-A\right]\left[(A+B)(A+B)^{\dagger}-B\right]=0$.

$\langle 92\rangle r(A B)=r(B)$ and $(A+B)(A+B)^{\dagger}=A+\widetilde{A} B \widetilde{A}$ and $/$ or $(A+B)(A+B)^{\dagger}=B+\widetilde{B} A \widetilde{B}$.

〈93〉 $r(A B)=r(B)$ and $A+B=(A-B)(A-B)^{\dagger}+2(A B)(A B)^{\dagger}$.

〈94 $r(A B)=r(B)$ and $A \succcurlyeq(B A)(B A)^{\dagger}$ and/or $B \succcurlyeq(A B)(A B)^{\dagger}$.

$\langle 95\rangle r(A B)=r(B)$ and $A+B \succcurlyeq(A B)(A B)^{\dagger}+(B A)(B A)^{\dagger}$.

〈96) $r(A B)=r(B)$ with one/both of the two facts: $A \succcurlyeq(\widetilde{B} A)(\widetilde{B} A)^{\dagger}, B \succcurlyeq(\widetilde{A} B)(\widetilde{A} B)^{\dagger}$.

$\langle 97\rangle r(A B)=r(B)$ and $A+B \succcurlyeq(\widetilde{A} B)(\widetilde{A} B)^{\dagger}+(\widetilde{B} A)(\widetilde{B} A)^{\dagger}$.

$\langle 98\rangle r(A B)=r(B)$ and $2 A(A B+B A)^{\dagger} B=A B$ and/or $2 B(A B+B A)^{\dagger} A=B A$.

(99) $r(A B)=r(B)$ and $2 A(A B+B A)^{\dagger} B+2 B(A B+B A)^{\dagger} A=A B+B A$.

$\langle 100\rangle r(A B)=r(B)$ and $(A B)^{s} \in \mathbb{C}_{\mathrm{OP}}^{m}$ for some/all $s \geqslant 2$.

$\langle 101\rangle r(A B)=r(B)$ and $(A B)^{s}=A B$ for some/all $s \geqslant 2$.

$\langle 102\rangle r(A B)=r(B)$ and $(A B)^{s}=(B A)^{s}$ for some/all $s \geqslant 1$.

$\langle 103\rangle r(A B)=r(B)$ and $(A B)^{s}=B A$ for some/all $s \geqslant 2$.

$\langle 104\rangle r(A B)=r(B)$ and $(A B)^{s}+(B A)^{s}=A B+B A$ for some/all $s \geqslant 2$.

$\langle 105\rangle r(A B)=r(B)$ with one/both of the two facts: $(A B A)^{s} \in \mathbb{C}_{\mathrm{OP}}^{m},(B A B)^{s} \in \mathbb{C}_{\mathrm{OP}}^{m}$ for some/all $s \geqslant 2$.

$\langle 106\rangle r(A B)=r(B)$ with one/both of the two facts: $(A B A)^{s}=A B A,(B A B)^{s}=B A B$ for some/all $s \geqslant 2$.

$\langle 107\rangle r(A B)=r(B)$ with one/both of the two facts: $(A B A)^{s}=B A B,(B A B)^{s}=A B A$ for some/all $s \geqslant 2$.

$\langle 108\rangle r(A B)=r(B)$ and $(A B A)^{s}+(B A B)^{s}=A B A+B A B$ for some/all $s \geqslant 2$.

$\langle 109\rangle r(A B)=r(B)$ with one/both of the two facts: $(A B A)^{s}=A B,(B A B)^{s}=B A$ for some/all $s \geqslant 1$.

$\langle 110\rangle r(A B)=r(B)$ and $(A B A)^{s}+(B A B)^{s}=A B+B A$ for some/all $s \geqslant 1$.

$\langle 111\rangle r(A B)=r(B)$ and $\left(I_{m}-A B\right)^{s}=I_{m}-A B$ for some/all $s \geqslant 2$.

$\langle 112\rangle r(A B)=r(B)$ and $\left(I_{m}-A B\right)^{s}=I_{m}-B A$ for some/all $s \geqslant 2$.

$\langle 113\rangle r(A B)=r(B)$ and $\left(I_{m}-A B\right)^{s}=\left(I_{m}-B A\right)^{s}$ for some/all $s \geqslant 1$.

$\langle 114\rangle r(A B)=r(B)$ and $(A+B-A B)^{s}=A+B-A B$ for some/all $s \geqslant 2$.

$\langle 115\rangle r(A B)=r(B)$ and $(A+B-A B)^{\dagger}=A+B-A B$.

$\langle 116\rangle r(A B)=r(B)$ and $A+B-A B \in \mathbb{C}_{\mathrm{OP}}^{m}$.

$\langle 117\rangle r(A B)=r(B)$ and $(A B+B A)^{2 s}=2^{2 s-1}(A B+B A)$ for some $/$ all $s \geqslant 1$.

$\langle 118\rangle r(A B)=r(B)$ and $\left(I_{m}-A B-B A\right)^{2}=I_{m}$.

$\langle 119\rangle r(A B)=r(B)$ with one/both of the two facts: $I_{m}+A B \in\left\{\left(I_{m}-A B\right)^{-}\right\}, I_{m}+B A \in\left\{\left(I_{m}-B A\right)^{-}\right\}$.

$\langle 120\rangle r(A B)=r(B)$ with one/both of the two facts: $\left(I_{m}-A B A\right)^{s}=I_{m}-A B A,\left(I_{m}-B A B\right)^{s}=I_{m}-B A B$ for some/all $s \geqslant 2$.

$\langle 121\rangle r(A B)=r(B)$ and $\left(I_{m}-A B A\right)^{s}+\left(I_{m}-B A B\right)^{s}=2 I_{m}-A B A-B A B$ for some/all $s \geqslant 2$.

$\langle 122\rangle r(A B)=r(B)$ and $(A B A+B A B)^{s}=2^{s-1}(A B A+B A B)$ for some/all $s \geqslant 2$. 
$\langle 123\rangle r(A B)=r(B)$ and $\left(I_{m}-A B A-B A B\right)^{2}=I_{m}$.

$\langle 124\rangle r(A B)=r(B)$ with one/both of the two facts: $(A-B A B)^{s}=A-B A B,(B-A B A)^{S}=B-A B A$ for some/all $s \geqslant 2$.

$\langle 125\rangle r(A B)=r(B)$ and $(A-B A B)^{S}+(B-A B A)^{S}=A+B-A B A-B A B$ for some/all s $\geqslant 2$.

$\langle 126\rangle r(A B)=r(B)$ and $A-B A B \succcurlyeq 0$ and/or $B-A B A \succcurlyeq 0$.

$\langle 127\rangle r(A B)=r(B)$ and $A+B-A B A-B A B \succcurlyeq 0$.

$\langle 128\rangle r(A B)=r(B)$ with one/both of the two facts: $A-B A B \in \mathbb{C}_{\mathrm{OP}}^{m}, B-A B A \in \mathbb{C}_{\mathrm{OP}}^{m}$.

$\langle 129\rangle r(A B)=r(B)$ and $(A+B-A B A-B A B) / 2 \in \mathbb{C}_{\mathrm{OP}}^{m}$.

$\langle 130\rangle r(A B)=r(B)$ with one/both of the two facts: $A B A+\widetilde{A} B \widetilde{A} \in \mathbb{C}_{\mathrm{OP}}^{m}, B A B+\widetilde{B} A \widetilde{B} \in \mathbb{C}_{\mathrm{OP}}^{m}$.

$\langle 131\rangle r(A B)=r(B)$ with one/both of the two facts: $A B A+\widetilde{A} B \widetilde{A}=B, B A B+\widetilde{B} A \widetilde{B}=A$.

$\langle 132\rangle r(A B)=r(B)$ with one/both of the two facts: $A B+B A=2 A B A, A B+B A=2 B A B$.

$\langle 133\rangle r(A B)=r(B)$ with one/both of the two facts: $(A B)^{\dagger}=B A,(B A)^{\dagger}=A B$.

$\langle 134\rangle r(A B)=r(B)$ with one/both of the two facts: $(A B)^{\dagger}=A B,(B A)^{\dagger}=B A$.

$\langle 135\rangle r(A B)=r(B)$ and $(A B)^{\dagger}+(B A)^{\dagger}=A B+B A$.

$\langle 136\rangle r(A B)=r(B)$ and $\left[(A B)^{2}\right]^{\dagger}=\left[(A B)^{\dagger}\right]^{2}$.

$\langle 137\rangle r(A B)=r(B)$ and $\left[(A B)^{\dagger}+(B A)^{\dagger}\right] / 2 \in \mathbb{C}_{\mathrm{OP}}^{m}$.

$\langle 138\rangle r(A B)=r(B)$ with one/both of the two facts: $A B(A B)^{\dagger}=A B A, B A(B A)^{\dagger}=B A B$.

$\langle 139\rangle r(A B)=r(B)$ and $A B(A B)^{\dagger}+B A(B A)^{\dagger}=A B A+B A B$.

$\langle 140\rangle r(A B)=r(B)$ and $(A B)(A B)^{\dagger}=(B A)(B A)^{\dagger}$.

$\langle 141\rangle r(A B)=r(B)$ and $\left[(A B)(A B)^{\dagger}\right]\left[(B A)(B A)^{\dagger}\right]=\left[(B A)(B A)^{\dagger}\right]\left[(A B)(A B)^{\dagger}\right]$.

$\langle 142\rangle r(A B)=r(B)$ with one/both of the two facts: $A(\widetilde{A} \widetilde{B})^{\dagger} B=0, B(\widetilde{B} \widetilde{A})^{\dagger} A=0$.

$\langle 143\rangle r(A B)=r(B)$ and $A(\widetilde{A} \widetilde{B})^{\dagger} B+B(\widetilde{B} \widetilde{A})^{\dagger} A=0$.

$\langle 144\rangle r(A B)=r(B)$ with one/both of the two facts: $(A B)^{\#} \in \mathbb{C}_{\mathrm{OP}}^{m},(B A)^{\#} \in \mathbb{C}_{\mathrm{OP}}^{m}$.

$\langle 145\rangle r(A B)=r(B)$ and $\left[(A B)^{\#}+(B A)^{\#}\right] / 2 \in \mathbb{C}_{\mathrm{OP}}^{m}$.

$\langle 146\rangle r(A B)=r(B)$ with one/both of the two facts: $(A B)^{\#}=(A B)^{\dagger},(B A)^{\#}=(B A)^{\dagger}$.

$\langle 147\rangle r(A B)=r(B)$ and $(A B)^{\#}+(B A)^{\#}=(A B)^{\dagger}+(B A)^{\dagger}$.

$\langle 148\rangle r(A B)=r(B)$ with one/both of the two facts: $(A B)(A B)^{\#}=(A B)(A B)^{\dagger},(B A)(B A)^{\#}=(B A)(B A)^{\dagger}$.

$\langle 149\rangle r(A B)=r(B)$ and $(A B)(A B)^{\#}+(B A)(B A)^{\#}=(A B)(A B)^{\dagger}+(B A)(B A)^{\dagger}$.

$\langle 150\rangle r(A B)=r(B)$ and $(A B)^{\#}=A B$ and $/$ or $(B A)^{\#}=B A$.

$\langle 151\rangle r(A B)=r(B)$ and $(A B)^{\#}+(B A)^{\#}=A B+B A$.

$\langle 152\rangle r(A B)=r(B)$ with one/both of the two facts: $(A B A)^{\dagger}=A B A,(B A B)^{\dagger}=B A B$.

$\langle 153\rangle r(A B)=r(B)$ and $(A B A)^{\dagger}+(B A B)^{\dagger}=A B A+B A B$.

$\langle 154\rangle r(A B)=r(B)$ with one/both of the two facts: $\left(I_{m}-A B\right)^{\dagger}=I_{m}-A B,\left(I_{m}-B A\right)^{\dagger}=I_{m}-B A$.

$\langle 155\rangle r(A B)=r(B)$ with one/both of the two facts: $\left(I_{m}-A B\right)^{\dagger}=I_{m}-B A,\left(I_{m}-B A\right)^{\dagger}=I_{m}-A B$.

$\langle 156\rangle r(A B)=r(B)$ and $\left(I_{m}-A B\right)^{\dagger}+\left(I_{m}-B A\right)^{\dagger}=2 I_{m}-A B-B A$.

$\langle 157\rangle r(A B)=r(B)$ and $\left[\left(I_{m}-A B\right)^{2}\right]^{\dagger}=\left[\left(I_{m}-A B\right)^{\dagger}\right]^{2}$.

$\langle 158\rangle r(A B)=r(B)$ with one/both of the two facts: $A\left(I_{m}-B A\right)^{\dagger} B=0, B\left(I_{m}-A B\right)^{\dagger} A=0$.

$\langle 159\rangle r(A B)=r(B)$ and $A\left(I_{m}-B A\right)^{\dagger} B+B\left(I_{m}-A B\right)^{\dagger} A=0$.

$\langle 160\rangle r(A B)=r(B)$ and $\left[\left(I_{m}-A B\right)^{\dagger}+\left(I_{m}-B A\right)^{\dagger}\right] / 2 \in \mathbb{C}_{\mathrm{OP}}^{m}$.

$\langle 161\rangle r(A B)=r(B)$ with one/both of the two facts: $\left(2 I_{m}-A-B\right)\left(2 I_{m}-A-B\right)^{\dagger}=I_{m}-A B,\left(2 I_{m}-A-B\right)\left(2 I_{m}-\right.$ $A-B)^{\dagger}=I_{m}-B A$.

$\langle 162\rangle r(A B)=r(B)$ with one/both of the two facts: $\left(2 I_{m}-A B-B A\right)\left(2 I_{m}-A B-B A\right)^{\dagger}=I_{m}-A B,\left(2 I_{m}-A B-\right.$ $B A)\left(2 I_{m}-A B-B A\right)^{\dagger}=I_{m}-B A$.

$\langle 163\rangle r(A B)=r(B)$ with one/both of the two facts: $\left(I_{m}-A B A\right)^{\dagger}=I_{m}-A B A,\left(I_{m}-B A B\right)^{\dagger}=I_{m}-B A B$.

$\langle 164\rangle r(A B)=r(B)$ and $\left(I_{m}-A B A\right)^{\dagger}+\left(I_{m}-B A B\right)^{\dagger}=2 I_{m}-A B A-B A B$.

$\langle 165\rangle r(A B)=r(B)$ and $\left[(A B A)^{\dagger}+(B A B)^{\dagger}\right] / 2 \in \mathbb{C}_{\mathrm{OP}}^{m}$.

$\langle 166\rangle r(A B)=r(B)$ with one/both of the two facts: $A\left(I_{m}-A B A\right)^{\dagger} B=0, A\left(I_{m}-B A B\right)^{\dagger} B=0$.

$\langle 167\rangle r(A B)=r(B)$ and $A\left(I_{m}-A B A\right)^{\dagger} B+A\left(I_{m}-B A B\right)^{\dagger} B=0$.

$\langle 168\rangle r(A B)=r(B)$ with one/both of the two facts: $\left(2 I_{m}-A B A-B A B\right)\left(2 I_{m}-A B A-B A B\right)^{\dagger}=I_{m}-A B A$,

$\left(2 I_{m}-A B A-B A B\right)\left(2 I_{m}-A B A-B A B\right)^{\dagger}=I_{m}-B A B$.

$\langle 169\rangle r(A B)=r(B)$ and Trace $\left[(A B)^{S}\right]=\operatorname{Trace}(A B)$ for some/all $s \geqslant 2$. 
$\langle 170\rangle r(A B)=r(B)$ and $r(A B)=\operatorname{Trace}(A B)$.

$\langle 171\rangle r(A B)=r(B)$ and $\operatorname{Trace}\left[(A-B)^{2}\right]=r(A)+r(B)-2 r(A B)$.

$\langle 172\rangle r(A B)=r(B)$ and $\operatorname{Trace}\left[(A-B)^{2}\right]=\operatorname{Trace}(A)+\operatorname{Trace}(B)-2 r(A B)$.

$\langle 173\rangle r(A B)=r(B)$ and Trace $\left[(A-B)^{2 s}\right]=\operatorname{Trace}\left[(A-B)^{2}\right]$ for some/all $s \geqslant 2$.

$\langle 174\rangle r(A B)=r(B)$ and Trace $\left[\left(I_{m}-A B\right)^{s}\right]=\operatorname{Trace}\left(I_{m}-A B\right)$ for some/all $s \geqslant 2$.

$\langle 175\rangle r(A B)=r(B)$ and $\operatorname{Trace}\left[\left(I_{m}-A B A\right)^{s}\right]=\operatorname{Trace}\left(I_{m}-A B A\right)$ and $/$ or Trace $\left[\left(I_{m}-B A B\right)^{s}\right]=\operatorname{Trace}\left(I_{m}-B A B\right)$

for some/all $s \geqslant 2$.

$\langle 176\rangle r(A B)=r(B)$ and $\operatorname{dim}(\mathcal{M} \cap \mathcal{N})=r(A B)$.

$\langle 177\rangle r(A B)=r(B)$ and $r[A, B]=r(A)+r(B)-r(A B)$.

$\langle 178\rangle r(A B)=r(B)$ and $r[A, B]=\operatorname{Trace}(A)+\operatorname{Trace}(B)-\operatorname{Trace}(A B)$.

$\langle 179\rangle r(A B)=r(B)$ and $r(A-B)=r(A)+r(B)-2 r(A B)$.

$\langle 180\rangle r(A B)=r(B)$ and $r(A-B)=\operatorname{Trace}(A)+\operatorname{Trace}(B)-2 \operatorname{Trace}(A B)$.

$\langle 181\rangle r(A B)=r(B)$ and $r\left(I_{m}-A-B\right)=m-r(A)-r(B)+2 r(A B)$.

$\langle 182\rangle r(A B)=r(B)$ and $r\left(I_{m}-A-B\right)=m-\operatorname{Trace}(A)-\operatorname{Trace}(B)+2 \operatorname{Trace}(A B)$.

$\langle 183\rangle r(A B)=r(B)$ and $r(A-B)+r\left(I_{m}-A-B\right)=m$.

$\langle 184\rangle r(A B)=r(B)$ and $2 r[A, B]=2 r(A)+2 r(B)-r(A B+B A)$.

$\langle 185\rangle r(A B)=r(B)$ and $r\left(I_{m}-A B\right)=m-r(A B)$.

$\langle 186\rangle r(A B)=r(B)$ and $r\left(I_{m}-A B\right)=m-\operatorname{Trace}(A B)$.

$\langle 187\rangle r(A B)=r(B)$ and $r(A-A B)=r(A)-r(A B)$.

$\langle 188\rangle r(A B)=r(B)$ and $r(A-A B)=\operatorname{Trace}(A)-\operatorname{Trace}(A B)$.

$\langle 189\rangle r(A B)=r(B)$ and $r(A+B-A B)=r(A)+(B)-r(A B)$.

$\langle 190\rangle r(A B)=r(B)$ and $r(A+B-A B)=\operatorname{Trace}(A)+\operatorname{Trace}(B)-\operatorname{Trace}(A B)$.

$\langle 191\rangle r(A B)=r(B)$ with one/both of the two facts: $r\left(I_{m}-A B A\right)=m-r(A B A), r\left(I_{m}-B A B\right)=m-r(B A B)$.

$\langle 192\rangle r(A B)=r(B)$ with one/both of the two facts: $r\left(I_{m}-A B A\right)=m-\operatorname{Trace}(A B A), r\left(I_{m}-B A B\right)=m-$

$\operatorname{Trace}(B A B)$.

$\langle 193\rangle r(A B)=r(B)$ with one/both of the two facts: $r(A-B A B)=r(A)-r(B A B), r(B-A B A)=r(B)-r(A B A)$.

$\langle 194\rangle r(A B)=r(B)$ with one/both of the two facts: $r(A-B A B)=\operatorname{Trace}(A)-\operatorname{Trace}(B A B), r(B-A B A)=$

Trace $(B)$ - Trace $(A B A)$.

$\langle 195\rangle r(A B)=r(B)$ and $\mathscr{R}(A-B)=\mathcal{M} \cap \mathcal{N}^{\perp}$.

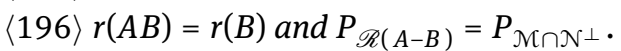

$\langle 197\rangle r(A B)=r(B)$ and $I_{m}-A-B=-P_{\mathcal{M} \cap \mathcal{N}}+P_{\mathcal{M}^{\perp} \cap \mathcal{N}^{\perp}}$.

$\langle 198\rangle r(A B)=r(B)$ and $\mathscr{R}\left(I_{m}-A-B\right)=(\mathcal{M} \cap \mathcal{N}) \oplus\left(\mathcal{M}^{\perp} \cap \mathcal{N}^{\perp}\right)$.

$\langle 199\rangle r(A B)=r(B)$ and $P_{\mathscr{R}\left(I_{m}-A-B\right)}=P_{\mathcal{M} \cap \mathcal{N}}+P_{\mathcal{M}^{\perp} \cap \mathcal{N}^{\perp}}$.

$\langle 200\rangle r(A B)=r(B)$ and $\mathscr{R}(A-B) \cap \mathscr{R}\left(I_{m}-A-B\right)=\{0\}$.

$\langle 201\rangle r(A B)=r(B)$ and $\mathbb{C}^{m}=\mathscr{R}(A-B) \oplus \mathscr{R}\left(I_{m}-A-B\right)$.

$\langle 202\rangle r(A B)=r(B)$ and $I_{m}=P_{\mathscr{R}(A-B)}+P_{\mathscr{R}\left(I_{m}-A-B\right)}$.

$\langle 203\rangle r(A B)=r(B)$ with one/both of the two facts: $A B=P_{\mathcal{M} \cap \mathcal{N}}, B A=P_{\mathcal{M N} \cap \mathcal{N}}$.

$\langle 204\rangle r(A B)=r(B)$ and $A B+B A=2 P_{\mathcal{M N} \cap \mathcal{N}}$.

$\langle 205\rangle r(A B)=r(B)$ with one/both of the two facts: $A B=(A B)(A B)^{\dagger}, B A=(B A)(B A)^{\dagger}$.

$\langle 206\rangle r(A B)=r(B)$ and $A B+B A=P_{\mathscr{R}(A B)}+P_{\mathscr{R}(B A)}$.

$\langle 207\rangle r(A B)=r(B)$ with one/both of the two facts: $\mathscr{R}(A B)=\mathcal{M} \cap \mathcal{N}, \mathscr{R}(B A)=\mathcal{M} \cap \mathcal{N}$.

$\langle 208\rangle r(A B)=r(B)$ and $\mathscr{R}(A B+B A)=\mathcal{M} \cap \mathcal{N}$.

$\langle 209\rangle r(A B)=r(B)$ and $A-A B=P_{\mathcal{M} \cap \mathcal{N}^{\perp}}$.

$\langle 210\rangle r(A B)=r(B)$ and $(A-B)^{2}=P_{\mathcal{M} \cap \mathcal{N}^{\perp}}$.

$\langle 211\rangle r(A B)=r(B)$ and $A+B-A B=P_{\mathcal{M} \cap \mathcal{N}}+P_{\mathcal{M} \cap \mathcal{N}^{\perp}}$.

212〉 $r(A B)=r(B)$ and $\mathscr{R}\left(I_{m}-A B\right)=\mathscr{R}\left(I_{m}-B A\right)=\left(\mathcal{M} \cap \mathcal{N}^{\perp}\right) \oplus\left(\mathcal{M}^{\perp} \cap \mathcal{N}^{\perp}\right)$.

$\langle 213\rangle r(A B)=r(B)$ and $I_{m}-A B=P_{\mathcal{M} \cap \mathcal{N}^{\perp}}+P_{\mathcal{M} \perp \cap \mathcal{N}^{\perp}}$.

$\langle 214\rangle r(A B)=r(B)$ with one/both of the two facts: $\mathscr{R}(A B) \cap \mathscr{R}\left(I_{m}-A B\right)=\{0\}, \mathscr{R}(B A) \cap \mathscr{R}\left(I_{m}-B A\right)=\{0\}$.

$\langle 215\rangle r(A B)=r(B)$ with one/both of the two facts: $\mathbb{C}^{m}=\mathscr{R}(A B) \oplus \mathscr{R}\left(I_{m}-A B\right), \mathbb{C}^{m}=\mathscr{R}(B A) \oplus \mathscr{R}\left(I_{m}-B A\right)$.

$\langle 216\rangle r(A B)=r(B)$ with one/both of the two facts: $I_{m}=P_{\mathscr{R}(A B)}+P_{\mathscr{R}\left(I_{m}-A B\right)}, I_{m}=P_{\mathscr{R}(B A)}+P_{\mathscr{R}\left(I_{m}-B A\right)}$.

$\langle 217\rangle r(A B)=r(B)$ with one $/$ both of the two facts: $A B A=P_{\mathcal{M} \cap \mathcal{N}}, B A B=P_{\mathcal{M} \cap \mathcal{N}}$. 
$\langle 218\rangle r(A B)=r(B)$ and $A B A+B A B=2 P_{\mathcal{M} \cap \mathcal{N}}$.

$\langle 219\rangle r(A B)=r(B)$ with one/both of the two facts: $A B A+\widetilde{A} B \widetilde{A}=P_{\mathcal{M} \cap \mathcal{N}}, B A B+\widetilde{B} A \widetilde{B}=P_{\mathcal{M} \cap \mathcal{N}}+P_{\mathcal{M} \cap \mathcal{N} \perp}$.

$\langle 220\rangle r(A B)=r(B)$ and $A-B A B=P_{\mathcal{M} \cap \mathcal{N} \perp}$.

$\langle 221\rangle \mathscr{R}(A-B A B)=\mathcal{M} \cap \mathcal{N}^{\perp}$.

$\langle 222\rangle r(A B)=r(B)$ and $\mathscr{R}(A B)=\mathscr{R}(B A)$.

$\langle 223\rangle r(A B)=r(B)$ and $\mathscr{N}(A B)=\mathscr{N}(B A)$.

$\langle 224\rangle r(A B)=r(B)$ and $\mathscr{R}(A B) \oplus \mathscr{N}(A B)=\mathbb{C}^{m}$ and/or $\mathscr{R}(B A) \oplus \mathscr{N}(B A)=\mathbb{C}^{m}$.

$\langle 225\rangle r(A B)=r(B)$ with one/both of the two facts: $\mathscr{R}(A B) \subseteq \mathscr{R}(B), \mathscr{R}(B A) \subseteq \mathscr{R}(A)$.

$\langle 226\rangle r(A B)=r(B)$ with one/both of the two facts: $\mathscr{R}(A B)=\mathscr{R}(A) \cap \mathscr{R}(B), \mathscr{R}(B A)=\mathscr{R}(A) \cap \mathscr{R}(B)$.

$\langle 227\rangle r(A B)=r(B)$ with one/both of the two facts: $\mathscr{R}(\widetilde{A} \widetilde{B})=\mathscr{N}(A) \cap \mathscr{N}(B), \mathscr{R}(\widetilde{B} \widetilde{A})=\mathscr{N}(A) \cap \mathscr{N}(B)$.

$\langle 228\rangle r(A B)=r(B)$ with one/both of the two facts: $\mathscr{R}(A B \widetilde{A})=\mathscr{R}(B A \widetilde{B}), r(A B)=r(B)$ and $\mathscr{N}(A B \widetilde{A})=\mathscr{N}(B A \widetilde{B})$.

Theorem 6.2. Let $\mathcal{M}$ and $\mathcal{N}$ be two linear subspaces of $\mathbb{C}^{m}, A, B, \widetilde{A}$ and $\widetilde{B}$ be as given in (3.2), s be an integer, and $k_{1}, \ldots, k_{6}$ be as given in (3.8) and (3.9). Then, the following statements are equivalent:

〈1) $\mathcal{M}=\mathcal{N}$.

$\langle 2\rangle-\langle 31\rangle$ in Corollary 2.16.

<32 $A=B$.

〈33 $A B=B$ and $B A=A$.

〈34〉 $A B+B A=A+B$.

$\langle 35\rangle(A B)^{S}=B$ and $(B A)^{S}=A$ for some/all $s \geqslant 2$.

$\langle 36\rangle(A B)^{S}+(B A)^{S}=A+B$ for some/all $s \geqslant 2$.

$\langle 37\rangle A B A=A$ and $B A B=B$.

$\langle 38\rangle A B A=B$ and $B A B=A$.

〈39) $A B A+B A B=A+B$.

$\langle 40\rangle(A B A)^{s}=A$ and $(B A B)^{s}=B$ for some/all $s \geqslant 2$.

$\langle 41\rangle(A B A)^{S}=B$ and $(B A B)^{S}=A$ for some/all $s \geqslant 2$.

$\langle 42\rangle(A B A)^{S}+(B A B)^{S}=A+B$ for some/all $s \geqslant 2$.

〈43〉 $\widetilde{A}=\widetilde{B}$.

〈44〉 $\widetilde{A} \widetilde{B}=\widetilde{B}$ and $\widetilde{B} \widetilde{A}=\widetilde{A}$.

〈45) $\widetilde{A} \widetilde{B}+\widetilde{B} \widetilde{A}=\widetilde{A}+\widetilde{B}$.

$\langle 46\rangle(\widetilde{A} \widetilde{B})^{s}=\widetilde{B}$ and $(\widetilde{B} \widetilde{A})^{s}=\widetilde{A}$ for some/all $s \geqslant 2$.

$\langle 47\rangle(\widetilde{A} \widetilde{B})^{s}+(\widetilde{B} \widetilde{A})^{S}=\widetilde{A}+\widetilde{B}$ for some/all $s \geqslant 2$.

〈48> $\widetilde{A} \widetilde{B} \widetilde{A}=\widetilde{B}$ and $\widetilde{B} \widetilde{A} \widetilde{B}=\widetilde{A}$.

〈49〉 $\widetilde{A} \widetilde{B} \widetilde{A}=\widetilde{A}$ and $\widetilde{B} \widetilde{A} \widetilde{B}=\widetilde{B}$.

$\langle 50\rangle \widetilde{A} \widetilde{B} \widetilde{A}+\widetilde{B} \widetilde{A} \widetilde{B}=\widetilde{A}+\widetilde{B}$.

$\langle 51\rangle(\widetilde{A} \widetilde{B} \widetilde{A})^{S}=\widetilde{B}$ and $(\widetilde{B} \widetilde{A} \widetilde{B})^{S}=\widetilde{A}$ for some/all $s \geqslant 2$.

$\langle 52\rangle(\widetilde{A} \widetilde{B} \widetilde{A})^{s}=\widetilde{A}$ and $(\widetilde{B} \widetilde{A} \widetilde{B})^{s}=\widetilde{B}$ for some/all $s \geqslant 2$.

$\langle 53\rangle(\widetilde{A} \widetilde{B} \widetilde{A})^{S}+(\widetilde{B} \widetilde{A} \widetilde{B})^{s}=\widetilde{A}+\widetilde{B}$ for some/all $s \geqslant 2$.

〈54〉 $\mathcal{M}=\mathscr{R}(A-B) \oplus \mathcal{N}$ and $\mathcal{N}=\mathscr{R}(A-B) \oplus \mathcal{M}$.

〈55〉 $\mathcal{M}^{\perp}=\mathscr{R}(A-B) \oplus \mathcal{N}^{\perp}$ and $\mathcal{N}^{\perp}=\mathcal{M}^{\perp} \oplus \mathscr{R}(A-B)$.

〈56) $2 r[A, B]=r(A)+r(B)$.

〈57) $r[A, B]=r(A)=r(B)$.

$\langle 58\rangle r[A, B]=r(A B)$.

$\langle 59\rangle 2 r[\widetilde{A}, \widetilde{B}]=r(\widetilde{A})+r(\widetilde{B})$.

$\langle 60\rangle r[\widetilde{A}, \widetilde{B}]=r(\widetilde{A})=r(\widetilde{B})$.

$\langle 61\rangle r[\widetilde{A}, \widetilde{B}]=r(\widetilde{A} \widetilde{B})$.

$\langle 62\rangle$ There exists a unitary matrix $U$ such that

$$
\alpha A+\beta B=U \operatorname{diag}\left((\alpha+\beta) I_{k_{3}}, 0_{k_{6}}\right) U^{*}
$$

for some/all two nonzero real numbers $\alpha$ and $\beta$. 
$\langle 63\rangle$ There exists a unitary matrix $U$ such that

$$
\alpha A B+\beta B A=U \operatorname{diag}\left((\alpha+\beta) I_{k_{3}}, 0_{m-k_{3}}\right) U^{*}
$$

for some/all two nonzero real numbers $\alpha$ and $\beta$.

$\langle 64\rangle$ There exists a unitary matrix $U$ such that

$$
\alpha A B A+\beta B A B=U \operatorname{diag}\left((\alpha+\beta) I_{k_{3}}, 0_{m-k_{3}}\right) U^{*}
$$

for some/all two nonzero real numbers $\alpha$ and $\beta$.

$\langle 65\rangle$ There exists a unitary matrix $U$ such that

$$
\alpha A+\beta B A B=U \operatorname{diag}\left((\alpha+\beta) I_{k_{3}}, 0_{m-k_{3}}\right) U^{*},
$$

for some/all two nonzero real numbers $\alpha$ and $\beta$, and/or there exists a unitary matrix $U$ such that

$$
\alpha B+\beta A B A=U \operatorname{diag}\left((\alpha+\beta) I_{k_{3}}, 0_{m-k_{3}}\right) U^{*}
$$

for some/all two nonzero real numbers $\alpha$ and $\beta$.

$\langle 66\rangle$ There exists a unitary matrix $U$ such that

$$
\alpha(A B)^{\dagger}+\beta(B A)^{\dagger}=U \operatorname{diag}\left((\alpha+\beta) I_{k_{3}}, 0_{m-k_{3}}\right) U^{*}
$$

for some/all two nonzero real numbers $\alpha$ and $\beta$.

$\langle 67\rangle$ There exists a unitary matrix $U$ such that

$$
\alpha(A B A)^{\dagger}+\beta(B A B)^{\dagger}=U \operatorname{diag}\left((\alpha+\beta) I_{k_{3}}, 0_{m-k_{3}}\right) U^{*}
$$

for some/all two nonzero real numbers $\alpha$ and $\beta$.

$\langle 68\rangle r(A B)=r(A)=r(B)$ with some/all of the four facts: $A B=B A, A \widetilde{B}=\widetilde{B} A, \widetilde{A} B=B \widetilde{A}, \widetilde{A} \widetilde{B}=\widetilde{B} \widetilde{A}$.

$\langle 69\rangle r(A B)=r(A)=r(B)$ with some/all of the four facts: $A B A=B A B, A \widetilde{B} A=\widetilde{B} A \widetilde{B}, \widetilde{A} B \widetilde{A}=B \widetilde{A} B, \widetilde{A} \widetilde{B} \widetilde{A}=\widetilde{B} \widetilde{A} \widetilde{B}$.

$\langle 70\rangle r(A B)=r(A)=r(B)$ with one/both of the two facts: $A B+B A \preccurlyeq A, A B+B A \preccurlyeq B$.

$\langle 71\rangle r(A B)=r(A)=r(B)$ and $A B+B A \succcurlyeq 0$.

$\langle 72\rangle r(A B)=r(A)=r(B)$ and $A(A+B)^{\dagger} A+B(A+B)^{\dagger} B \in \mathbb{C}_{\mathrm{OP}}^{m}$.

$\langle 73\rangle r(A B)=r(A)=r(B)$ with one/both of the two facts: $A(A+B)^{\dagger}=(A+B)^{\dagger} A, B(A+B)^{\dagger}=(A+B)^{\dagger} B$.

$\langle 74\rangle r(A B)=r(A)=r(B)$ with one/both of $\left(I_{m}-A-B\right)^{2} \succcurlyeq I_{m}-A-B,\left(A+B-I_{m}\right)^{2} \succcurlyeq A+B-I_{m}$.

$\langle 75\rangle r(A B)=r(A)=r(B)$ and $(I-A-B)^{2} \in \mathbb{C}_{\mathrm{OP}}^{m}$.

$\langle 76\rangle r(A B)=r(A)=r(B)$ and $\left(I_{m}-A-B\right)^{2^{s}+1}=I_{m}-A-B$ for some/all $s \geqslant 1$.

$\langle 77\rangle r(A B)=r(A)=r(B)$ and $\left(I_{m}-A-B\right)^{\dagger}=I_{m}-A-B$.

〈78 $r(A B)=r(A)=r(B)$ and $(A+B)(A+B)^{\dagger}=A+B-A B$.

$\langle 79\rangle r(A B)=r(A)=r(B)$ and $\left[(A+B)(A+B)^{\dagger}-A\right]\left[(A+B)(A+B)^{\dagger}-B\right]=0$.

$\langle 80\rangle r(A B)=r(A)=r(B)$ with one/both of the two facts: $(A+B)(A+B)^{\dagger}=A+\widetilde{A} B \widetilde{A},(A+B)(A+B)^{\dagger}=B+\widetilde{B} A \widetilde{B}$.

$\langle 81\rangle r(A B)=r(A)=r(B)$ and $(A+B)(A+B)^{\dagger}=(A B)(A B)^{\dagger}$.

(82) $r(A B)=r(A)=r(B)$ with one/both of the two facts: $A \succcurlyeq(B A)(B A)^{\dagger}, B \succcurlyeq(A B)(A B)^{\dagger}$.

$\langle 83\rangle r(A B)=r(A)=r(B)$ and $A+B \succcurlyeq(A B)(A B)^{\dagger}+(B A)(B A)^{\dagger}$.

$\langle 84\rangle r(A B)=r(A)=r(B)$ with one/both of the two facts: $A \succcurlyeq(\widetilde{B} A)(\widetilde{B} A)^{\dagger}, B \succcurlyeq(\widetilde{A} B)(\widetilde{A} B)^{\dagger}$.

$\langle 85\rangle r(A B)=r(A)=r(B)$ and $A+B \succcurlyeq(\widetilde{A} B)(\widetilde{A} B)^{\dagger}+(\widetilde{B} A)(\widetilde{B} A)^{\dagger}$.

(86) $r(A B)=r(A)=r(B)$ with one/both of the two facts: $2 A(A B+B A)^{\dagger} B=A B, 2 B(A B+B A)^{\dagger} A=B A$.

$\langle 97\rangle r(A B)=r(A)=r(B)$ and $2 A(A B+B A)^{\dagger} B+2 B(A B+B A)^{\dagger} A=A B+B A$.

$\langle 88\rangle r(A B)=r(A)=r(B)$ and $(A B)^{s} \in \mathbb{C}_{\mathrm{OP}}^{m}$ for some/all $s \geqslant 1$.

$\langle 89\rangle r(A B)=r(A)=r(B)$ and $(A B)^{s}=A B$ for some/all $s \geqslant 2$.

$\langle 90\rangle r(A B)=r(A)=r(B)$ and $(A B)^{s}=(B A)^{s}$ for some/all $s \geqslant 1$.

$\langle 91\rangle r(A B)=r(A)=r(B)$ and $(A B)^{S}=B A$ for some/all $s \geqslant 2$.

〈92) $r(A B)=r(A)=r(B)$ and $(A B)^{s}+(B A)^{s}=A B+B A$ for some/all $s \geqslant 2$.

$\langle 93\rangle r(A B)=r(A)=r(B)$ with one/both of the two facts: $(A B A)^{s} \in \mathbb{C}_{\mathrm{OP}}^{m},(B A B)^{s} \in \mathbb{C}_{\mathrm{OP}}^{m}$ for some/all $s \geqslant 1$. 
494 $r(A B)=r(A)=r(B)$ with one/both of the two facts: $(A B A)^{s}=A B A,(B A B)^{s}=B A B$ for some/all $s \geqslant 2$.

$\langle 95\rangle r(A B)=r(A)=r(B)$ with some/all of $(A B A)^{s}=B A B,(B A B)^{s}=A B A$ for some/all $s \geqslant 1$.

(96) $r(A B)=r(A)=r(B)$ and $(A B A)^{s}+(B A B)^{s}=A B A+B A B$ for some/all $s \geqslant 2$.

$\langle 97\rangle r(A B)=r(A)=r(B)$ with one/both of the two facts: $(A B A)^{s}=A B,(B A B)^{s}=B A$ for some/all $s \geqslant 1$.

(98) $r(A B)=r(A)=r(B)$ and $(A B A)^{s}+(B A B)^{s}=A B+B A$ for some/all $s \geqslant 1$.

〈99〉 $r(A B)=r(A)=r(B)$ and $\left(I_{m}-A B\right)^{s}=I_{m}-A B$ for some/all $s \geqslant 2$.

$\langle 100\rangle r(A B)=r(A)=r(B)$ and $\left(I_{m}-A B\right)^{s}=I_{m}-B A$ for some/all $s \geqslant 2$.

$\langle 101\rangle r(A B)=r(A)=r(B)$ and $\left(I_{m}-A B\right)^{s}=\left(I_{m}-B A\right)^{s}$ for some/all $s \geqslant 1$.

$\langle 102\rangle r(A B)=r(A)=r(B)$ and $(A+B-A B)^{s}=A+B-A B$ for some/all $s \geqslant 2$.

$\langle 103\rangle r(A B)=r(A)=r(B)$ and $(A+B-A B)^{\dagger}=A+B-A B$.

$\langle 104\rangle r(A B)=r(A)=r(B)$ and $A+B-A B \in \mathbb{C}_{\mathrm{OP}}^{m}$.

$\langle 105\rangle r(A B)=r(A)=r(B)$ and $(A B+B A)^{2 s}=2^{2 s-1}(A B+B A)$ for some/all $s \geqslant 1$.

$\langle 106\rangle r(A B)=r(A)=r(B)$ and $\left(I_{m}-A B-B A\right)^{2}=I_{m}$.

$\langle 107\rangle r(A B)=r(A)=r(B)$ with one/both of the two facts $I_{m}+A B \in\left\{\left(I_{m}-A B\right)^{-}\right\}, I_{m}+B A \in\left\{\left(I_{m}-B A\right)^{-}\right\}$.

$\langle 108\rangle r(A B)=r(A)=r(B)$ with one/both of the two facts: $\left(I_{m}-A B A\right)^{s}=I_{m}-A B A,\left(I_{m}-B A B\right)^{s}=I_{m}-B A B$,

for some/all $s \geqslant 2$.

$\langle 109\rangle r(A B)=r(A)=r(B)$ and $\left(I_{m}-A B A\right)^{s}+\left(I_{m}-B A B\right)^{s}=2 I_{m}-A B A-B A B$ for some/all $s \geqslant 2$.

$\langle 110\rangle r(A B)=r(A)=r(B)$ and $(A B A+B A B)^{s}=2^{s-1}(A B A+B A B)$ for some/all $s \geqslant 2$.

$\langle 111\rangle r(A B)=r(A)=r(B)$ and $\left(I_{m}-A B A-B A B\right)^{2}=I_{m}$.

112 $r(A B)=r(A)=r(B)$ with one/both of the two facts: $(A-B A B)^{S}=A-B A B,(B-A B A)^{S}=B-A B A$, for some/all $s \geqslant 2$.

$\langle 113\rangle r(A B)=r(A)=r(B)$ and $(A-B A B)^{s}+(B-A B A)^{s}=A+B-A B A-B A B$ for some/all $s \geqslant 2$.

$\langle 114\rangle r(A B)=r(A)=r(B)$ with one/both of the two facts $A-B A B \succcurlyeq 0, B-A B A \succcurlyeq 0$.

$\langle 115\rangle r(A B)=r(A)=r(B)$ and $A+B-A B A-B A B \succcurlyeq 0$.

$\langle 116\rangle r(A B)=r(A)=r(B)$ with one/both of the two facts: $A-B A B \in \mathbb{C}_{\mathrm{OP}}^{m}, B-A B A \in \mathbb{C}_{\mathrm{OP}}^{m}$.

$\langle 117\rangle r(A B)=r(A)=r(B)$ and $(A+B-A B A-B A B) / 2 \in \mathbb{C}_{\mathrm{OP}}^{m}$.

$\langle 118\rangle r(A B)=r(A)=r(B)$ and $A B A+\widetilde{A} B \widetilde{A} \in \mathbb{C}_{\mathrm{OP}}^{m}, B A B+\widetilde{B} A \widetilde{B} \in \mathbb{C}_{\mathrm{OP}}^{m}$.

$\langle 119\rangle r(A B)=r(A)=r(B)$ with one/both of the two facts: $A B A+\widetilde{A} B \widetilde{A}=B, B A B+\widetilde{B} A \widetilde{B}=A$.

$\langle 120\rangle r(A B)=r(A)=r(B)$ with one/both of the two facts: $A B \widetilde{A}+\widetilde{A} B A=0, B A \widetilde{B}+\widetilde{B} A B=0$.

$\langle 121\rangle r(A B)=r(A)=r(B)$ with one/both of the two facts: $(A B)^{\dagger}=B A,(B A)^{\dagger}=A B$.

$\langle 122\rangle r(A B)=r(A)=r(B)$ with one/both of the two facts: $(A B)^{\dagger}=A B,(B A)^{\dagger}=B A$.

$\langle 123\rangle r(A B)=r(A)=r(B)$ and $(A B)^{\dagger}+(B A)^{\dagger}=A B+B A$.

$\langle 124\rangle r(A B)=r(A)=r(B)$ and $\left[(A B)^{2}\right]^{\dagger}=\left[(A B)^{\dagger}\right]^{2}$.

$\langle 125\rangle r(A B)=r(A)=r(B)$ and $\left[(A B)^{\dagger}+(B A)^{\dagger}\right] / 2 \in \mathbb{C}_{\mathrm{OP}}^{m}$.

$\langle 126\rangle r(A B)=r(A)=r(B)$ with one/both of the two facts: $A B(A B)^{\dagger}=A B A, B A(B A)^{\dagger}=B A B$.

$\langle 127\rangle r(A B)=r(A)=r(B)$ and $A B(A B)^{\dagger}+B A(B A)^{\dagger}=A B A+B A B$.

$\langle 128\rangle r(A B)=r(A)=r(B)$ and $(A B)(B A)^{\dagger}=(B A)(A B)^{\dagger}$.

$\langle 129\rangle r(A B)=r(A)=r(B)$ and $\left[(A B)(A B)^{\dagger}\right]\left[(B A)(B A)^{\dagger}\right]=\left[(B A)(B A)^{\dagger}\right]\left[(A B)(A B)^{\dagger}\right]$.

$\langle 130\rangle r(A B)=r(A)=r(B)$ and $A(\widetilde{A} \widetilde{B})^{\dagger} B=0$, and $/$ or $r(A B)=r(A)=r(B)$ and $B(\widetilde{B} \widetilde{A})^{\dagger} A=0$.

$\langle 131\rangle r(A B)=r(A)=r(B)$ and $A(\widetilde{A} \widetilde{B})^{\dagger} B+B(\widetilde{B} \widetilde{A})^{\dagger} A=0$.

$\langle 132\rangle r(A B)=r(A)=r(B)$ with one/both of the two facts: $(A B)^{\#} \in \mathbb{C}_{\mathrm{OP}}^{m},(B A)^{\#} \in \mathbb{C}_{\mathrm{OP}}^{m}$.

$\langle 133\rangle r(A B)=r(A)=r(B)$ and $\left[(A B)^{\#}+(B A)^{\#}\right] / 2 \in \mathbb{C}_{\mathrm{OP}}^{m}$.

$\langle 134\rangle r(A B)=r(A)=r(B)$ with one/both of the two facts: $(A B)^{\#}=(A B)^{\dagger},(B A)^{\#}=(B A)^{\dagger}$.

$\langle 135\rangle r(A B)=r(A)=r(B)$ and $(A B)^{\#}+(B A)^{\#}=(A B)^{\dagger}+(B A)^{\dagger}$.

$\langle 136\rangle r(A B)=r(A)=r(B)$ with one/both of the two facts: $(A B)(A B)^{\#}=(A B)(A B)^{\dagger},(B A)(B A)^{\#}=(B A)(B A)^{\dagger}$.

$\langle 137\rangle r(A B)=r(A)=r(B)$ and $(A B)(A B)^{\#}+(B A)(B A)^{\#}=(A B)(A B)^{\dagger}+(B A)(B A)^{\dagger}$.

$\langle 138\rangle r(A B)=r(A)=r(B)$ with one/both of the two facts: $(A B)^{\#}=A B,(B A)^{\#}=B A$.

$\langle 139\rangle r(A B)=r(A)=r(B)$ and $(A B)^{\#}+(B A)^{\#}=A B+B A$.

$\langle 140\rangle r(A B)=r(A)=r(B)$ with one/both of the two facts: $(A B A)^{\dagger}=A B A,(B A B)^{\dagger}=B A B$.

$\langle 141\rangle r(A B)=r(A)=r(B)$ and $(A B A)^{\dagger}+(B A B)^{\dagger}=A B A+B A B$.

$\langle 142\rangle r(A B)=r(A)=r(B)$ with one/both of the two facts: $\left(I_{m}-A B\right)^{\dagger}=I_{m}-A B,\left(I_{m}-B A\right)^{\dagger}=I_{m}-B A$. 
$\langle 143\rangle r(A B)=r(A)=r(B)$ with one/both of the two facts: $\left(I_{m}-A B\right)^{\dagger}=I_{m}-B A,\left(I_{m}-B A\right)^{\dagger}=I_{m}-A B$.

$\langle 144\rangle r(A B)=r(A)=r(B)$ and $\left(I_{m}-A B\right)^{\dagger}+\left(I_{m}-B A\right)^{\dagger}=2 I_{m}-A B-B A$.

$\langle 145\rangle r(A B)=r(A)=r(B)$ and $\left[\left(I_{m}-A B\right)^{2}\right]^{\dagger}=\left[\left(I_{m}-A B\right)^{\dagger}\right]^{2}$.

$\langle 146\rangle r(A B)=r(A)=r(B)$ with one/both of the two facts: $A\left(I_{m}-B A\right)^{\dagger} B=0, B\left(I_{m}-A B\right)^{\dagger} A=0$.

$\langle 147\rangle r(A B)=r(A)=r(B)$ and $A\left(I_{m}-B A\right)^{\dagger} B+B\left(I_{m}-A B\right)^{\dagger} A=0$.

$\langle 148\rangle r(A B)=r(A)=r(B)$ and $\left[\left(I_{m}-A B\right)^{\dagger}+\left(I_{m}-B A\right)^{\dagger}\right] / 2 \in \mathbb{C}_{\mathrm{OP}}^{m}$.

$\langle 149\rangle r(A B)=r(A)=r(B)$ with one/both of the two facts: $\left(2 I_{m}-A-B\right)\left(2 I_{m}-A-B\right)^{\dagger}=I_{m}-A B,\left(2 I_{m}-A-\right.$

$B)\left(2 I_{m}-A-B\right)^{\dagger}=I_{m}-B A$.

$\langle 150\rangle r(A B)=r(A)=r(B)$ with one/both of the two facts: $\left(2 I_{m}-A B-B A\right)\left(2 I_{m}-A B-B A\right)^{\dagger}=I_{m}-A B$,

$\left(2 I_{m}-A B-B A\right)\left(2 I_{m}-A B-B A\right)^{\dagger}=I_{m}-B A$.

$\langle 151\rangle r(A B)=r(A)=r(B)$ with one/both of the two facts: $\left(I_{m}-A B A\right)^{\dagger}=I_{m}-A B A,\left(I_{m}-B A B\right)^{\dagger}=I_{m}-B A B$.

$\langle 152\rangle r(A B)=r(A)=r(B)$ and $\left(I_{m}-A B A\right)^{\dagger}+\left(I_{m}-B A B\right)^{\dagger}=2 I_{m}-A B A-B A B$.

$\langle 153\rangle r(A B)=r(A)=r(B)$ and $\left[(A B A)^{\dagger}+(B A B)^{\dagger}\right] / 2 \in \mathbb{C}_{\mathrm{OP}}^{m}$.

$\langle 154\rangle r(A B)=r(A)=r(B)$ with one/both of the two facts: $A\left(I_{m}-A B A\right)^{\dagger} B=0, A\left(I_{m}-B A B\right)^{\dagger} B=0$.

$\langle 155\rangle r(A B)=r(A)=r(B)$ and $A\left(I_{m}-A B A\right)^{\dagger} B+A\left(I_{m}-B A B\right)^{\dagger} B=0$.

$\langle 156\rangle r(A B)=r(A)=r(B)$ with one $/$ both $\left(2 I_{m}-A B A-B A B\right)\left(2 I_{m}-A B A-B A B\right)^{\dagger}=I_{m}-A B A,\left(2 I_{m}-A B A-\right.$

$B A B)\left(2 I_{m}-A B A-B A B\right)^{\dagger}=I_{m}-B A B$.

$\langle 157\rangle r(A B)=r(A)=r(B)$ and $\operatorname{Trace}\left[(A B)^{s}\right]=\operatorname{Trace}(A B)$ for some/all $s \geqslant 2$.

$\langle 158\rangle r(A B)=r(A)=r(B)$ and $r(A B)=\operatorname{Trace}(A B)$.

$\langle 159\rangle r(A B)=r(A)=r(B)$ and $\operatorname{Trace}\left[\left(I_{m}-A B\right)^{s}\right]=\operatorname{Trace}\left(I_{m}-A B\right)$ for some/all $s \geqslant 2$.

$\langle 160\rangle r(A B)=r(A)=r(B)$ and $\operatorname{Trace}\left[\left(I_{m}-A B A\right)^{s}\right]=\operatorname{Trace}\left(I_{m}-A B A\right)$, and/or $r(A B)=r(A)=r(B)$ and

Trace $\left[\left(I_{m}-B A B\right)^{s}\right]=\operatorname{Trace}\left(I_{m}-B A B\right)$ for some/all $s \geqslant 2$.

$\langle 161\rangle r(A B)=r(A)=r(B)$ and $r[A, B]=\operatorname{Trace}(A)+\operatorname{Trace}(B)-\operatorname{Trace}(A B)$.

$\langle 162\rangle r(A B)=r(A)=r(B)$ and $r\left(I_{m}-A-B\right)=m-r(A)-r(B)+2 r(A B)$.

$\langle 163\rangle r(A B)=r(A)=r(B)$ and $r\left(I_{m}-A-B\right)=m-\operatorname{Trace}(A)-\operatorname{Trace}(B)+2 \operatorname{Trace}(A B)$.

$\langle 164\rangle r(A B)=r(A)=r(B)$ and $r(A-B)+r\left(I_{m}-A-B\right)=m$.

$\langle 165\rangle r(A B)=r(A)=r(B)$ and $2 r[A, B]=2 r(A)+2 r(B)-r(A B+B A)$.

$\langle 166\rangle r(A B)=r(A)=r(B)$ and $r\left(I_{m}-A B\right)=m-r(A B)$.

$\langle 167\rangle r(A B)=r(A)=r(B)$ and $r\left(I_{m}-A B\right)=m-\operatorname{Trace}(A B)$.

$\langle 168\rangle r(A B)=r(A)=r(B)$ with one $/$ both $\operatorname{Trace}(A)=\operatorname{Trace}(A B)$, Trace $(B)=\operatorname{Trace}(B A)$.

$\langle 169\rangle r(A B)=r(A)=r(B)$ with one/both $r\left(I_{m}-A B A\right)=m-r(A B A), r\left(I_{m}-B A B\right)=m-r(B A B)$.

$\langle 170\rangle r(A B)=r(A)=r(B)$ with one $/$ both $r\left(I_{m}-A B A\right)=m-\operatorname{Trace}(A B A), r\left(I_{m}-B A B\right)=m-\operatorname{Trace}(B A B)$.

$\langle 171\rangle r(A B)=r(A)=r(B)$ with one $/$ both $B A B=A$, and $A B A=B$.

$\langle 172\rangle r(A B)=r(A)=r(B)$ with one $/$ both $A=P_{\mathcal{M} \cap \mathcal{N}}, B=P_{\mathcal{M} \cap \mathcal{N}}$.

$\langle 173\rangle r(A B)=r(A)=r(B)$ and $A+B=2 P_{\mathcal{M} \cap \mathcal{N}}$.

$\langle 174\rangle r(A B)=r(A)=r(B)$ and $I_{m}-A-B=-P_{\mathcal{M} \cap \mathcal{N}}+P_{\mathcal{M} \perp \cap \mathcal{N}^{\perp}}$.

$\langle 175\rangle r(A B)=r(A)=r(B)$ and $\mathscr{R}\left(I_{m}-A-B\right)=(\mathcal{M} \cap \mathcal{N}) \oplus\left(\mathcal{M}^{\perp} \cap \mathcal{N}^{\perp}\right)$.

$\langle 176\rangle r(A B)=r(A)=r(B)$ and $P_{\mathscr{R}\left(I_{m}-A-B\right)}=P_{\mathcal{M} \cap \mathcal{N}}+P_{\mathcal{M} \perp \cap \mathcal{N}^{\perp}}$.

$\langle 177\rangle r(A B)=r(A)=r(B)$ and $\mathscr{R}(A-B) \oplus \mathscr{R}\left(I_{m}-A-B\right)=\mathbb{C}^{m}$.

$\langle 178\rangle r(A B)=r(A)=r(B)$ with one $/$ both $A B=P_{\mathcal{M} \cap \mathcal{N}}, B A=P_{\mathcal{M} \cap \mathcal{N}}$.

$\langle 179\rangle r(A B)=r(A)=r(B)$ and $A B+B A=2 P_{\mathcal{M} \cap \mathcal{N}}$.

$\langle 180\rangle r(A B)=r(A)=r(B)$ with one $/$ both $\mathscr{R}(A B)=\mathcal{M} \cap \mathcal{N}, \mathscr{R}(B A)=\mathcal{M} \cap \mathcal{N}$.

$\langle 181\rangle r(A B)=r(A)=r(B)$ with one $/$ both $A B=P_{\mathscr{R}(A B)}, B A=P_{\mathscr{R}(B A)}$.

$\langle 182\rangle r(A B)=r(A)=r(B)$ and $A B+B A=P_{\mathscr{R}(A B)}+P_{\mathscr{R}(B A)}$.

$\langle 183\rangle r(A B)=r(A)=r(B)$ and $\mathscr{R}\left(I_{m}-A B\right)=\mathscr{R}\left(I_{m}-B A\right)=\mathcal{M}^{\perp} \cap \mathcal{N}^{\perp}$.

$\langle 184\rangle r(A B)=r(A)=r(B)$ and $I_{m}-A B=P_{\mathcal{M} \perp \perp \mathcal{N}^{\perp}}$.

$\langle 185\rangle r(A B)=r(A)=r(B)$ with one $/$ both $\mathscr{R}(A B) \cap \mathscr{R}\left(I_{m}-A B\right)=\{0\}, \mathscr{R}(B A) \cap \mathscr{R}\left(I_{m}-B A\right)=\{0\}$.

$\langle 186\rangle r(A B)=r(A)=r(B)$ with one/both $\mathbb{C}^{m}=\mathscr{R}(A B) \oplus \mathscr{R}\left(I_{m}-A B\right), \mathbb{C}^{m}=\mathscr{R}(B A) \oplus \mathscr{R}\left(I_{m}-B A\right)$.

$\langle 187\rangle r(A B)=r(A)=r(B)$ with one $/$ both $I_{m}=P_{\mathscr{R}(A B)}+P_{\mathscr{R}\left(I_{m}-A B\right)}, I_{m}=P_{\mathscr{R}(B A)}+P_{\mathscr{R}\left(I_{m}-B A\right)}$.

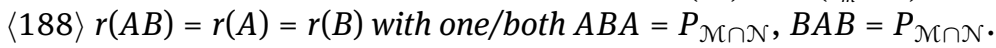

$\langle 189\rangle r(A B)=r(A)=r(B)$ and $A B A+B A B=2 P_{\mathcal{M} \cap \mathcal{N}}$. 
$\langle 190\rangle r(A B)=r(A)=r(B)$ and $\mathscr{R}(A B+B A)=\mathcal{M} \cap \mathcal{N}$.

$\langle 191\rangle r(A B)=r(A)=r(B)$ with one $/$ both $\mathscr{R}(A B)=\mathscr{R}(B A) \mathscr{N}(A B)=\mathscr{N}(B A)$.

$\langle 192\rangle r(A B)=r(A)=r(B)$ with one $/$ both $\mathscr{R}(A B) \oplus \mathscr{N}(A B)=\mathbb{C}^{m}, \mathscr{R}(B A) \oplus \mathscr{N}(B A)=\mathbb{C}^{m}$.

$\langle 193\rangle r(A B)=r(A)=r(B)$ with one/both $\mathscr{R}(A B)=\mathscr{R}(A) \cap \mathscr{R}(B), \mathscr{R}(B A)=\mathscr{R}(A) \cap \mathscr{R}(B)$.

$\langle 194\rangle r(A B)=r(A)=r(B)$ with one/both $\mathscr{R}(\widetilde{A} \widetilde{B})=\mathscr{N}(A) \cap \mathscr{N}(B), \mathscr{R}(\widetilde{B} \widetilde{A})=\mathscr{N}(A) \cap \mathscr{N}(B)$.

$\langle 195\rangle r(A B)=r(A)=r(B)$ with one $/$ both $\mathscr{R}(A B \widetilde{A})=\mathscr{R}(B A \widetilde{B}), \mathscr{N}(A B \widetilde{A})=\mathscr{N}(B A \widetilde{B})$.

\section{Equalities and inequalities for norms of orthogonal projectors}

Norms of orthogonal projectors and their operations are an important subject in both matrix theory and applications. Many equalities and inequalities for the spectrum norm and F-norms of orthogonal projectors and their operations can be found in the literature; see, e.g., $[42,71,86]$. Applying the F-norm $\|M\|_{F}^{2}=\operatorname{Trace}\left(M M^{*}\right)$ to parts of the matrix equalities in Theorem 3.4(a) and simplifying by (3.7)-(3.9), we obtain the following norm equalities and inequalities.

Theorem 7.1. Let $\mathcal{N}$ and $\mathcal{N}$ be two linear subspaces of $\mathbb{C}^{m}$, and assume that $A, B, \widetilde{A}$, and $\widetilde{B}$ in (3.2) are decomposed as (3.3)-(3.6), respectively. Then, the following norm identities hold

$$
\begin{aligned}
& \|A+B\|_{F}^{2}=2 \operatorname{Trace}\left(C^{2}\right)+2 k_{1}+4 k_{3}+k_{4}+k_{5}, \\
& \left\|A+B-I_{m}\right\|_{F}^{2}=2 \operatorname{Trace}\left(C^{2}\right)+k_{3}+k_{6} \text {, } \\
& \left\|A+B-2 P_{\mathcal{M} \cap \mathcal{N}}-P_{\mathcal{M} \cap \mathcal{N}^{\perp}}-P_{\mathcal{M}^{\perp} \cap \mathcal{N}}\right\|_{F}^{2}=2 \operatorname{Trace}\left(C^{2}\right)+2 k_{1} \text {, } \\
& \left\|P_{(\mathcal{M}+\mathcal{N})}-A-B\right\|_{F}^{2}=2 \operatorname{Trace}\left(C^{2}\right)+k_{1} \text {, } \\
& \left\|P_{(\mathcal{M}+\mathcal{N})}-A-B+A B\right\|_{F}^{2}=\operatorname{Trace}\left(C^{2}\right) \text {, } \\
& \left\|P_{(\mathcal{M}+\mathcal{N})}-A-B+P_{\mathcal{M} \cap \mathcal{N}}\right\|_{F}^{2}=2 \operatorname{Trace}\left(C^{2}\right) \text {, } \\
& \|A-B\|_{F}^{2}=2 \operatorname{Trace}\left(S^{2}\right)+k_{4}+k_{5} \text {, } \\
& \left\|A-B-P_{\mathcal{M} \cap \mathcal{N}^{\perp}}+P_{\mathcal{M}^{\perp} \cap \mathcal{N}}\right\|_{F}^{2}=2 \operatorname{Trace}\left(S^{2}\right) \text {, } \\
& \|A B\|_{F}^{2}=\|B A\|_{F}^{2}=\operatorname{Trace}\left(C^{2}\right)+k_{3} \text {, } \\
& \left\|A B-P_{\mathcal{N} \cap \mathcal{N}}\right\|_{F}^{2}=\operatorname{Trace}\left(C^{2}\right), \\
& \|A B+B A\|_{F}^{2}=2 \operatorname{Trace}\left(C^{2}+C^{4}\right)+4 k_{3}, \\
& \left\|A B+B A-2 P_{\mathcal{M} \cap \mathcal{N}}\right\|_{F}^{2}=2 \operatorname{Trace}\left(C^{2}+C^{4}\right) \text {, } \\
& \|A B-B A\|_{F}^{2}=\|A B \widetilde{A}+\widetilde{A} B A\|_{F}^{2}=\|B A \widetilde{B}+\widetilde{B} A B\|_{F}^{2}=2\|A B \widetilde{A}\|_{F}^{2}=2\|B A \widetilde{B}\|_{F}^{2}=2 \operatorname{Trace}\left(C^{2} S^{2}\right) \text {, } \\
& \left\|I_{m}-A B\right\|_{F}^{2}=\operatorname{Trace}\left(S^{2}\right)+k_{1}+k_{4}+k_{5}+k_{6} \text {, } \\
& \|A \widetilde{B}\|_{F}^{2}=\operatorname{Trace}\left(S^{2}\right)+k_{4},\|\widetilde{A} B\|_{F}^{2}=\operatorname{Trace}\left(S^{2}\right)+k_{5},\|\widetilde{A} \widetilde{B}\|_{F}^{2}=\operatorname{Trace}\left(C^{2}\right)+k_{6} \text {, } \\
& \|A B A\|_{F}^{2}=\|B A B\|_{F}^{2}=\operatorname{Trace}\left(C^{4}\right)+k_{3} \text {, } \\
& \left\|A B A-P_{\mathcal{M} \cap \mathcal{N}}\right\|_{F}^{2}=\left\|B A B-P_{\mathcal{M} \cap \mathcal{N}}\right\|_{F}^{2}=\operatorname{Trace}\left(C^{4}\right) \text {, } \\
& \|A \widetilde{B} A\|_{F}^{2}=\|\widetilde{B} A \widetilde{B}\|_{F}^{2}=\operatorname{Trace}\left(S^{4}\right)+k_{4} \text {, } \\
& \|B \widetilde{A} B\|_{F}^{2}=\|\widetilde{A} B \widetilde{A}\|_{F}^{2}=\operatorname{Trace}\left(S^{4}\right)+k_{5}, \\
& \|\widetilde{A} \widetilde{B} \widetilde{A}\|_{F}^{2}=\|\widetilde{B} \widetilde{A} \widetilde{B}\|_{F}^{2}=\operatorname{Trace}\left(C^{4}\right)+k_{6} \text {, } \\
& \|A B A+B A B\|_{F}^{2}=2 \operatorname{Trace}\left(C^{6}+C^{4}\right)+4 k_{3}, \quad\|A B A-B A B\|_{F}^{2}=2 \operatorname{Trace}\left(C^{4} S^{2}\right) \text {, } \\
& \left\|A B A+B A B-2 P_{\mathcal{M} \cap \mathcal{N}}\right\|_{F}^{2}=2 \operatorname{Trace}\left(C^{6}+C^{4}\right), \\
& \left\|I_{m}-A B A\right\|_{F}^{2}=\left\|I_{m}-B A B\right\|_{F}^{2}=\operatorname{Trace}\left(S^{4}\right)+k_{1}+k_{4}+k_{5}+k_{6} \text {, } \\
& \|A-B A B\|_{F}^{2}=-\operatorname{Trace}\left(C^{4}\right)+k_{1}+k_{4}, \quad\|B-A B A\|_{F}^{2}=-\operatorname{Trace}\left(C^{4}\right)+k_{1}+k_{5}, \\
& \|A B A+\widetilde{A} B \widetilde{A}\|_{F}^{2}=\operatorname{Trace}\left(C^{4}+S^{4}\right)+k_{3}+k_{5} \text {, }
\end{aligned}
$$


and

$$
\begin{aligned}
& \left\|(A+B)^{\dagger}\right\|_{F}^{2}=2 \operatorname{Trace}\left(2 S^{-4}+S^{-4} C^{2}\right)+4^{-1} k_{3}+k_{4}+k_{5}, \\
& \left\|\left(A+B-I_{m}\right)^{\dagger}\right\|_{F}^{2}=2 \operatorname{Trace}\left(C^{-2} S^{2}\right)+2 k_{1}+k_{3}+k_{6}, \\
& \left\|(A-B)^{\dagger}\right\|_{F}^{2}=2 \operatorname{Trace}\left(S^{-2}\right)+k_{4}+k_{5}, \\
& \left\|(A B)^{\dagger}\right\|_{F}^{2}=\left\|(B A)^{\dagger}\right\|_{F}^{2}=\operatorname{Trace}\left(C^{-2}\right)+k_{3}, \\
& \left\|(A B+B A)^{\dagger}\right\|_{F}^{2}=2 \operatorname{Trace}\left(S^{-4}+C^{-2} S^{-4}\right)+4^{-1} k_{3}, \\
& \left\|(A B-B A)^{\dagger}\right\|_{F}^{2}=\left\|(A B \widetilde{A}+\widetilde{A} B A)^{\dagger}\right\|_{F}^{2}=\left\|(B A \widetilde{B}+\widetilde{B} A B)^{\dagger}\right\|_{F}^{2}=2\left\|(A B \widetilde{A})^{\dagger}\right\|_{F}^{2} \\
& \quad=2\left\|(B A \widetilde{B})^{\dagger}\right\|_{F}^{2}=2 \operatorname{Trace}\left(C^{-2} S^{-2}\right), \\
& \left\|\left(I_{m}-A B\right)^{\dagger}\right\|_{F}^{2}=\operatorname{Trace}\left(S^{-2}+S^{-4}\right)+k_{1}+k_{4}+k_{5}+k_{6}, \\
& \left\|(A \widetilde{B})^{\dagger}\right\|_{F}^{2}=\operatorname{Trace}\left(S^{-2}\right)+k_{4},\left\|(\widetilde{A} B)^{\dagger}\right\|_{F}^{2}=\operatorname{Trace}\left(S^{-2}\right)+k_{5},\left\|(\widetilde{A} \widetilde{B})^{\dagger}\right\|_{F}^{2}=\operatorname{Trace}\left(C^{-2}\right)+k_{6}, \\
& \left\|(A B A)^{\dagger}\right\|_{F}^{2}=\left\|(B A B)^{\dagger}\right\|_{F}^{2}=\operatorname{Trace}\left(C^{-4}\right)+k_{3}, \\
& \left\|(A \widetilde{B} A)^{\dagger}\right\|_{F}^{2}=\left\|(\widetilde{B} A \widetilde{B})^{\dagger}\right\|_{F}^{2}=\operatorname{Trace}\left(S^{-4}\right)+k_{4}, \\
& \left\|(B \widetilde{A} B)^{\dagger}\right\|_{F}^{2}=\left\|(\widetilde{A} B \widetilde{A})^{\dagger}\right\|_{F}^{2}=\operatorname{Trace}\left(S^{-4}\right)+k_{5}, \\
& \left\|(\widetilde{A} \widetilde{B} \widetilde{A})^{\dagger}\right\|_{F}^{2}=\left\|(\widetilde{B} \widetilde{A} \widetilde{B})^{\dagger}\right\|_{F}^{2}=\operatorname{Trace}\left(C^{-4}\right)+k_{6}, \\
& \left\|(A B A+B A B)^{\dagger}\right\|_{F}^{2}=2 \operatorname{Trace}\left(C^{-2} S^{-4}+C^{-4} S^{-4}\right)+4^{-1} k_{3}, \\
& \left\|(A B A-B A B)^{\dagger}\right\|_{F}^{2}=2 \operatorname{Trace}\left(C^{-4} S^{-2}\right), \\
& \left\|\left(I_{m}-A B A\right)^{\dagger}\right\|_{F}^{2}=\left\|\left(I_{m}-B A B\right)^{\dagger}\right\|_{F}^{2}=\operatorname{Trace}\left(S^{-4}\right)+k_{1}+k_{4}+k_{5}+k_{6}, \\
& \left\|(A-B A B)^{\dagger}\right\|_{F}^{2}=\operatorname{Trace}\left(C^{-4} S^{-2}+C^{-2} S^{-2}\right)+k_{4}, \\
& \left\|(B-A B A)^{\dagger}\right\|_{F}^{2}=\operatorname{Trace}\left(C^{-4} S^{-2}+C^{-2} S^{-2}\right)+k_{5}, \\
& \left\|(A B A+\widetilde{A} B \widetilde{A})^{\dagger}\right\|_{F}^{2}=\operatorname{Trace}\left(C^{-4}+S^{-4}\right)+k_{3}+k_{5} .
\end{aligned}
$$

Substituting (3.8) and (3.9) into the first group of equalities in Theorem 7.1 and simplifying, we obtain the following norm inequalities.

Corollary 7.2. Let $\mathcal{M}$ and $\mathcal{N}$ be two linear subspaces of $\mathbb{C}^{m}$, and $A, B, \widetilde{A}$, and $\widetilde{B}$ be as given in (3.2). Then, the following norm inequalities hold

$$
\begin{aligned}
& 3 r(A)+3 r(B)-2 r[A, B] \leqslant\|A+B\|_{F}^{2} \leqslant r(A)+r(B)+2 r(A B), \\
& m+r(A)+r(B)-2 r[A, B] \leqslant\left\|A+B-I_{m}\right\|_{F}^{2} \leqslant m-r(A)-r(B)+2 r(A B), \\
& r(A)+r(B)-2 r(A B) \leqslant\|A-B\|_{F}^{2} \leqslant 2 r[A, B]-r(A)-r(B), \\
& r(A)+r(B)-r[A, B] \leqslant\|A B\|_{F}^{2}=\|B A\|_{F}^{2} \leqslant r(A B), \\
& 4 r(A)+4 r(B)-4 r[A, B] \leqslant\|A B+B A\|_{F}^{2} \leqslant 4 r(A B), \\
& \|A B-B A\|_{F}^{2} \leqslant \frac{1}{2} r[A, B]-\frac{1}{2} r(A)-\frac{1}{2} r(B)+\frac{1}{2} r(A B), \\
& m-r(A B) \leqslant\left\|I_{m}-A B\right\|_{F}^{2} \leqslant m-r(A)-r(B)+r[A, B], \\
& r(A)-r(A B) \leqslant\|A \widetilde{B}\|_{F}^{2} \leqslant r[A, B]-r(B), \\
& r(B)-r(A B) \leqslant\|\widetilde{A} B\|_{F}^{2} \leqslant r[A, B]-r(A), \\
& m-r[A, B] \leqslant\|\widetilde{A} \widetilde{B}\|_{F}^{2} \leqslant m-r(A)-r(B)+r(A B), \\
& r(A)+r(B)-r[A, B] \leqslant\|A B A\|_{F}^{2}=\|B A B\|_{F}^{2} \leqslant r(A B), \\
& r(A)-r(A B) \leqslant\|A \widetilde{B} A\|_{F}^{2}=\|\widetilde{B} A \widetilde{B}\|_{F}^{2} \leqslant r[A, B]-r(B), \\
& r(B)-r(A B) \leqslant\|B \widetilde{A} B\|_{F}^{2}=\|\widetilde{A} B \widetilde{A}\|_{F}^{2} \leqslant r[A, B]-r(A), \\
& m-r[A, B] \leqslant\|\widetilde{A} \widetilde{B} \widetilde{A}\|_{F}^{2}=\|\widetilde{B} \widetilde{A} \widetilde{B}\|_{F}^{2} \leqslant m-r(A)-r(B)+r(A B),
\end{aligned}
$$




$$
\begin{aligned}
& 2 r(A)+2 r(B)-2 r[A, B] \leqslant\|A B A+B A B\|_{F}^{2} \leqslant 2 r(A B), \\
& \|A B A-B A B\|_{F}^{2} \leqslant \frac{8}{27} r[A, B]-\frac{8}{27} r(A)-\frac{8}{27} r(B)+\frac{8}{27} r(A B), \\
& m-r(A B) \leqslant\left\|I_{m}-A B A\right\|_{F}^{2}=\left\|I_{m}-B A B\right\|_{F}^{2} \leqslant m-r(A)-r(B)+r[A, B], \\
& r(A)-r(A B) \leqslant\|A-B A B\|_{F}^{2} \leqslant r[A, B]-r(B), \\
& r(B)-r(A B) \leqslant\|B-A B A\|_{F}^{2} \leqslant r[A, B]-r(A), \\
& \frac{1}{2} r(A)+\frac{3}{2} r(B)-\frac{1}{2} r(A B)-\frac{1}{2} r[A, B] \leqslant\|A B A+\widetilde{A} B \widetilde{A}\|_{F}^{2} \leqslant r(B) .
\end{aligned}
$$

In particular, if $r[A, B]=r(A)+r(B)-r(A B)$, then

$$
\begin{array}{ll}
\|A+B\|_{F}^{2}=r(A)+r(B)+2 r(A B), & \left\|A+B-I_{m}\right\|_{F}^{2}=m-r(A)-r(B)+2 r(A B), \\
\|A-B\|_{F}^{2}=r(A)+r(B)-2 r(A B), & \|A B\|_{F}^{2}=\|B A\|_{F}^{2}=r(A B), \\
\|A B+B A\|_{F}^{2}=4 r(A B), & \left\|I_{m}-A B\right\|_{F}^{2}=m-r(A B), \\
\|A \widetilde{B}\|_{F}^{2}=r(A)-r(A B), & \|\widetilde{A} B\|_{F}^{2}=r(B)-r(A B), \\
\|\widetilde{A} \widetilde{B}\|_{F}^{2}=m-r(A)-r(B)+r(A B), & \|A B A\|_{F}^{2}=\|B A B\|_{F}^{2}=r(A B), \\
\|A \widetilde{B} A\|_{F}^{2}=\|\widetilde{B} A \widetilde{B}\|_{F}^{2}=r(A)-r(A B), & \|B \widetilde{A} B\|_{F}^{2}=\|\widetilde{A} B \widetilde{A}\|_{F}^{2}=r(B)-r(A B), \\
\|\widetilde{A} \widetilde{B} \widetilde{A}\|_{F}^{2}=\|\widetilde{B} \widetilde{A} \widetilde{B}\|_{F}^{2}=m-r(A)-r(B)+r(A B), & \|A B A+B A B\|_{F}^{2}=2 r(A B), \\
\left\|I_{m}-A B A\right\|_{F}^{2}=\left\|I_{m}-B A B\right\|_{F}^{2}=m-r(A B), & \|A-B A B\|_{F}^{2}=r(A)-r(A B), \\
\|B-A B A\|_{F}^{2}=r(B)-r(A B), & \|A B A+\widetilde{A} B \widetilde{A}\|_{F}^{2}=r(B) .
\end{array}
$$

\section{Characterization of an EP matrix}

The huge amount of formulas, facts, and results in the previous sections are milestones in linear algebra and matrix theory, which can serve as building materials and fundamental tools in many fields of mathematics and other disciplines. As a direct application, we consider a well-known problem of characterizing commutativity of a square matrix and its Moore-Penrose inverse.

A matrix $M \in \mathbb{C}^{m \times m}$ is said to be $\mathrm{EP}$ (or range Hermitian) if

$$
\mathscr{R}\left(M^{*}\right)=\mathscr{R}(M)
$$

holds. This concept was introduced by Schwerdtfeger in [127] and has been studied in detail by lots of authors; see e.g., [13, 23, 39, 40, 58-62, 67, 84, 85, 88, 90, 91, 104, 128, 144, 152] and references therein.

For convenience of representation, we denote by

$$
A=M M^{\dagger}, \quad B=M^{\dagger} M, \quad \widetilde{A}=I_{m}-M M^{\dagger}, \quad \widetilde{B}=I_{m}-M^{\dagger} M
$$

the four orthogonal projectors induced by $M$. Then it is obvious that

$$
r(A)=r(B)=r(M), \quad r(\widetilde{A})=r(\widetilde{B})=m-r(M), \quad r(A B)=r(B A)=r\left(M^{2}\right) .
$$

Many identifying conditions have been established for a square matrix to be EP in the literature. As a summary, we collect various known results (see [144]) on EP matrix, and present below many novel equivalent statements for a matrix to be EP without proofs.

Theorem 8.1. Let $M \in \mathbb{C}^{m \times m}$ be given, and $k_{1}, \ldots, k_{6}$ be as given in (3.15) and (3.16). Then, the following statements are equivalent:

$\langle 1\rangle M$ is $E P$ and/or $M^{*}$ is $E P$.

$\langle 2\rangle \mathscr{R}^{\perp}\left(M^{*}\right)=\mathscr{R}^{\perp}(M)$.

〈3〉 Some/all of the four facts: $\mathscr{R}(M) \subseteq \mathscr{R}\left(M^{*}\right), \mathscr{R}(M) \supseteq \mathscr{R}\left(M^{*}\right), \mathscr{R}^{\perp}(M) \subseteq \mathscr{R}^{\perp}\left(M^{*}\right), \mathscr{R}^{\perp}(M) \supseteq \mathscr{R}^{\perp}\left(M^{*}\right)$.

〈4〉 $\mathscr{R}(M)+\mathscr{R}\left(M^{*}\right)=\mathscr{R}(M)$ and/or $\mathscr{R}(M)+\mathscr{R}\left(M^{*}\right)=\mathscr{R}\left(M^{*}\right)$.

$\langle 5\rangle \mathscr{R}^{\perp}(M)+\mathscr{R}^{\perp}\left(M^{*}\right)=\mathscr{R}^{\perp}(M)$ and $/$ or $\mathscr{R}^{\perp}(M)+\mathscr{R}^{\perp}\left(M^{*}\right)=\mathscr{R}^{\perp}\left(M^{*}\right)$. 
<6> $\mathscr{R}(M)+\mathscr{R}\left(M^{*}\right)=\mathscr{R}(M) \cap \mathscr{R}\left(M^{*}\right)$ and $/$ or $\mathscr{R}^{\perp}(M)+\mathscr{R}^{\perp}\left(M^{*}\right)=\mathscr{R}^{\perp}(M) \cap \mathscr{R}^{\perp}\left(M^{*}\right)$.

〈7〉 Some/all of the four facts: $\mathscr{R}(M)=\mathscr{R}(M) \cap \mathscr{R}\left(M^{*}\right), \mathscr{R}\left(M^{*}\right)=\mathscr{R}(M) \cap \mathscr{R}\left(M^{*}\right), \mathscr{R}^{\perp}(M)=\mathscr{R}^{\perp}(M) \cap \mathscr{R}^{\perp}\left(M^{*}\right)$, $\mathscr{R}^{\perp}\left(M^{*}\right)=\mathscr{R}^{\perp}(M) \cap \mathscr{R}^{\perp}\left(M^{*}\right)$.

$\langle 8\rangle \mathscr{R}(M)=\mathscr{R}\left(M^{*}\right) \oplus\left(\mathscr{R}(M) \cap \mathscr{R}^{\perp}\left(M^{*}\right)\right)$ and $\mathscr{R}\left(M^{*}\right)=\mathscr{R}(M) \oplus\left(\mathscr{R}^{\perp}(M) \cap \mathscr{R}\left(M^{*}\right)\right)$.

$\langle 9\rangle \mathscr{R}(M)=\mathscr{R}\left(M^{*}\right) \cap\left(\mathscr{R}(M)+\mathscr{R}^{\perp}\left(M^{*}\right)\right)$ and $\mathscr{R}\left(M^{*}\right)=\mathscr{R}(M) \cap\left(\mathscr{R}^{\perp}(M)+\mathscr{R}\left(M^{*}\right)\right)$.

$\langle 10\rangle \mathscr{R}^{\perp}(M)=\mathscr{R}^{\perp}\left(M^{*}\right) \oplus\left(\mathscr{R}^{\perp}(M) \cap \mathscr{R}\left(M^{*}\right)\right)$ and $\mathscr{R}^{\perp}\left(M^{*}\right)=\mathscr{R}^{\perp}(M) \oplus\left(\mathscr{R}(M) \cap \mathscr{R}^{\perp}\left(M^{*}\right)\right)$.

$\langle 11\rangle \mathscr{R}^{\perp}(M)=\mathscr{R}^{\perp}\left(M^{*}\right) \cap\left(\mathscr{R}^{\perp}(M)+\mathscr{R}\left(M^{*}\right)\right)$ and $\mathscr{R}^{\perp}\left(M^{*}\right)=\mathscr{R}^{\perp}(M) \cap\left(\mathscr{R}(M)+\mathscr{R}^{\perp}\left(M^{*}\right)\right)$.

$\langle 12\rangle\left(\mathscr{R}(M) \cap \mathscr{R}\left(M^{*}\right)\right) \oplus\left(\mathscr{R}^{\perp}(M) \cap \mathscr{R}^{\perp}\left(M^{*}\right)\right)=\mathbb{C}^{m}$ and/or $\left(\mathscr{R}(M)+\mathscr{R}\left(M^{*}\right)\right) \cap\left(\mathscr{R}^{\perp}(M)+\mathscr{R}^{\perp}\left(M^{*}\right)\right)=\{0\}$.

$\langle 13\rangle \mathbb{C}^{m}=\mathscr{R}(M) \oplus \mathscr{R}^{\perp}\left(M^{*}\right)$ and $/$ or $\mathbb{C}^{m}=\mathscr{R}\left(M^{*}\right) \oplus \mathscr{R}^{\perp}(M)$.

$\langle 14\rangle 2 \operatorname{dim}\left(\mathscr{R}(M)+\mathscr{R}\left(M^{*}\right)\right)=\operatorname{dim}(\mathscr{R}(M))+\operatorname{dim}\left(\mathscr{R}\left(M^{*}\right)\right)$ and $/$ or $2 \operatorname{dim}\left(\mathscr{R}^{\perp}(M)+\mathscr{R}^{\perp}\left(M^{*}\right)\right)=\operatorname{dim}\left(\mathscr{R}^{\perp}(M)\right)+$ $\operatorname{dim}\left(\mathscr{R}^{\perp}\left(M^{*}\right)\right)$.

$\langle 15\rangle \operatorname{dim}\left(\mathscr{R}(M)+\mathscr{R}\left(M^{*}\right)\right)=\operatorname{dim}(\mathscr{R}(M))=\operatorname{dim}\left(\mathscr{R}\left(M^{*}\right)\right)$ and $/$ or $\operatorname{dim}\left(\mathscr{R}^{\perp}(M)+\mathscr{R}^{\perp}\left(M^{*}\right)\right)=\operatorname{dim}\left(\mathscr{R}^{\perp}(M)\right)=$ $\operatorname{dim}\left(\mathscr{R}^{\perp}\left(M^{*}\right)\right)$.

$\langle 16\rangle \operatorname{dim}\left(\mathscr{R}(M)+\mathscr{R}\left(M^{*}\right)\right)=\operatorname{dim}\left(\mathscr{R}(M) \cap \mathscr{R}\left(M^{*}\right)\right)$ and/or $\operatorname{dim}\left(\mathscr{R}^{\perp}(M)+\mathscr{R}^{\perp}\left(M^{*}\right)\right)=\operatorname{dim}\left(\mathscr{R}^{\perp}(M) \cap \mathscr{R}^{\perp}\left(M^{*}\right)\right)$.

$\langle 17\rangle \operatorname{dim}\left(\mathscr{R}(M) \cap \mathscr{R}\left(M^{*}\right)\right)=\operatorname{dim}(\mathscr{R}(M))=\operatorname{dim}\left(\mathscr{R}\left(M^{*}\right)\right)$ and $/$ or $\operatorname{dim}\left(\mathscr{R}^{\perp}(M) \cap \mathscr{R}^{\perp}\left(M^{*}\right)\right)=\operatorname{dim}\left(\mathscr{R}^{\perp}(M)\right)=$ $\operatorname{dim}\left(\mathscr{R}^{\perp}\left(M^{*}\right)\right)$.

$\langle 18\rangle \operatorname{dim}\left(\mathscr{R}(M) \cap \mathscr{R}\left(M^{*}\right)\right)+\operatorname{dim}\left(\mathscr{R}^{\perp}(M) \cap \mathscr{R}^{\perp}\left(M^{*}\right)\right)=m$.

$\langle 19\rangle \operatorname{dim}\left(\mathscr{R}(M)+\mathscr{R}\left(M^{*}\right)\right)+\operatorname{dim}\left(\mathscr{R}^{\perp}(M)+\mathscr{R}^{\perp}\left(M^{*}\right)\right)=m$.

$\langle 20\rangle \mathscr{R}(M) \cap \mathscr{R}^{\perp}\left(M^{*}\right)=\mathscr{R}^{\perp}(M) \cap \mathscr{R}\left(M^{*}\right)=\{0\}$ with some/all of the four facts: $\mathscr{R}(M)$ and $\mathscr{R}\left(M^{*}\right)$ are commutative, $\mathscr{R}(M)$ and $\mathscr{R}^{\perp}\left(M^{*}\right)$ are commutative, $\mathscr{R}^{\perp}(M)$ and $\mathscr{R}\left(M^{*}\right)$ are commutative, $\mathscr{R}^{\perp}(M)$ and $\mathscr{R}^{\perp}\left(M^{*}\right)$ are commutative.

21〉 $\mathscr{R}(M) \cap \mathscr{R}^{\perp}\left(M^{*}\right)=\mathscr{R}^{\perp}(M) \cap \mathscr{R}\left(M^{*}\right)=\{0\}$ with some/all of the four facts: $\mathscr{R}(M) \circ \mathscr{R}^{\perp}\left(M^{*}\right)=\{0\}$, $\mathscr{R}\left(M^{*}\right) \circ \mathscr{R}^{\perp}(M)=\{0\}, \mathscr{R}^{\perp}(M) \circ \mathscr{R}\left(M^{*}\right)=\{0\}, \mathscr{R}^{\perp}\left(M^{*}\right) \circ \mathscr{R}(M)=\{0\}$.

22〉 $\mathscr{R}(M) \cap \mathscr{R}^{\perp}\left(M^{*}\right)=\mathscr{R}^{\perp}(M) \cap \mathscr{R}\left(M^{*}\right)=\{0\}$ with some/all of the four facts: $\mathscr{R}(M) \oplus\left(\mathscr{R}^{\perp}(M) \cap \mathscr{R}^{\perp}\left(M^{*}\right)\right)=\mathbb{C}^{m}$, $\mathscr{R}\left(M^{*}\right) \oplus\left(\mathscr{R}^{\perp}(M) \cap \mathscr{R}^{\perp}\left(M^{*}\right)\right)=\mathbb{C}^{m}, \mathscr{R}^{\perp}(M) \oplus\left(\mathscr{R}(M) \cap \mathscr{R}\left(M^{*}\right)\right)=\mathbb{C}^{m}, \mathscr{R}^{\perp}\left(M^{*}\right) \oplus\left(\mathscr{R}(M) \cap \mathscr{R}\left(M^{*}\right)\right)=\mathbb{C}^{m}$.

〈23〉 $\mathscr{R}(M) \cap \mathscr{R}^{\perp}\left(M^{*}\right)=\mathscr{R}^{\perp}(M) \cap \mathscr{R}\left(M^{*}\right)=\{0\}$ with some/all of the four facts: $\mathscr{R}(M) \circ \mathscr{R}\left(M^{*}\right)=\mathscr{R}\left(M^{*}\right) \circ \mathscr{R}(M)$, $\mathscr{R}(M) \circ \mathscr{R}^{\perp}\left(M^{*}\right)=\mathscr{R}^{\perp}\left(M^{*}\right) \circ \mathscr{R}(M), \mathscr{R}^{\perp}(M) \circ \mathscr{R}\left(M^{*}\right)=\mathscr{R}\left(M^{*}\right) \circ \mathscr{R}^{\perp}(M), \mathscr{R}^{\perp}(M) \circ \mathscr{R}^{\perp}\left(M^{*}\right)=\mathscr{R}^{\perp}\left(M^{*}\right) \circ$ $\mathscr{R}^{\perp}(M)$

$\langle 24\rangle \mathscr{R}(M) \cap \mathscr{R}^{\perp}\left(M^{*}\right)=\mathscr{R}^{\perp}(M) \cap \mathscr{R}\left(M^{*}\right)=\{0\}$ with some/all of the four facts: $\mathscr{R}(M) \oplus\left(\mathscr{R}^{\perp}(M) \cap \mathscr{R}^{\perp}\left(M^{*}\right)\right)=$ $\mathscr{R}^{\perp}\left(M^{*}\right) \oplus\left(\mathscr{R}(M) \cap \mathscr{R}\left(M^{*}\right)\right), \mathscr{R}^{\perp}(M) \oplus\left(\mathscr{R}(M) \cap \mathscr{R}\left(M^{*}\right)\right)=\mathscr{R}\left(M^{*}\right) \oplus\left(\mathscr{R}^{\perp}(M) \cap \mathscr{R}^{\perp}\left(M^{*}\right)\right)$.

$\langle 25\rangle \mathscr{R}(M) \cap \mathscr{R}^{\perp}\left(M^{*}\right)=\mathscr{R}^{\perp}(M) \cap \mathscr{R}\left(M^{*}\right)=\{0\}$ with some/all of the four facts: $\mathscr{R}(M) \bullet \mathscr{R}\left(M^{*}\right)=\{0\}, \mathscr{R}\left(M^{*}\right) \bullet$ $\mathscr{R}(M)=\{0\}, \mathscr{R}^{\perp}(M) \bullet \mathscr{R}\left(M^{*}\right)=\{0\}, \mathscr{R}^{\perp}\left(M^{*}\right) \bullet \mathscr{R}(M)=\{0\}$.

$\langle 26\rangle \bar{M}$ is $E P$, i.e., $\mathscr{R}(\bar{M})=\mathscr{R}\left(M^{T}\right)$.

$\langle 27\rangle M^{T}$ is $E P$.

$\langle 28\rangle M M^{*} M$ is $E P$.

$\langle 29\rangle M^{\dagger}$ is $E P$.

$\langle 30\rangle r(M)=r\left(M^{2}\right)$ and $M^{\#}$ is $E P$.

$\langle 31\rangle U M U^{*}$ is $E P$ for any/some unitary matrix $U$.

$\langle 32\rangle P M P^{*}$ is EP for any/some nonsingular matrix $P$.

$\langle 33\rangle$ There exists a unitary matrix $U$ such that $U M U^{*}=\left[\begin{array}{cc}M_{1} & 0 \\ 0 & 0\end{array}\right]$, where $M_{1}$ is nonsingular.

$\langle 34\rangle$ There exists a nonsingular matrix $P$ such that $P M P^{*}=\left[\begin{array}{cc}M_{1} & 0 \\ 0 & 0\end{array}\right]$, where $M_{1}$ is nonsingular.

$\langle 35\rangle M$ can be represented as $P M P^{*}=\left[\begin{array}{cc}M_{1} & M_{1} Z^{*} \\ Z M_{1} & Z M_{1} Z^{*}\end{array}\right]$, where $P$ is a permutation matrix and $M_{1}$ is nonsingular.

〈36) There exists a matrix $V$ such that $M^{*}=M V$ and/or $M^{*}=V_{1} M, M=M^{*} V_{2}, M=V_{3} M^{*}$.

〈37〉 There exists a matrix $V$ such that $M^{\dagger}=M V$ and/or $M^{\dagger}=V M, M=M^{\dagger} V, M=V M^{\dagger}$.

$\langle 38\rangle r(M)=r\left(M^{2}\right)$ and there exists a matrix $V$ such that $M^{\#}=M^{*} V$ and/or $M^{\#}=V M^{*}$.

339 $r(M)=r\left(M^{2}\right)$ and $M^{\dagger}=M^{\#}$. 
〈40〉 $M$ commutes with $M M^{\dagger}$ and/or $M$ commutes with $M^{\dagger} M$.

〈41) $M^{\dagger}$ commutes with $M M^{\dagger}$ and/or $M^{\dagger}$ commutes with $M^{\dagger} M$.

442〉 $r(M)=r\left(M^{k}\right)$ and $M^{k}$ is EP for any/some positive number $k \geqslant 2$.

〈43〉 $A=B$ and/or $\widetilde{A}=\widetilde{B}$.

〈44〉 Some/all of the four facts: $A B=B, B A=A, \widetilde{A} \widetilde{B}=\widetilde{B}, \widetilde{B} \widetilde{A}=\widetilde{A}$.

〈45) $A B A=A$ and $B A B=B$.

〈46) $A B A=B$ and $B A B=A$.

〈47〉 $\mathscr{R}(M)=\mathscr{R}(A-B) \oplus \mathscr{R}\left(M^{*}\right)$ and $\mathscr{R}\left(M^{*}\right)=\mathscr{R}(A-B) \oplus \mathscr{R}(M)$.

$\langle 48\rangle \mathscr{R}^{\perp}(M)=\mathscr{R}(A-B) \oplus \mathscr{R}^{\perp}\left(M^{*}\right)$ and $\mathscr{R}^{\perp}\left(M^{*}\right)=\mathscr{R}^{\perp}(M) \oplus \mathscr{R}(A-B)$.

〈49) $2 r\left[M, M^{*}\right]=r(M)+r\left(M^{*}\right)$, i.e., $2 r[A, B]=r(A)+r(B)$.

$\left\langle\right.$ 50) $r\left[M, M^{*}\right]=r(M)=r\left(M^{*}\right)$, i.e., $r[A, B]=r(A)=r(B)$.

551) $r\left[M, M^{*}\right]=r\left(M^{2}\right)$, i.e., $r[A, B]=r(A B)$.

$\langle 52\rangle 2 r[\widetilde{A}, \widetilde{B}]=r(\widetilde{A})+r(\widetilde{B})$.

$\langle 53\rangle r[\widetilde{A}, \widetilde{B}]=r(\widetilde{A})=r(\widetilde{B})$.

$\langle 54\rangle r[\widetilde{A}, \widetilde{B}]=r(\widetilde{A} \widetilde{B})$.

$\langle 55\rangle$ There exists a unitary matrix $U$ such that

$$
\alpha A+\beta B=U \operatorname{diag}\left((\alpha+\beta) I_{k_{3}}, 0_{k_{6}}\right) U^{*}
$$

for some/all two nonzero real numbers $\alpha$ and $\beta$.

〈56) There exists a unitary matrix $U$ such that

$$
\alpha A B+\beta B A=U \operatorname{diag}\left((\alpha+\beta) I_{k_{3}}, 0_{m-k_{3}}\right) U^{*}
$$

for some/all two nonzero real numbers $\alpha$ and $\beta$.

$\langle 57\rangle$ There exists a unitary matrix $U$ such that

$$
\alpha A B A+\beta B A B=U \operatorname{diag}\left((\alpha+\beta) I_{k_{3}}, 0_{m-k_{3}}\right) U^{*}
$$

for some/all two nonzero real numbers $\alpha$ and $\beta$.

$\langle 58\rangle$ There exists a unitary matrix $U$ such that

$$
\alpha A+\beta B A B=U \operatorname{diag}\left((\alpha+\beta) I_{k_{3}}, 0_{m-k_{3}}\right) U^{*},
$$

for some/all two nonzero real numbers $\alpha$ and $\beta$, and/or $\mathscr{R}(M) \cap \mathscr{R}^{\perp}\left(M^{*}\right)=\mathscr{R}^{\perp}(M) \cap \mathscr{R}\left(M^{*}\right)=\{0\}$ and there exists a unitary matrix $U$ such that

$$
\alpha B+\beta A B A=U \operatorname{diag}\left((\alpha+\beta) I_{k_{3}}, 0_{m-k_{3}}\right) U^{*}
$$

for some/all two nonzero real numbers $\alpha$ and $\beta$.

〈59) There exists a unitary matrix $U$ such that

$$
\alpha(A B)^{\dagger}+\beta(B A)^{\dagger}=U \operatorname{diag}\left((\alpha+\beta) I_{k_{3}}, 0_{m-k_{3}}\right) U^{*}
$$

for some/all two nonzero real numbers $\alpha$ and $\beta$.

$\langle 60\rangle$ There exists a unitary matrix $U$ such that

$$
\alpha(A B A)^{\dagger}+\beta(B A B)^{\dagger}=U \operatorname{diag}\left((\alpha+\beta) I_{k_{3}}, 0_{m-k_{3}}\right) U^{*}
$$

for some/all two nonzero real numbers $\alpha$ and $\beta$.

〈61) $r(M)=r\left(M^{2}\right)$ and $\left(M^{2}\right)^{\dagger}=\left(M^{\dagger}\right)^{2}$.

〈62 $r(M)=r\left(M^{2}\right)$ and $M^{2}\left(M^{\dagger}\right)^{2} M^{2}=M^{2}$.

<63 $r(M)=r\left(M^{2}\right)$ and $M\left(M^{\dagger}\right)^{2} M=M M^{\#}$.

<64 There exists a polynomial $p(x)$ such that $M^{\dagger}=p(M)$ and/or there exists a polynomial $q(x)$ such that $M=$ $q\left(M^{\dagger}\right)$. 
$\langle 65\rangle\left(M M^{\dagger}\right)^{2}=M^{2}\left(M^{\dagger}\right)^{2}$ and $/$ or $\left(M^{\dagger} M\right)^{2}=\left(M^{\dagger}\right)^{2} M^{2}$.

666 $r(M)=r\left(M^{2}\right)$ and $\left(M M^{\dagger}\right)\left(M^{\dagger} M\right)=\left(M^{\dagger} M\right)\left(M M^{\dagger}\right)$.

667〉 $r(M)=r\left(M^{2}\right)$ and $\left(M M^{\dagger}\right)\left(M^{*} M\right)=\left(M^{*} M\right)\left(M M^{\dagger}\right)$ and $/$ or $\left(M^{\dagger} M\right)\left(M M^{*}\right)=\left(M M^{*}\right)\left(M^{\dagger} M\right)$.

$\langle 68\rangle r(M)=r\left(M^{2}\right)$ with one/both of the two facts: $M M^{\dagger}$ commutes with $M M^{*}+\lambda M^{*} M$ for some/all complex numbers $\lambda \neq 0, M^{\dagger} M$ commutes with $M M^{*}+\lambda M^{*} M$ for some/all complex numbers $\lambda \neq 0$.

$\langle 69\rangle M^{2} M^{\dagger}+M^{\dagger} M^{2}=2 M$.

$\langle 70\rangle M^{*} M^{\#} M+M M^{\#} M^{*}=2 M^{*}$.

$\langle 71\rangle M^{\dagger} M^{\#} M+M M^{\#} M^{\dagger}=2 M^{\dagger}$.

$\langle 72\rangle M^{2} M^{\dagger}+\left(M^{2} M^{\dagger}\right)^{*}=M+M^{*}$ and $/$ or $M^{\dagger} M^{2}+\left(M^{\dagger} M^{2}\right)^{*}=M+M^{*}$.

$\langle 73\rangle$ For any unitary matrix $U$ for which $U M U^{*}=\left[\begin{array}{cc}M_{11} & M_{12} \\ 0 & M_{22}\end{array}\right]$ with $M_{11}$ and $M_{22}$ square, $M_{11}$ and $M_{22}$ are $E P, \mathscr{R}\left(M_{12}\right) \subseteq \mathscr{R}\left(M_{11}\right)$, and $\mathscr{R}\left(M_{12}^{*}\right) \subseteq \mathscr{R}\left(M_{22}^{*}\right)$.

$\langle 74\rangle$ For any nonsingular matrix $P$ for which $P M P^{*}=\left[\begin{array}{cc}M_{11} & M_{12} \\ 0 & M_{22}\end{array}\right]$ with $M_{11}$ and $M_{22}$ square, $M_{11}$ and $M_{22}$ are $E P, \mathscr{R}\left(M_{12}\right) \subseteq \mathscr{R}\left(M_{11}\right)$, and $\mathscr{R}\left(M_{12}^{*}\right) \subseteq \mathscr{R}\left(M_{22}^{*}\right)$.

$\langle 75\rangle r(M)=r\left(M^{s+t}\right)$ and $\left(M^{s+t}\right)^{\dagger}=\left(M^{s}\right)^{\dagger}\left(M^{t}\right)^{\dagger}$ for some/all integers $s, t \geqslant 1$.

$\langle 76\rangle M^{\dagger} N=N M^{\dagger}$ whenever $M N=N M$ for certain matrix $N$.

$\langle 77\rangle M M^{\dagger}\left(M+\lambda M^{\dagger}\right)=\left(M+\lambda M^{\dagger}\right) M M^{\dagger}$ and $/$ or $M^{\dagger} M\left(M+\lambda M^{\dagger}\right)=\left(M+\lambda M^{\dagger}\right) M^{\dagger} M$ for some/all complex numbers $\lambda \neq 0$.

(78) $M M^{\dagger}\left(M+\lambda M^{*}\right)=\left(M+\lambda M^{*}\right) M M^{\dagger}$ and/or $M^{\dagger} M\left(M+\lambda M^{*}\right)=\left(M+\lambda M^{*}\right) M^{\dagger} M$ for some/all complex number $\lambda \neq 0$.

$\langle 79\rangle \mathscr{R}\left(M+\lambda M^{\dagger}\right)=\mathscr{R}\left(\lambda M+M^{3}\right)$ for some/all complex number $\lambda \neq 0$.

$\langle 80\rangle \mathscr{N}\left(M+\lambda M^{\dagger}\right)=\mathscr{N}\left(\lambda M+M^{3}\right)$ for some/all complex number $\lambda \neq 0$.

$\langle 81\rangle r(M)=r\left(M^{2}\right)$ and $M M^{\#}$ is Hermitian.

<82 $r(M)=r\left(M^{2}\right)$ with one/both of the two facts: $M M^{\dagger}=M M^{\#}, M^{\dagger} M=M^{\#} M$.

<83〉 $r(M)=r\left(M^{2}\right)$ and $M M^{\#} M^{*}=M^{*} M^{\#} M$.

(84) $r(M)=r\left(M^{2}\right)$ and $M M^{\#} M^{\dagger}=M^{\dagger} M^{\#} M$.

$\langle 85\rangle r(M)=r\left(M^{2}\right)$ with one/both of the two facts: $\left(M M^{*}\right)\left(M M^{\#}\right)=\left(M M^{\#}\right)\left(M M^{*}\right),\left(M^{*} M\right)\left(M^{\#} M\right)=$ $\left(M^{\#} M\right)\left(M^{*} M\right)$.

$\langle 86\rangle r(M)=r\left(M^{2}\right)$ and $\left(M M^{\#}\right)\left(M M^{*}+\lambda M^{*} M\right)=\left(M M^{*}+\lambda M^{*} M\right)\left(M M^{\#}\right)$ for some/all complex numbers $\lambda \neq 0$.

$\langle 87\rangle\left\{r(M)=r\left(M^{2}\right)\right.$ with one/both of the two facts: $\left(M M^{\dagger}\right)\left(M^{*} M\right)^{\dagger}=\left(M^{*} M\right)^{\dagger}\left(M M^{\dagger}\right),\left(M^{\dagger} M\right)\left(M M^{*}\right)^{\dagger}=$ $\left(M M^{*}\right)^{\dagger}\left(M^{\dagger} M\right)$.

$\langle 88\rangle r(M)=r\left(M^{k}\right)$ and $M^{k} M^{\dagger}=M^{\dagger} M^{k}$ for some/all integers $k \geqslant 2$.

<89 $r(M)=r\left(M^{k}\right)$ and $M^{k+1} M^{\dagger}+M^{\dagger} M^{k+1}=2 M^{k}$ for some/all integers $k \geqslant 1$.

〈90) $r(M)=r\left(M^{k}\right)$ and $M^{k+1} M^{\dagger}+\left(M^{\dagger} M^{k+1}\right)^{*}=M^{k}+\left(M^{k}\right)^{*}$ for some/all integers $k \geqslant 1$.

〈91) $r\left(M^{2}\right)=r(M)$ with some/all of the four facts: $A B=B A, A \widetilde{B}=\widetilde{B} A, \widetilde{A} B=B \widetilde{A}, \widetilde{A} \widetilde{B}=\widetilde{B} \widetilde{A}$.

〈92〉 $r\left(M^{2}\right)=r(M)$ with some/all of the four facts: $A B A=B A B, A \widetilde{B} A=\widetilde{B} A \widetilde{B}, \widetilde{A} B \widetilde{A}=B \widetilde{A} B, \widetilde{A} \widetilde{B} \widetilde{A}=\widetilde{B} \widetilde{A} \widetilde{B}$.

〈93> $r\left(M^{2}\right)=r(M)$ with one/both of the two facts: $A B+B A \preccurlyeq A, A B+B A \preccurlyeq B$.

〈94 $r\left(M^{2}\right)=r(M)$ and $A B+B A \succcurlyeq 0$.

〈95> $r\left(M^{2}\right)=r(M)$ and $A(A+B)^{\dagger} A+B(A+B)^{\dagger} B \in \mathbb{C}_{\mathrm{OP}}^{m}$.

〈96) $r\left(M^{2}\right)=r(M)$ with one/both of the two facts: $A(A+B)^{\dagger}=(A+B)^{\dagger} A, B(A+B)^{\dagger}=(A+B)^{\dagger} B$.

〈97〉 $r\left(M^{2}\right)=r(M)$ with one/both of the two facts: $\left(I_{m}-A-B\right)^{2} \succcurlyeq I_{m}-A-B,\left(A+B-I_{m}\right)^{2} \succcurlyeq A+B-I_{m}$.

498> $r\left(M^{2}\right)=r(M)$ and $(I-A-B)^{2} \in \mathbb{C}_{\mathrm{OP}}^{m}$.

$\langle 99\rangle r\left(M^{2}\right)=r(M)$ and $\left(I_{m}-A-B\right)^{2^{s}+1}=I_{m}-A-B$ for one/both of $s \geqslant 1$.

$\langle 100\rangle r\left(M^{2}\right)=r(M)$ and $\left(I_{m}-A-B\right)^{\dagger}=I_{m}-A-B$.

$\langle 101\rangle r\left(M^{2}\right)=r(M)$ with one/both of the two facts: $(A+B)(A+B)^{\dagger}=A+B-A B,(A+B)(A+B)^{\dagger}=A+B-B A$.

$\langle 102\rangle r\left(M^{2}\right)=r(M)$ and $\left[(A+B)(A+B)^{\dagger}-A\right]\left[(A+B)(A+B)^{\dagger}-B\right]=0$.

$\langle 103\rangle r\left(M^{2}\right)=r(M)$ with one/both of the two facts: $(A+B)(A+B)^{\dagger}=A+\widetilde{A} B \widetilde{A},(A+B)(A+B)^{\dagger}=B+\widetilde{B} A \widetilde{B}$.

$\langle 104\rangle r\left(M^{2}\right)=r(M)$ with some/all of the four facts: $(A+B)(A+B)^{\dagger}=(A B)(A B)^{\dagger},(A+B)(A+B)^{\dagger}=(A B)^{\dagger}(A B)$,

$(A+B)(A+B)^{\dagger}=(B A)(B A)^{\dagger},(A+B)(A+B)^{\dagger}=(B A)^{\dagger}(B A)$. 
$\langle 105\rangle r\left(M^{2}\right)=r(M)$ with some/all of the four facts: $A \succcurlyeq(B A)(B A)^{\dagger}, A \succcurlyeq(A B)^{\dagger}(A B), B \succcurlyeq(A B)(A B)^{\dagger}, B \succcurlyeq$ $(B A)^{\dagger}(B A)$.

$\langle 106\rangle r\left(M^{2}\right)=r(M)$ with one/both of the two facts: $A+B \succcurlyeq(A B)(A B)^{\dagger}+(B A)(B A)^{\dagger}, A+B \succcurlyeq(A B)^{\dagger}(A B)+$ $(B A)^{\dagger}(B A)$.

$\langle 107\rangle r\left(M^{2}\right)=r(M)$ with some/all of the four facts: $A \succcurlyeq(\widetilde{B} A)(\widetilde{B} A)^{\dagger}, A \succcurlyeq(A \widetilde{B})^{\dagger}(A \widetilde{B}), B \succcurlyeq(\widetilde{A} B)(\widetilde{A} B)^{\dagger}, B \succcurlyeq$ $(B \widetilde{A})^{\dagger}(B \widetilde{A})$.

$\langle 108\rangle r\left(M^{2}\right)=r(M)$ with one/both of the two facts: $A+B \succcurlyeq(\widetilde{A} B)(\widetilde{A} B)^{\dagger}+(\widetilde{B} A)(\widetilde{B} A)^{\dagger}, A+B \succcurlyeq(B \widetilde{A})^{\dagger}(B \widetilde{A})+$ $(A \widetilde{B})^{\dagger}(A \widetilde{B})$.

$\langle 109\rangle r\left(M^{2}\right)=r(M)$ with one/both of the two facts: $2 A(A B+B A)^{\dagger} B=A B, 2 B(A B+B A)^{\dagger} A=B A$.

$\langle 110\rangle r\left(M^{2}\right)=r(M)$ and $2 A(A B+B A)^{\dagger} B+2 B(A B+B A)^{\dagger} A=A B+B A$.

$\langle 111\rangle r\left(M^{2}\right)=r(M)$ and $(A B)^{s} \in \mathbb{C}_{\mathrm{OP}}^{m}$ for one/both of $s \geqslant 1$.

$\langle 112\rangle r\left(M^{2}\right)=r(M)$ and $(A B)^{S}=A B$ for one/both of $s \geqslant 2$.

$\langle 113\rangle r\left(M^{2}\right)=r(M)$ and $(A B)^{s}=(B A)^{s}$ for some/all of $s \geqslant 1$.

$\langle 114\rangle r\left(M^{2}\right)=r(M)$ and $(A B)^{s}=B A$ for some/all of $s \geqslant 2$.

$\langle 115\rangle r\left(M^{2}\right)=r(M)$ and $(A B)^{s}+(B A)^{s}=A B+B A$ for some/all of $s \geqslant 2$.

$\langle 116\rangle r\left(M^{2}\right)=r(M)$ with one/both of the two facts: $(A B A)^{s} \in \mathbb{C}_{\mathrm{OP}}^{m},(B A B)^{s} \in \mathbb{C}_{\mathrm{OP}}^{m}$ for some/all of $s \geqslant 1$.

$\langle 117\rangle r\left(M^{2}\right)=r(M)$ with one/both of the two facts: $(A B A)^{s}=A B A,(B A B)^{s}=B A B$ for some/all $s \geqslant 2$.

$\langle 118\rangle r\left(M^{2}\right)=r(M)$ with one/both of the two facts: $(A B A)^{s}=B A B,(B A B)^{s}=A B A$ for some/all $s \geqslant 1$.

$\langle 119\rangle r\left(M^{2}\right)=r(M)$ and $(A B A)^{s}+(B A B)^{s}=A B A+B A B$ for some/all of $s \geqslant 2$.

$\langle 120\rangle r\left(M^{2}\right)=r(M)$ with one/both of the two facts: $(A B A)^{s}=A B,(B A B)^{s}=B A$ for some/all of $s \geqslant 1$.

$\langle 121\rangle r\left(M^{2}\right)=r(M)$ and $(A B A)^{s}+(B A B)^{s}=A B+B A$ for some/all of $s \geqslant 1$.

$\langle 122\rangle r\left(M^{2}\right)=r(M)$ and $\left(I_{m}-A B\right)^{s}=I_{m}-A B$ for some/all of $s \geqslant 2$.

$\langle 123\rangle r\left(M^{2}\right)=r(M)$ and $\left(I_{m}-A B\right)^{s}=I_{m}-B A$ for some/all of $s \geqslant 2$.

$\langle 124\rangle r\left(M^{2}\right)=r(M)$ and $\left(I_{m}-A B\right)^{s}=\left(I_{m}-B A\right)^{s}$ for some/all of $s \geqslant 1$.

$\langle 125\rangle r\left(M^{2}\right)=r(M)$ and $(A+B-A B)^{s}=A+B-A B$ for some/all of $s \geqslant 2$.

$\langle 126\rangle r\left(M^{2}\right)=r(M)$ and $(A+B-A B)^{\dagger}=A+B-A B$.

$\langle 127\rangle r\left(M^{2}\right)=r(M)$ and $A+B-A B \in \mathbb{C}_{\mathrm{OP}}^{m}$.

$\langle 128\rangle r\left(M^{2}\right)=r(M)$ and $(A B+B A)^{2 s}=2^{2 s-1}(A B+B A)$ for some/all of $s \geqslant 1$.

$\langle 129\rangle r\left(M^{2}\right)=r(M)$ and $\left(I_{m}-A B-B A\right)^{2}=I_{m}$.

$\langle 130\rangle r\left(M^{2}\right)=r(M)$ with one/both the two facts: $I_{m}+A B \in\left\{\left(I_{m}-A B\right)^{-}\right\}, I_{m}+B A \in\left\{\left(I_{m}-B A\right)^{-}\right\}$.

$\langle 131\rangle r\left(M^{2}\right)=r(M)$ with one/both the two facts: $\left(I_{m}-A B A\right)^{s}=I_{m}-A B A,\left(I_{m}-B A B\right)^{s}=I_{m}-B A B$ for some/all of $s \geqslant 2$.

$\langle 132\rangle r\left(M^{2}\right)=r(M)$ and $\left(I_{m}-A B A\right)^{s}+\left(I_{m}-B A B\right)^{s}=2 I_{m}-A B A-B A B$ for some/all of $s \geqslant 2$.

$\langle 133\rangle r\left(M^{2}\right)=r(M)$ and $(A B A+B A B)^{s}=2^{s-1}(A B A+B A B)$ for some/all of $s \geqslant 2$.

$\langle 134\rangle r\left(M^{2}\right)=r(M)$ and $\left(I_{m}-A B A-B A B\right)^{2}=I_{m}$.

$\langle 135\rangle r\left(M^{2}\right)=r(M)$ with one/both the two facts: $(A-B A B)^{S}=A-B A B,(B-A B A)^{s}=B-A B A$ for some/all of $s \geqslant 2$.

$\langle 136\rangle r\left(M^{2}\right)=r(M)$ and $(A-B A B)^{s}+(B-A B A)^{s}=A+B-A B A-B A B$ for some/all of $s \geqslant 2$.

$\langle 137\rangle r\left(M^{2}\right)=r(M)$ with one/both of the two facts: $A-B A B \succcurlyeq 0, B-A B A \succcurlyeq 0$.

$\langle 138\rangle r\left(M^{2}\right)=r(M)$ and $A+B-A B A-B A B \succcurlyeq 0$.

$\langle 139\rangle r\left(M^{2}\right)=r(M)$ with one/both of the two facts: $A-B A B \in \mathbb{C}_{\mathrm{OP}}^{m}, B-A B A \in \mathbb{C}_{\mathrm{OP}}^{m}$.

$\langle 140\rangle r\left(M^{2}\right)=r(M)$ and $(A+B-A B A-B A B) / 2 \in \mathbb{C}_{\mathrm{OP}}^{m}$.

$\langle 141\rangle r\left(M^{2}\right)=r(M)$ with one/both of the two facts: $A B A+\widetilde{A} B \widetilde{A} \in \mathbb{C}_{\mathrm{OP}}^{m}, B A B+\widetilde{B} A \widetilde{B} \in \mathbb{C}_{\mathrm{OP}}^{m}$.

$\langle 142\rangle r\left(M^{2}\right)=r(M)$ with one/both of the two facts: $A B A+\widetilde{A} B \widetilde{A}=B, B A B+\widetilde{B} A \widetilde{B}=A$.

$\langle 143\rangle r\left(M^{2}\right)=r(M)$ with one/both of the two facts: $A B \widetilde{A}+\widetilde{A} B A=0, B A \widetilde{B}+\widetilde{B} A B=0$.

$\langle 144\rangle r\left(M^{2}\right)=r(M)$ with one/both of the two facts: $(A B)^{\dagger}=B A,(B A)^{\dagger}=A B$.

$\langle 145\rangle r\left(M^{2}\right)=r(M)$ with one/both of the two facts: $(A B)^{\dagger}=A B,(B A)^{\dagger}=B A$.

$\langle 146\rangle r\left(M^{2}\right)=r(M)$ and $(A B)^{\dagger}+(B A)^{\dagger}=A B+B A$.

$\langle 147\rangle r\left(M^{2}\right)=r(M)$ and $\left[(A B)^{2}\right]^{\dagger}=\left[(A B)^{\dagger}\right]^{2}$.

$\langle 148\rangle r\left(M^{2}\right)=r(M)$ and $\left[(A B)^{\dagger}+(B A)^{\dagger}\right] / 2 \in \mathbb{C}_{\mathrm{OP}}^{m}$.

$\langle 149\rangle r\left(M^{2}\right)=r(M)$ with one/both of the two facts: $A B(A B)^{\dagger}=A B A, B A(B A)^{\dagger}=B A B$. 
$\langle 150\rangle r\left(M^{2}\right)=r(M)$ and $A B(A B)^{\dagger}+B A(B A)^{\dagger}=A B A+B A B$.

$\langle 151\rangle r\left(M^{2}\right)=r(M)$ and $(A B)(B A)^{\dagger}=(B A)(A B)^{\dagger}$.

$\langle 152\rangle r\left(M^{2}\right)=r(M)$ and $\left[(A B)(A B)^{\dagger}\right]\left[(B A)(B A)^{\dagger}\right]=\left[(B A)(B A)^{\dagger}\right]\left[(A B)(A B)^{\dagger}\right]$.

$\langle 153\rangle r\left(M^{2}\right)=r(M)$ with one/both of the two facts: $A(\widetilde{A} \widetilde{B})^{\dagger} B=0, B(\widetilde{B} \widetilde{A})^{\dagger} A=0$.

$\langle 154\rangle r\left(M^{2}\right)=r(M)$ and $A(\widetilde{A} \widetilde{B})^{\dagger} B+B(\widetilde{B} \widetilde{A})^{\dagger} A=0$.

$\langle 155\rangle r\left(M^{2}\right)=r(M)$ with one/both of the two facts: $(A B)^{\#} \in \mathbb{C}_{\mathrm{OP}}^{m},(B A)^{\#} \in \mathbb{C}_{\mathrm{OP}}^{m}$.

$\langle 156\rangle r\left(M^{2}\right)=r(M)$ and $\left[(A B)^{\#}+(B A)^{\#}\right] / 2 \in \mathbb{C}_{\mathrm{OP}}^{m}$.

$\langle 157\rangle r\left(M^{2}\right)=r(M)$ with one/both of the two facts: $(A B)^{\#}=(A B)^{\dagger},(B A)^{\#}=(B A)^{\dagger}$.

$\langle 158\rangle r\left(M^{2}\right)=r(M)$ and $(A B)^{\#}+(B A)^{\#}=(A B)^{\dagger}+(B A)^{\dagger}$.

$\langle 159\rangle r\left(M^{2}\right)=r(M)$ with one/both of the two facts: $(A B)(A B)^{\#}=(A B)(A B)^{\dagger},(B A)(B A)^{\#}=(B A)(B A)^{\dagger}$.

$\langle 160\rangle r\left(M^{2}\right)=r(M)$ and $(A B)(A B)^{\#}+(B A)(B A)^{\#}=(A B)(A B)^{\dagger}+(B A)(B A)^{\dagger}$.

$\langle 161\rangle r\left(M^{2}\right)=r(M)$ with one/both of the two facts: $(A B)^{\#}=A B,(B A)^{\#}=B A$.

$\langle 162\rangle r\left(M^{2}\right)=r(M)$ and $(A B)^{\#}+(B A)^{\#}=A B+B A$.

$\langle 163\rangle r\left(M^{2}\right)=r(M)$ and one/both of the two facts: $(A B A)^{\dagger}=A B A,(B A B)^{\dagger}=B A B$.

$\langle 164\rangle r\left(M^{2}\right)=r(M)$ and $(A B A)^{\dagger}+(B A B)^{\dagger}=A B A+B A B$.

$\langle 165\rangle r\left(M^{2}\right)=r(M)$ with one/both of the two facts: $\left(I_{m}-A B\right)^{\dagger}=I_{m}-A B,\left(I_{m}-B A\right)^{\dagger}=I_{m}-B A$.

$\langle 166\rangle r\left(M^{2}\right)=r(M)$ with one/both of the two facts: $\left(I_{m}-A B\right)^{\dagger}=I_{m}-B A,\left(I_{m}-B A\right)^{\dagger}=I_{m}-A B$.

$\langle 167\rangle r\left(M^{2}\right)=r(M)$ and $\left(I_{m}-A B\right)^{\dagger}+\left(I_{m}-B A\right)^{\dagger}=2 I_{m}-A B-B A$.

$\langle 168\rangle r\left(M^{2}\right)=r(M)$ and $\left[\left(I_{m}-A B\right)^{2}\right]^{\dagger}=\left[\left(I_{m}-A B\right)^{\dagger}\right]^{2}$.

$\langle 169\rangle r\left(M^{2}\right)=r(M)$ with one/both of the two facts: $A\left(I_{m}-B A\right)^{\dagger} B=0, B\left(I_{m}-A B\right)^{\dagger} A=0$.

$\langle 170\rangle r\left(M^{2}\right)=r(M)$ and $A\left(I_{m}-B A\right)^{\dagger} B+B\left(I_{m}-A B\right)^{\dagger} A=0$.

$\langle 171\rangle r\left(M^{2}\right)=r(M)$ and $\left[\left(I_{m}-A B\right)^{\dagger}+\left(I_{m}-B A\right)^{\dagger}\right] / 2 \in \mathbb{C}_{\mathrm{OP}}^{m}$.

$\langle 172\rangle r\left(M^{2}\right)=r(M)$ with one/both of the two facts: $\left(2 I_{m}-A-B\right)\left(2 I_{m}-A-B\right)^{\dagger}=I_{m}-A B,\left(2 I_{m}-A-B\right)\left(2 I_{m}-\right.$ $A-B)^{\dagger}=I_{m}-B A$.

$\langle 173\rangle r\left(M^{2}\right)=r(M)$ with one/both of the two facts: $\left(2 I_{m}-A B-B A\right)\left(2 I_{m}-A B-B A\right)^{\dagger}=I_{m}-A B,\left(2 I_{m}-A B-\right.$ $B A)\left(2 I_{m}-A B-B A\right)^{\dagger}=I_{m}-B A$.

$\langle 174\rangle r\left(M^{2}\right)=r(M)$ with one/both of the two facts: $\left(I_{m}-A B A\right)^{\dagger}=I_{m}-A B A,\left(I_{m}-B A B\right)^{\dagger}=I_{m}-B A B$.

$\langle 175\rangle r\left(M^{2}\right)=r(M)$ and $\left(I_{m}-A B A\right)^{\dagger}+\left(I_{m}-B A B\right)^{\dagger}=2 I_{m}-A B A-B A B$.

$\langle 176\rangle r\left(M^{2}\right)=r(M)$ and $\left[(A B A)^{\dagger}+(B A B)^{\dagger}\right] / 2 \in \mathbb{C}_{\mathrm{OP}}^{m}$.

$\langle 177\rangle r\left(M^{2}\right)=r(M)$ with one/both of the two facts: $A\left(I_{m}-A B A\right)^{\dagger} B=0, A\left(I_{m}-B A B\right)^{\dagger} B=0$.

$\langle 178\rangle r\left(M^{2}\right)=r(M)$ and $A\left(I_{m}-A B A\right)^{\dagger} B+A\left(I_{m}-B A B\right)^{\dagger} B=0$.

$\langle 179\rangle r\left(M^{2}\right)=r(M)$ with one/both of the two facts: $\left(2 I_{m}-A B A-B A B\right)\left(2 I_{m}-A B A-B A B\right)^{\dagger}=I_{m}-A B A$,

$\left(2 I_{m}-A B A-B A B\right)\left(2 I_{m}-A B A-B A B\right)^{\dagger}=I_{m}-B A B$.

$\langle 180\rangle r\left(M^{2}\right)=r(M)$ and Trace $\left[(A B)^{s}\right]=\operatorname{Trace}(A B)$ for some/all of $s \geqslant 2$.

$\langle 181\rangle r\left(M^{2}\right)=r(M)$ and $r(A B)=\operatorname{Trace}(A B)$.

$\langle 182\rangle r\left(M^{2}\right)=r(M)$ and Trace $\left[\left(I_{m}-A B\right)^{s}\right]=\operatorname{Trace}\left(I_{m}-A B\right)$ for some/all of $s \geqslant 2$.

$\langle 183\rangle r\left(M^{2}\right)=r(M)$ with one/both of the two facts: Trace[ $\left.\left(I_{m}-A B A\right)^{S}\right]=\operatorname{Trace}\left(I_{m}-A B A\right)$, Trace[ $\left[I_{m}-\right.$

$\left.B A B)^{s}\right]=$ Trace $\left(I_{m}-B A B\right)$ for some/all of $s \geqslant 2$.

$\langle 184\rangle r\left(M^{2}\right)=r(M)$ and $r[A, B]=\operatorname{Trace}(A)+\operatorname{Trace}(B)-\operatorname{Trace}(A B)$.

$\langle 185\rangle r\left(M^{2}\right)=r(M)$ and $r\left(I_{m}-A-B\right)=m-r(A)-r(B)+2 r(A B)$.

$\langle 186\rangle r\left(M^{2}\right)=r(M)$ and $r\left(I_{m}-A-B\right)=m-\operatorname{Trace}(A)-\operatorname{Trace}(B)+2 \operatorname{Trace}(A B)$.

$\langle 187\rangle r\left(M^{2}\right)=r(M)$ and $r(A-B)+r\left(I_{m}-A-B\right)=m$.

$\langle 188\rangle r\left(M^{2}\right)=r(M)$ and $2 r[A, B]=2 r(A)+2 r(B)-r(A B+B A)$.

$\langle 189\rangle r\left(M^{2}\right)=r(M)$ and $r\left(I_{m}-A B\right)=m-r(A B)$.

$\langle 190\rangle r\left(M^{2}\right)=r(M)$ and $r\left(I_{m}-A B\right)=m-\operatorname{Trace}(A B)$.

$\langle 191\rangle r\left(M^{2}\right)=r(M)$ with one $/$ both of the two facts: $\operatorname{Trace}(A)=\operatorname{Trace}(A B)$, $\operatorname{Trace}(B)=\operatorname{Trace}(B A)$.

192〉 $r\left(M^{2}\right)=r(M)$ with one/both of the two facts: $r\left(I_{m}-A B A\right)=m-r(A B A), r\left(I_{m}-B A B\right)=m-r(B A B)$.

$\langle 193\rangle r\left(M^{2}\right)=r(M)$ with one/both of the two facts: $r\left(I_{m}-A B A\right)=m-\operatorname{Trace}(A B A), r\left(I_{m}-B A B\right)=m-$

Trace $(B A B)$.

$\langle 194\rangle r\left(M^{2}\right)=r(M)$ with one/both of the two facts: $B A B=A, A B A=B$.

$\langle 195\rangle r\left(M^{2}\right)=r(M)$ with one/both of the two facts: $A=P_{\mathscr{R}(M) \cap \mathscr{R}\left(M^{*}\right)}$ and $B=P_{\mathscr{R}(M) \cap \mathscr{R}\left(M^{*}\right)}$. 
$\langle 196\rangle r\left(M^{2}\right)=r(M)$ and $A+B=2 P_{\mathscr{R}(M) \cap \mathscr{R}\left(M^{*}\right)}$.

$\langle 197\rangle r\left(M^{2}\right)=r(M)$ and $I_{m}-A-B=-P_{\mathscr{R}(M) \cap \mathscr{R}\left(M^{*}\right)}+P_{\mathscr{R}}^{\perp}(M) \cap \mathscr{R} \perp\left(M^{*}\right)$.

$\langle 198\rangle r\left(M^{2}\right)=r(M)$ and $\mathscr{R}\left(I_{m}-A-B\right)=\left(\mathscr{R}(M) \cap \mathscr{R}\left(M^{*}\right)\right) \oplus\left(\mathscr{R}^{\perp}(M) \cap \mathscr{R}^{\perp}\left(M^{*}\right)\right)$.

$\langle 199\rangle r\left(M^{2}\right)=r(M)$ and $P_{\mathscr{R}\left(I_{m}-A-B\right)}=P_{\mathscr{R}(M) \cap \mathscr{R}\left(M^{*}\right)}+P_{\mathscr{R} \perp}(M) \cap \mathscr{R} \perp\left(M^{*}\right) \cdot$

$\langle 200\rangle r\left(M^{2}\right)=r(M)$ and $\mathscr{R}(A-B) \oplus \mathscr{R}\left(I_{m}-A-B\right)=\mathbb{C}^{m}$.

$\langle 201\rangle r\left(M^{2}\right)=r(M)$ with one/both of the two facts: $A B=P_{\mathscr{R}(M) \cap \mathscr{R}\left(M^{*}\right)}, B A=P_{\mathscr{R}(M) \cap \mathscr{R}\left(M^{*}\right)}$.

$\langle 202\rangle r\left(M^{2}\right)=r(M)$ and $A B+B A=2 P_{\mathscr{R}(M) \cap \mathscr{R}\left(M^{*}\right)}$.

$\langle 203\rangle r\left(M^{2}\right)=r(M)$ with one/both of the two facts: $\mathscr{R}(A B)=\mathscr{R}(M) \cap \mathscr{R}\left(M^{*}\right), \mathscr{R}(B A)=\mathscr{R}(M) \cap \mathscr{R}\left(M^{*}\right)$.

$\langle 204\rangle r\left(M^{2}\right)=r(M)$ with one/both of the two facts: $A B=P_{\mathscr{R}(A B)}, B A=P_{\mathscr{R}(B A)}$.

$\langle 205\rangle r\left(M^{2}\right)=r(M)$ and $A B+B A=P_{\mathscr{R}(A B)}+P_{\mathscr{R}(B A)}$.

$\langle 206\rangle r\left(M^{2}\right)=r(M)$ and $\mathscr{R}\left(I_{m}-A B\right)=\mathscr{R}\left(I_{m}-B A\right)=\mathscr{R}^{\perp}(M) \cap \mathscr{R}^{\perp}\left(M^{*}\right)$.

$\langle 207\rangle r\left(M^{2}\right)=r(M)$ and $I_{m}-A B=P_{\mathscr{R}} \perp(M) \cap \mathscr{R} \perp\left(M^{*}\right)$.

$\langle 208\rangle r\left(M^{2}\right)=r(M)$ with one/both of the two facts: $\mathscr{R}(A B) \cap \mathscr{R}\left(I_{m}-A B\right)=\{0\}, \mathscr{R}(B A) \cap \mathscr{R}\left(I_{m}-B A\right)=\{0\}$.

$\langle 209\rangle r\left(M^{2}\right)=r(M)$ with one/both of the two facts: $\mathbb{C}^{m}=\mathscr{R}(A B) \oplus \mathscr{R}\left(I_{m}-A B\right), \mathbb{C}^{m}=\mathscr{R}(B A) \oplus \mathscr{R}\left(I_{m}-B A\right)$.

$\langle 210\rangle r\left(M^{2}\right)=r(M)$ with one/both of the two facts: $I_{m}=P_{\mathscr{R}(A B)}+P_{\mathscr{R}\left(I_{m}-A B\right)}, I_{m}=P_{\mathscr{R}(B A)}+P_{\mathscr{R}\left(I_{m}-B A\right)}$.

$\langle 211\rangle r\left(M^{2}\right)=r(M)$ with one/both of the two facts: $A B A=P_{\mathscr{R}(M) \cap \mathscr{R}\left(M^{*}\right)}, B A B=P_{\mathscr{R}(M) \cap \mathscr{R}\left(M^{*}\right)}$.

$\langle 212\rangle r\left(M^{2}\right)=r(M)$ and $A B A+B A B=2 P_{\mathscr{R}(M) \cap \mathscr{R}\left(M^{*}\right)}$.

$\langle 213\rangle r\left(M^{2}\right)=r(M)$ and $\mathscr{R}(A B+B A)=\mathscr{R}(M) \cap \mathscr{R}\left(M^{*}\right)$.

$\langle 214\rangle r\left(M^{2}\right)=r(M)$ and $\mathscr{R}(A B)=\mathscr{R}(B A)$.

$\langle 215\rangle r\left(M^{2}\right)=r(M)$ with one/both of the two facts: $\mathscr{R}(A B) \oplus \mathscr{N}(A B)=\mathbb{C}^{m}, \mathscr{R}(B A) \oplus \mathscr{N}(B A)=\mathbb{C}^{m}$.

$\langle 216\rangle r\left(M^{2}\right)=r(M)$ with one/both of the two facts: $\mathscr{R}(A B)=\mathscr{R}(A) \cap \mathscr{R}(B), \mathscr{R}(B A)=\mathscr{R}(A) \cap \mathscr{R}(B)$.

$\langle 217\rangle r\left(M^{2}\right)=r(M)$ with one/both of the two facts: $\mathscr{R}(\widetilde{A} \widetilde{B})=\mathscr{N}(A) \cap \mathscr{N}(B), \mathscr{R}(\widetilde{B} \widetilde{A})=\mathscr{N}(A) \cap \mathscr{N}(B)$.

$\langle 218\rangle r\left(M^{2}\right)=r(M)$ and $\mathscr{R}(A B \widetilde{A})=\mathscr{R}(B A \widetilde{B})$.

Acknowledgements. The author would like to express his thanks to the referee for helpful comments and suggestions to an earlier version of this paper.

\section{References}

[1] S. Afriat. On the latent vectors and characteristic values of products of pairs of symmetric idempotents. Quart. J. Math. 7(1956), 76-78.

[2] S. Afriat. Orthogonal and oblique projectors and the characteristics of pairs of vector spaces. Proc. Cambridge Phil. Soc. 53(1957), 800-816.

[3] W.O. Amrein, K.B. Sinha. On pairs of projections in a Hilbert space. Linear Algebra Appl. 208/209(1994), 425-435.

[4] E. Andruchow, G. Corach. Essentially orthogonal subspaces. J. Oper. Theory 79(2018), 79-100.

[5] A. Arias, S. Gudder. Almost sharp quantum effects. J. Math. Phys. 45(2004), 4196-4206.

[6] J.E. Avron, R. Seiler, B. Simon. The index of a pair of projections. J. Func. Anal. 120(1994), 220-237.

[7] J.K. Baksalary. Algebraic characterizations and statistical implications of the commutativity of orthogonal projectors. In: Proceedings of the Second International Tampere Conference in Statistics (T. Pukkila and S. Puntanen eds.), University of Tampere, Tampere, Finland, 1987, pp. 113-142.

[8] J.K. Baksalary, O.M. Baksalary. Commutativity of projectors. Linear Algebra Appl. 341(2002), 129-142.

[9] J.K. Baksalary, O.M. Baksalary, P. Kik. Generalizations of a property of orthogonal projectors. Linear Algebra Appl. 420(2007), 1-8.

[10] J.K. Baksalary, O.M. Baksalary, T. Szulc. A property of orthogonal projectors. Linear Algebra Appl. 354(2002), 35-39.

[11] J.K. Baksalary, G.P.H. Styan. Around a formula for the rank of a matrix product with some statistical applications. In Graphs, Matrices, and Designs: Festschrift in Honor of N.J. Pullman on his Sixtieth Birthday (R.S. Rees ed.), Marcel Dekker, New York, 1993, pp. 1-18.

[12] O.M. Baksalary, P. Kik. On commutativity of projectors. Linear Algebra Appl. 417(2006), 31-41.

[13] O.M. Baksalary, G. Trenkler. Characterizations of EP, normal, Hermitian matrices. Linear Multilinear Algebra 56(2008), 299304.

[14] O.M. Baksalary, G. Trenkler. An alternative approach to characterize the commutativity of orthogonal projectors. Discuss. Math. Probab. Stat. 28(2008), 113-137.

[15] O.M. Baksalary, G. Trenkler. Column space equalities for orthogonal projectors. Appl. Math. Comput. 212(2009), 519-529. 
[16] O.M. Baksalary, G. Trenkler. Revisitation of the product of two orthogonal projectors. Linear Algebra Appl. 430(2009), 28132833.

[17] O.M. Baksalary, G. Trenkler. Eigenvalues of functions of orthogonal projectors. Linear Algebra Appl. 431(2009), $2172-2186$.

[18] O.M. Baksalary, G. Trenkler. On angles and distances between subspaces. Linear Algebra Appl. 431(2009), 2243-2260.

[19] O.M. Baksalary, G. Trenkler. On a subspace metric based on matrix rank. Linear Algebra Appl. 432(2010), 1475-1491.

[20] O.M. Baksalary, G. Trenkler. Functions of orthogonal projectors involving the Moore-Penrose inverse. Comput. Math. Appl. 59(2010), 764-778.

[21] O.M. Baksalary, G. Trenkler. On the projectors $F F^{\dagger}$ and $F^{\dagger} F$. Appl. Math. Comput. 217(2011), 10213-10223.

[22] O.M. Baksalary, G. Trenkler. Rank formulae from the perspective of orthogonal projectors. Linear Multilinear Algebra 59(2011), 607-625.

[23] O.M. Baksalary, G. Trenkler. On disjoint range matrices. Linear Algebra Appl. 435(2011), 1222-1240.

[24] O.M. Baksalary, G. Trenkler. On the entries of orthogonal projection matrices. In: Combinatorial Matrix Theory and Generalized Inverses of Matrices, R. Bapat, S. Kirkland, K. Prasad, S. Puntanen (eds), Springer, India, 2013, pp. 101-118.

[25] H. Behncke. Projections in Hilbert Space. Tahôku Math. J. 22(1970), 181-183.

[26] H. Behncke. Projections in Hilbert Space II. Tahôku Math. J. 23(1971), 349-352.

[27] J. Benítez, X. Liu. A short proof of a matrix decomposition with applications. Linear Algebra Appl. 438(2013), $1398-1414$.

[28] J. Benítez, V. Rakocěvić. Applications of CS decomposition in linear combinations of two orthogonal projectors. Appl. Math. Comput. 203(2008), 761-769.

[29] J. Benítez, V. Rakocěvić. On the spectrum of linear combinations of two projections in $C^{*}$-algebras. Linear Multilinear Algebra 58(2010), 673-679.

[30] J. Benítez, V. Rakocěvić. Matrices $A$ such that $A A^{\dagger}-A^{\dagger} A$ are nonsingular. Appl. Math. Comput. 217(2010), 3493-3503.

[31] A. Ben-Israel. On the geometry of subspaces in Euclidean $n$-spaces. SIAM J. Appl. Math. 15(1967), 1184-1198.

[32] A. Ben-Israel, T.N.E. Greville. Generalized Inverses: Theory and Applications. 2nd ed., Springer, New York, 2003.

[33] A.M. Bikchentaev. On representation of elements of a von Neumann algebra in the form of finite sums of products of projections. Siber. Math. J. 46(2005), 24-34.

[34] A.M. Bikchentaev. Commutativity of projectors and trace characterization on von Neumann algebras. I. Russian Math. 53(2009), 68-71.

[35] A.M. Bikchentaev. Commutation of projections and trace characterization on von Neumann algebras. II. Math. Notes 89(2011), 461-471.

[36] A.M. Bikchentaev. Commutation of projections and characterization of traces on von Neumann algebras. III. Int. J. Theor. Phys. 54(2015), 4482-4493.

[37] A. Björck, G. Golub. Numerical methods for computing angles between linear subspaces. Math. Comput. 27(1973), 579-594.

[38] P.E. Bjørstad, J. Mandel. On the spectra of sums of orthogonal projections with applications to parallel computing. BIT 31(1991), 76-88.

[39] E. Boasso. On the Moore-Penrose inverse, EP Banach space operators, and EP Banach algebra elements. J. Math. Anal. Appl. 339(2008), 1003-1014.

[40] E. Boasso. Factorizations of EP Banach space operators and EP Banach algebra elements. J. Math. Anal. Appl. 379(2011), 245-255.

[41] S. Borac. On the algebra generated by two projections. J. Math. Phys. 36(1995), 863-874.

[42] A. Böttcher, I.M. Spitkovsky. Drazin inversion in the von Neumann algebra generated by two orthogonal projections. J. Math. Anal. Appl. 358(2009), 403-409.

[43] A. Böttcher, I.M. Spitkovsky. A gentle guide to the basics of two projections theory. Linear Algebra Appl. 432(2010), 14121459.

[44] A. Böttcher, I.M. Spitkovsky. On certain finite-dimensional algebras generated by two idempotents. Linear Algebra Appl. 435(2011), 1823-1836.

[45] A. Böttcher, I.M. Spitkovsky. Group inversion in certain finite-dimensional algebras generated by two idempotents. Indaga. Math. 23(2012), 715-732.

[46] A. Böttcher, I.M. Spitkovsky.Classification of the finite-dimensional algebras generated by two tightly coupled idempotents. Linear Algebra Appl. 439(2013), 538-551.

[47] S. Brenner. Endomorphism algebras of vector spaces with distinguished sets of subspaces. J. Algebra 6(1967), $100-114$.

[48] S.L. Campbell, C.D. Meyer. Generalized Inverses of Linear Transformations. Corrected reprint of the 1979 original, Dover, New York, 1991.

[49] S. Cheng, Y. Tian. Moore-Penrose inverses of products and differences of orthogonal projectors. Acta Sci. Math. (Szeged) 69(2003), 533-542.

[50] M.-D. Choi, P.Y. Wu. Convex combinations of projections. Linear Algebra Appl. 136(1990), 25-42.

[51] M.-D. Choi, P.Y. Wu. Sums of orthogonal projections. J. Funct. Anal. 267(2014), 384-404.

[52] G. Corach, A. Maestripieri. Products of orthogonal projections and polar decompositions. Linear Algebra Appl. 434(2011), 1594-1609.

[53] J. Dauxois, G.M. Nkiet. Canonical analysis of two Euclidean subspaces and its applications. Linear Algebra Appl. 264(1997), 355-388. 
[54] C. Davis. Separation of two linear subspaces. Acta Sci. Math. (Szeged) 19(1958), 172-187.

[55] E.R. De Arellano, N.L. Vasilevsky. Algebras of singular integeral operators generated by three othogonal projections. Integr. Equ. Oper. Theory 25(1996), 277-288.

[56] F. Deutsch. The angle between subspaces of a Hilbert space. In: Approximation Theory, Wavelets and Applications, S. Singh (ed.), Kluwer, Dordrecht, 1995, pp. 107-130.

[57] J. Dixmier. Position relative de deux variétés linéaires fermées dans un espace de Hilbert. Rev. Sci. 86(1948), 387-399.

[58] D.S. Djordjević. Product of EP operators on Hilbert spaces. Proc. Amer. Math. Soc. 129(2000), 1727-1731.

[59] D.S. Djordjević. Characterizations of normal, hyponormal and EP operators. J. Math. Anal. Appl. 329(2007), 1181-1190.

[60] D.S. Djordjević, J.J. Koliha. Characterizing hermitian, normal and EP operators. Filomat 21(2007), 39-54.

[61] D. Djordjević, J.J. Koliha, I. Straškraba. Factorization of EP elements in $C^{*}$-algebras. Linear Multilinear Algebra 57(2009), 587-594.

[62] D. Drivaliaris, S. Karanasios, D. Pappas. Factorizations of EP operators. Linear Algebra Appl. 429(2008), 1555-1567.

[63] Y.-N. Dou, Y.-Q. Wang, M.-M. Cui. H.-K. Du. Spectra of anticommutator for two orthogonal projections. Linear Multilinear Algebra 67(2019), 2077-2081.

[64] H.-K. Du, C.-Y. Deng. Moore-Penrose inverses of products and differences of orthogonal projections. Acta Anal. Funct. Appl. 8(2006), 104-109.

[65] H.-K. Du, G. Gao, Y. Wang. Geometry characterizations between two subspaces (in Chinese). Acta Math. Scientia 32(2012), 879-891.

[66] M. Enomoto, Y. Watatani. Relative position of four subspaces in a Hilbert space. Adv. Math. 201(2006), 263-317.

[67] J. Farokhi-Ostad, A.R. Janfada. Products of EP operators on Hilbert $C^{*}$-modules. Sahand Commun. Math. Anal. 10(2018), 61-71.

[68] I.S. Feshchenko. On closeness of the sum of $n$ subspaces of a Hilbert space. Ukrainian Math. J. 63(2012), $1566-1622$.

[69] P.A. Fillmore. On sums of projections. J. Funct. Anal. 4(1969), 146-152.

[70] I.M. Gelfand, V.A. Ponomarev. Problems of linear algebra and classification of quadruples of subspaces in a finitedimensional vector space. Coll. Math. Spc. Bolyai 5(1970), 163-237.

[71] A. Galántai. Subspaces, angles and pairs of orthogonal projections. Linear Multilinear Algebra 56(2008), $227-260$.

[72] A. Galántai, Cs.J. Hegedüs. Jordan's principal angles in complex vector spaces. Numer. Linear Algebra Appl. 13(2006), 589598.

[73] R. Giles, H. Kummer. A matrix representation of a pair of projections in a Hilbert space. Canad. Math. Bull. 14(1971), 35-44.

[74] G.H. Golub, C.F. Van Loan. Matrix Computations. 3rd edition, Baltimore, Johns Hopkins University Press, 1996.

[75] T.N.E. Greville. Solutions of the matrix equations $X A X=X$ and relations between oblique and orthogonal projectors. SIAM J. Appl. Math. 26(1974), 828-832.

[76] J. Groß. On the product of orthogonal projectors. Linear Algebra Appl. 289(1999), 141-150.

[77] J. Groß. On oblique projection, rank additivity and the Moore-Penrose inverse of the sum of two matrices. Linear Multilinear Algebra 46(1999), 265-275.

[78] S. Gudder. Sharp and unsharp quantum effects. Adv. Appl. Math. 20(1998), 169-187.

[79] P.R. Halmos. Two subspaces. Trans. Amer. Math. Soc. 144(1969), 381-389.

[80] P.R. Halmos. Finite-dimensional Hilbert spaces. Amer. Math. Month. 77(1970), 457-464.

[81] P.R. Halmos. Finite-dimensional Vector Spaces. Springer, New York, 1974.

[82] P.R. Halmos. A Hilbert Space Problem Book. 2nd Ed., Springer, New York 1982.

[83] I. Halperin. The product of projection operators. Acta Sci. Math. (Szeged) 23(1962), 96-99.

[84] R.E. Hartwig, I.J. Katz. On products of EP matrices. Linear Algebra Appl. 252(1997), 339-345.

[85] R.E. Hartwig, K. Spindelböck. Matrices for which $A^{*}$ and $A^{\dagger}$ can commute. Linear Multilinear Algebra 14(1984), $241-256$.

[86] M. Hegland, J. Garcke, V. Challis. The combination technique and some generalisations. Linear Algebra Appl. 420(2007), 249-275.

[87] Kh.D. Ikramov. A canonical form for projectors under unitary similarity. Comput. Math. Math. Phys. 36(1996), $279-281$.

[88] M. Itoh. On some EP operators. Nihonkai Math. J. 16(2005), 49-56.

[89] B. Jiang, Y. Tian. Necessary and sufficient conditions for nonlinear matrix identities to always hold. Aequat. Math. 93(2019), 587-600.

[90] J.J. Koliha. A simple proof of the product theorem for EP matrices. Linear Algebra Appl. 294(1999), 213-215.

[91] J.J. Koliha. Elements of $C^{*}$-algebras commuting with their Moore-Penrose inverse. Studia Math. 139(2000), 81-90.

[92] J.J. Koliha, V. Rakocěvić. Fredholm properties of the difference of orthogonal projections in a Hilbert space. Integr. Equ. Oper. Theory 52(2005), 125-134.

[93] T. Kollo, D. von Rosen. Advanced Multivariate Statistics with Matrices. Springer, 2005.

[94] S.A. Kruglyak, V.I. Rabanovich, Yu.S. Samoilenko. On sums of projections. Funct. Anal. Appl. 36(2002), 182-195.

[95] S.A. Kruglyak, V.I. Rabanovich, Yu.S. Samoilenko. Decomposition of a scalar matrix into a sum of orthogonal projections. Linear Algebra Appl. 370(2003), 217-225.

[96] S.A. Kruglyak, Yu.S. Samoilenko. Structural theorems for families of idempotents. Ukrain. Math. J. 50(1998), 593-604.

[97] A.A. Kyrychenko, Yu.S. Samoilenko. On the spectrum and spectrum multiplicities of a sum of orthogonal projections. Algebra Disc. Math. 3(2004), 71-76. 
[98] A. Lenard. The numerical range of a pair of projections. J. Funct. Anal. 10(1972), 410-423.

[99] Y. Li. The Moore-Penrose inverses of products and differences of projections in a $C^{*}$-algebra. Linear Algebra Appl. 428(2008), 1169-1177.

[100] W.E. Longstaff, O. Panaia. On complementary subspaces of Hilbert space. Proc. Amer. Math. Soc. 126(1988), 3019-3026.

[101] W. López. A new way of computing the orthogonal projection onto the intersection of two hyperplanes in a finitedimensional Hilbert space. Appl. Math. E-Notes 18(2018), 116-123.

[102] L.W. Marcoux. Projections, commutators and Lie ideals in $C^{*}$-algebras. Math. Proc. R. Ir. Acad. 110A(2010), 31-55.

[103] G. Marsaglia, G.P.H. Styan. Equalities and inequalities for ranks of matrices. Linear Multilinear Algebra 2(1974), $269-292$.

[104] N. Matzakos, D. Pappas. EP matrices: computation of the Moore-Penrose inverse via factorizations. J. Appl. Math. Comput. 34(2010), 113-127.

[105] C.D. Meyer. Matrix Analysis and Applied Linear Algebra. SIAM, Philadelphia, 2000.

[106] J. Miao, A. Ben-Israel. On principal angles between subspaces in $\mathbb{R}^{n}$. Linear Algebra Appl. 171(1992), 81-98.

[107] J. Miao, A. Ben-Israel. Product cosines of angles between subspaces. Linear Algebra Appl. 237/238(1996), 71-81.

[108] Yu.P. Moskaleva, Yu.S. Samoilenko. Systems of $n$ subspaces and representations of $*$-algebras generated by projections. Methods Funct. Anal. Topology 12(2006), 57-73.

[109] H. Nakano. Spectral Theory in the Hilbert Space. Japan Society for the Promotion of Science, Tokyo, 1953.

[110] M. Nees. Products of orthogonal projections as Carleman operators. Integr. Equ. Oper. Theory 35(1999), 85-92.

[111] K. Nishio. The structure of a real linear combination of two projections. Linear Algebra Appl. 66(1985), 169-176.

[112] K. Nordström, D. von Rosen. Algebra of subspaces with applications to problems in statistics. In: Proceedings of the Second International Tampere Conference in Statistics (T. Pukkila and S. Puntanen eds.), University of Tampere, Tampere, Finland, 1987, pp. 603-614.

[113] T. Oikhberg. Products of orthogonal Projections. Proc. Amer. Math. Soc. 127(1999), 3659-3669.

[114] C.C. Paige, M. Wei. History and generality of the CS decomposition. Linear Algebra Appl. 1994, 208/209(1994), 303-326.

[115] P.V. Pethe, N.K. Thakare. Convex sums of orthogonal projections on a Banach space. Math. Student 45(1977), 49-52.

[116] R. Piziak, P.L. Odell, R. Hahn. Constructing projections on sums and intersections. Comput. Math. Appl. 37(1999), 67-74.

[117] S. Puntanen, G.P.H. Styan, J. Isotalo. Matrix Tricks for Linear Statistical Models: Our Personal Top Twenty. Springer, 2011.

[118] I. Raeburn, A.M. Sinclair. The $C^{*}$-algebra generated by projections. Math. Scandinavica 65(1989), 278-290.

[119] V. Rakocěvić, H.K. Wimmer. A variational characterization of canonical angles between subspaces. J. Geometry 78(2003), 122-124.

[120] C.R. Rao, S.K. Mitra. Generalized Inverse of Matrices and Its Applications. Wiley, New York, 1971.

[121] W. Rehder. Spectral properties of products of projections in quantum probability theory. Internat. J. Theor. Phys. 18(1979), 791-805.

[122] W. Rehder. On the commutativity of two projections. Elem. Math. 35(1980), 120-122.

[123] W. Rehder. When do projections commute? Z. Naturforsch. 35A(1980), 437-441.

[124] I.B. Risteski, K.G. Trencevski. Principal values and principal subspaces of two subspaces of vector spaces with inner product. Beitr. Algebra Geom. 42(2001), 289-300.

[125] A.G. Robertson. Linear and convex combinations of projections in simple $C^{*}$-algebras. Proc. Roy. Soc. Edinburgh Sect. A 101(1985), 125-129.

[126] S. Roman. Advanced Linear Algebra. Springer, New York, 1992.

[127] H. Schwerdtfeger. Introduction to Linear Algebra and the Theory of Matrices. Noordhoff, Groningen, 1950.

[128] K. Sharifi. EP modular operators and their products. J. Math. Anal. Appl. 419(2014), 870-877.

[129] W. Shi, G. Ji, H.-K. Du. Pairs of orthogonal projections with a fixed difference. Linear Algebra Appl. 489(2016), $288-297$.

[130] G.W. Stewart. Computing the CS decomposition of a partitioned orthonormal matrix. Numer. Math. 40(1982), 297-306.

[131] I.M. Spitkovsky. Once more on algebras generated by two projections. Linear Algebra Appl. 208/209(1994), 377-395.

[132] I.M. Spitkovsky. On polynomials in two projections. Electron. J. Linear Algebra 15(2006), 154-158.

[133] Y. Tian. Upper and lower bounds for ranks of matrix expressions using generalized inverses. Linear Algebra Appl. 355(2002), 187-214.

[134] Y. Tian. The dimension of the intersection of $k$ subspaces. Missouri J. Math. Sci. 14(2002), 1-4.

[135] Y. Tian. Using rank formulas to characterize equalities for Moore-Penrose inverses of matrix products. Appl. Math. Comput. 147(2004), 581-600.

[136] Y. Tian. Equalities for orthogonal projectors and their operations. Centr. Eur. J. Math. 8(2010), 855-870.

[137] Y. Tian. Eight expressions for generalized inverses of a bordered matrix. Linear Multilinear Algebra 58(2010), $203-220$.

[138] Y. Tian. Expansion formulas for the inertias of Hermitian matrix polynomials and matrix pencils of orthogonal projectors. J. Math. Anal. Appl. 376(2011), 162-186.

[139] Y. Tian. Formulas for calculating the dimensions of the sums and the intersections of a family of linear subspaces with applications. Beitr. Algebra Geom. 60(2019), 471-485.

[140] Y. Tian. Linear statistical models, least-squares estimators, and classification analysis to reverse-order laws for generalized inverses of matrix products. doi:10.20944/preprints201812.0342.v1.

[141] Y. Tian, Y. Li. Distributions of eigenvalues and inertias of some block Hermitian matrices consisting of orthogonal projectors. Linear Multilinear Algebra 60(2012), 1027-1069. 
[142] Y. Tian, G.P.H. Styan. Rank equalities for idempotent and involutory matrices. Linear Algebra Appl. 335(2001), 101-117.

[143] Y. Tian, G.P.H. Styan. Rank equalities for idempotent matrices with applications. J. Comput. Appl. Math. 191(2006), 77-97.

[144] Y. Tian, H. Wang. Characterizations of EP matrices and weighted-EP matrices. Linear Algebra Appl. 34(2011), $1295-1318$.

[145] Y. Tian, Y. Wang. Expansion formulas for orthogonal projectors onto ranges of row block matrices. J. Math. Res. Appl. 34(2014), 147-154.

[146] G. Trenkler. Characterizations of oblique and orthogonal projectors. In: Proceedings of the International Conference on Linear Statistical Inference LINSTAT '93, T. Cali/'nski, R. Kala (eds.), Mathematics and Its Applications, vol 306. Springer, Dordrecht 1994, pp. 255-270.

[147] G. Trenkler. Problem 31-7: On the product of orthogonal projectors. IMAGE, Bull. Internat. Linear Algebra Soc. 31(2003), 43.

[148] N.L. Vasilevsky. $C^{*}$-algebras generated by othogonal projections and their applications. Integr. Equ. Oper. Theory 31(1998), $113-132$.

[149] N.L. Vasilevsky, I.M. Spitkovsky. On the algebra generated by two projections (in Russian). Dokl. Akad. Nauk Ukrain. SSR Ser. A 8(1981), 10-13.

[150] P. Wedin. On angles between subspaces of a finite dimensional inner product space. In: Matrix Pencils, Lecture Notes Math. 973(1983), 263-285.

[151] H.K. Wimmer. Canonical angles of unitary spaces and perturbations of direct complements. Linear Algebra Appl. 287(1999), 373-379.

[152] N. Zhang, Y. Wei. Solving EP singular linear systems. Internat. J. Comput. Math. 81(2004), 1395-1405.

[153] X. Zhang, S. Zhang, J. Chen, L. Wang. Moore-Penrose invertibility of differences and products of projections in rings with involution. Linear Algebra Appl. 439(2013), 4101-4109.

[154] K. Zuo, T. Xie. Nonsingularity of the combinations of two orthogonal projectors. Wuhan Univ. J. Nat. Sci. 17(2012), 7-11. 